\title{
Cycloparaphenylene Lemniscates and Trefoils
}

Steven M. Bachrach*

School of Science, Monmouth University, 400 Cedar Avenue, West Long Branch, NJ 07764

sbachrac@monmouth.edu

Manuscript ID: jo-2019-02742e

\section{Supporting Materials}

Group equivalent reactions used to compute ring strain energies, additional tables, and figures, and coordinates of all structures.

Table of Contents

Scheme S1. Group equivalent reactions to determine RSE of 1-10.

Figure S1. Overlay of the geometry of 1-8 computed at BP86-D3/6-311G(d) and $\omega B 97 X-D / 6-311 G(d)$.

Figure S2. Comparison of the RSE of 1-4 - 1-8 computed at BP86-D3/6-311G(d) and $\omega B 97 X-D / 6-311 G(d)$.

Figure S3. Projection of the heavy atom coordinates of 2-4 (green), 2-5 (yellow), 2-6

(light blue), 2-7 (red) and 2-8 (dark blue) along with best fit Booth lemniscates curves.

Figure S4. Dihedral rotational energy curves for H-K. $\quad$ S6

$\begin{array}{ll}\text { Figure S5. Bending }(\phi) \text { energy curves for H-K. } & \text { S7 }\end{array}$

Table S1. Ring Strain Energy ( $\left.\mathrm{kcal} \mathrm{mol}^{-1}\right)$ for 5-10. S8

Optimized geometries and enthalpies at $\omega B 97 X-D / 6-311 \mathrm{~g}(\mathrm{~d}) \quad$ S9 
Scheme S1. Group equivalent reactions to determine RSE of 1-10.
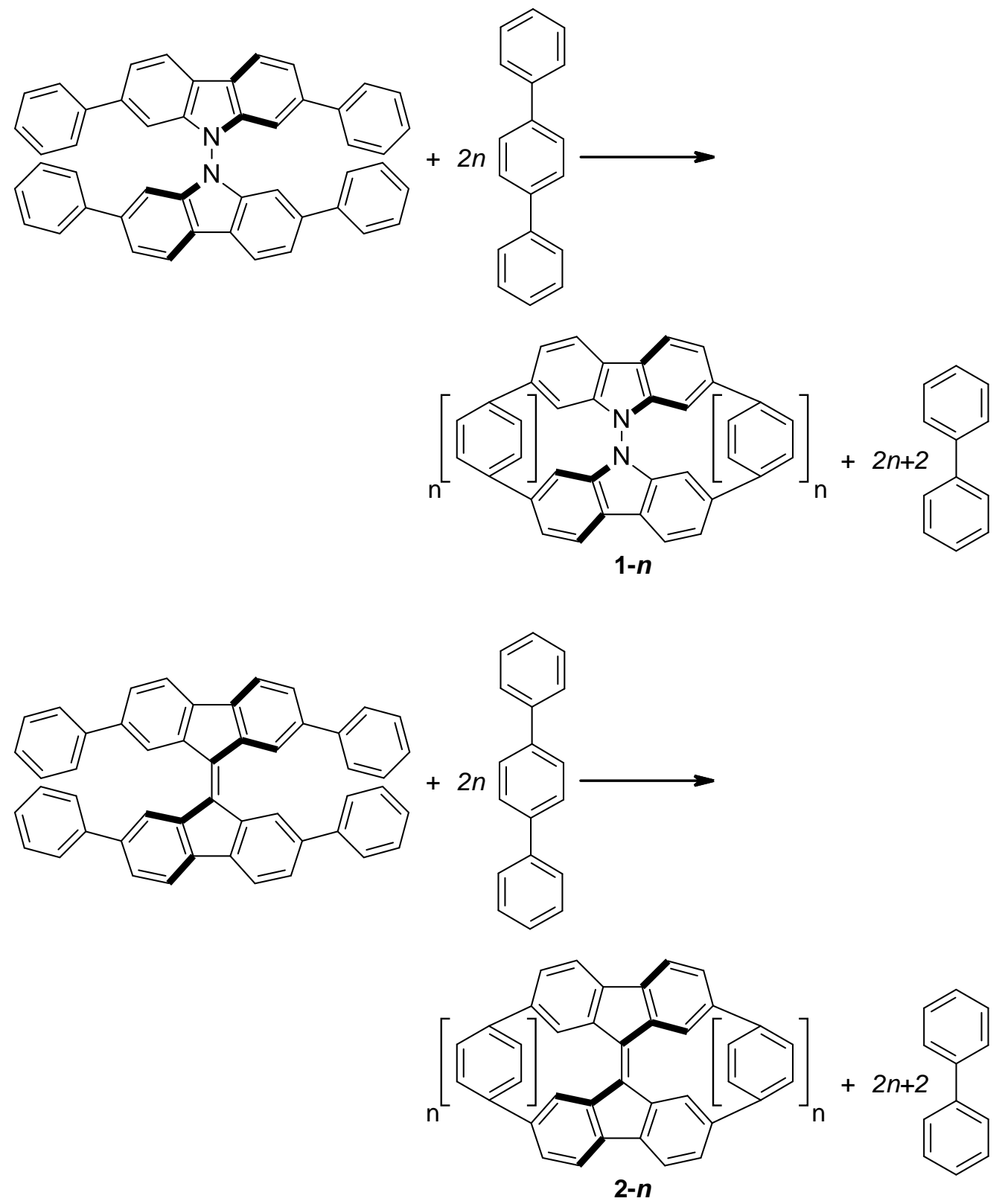

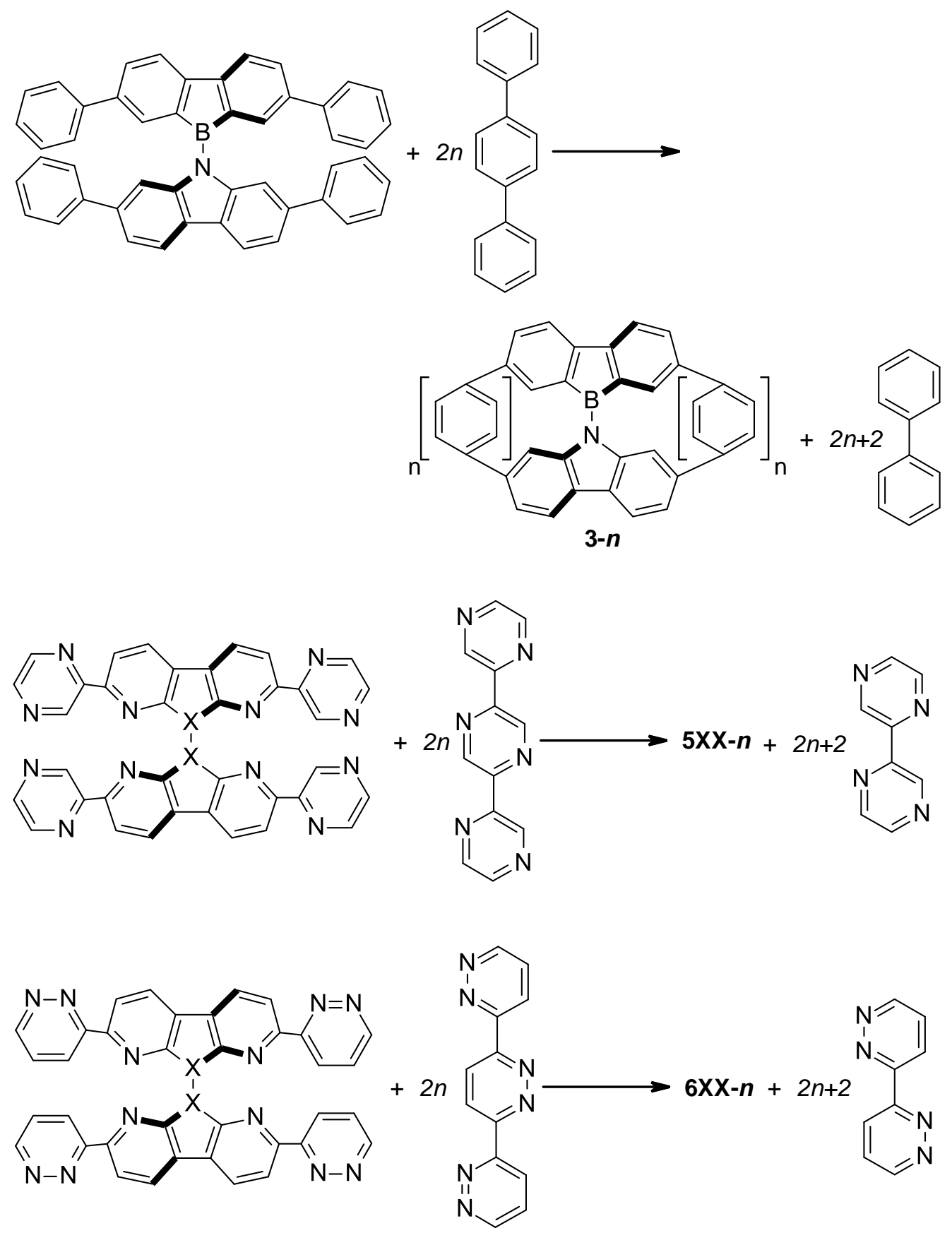

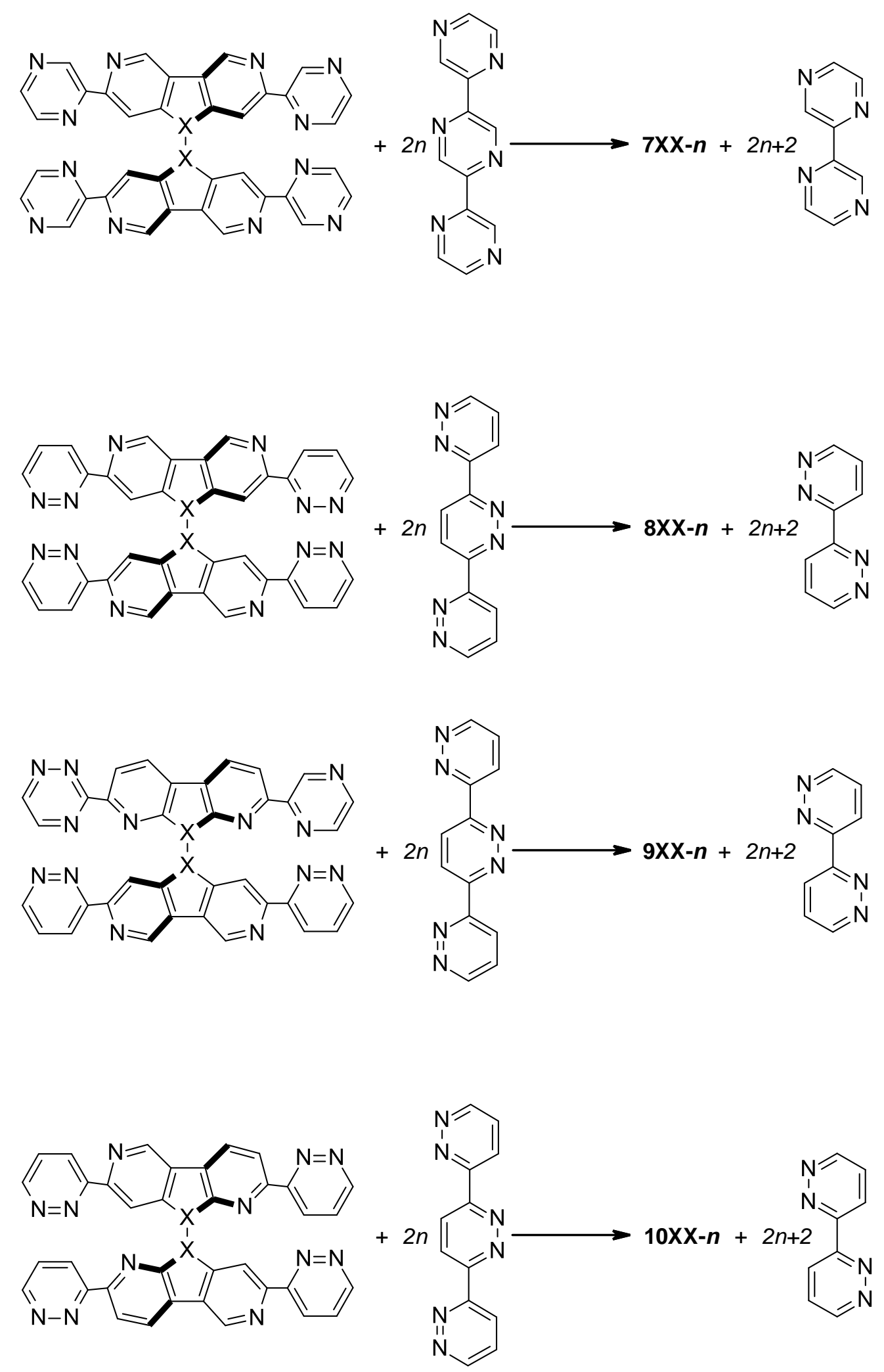


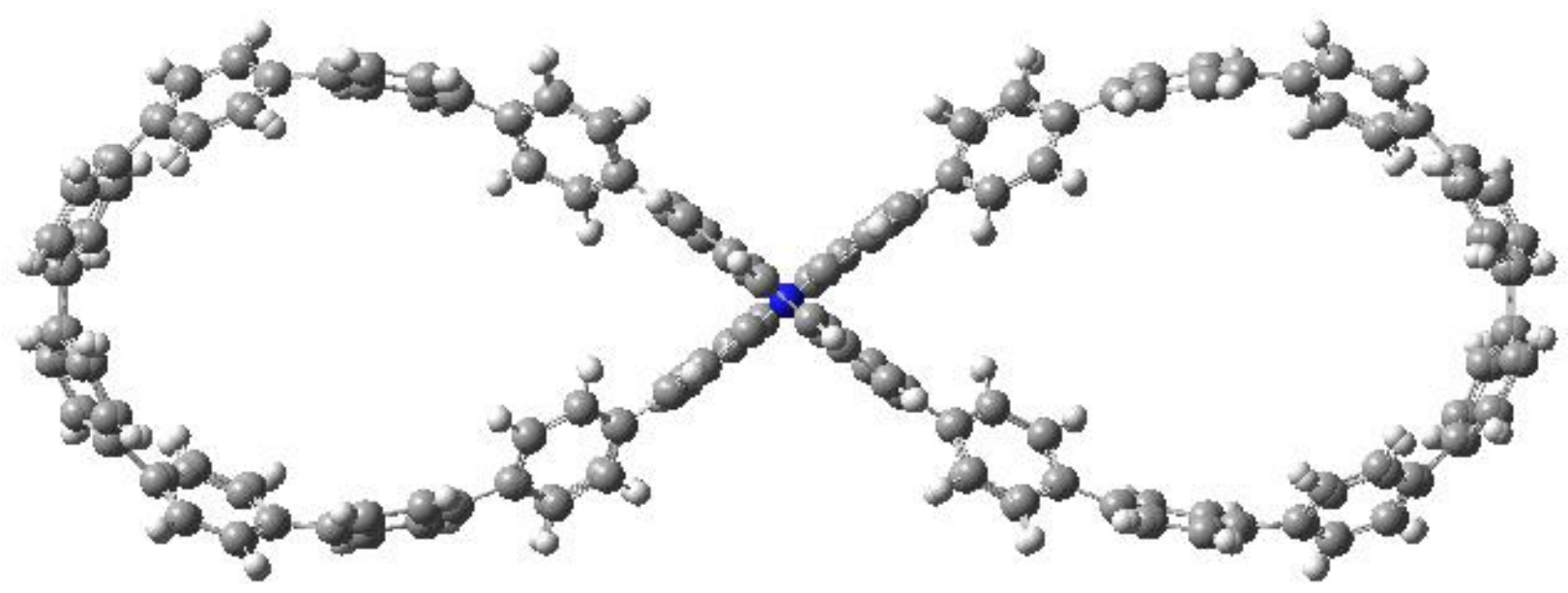

Figure S1. Overlay of the geometry of 1-8 computed at BP86-D3/6-311G(d) and $\omega B 97 X-D / 6-311 G(d)$.

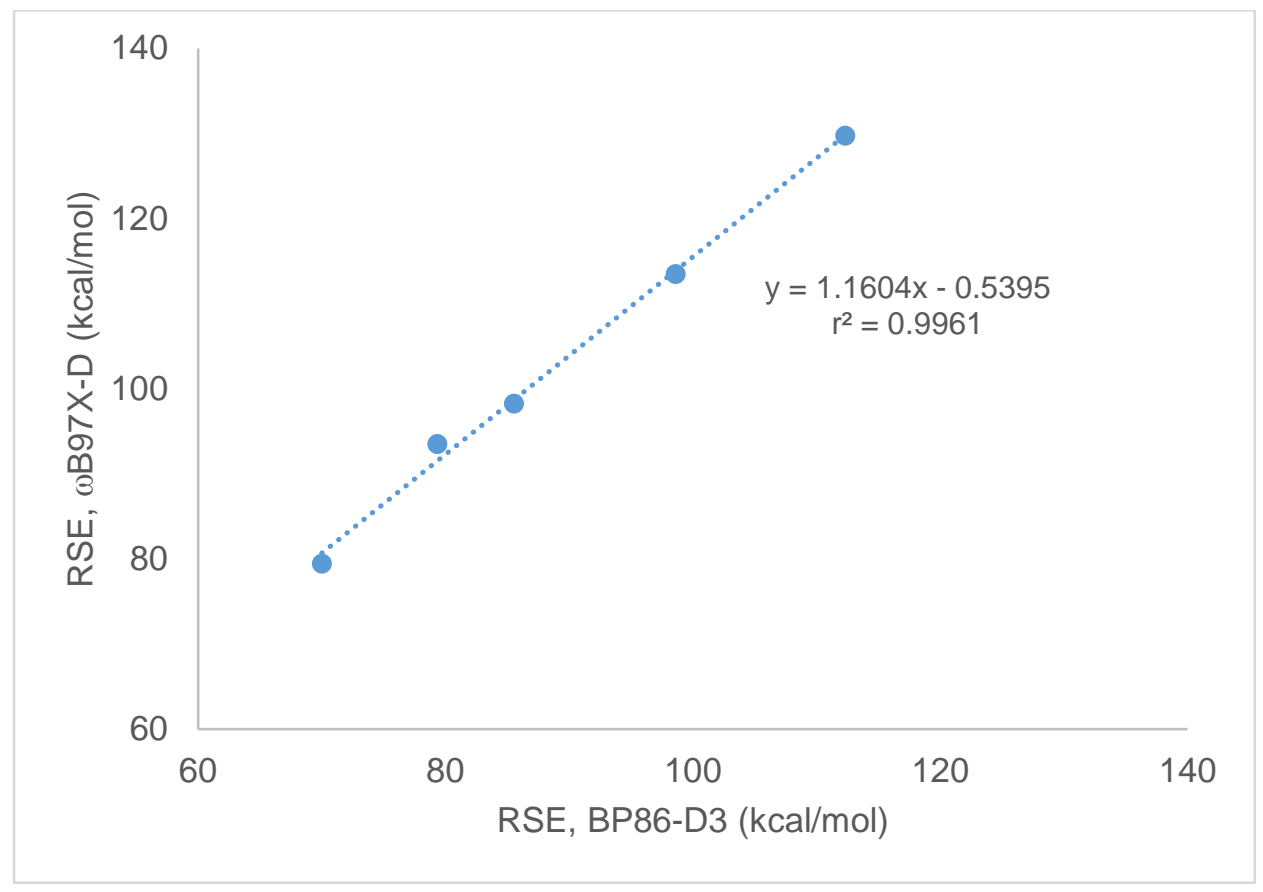

Figure S2. Comparison of the RSE of 1-4 - 1-8 computed at BP86-D3/6-311G(d) and $\omega B$ 97X-D?6-311G(d). 


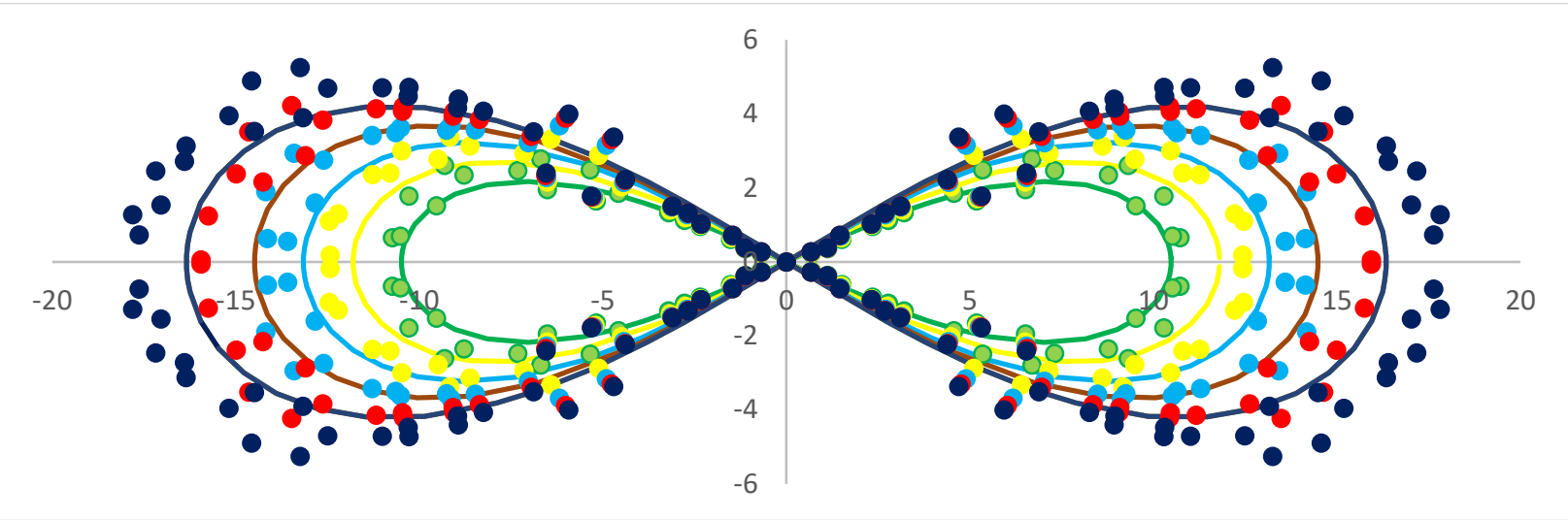

Figure S3. Projection of the heavy atom coordinates of 2-4 (green), 2-5 (yellow), 2-6 (light blue), 2-7

(red) and 2-8 (dark blue) along with best fit Booth lemniscates curves.

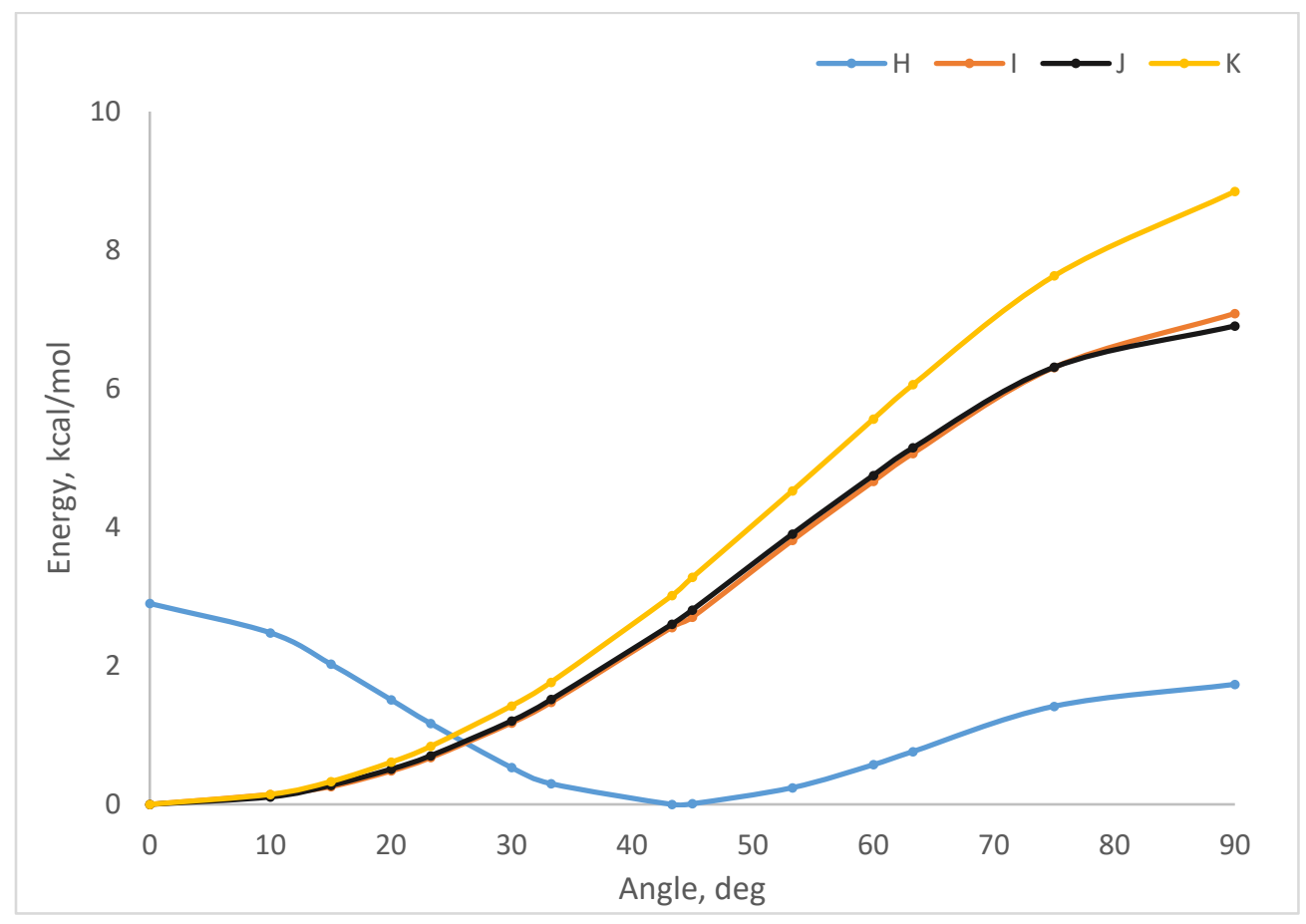

Figure S4. Dihedral rotational energy curves for H-K. 


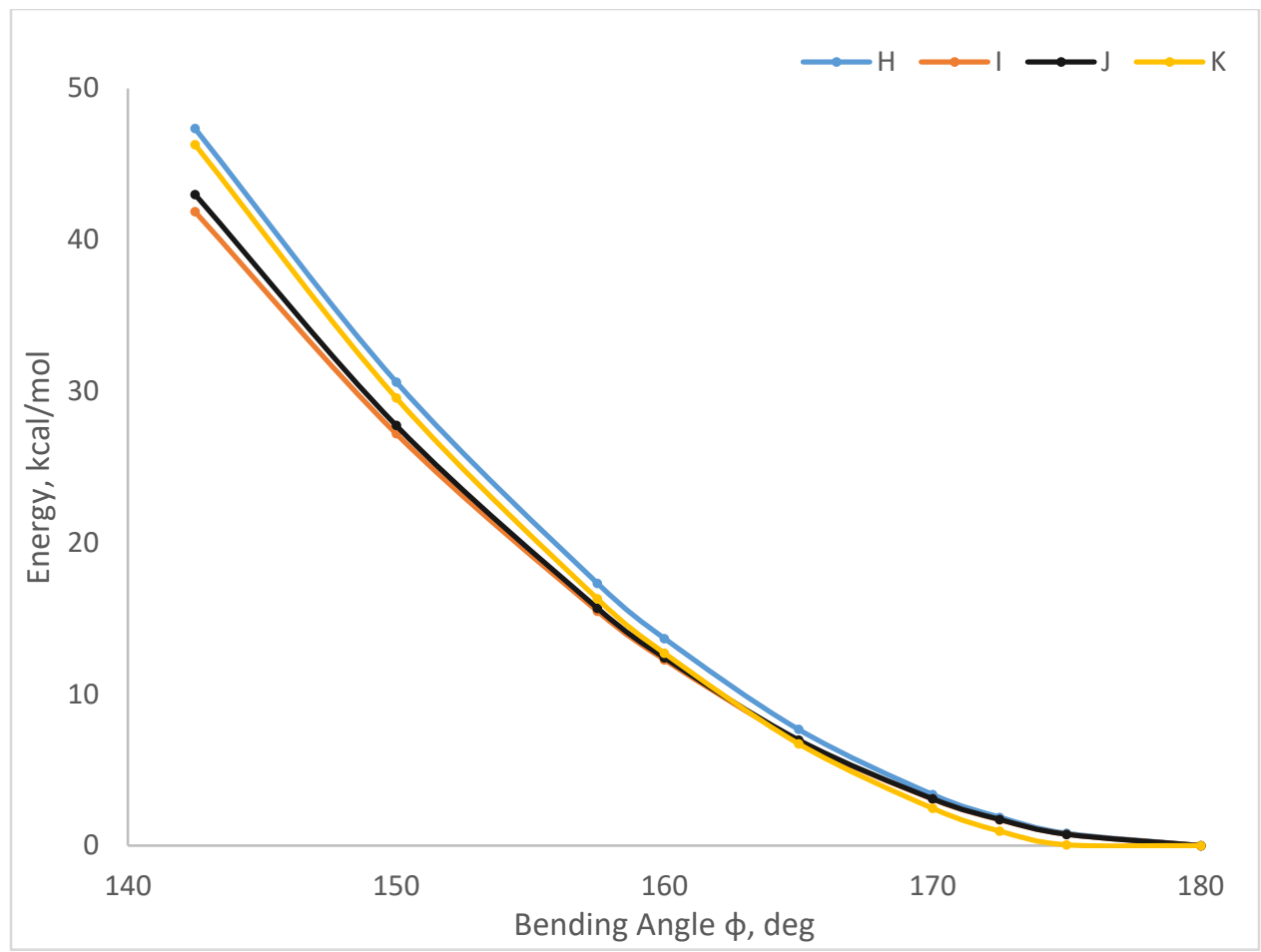

Figure S5. Bending ( $\phi)$ energy curves for H-K. 
Table S1. Ring Strain Energy ( $\mathrm{ccal} \mathrm{mol}^{-1}$ ) for 5-10.

\begin{tabular}{|c|c|c|c|}
\hline & RSE & & RSE \\
\hline $5 N N-4$ & 103.4 & $5 C C-4$ & 113.9 \\
\hline $5 N N-5$ & 89.6 & $5 C C-5$ & 101.2 \\
\hline $5 N N-6$ & 78.4 & $5 C C-6$ & 90.1 \\
\hline 5NN-7 & 69.3 & $5 C C-7$ & 80.5 \\
\hline $5 N N-8$ & 61.3 & $5 C C-8$ & 71.8 \\
\hline $6 N N-4$ & 117.7 & $6 C C-4$ & 129.4 \\
\hline $6 N N-6$ & 92.1 & $6 C C-6$ & 105.0 \\
\hline $6 N N-8$ & 74.3 & $6 C C-8$ & 86.7 \\
\hline $7 N N-4$ & 96.5 & 7CC-4 & 99.1 \\
\hline 7NN-5 & 84.5 & $7 C C-5$ & 88.5 \\
\hline $7 N N-6$ & 74.6 & $7 C C-6$ & 78.9 \\
\hline 7NN-7 & 66.1 & $7 \mathrm{CC}-7$ & 70.8 \\
\hline $7 N N-8$ & 59.2 & $7 C C-8$ & 63.4 \\
\hline $8 N N-4$ & 112.5 & $8 C C-4$ & 114.4 \\
\hline $8 N N-6$ & 90.3 & $8 C C-6$ & 94.5 \\
\hline $8 N N-8$ & 74.3 & $8 C C-8$ & 78.8 \\
\hline $9 N N-5$ & 101.2 & $9 C C-5$ & 112.5 \\
\hline 9NN-7 & 81.3 & $9 \mathrm{CC}-7$ & 92.3 \\
\hline $10 \mathrm{NN}-5$ & 101.1 & $10 C C-5$ & 112.4 \\
\hline $10 N N-7$ & 81.3 & $10 C C-7$ & 92.3 \\
\hline
\end{tabular}




\section{Optimized geometries and corrected enthalpies (hartrees) at $\omega B$ 97X-D/6-311g(d). All compounds have no imaginary frequencies.}

\begin{tabular}{|c|c|c|c|}
\hline \multicolumn{4}{|c|}{$1-4$} \\
\hline $\mathrm{C}$ & -8.514045 & 2.599197 & 1.075665 \\
\hline $\mathrm{C}$ & -9.073129 & 2.665346 & -0.203695 \\
\hline $\mathrm{C}$ & -10.015323 & 1.731793 & $3-0.601327$ \\
\hline $\mathrm{C}$ & -10.412147 & 0.702059 & 0.265589 \\
\hline $\mathrm{C}$ & -10.174021 & 0.932247 & 1.628424 \\
\hline $\mathrm{C}$ & -9.218794 & 1.856853 & 2.027741 \\
\hline $\mathrm{C}$ & -7.053386 & 2.802003 & 1.268296 \\
\hline $\mathrm{C}$ & -6.428451 & 3.291761 & 2.415542 \\
\hline $\mathrm{C}$ & -5.078771 & 3.047938 & 2.648663 \\
\hline $\mathrm{C}$ & -4.307523 & 2.284717 & 1.762869 \\
\hline $\mathrm{C}$ & -4.913935 & 1.893004 & 0.567099 \\
\hline $\mathrm{C}$ & -6.248315 & 2.153375 & 0.323013 \\
\hline $\mathrm{H}$ & -8.635499 & 3.321524 & -0.949675 \\
\hline $\mathrm{H}$ & -10.266603 & 1.691396 & $6-1.654628$ \\
\hline $\mathrm{H}$ & -10.559238 & 0.249486 & $6 \quad 2.377431$ \\
\hline $\mathrm{H}$ & -8.879783 & 1.842819 & 3.059238 \\
\hline $\mathrm{H}$ & -7.007956 & 3.833676 & 3.156844 \\
\hline $\mathrm{H}$ & -4.632062 & 3.433276 & 3.559303 \\
\hline $\mathrm{H}$ & -4.373011 & 1.288610 & -0.152644 \\
\hline $\mathrm{H}$ & -6.705513 & 1.735140 & -0.566811 \\
\hline C & 8.514045 & -2.599197 & 1.075665 \\
\hline $\mathrm{C}$ & 9.073129 & -2.665346 & -0.203695 \\
\hline $\mathrm{C}$ & 10.015323 & -1.731793 & -0.601327 \\
\hline C & 10.412147 & -0.702059 & 0.265589 \\
\hline C & 10.174021 & -0.932247 & 1.628424 \\
\hline C & 9.218794 & -1.856853 & 2.027741 \\
\hline $\mathrm{C}$ & 7.053386 & -2.802003 & 1.268296 \\
\hline C & 6.428451 & -3.291761 & 2.415542 \\
\hline C & 5.078771 & -3.047938 & 2.648663 \\
\hline C & 4.307523 & -2.284717 & 1.762869 \\
\hline C & 4.913935 & -1.893004 & 0.567099 \\
\hline C & 6.248315 & -2.153375 & 0.323013 \\
\hline $\mathrm{H}$ & 8.635499 & -3.321524 & -0.949675 \\
\hline $\mathrm{H}$ & 10.266603 & -1.691396 & -1.654628 \\
\hline $\mathrm{H}$ & 10.559238 & -0.249486 & 2.377431 \\
\hline $\mathrm{H}$ & 8.879783 & -1.842819 & 3.059238 \\
\hline $\mathrm{H}$ & 7.007956 & -3.833676 & 3.156844 \\
\hline $\mathrm{H}$ & 4.632062 & -3.433276 & 3.559303 \\
\hline $\mathrm{H}$ & 4.373011 & -1.288610 & -0.152644 \\
\hline $\mathrm{H}$ & 6.705513 & -1.735140 & -0.566811 \\
\hline C & 8.514045 & 2.599197 & -1.075665 \\
\hline C & 9.073129 & 2.665346 & 0.203695 \\
\hline C & 10.015323 & 1.731793 & 0.601327 \\
\hline C & 10.412147 & 0.702059 & -0.265589 \\
\hline C & 10.174021 & 0.932247 & -1.628424 \\
\hline $\mathrm{C}$ & 9.218794 & 1.856853 & -2.027741 \\
\hline C & 7.053386 & 2.802003 & -1.268296 \\
\hline C & 6.428451 & 3.291761 & -2.415542 \\
\hline $\mathrm{C}$ & 5.078771 & 3.047938 & -2.648663 \\
\hline
\end{tabular}

\begin{tabular}{|c|c|c|c|}
\hline $\mathrm{C}$ & 4.307523 & 2.284717 & -1.762869 \\
\hline $\mathrm{C}$ & 4.913935 & 1.893004 & -0.567099 \\
\hline C & 6.248315 & 2.153375 & -0.323013 \\
\hline $\mathrm{H}$ & 8.635499 & 3.321524 & 0.949675 \\
\hline $\mathrm{H}$ & 10.266603 & 1.691396 & 1.654628 \\
\hline $\mathrm{H}$ & 10.559238 & 0.249486 & -2.377431 \\
\hline $\mathrm{H}$ & 8.879783 & 1.842819 & -3.059238 \\
\hline $\mathrm{H}$ & 7.007956 & 3.833676 & -3.156844 \\
\hline $\mathrm{H}$ & 4.632062 & 3.433276 & -3.559303 \\
\hline $\mathrm{H}$ & 4.373011 & 1.288610 & 0.152644 \\
\hline $\mathrm{H}$ & 6.705513 & 1.735140 & 0.566811 \\
\hline C & -8.514045 & -2.599197 & -1.075665 \\
\hline 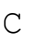 & -9.073129 & -2.665346 & 0.203695 \\
\hline$C$ & -10.015323 & -1.731793 & 0.60 \\
\hline C & -10.412147 & -0.702059 & -0.265589 \\
\hline C & -10.1 & -0.932247 & -1.62 \\
\hline$C$ & -9.218794 & -1.856853 & -2.027 \\
\hline C & -7.053386 & -2.802003 & -1.268 \\
\hline $\mathrm{C}$ & -6.42 & -3.291761 & $-2 \cdot 4$ \\
\hline $\mathrm{C}$ & -5.078771 & -3.047938 & -2.648663 \\
\hline $\mathrm{C}$ & -4.30 & -2.284 & $-1 \cdot 7$ \\
\hline $\mathrm{C}$ & -4.913935 & -1.893004 & -0.56 \\
\hline $\mathrm{C}$ & -6.24 & -2.15 & -0.3 \\
\hline $\mathrm{H}$ & -8.63 & -3.321524 & 0.9 \\
\hline $\mathrm{H}$ & -10.2 & -1.691396 & 1 . \\
\hline $\mathrm{H}$ & -10.5 & -0.24 & \\
\hline $\mathrm{H}$ & -8.87 & -1.84 & -3.0 \\
\hline $\mathrm{H}$ & -7.00 & -3.83 & -3.1 \\
\hline $\mathrm{H}$ & -4.63 & -3.43 & -3.5 \\
\hline $\mathrm{H}$ & -4.37 & -1.288610 & 0 . \\
\hline $\mathrm{H}$ & -6.705 & -1.73 & 0.5 \\
\hline $\mathrm{C}$ & 2.9 & -1.75 & 2.1 \\
\hline $\mathrm{C}$ & 2.08 & $-1 \cdot 32$ & 1.1 \\
\hline $\mathrm{C}$ & 0.94 & -0.6 & 1.4 \\
\hline $\mathrm{C}$ & 0.611 & -0.388139 & 2.826404 \\
\hline $\mathrm{C}$ & 1.45 & -0.86 & 3.82 \\
\hline $\mathrm{C}$ & 2.622129 & -1.525255 & 3.458945 \\
\hline $\mathrm{C}$ & -0.611134 & 0.388139 & 2.82 \\
\hline $\mathrm{C}$ & -0.947 & 0.630499 & 1.483637 \\
\hline $\mathrm{C}$ & -2.08 & 1.326079 & 1.1 \\
\hline $\mathrm{C}$ & -2.962588 & 1.752648 & 2.11 \\
\hline $\mathrm{C}$ & -2.622129 & 1.525255 & 3.4 \\
\hline $\mathrm{C}$ & -1.459494 & 0.867662 & 3.82 \\
\hline $\mathrm{N}$ & 0.000 & 0.000000 & 0.67 \\
\hline $\mathrm{H}$ & 2.292558 & -1.503458 & 0.06 \\
\hline $\mathrm{H}$ & 1.235719 & -0.694127 & 4.8 \\
\hline $\mathrm{H}$ & 3.313086 & -1.823880 & 4.23 \\
\hline $\mathrm{H}$ & -2.292558 & 1.50 & 0.0 \\
\hline $\mathrm{H}$ & -3.313 & 1.823880 & 4.23 \\
\hline $\mathrm{H}$ & -1.23 & 4127 & 4.8 \\
\hline $\mathrm{C}$ & 2.962588 & 1.752648 & -2.111198 \\
\hline $\mathrm{C}$ & 2.08 & 1.326079 & $-1 \cdot 1$ \\
\hline $\mathrm{C}$ & 0.947 & 0.630499 & $-1 \cdot 48$ \\
\hline $\mathrm{C}$ & 0.61 & 0.388139 & -2.826404 \\
\hline $\mathrm{C}$ & 1.45 & 0.867662 & -3.82 \\
\hline $\mathrm{C}$ & 2.622 & 1.525255 & $-3 \cdot 4$ \\
\hline , & -0.611134 & -0.388139 & -2.82 \\
\hline
\end{tabular}


$\begin{array}{lrrr}\mathrm{C} & -0.947391 & -0.630499 & -1.483637 \\ \mathrm{C} & -2.086659 & -1.326079 & -1.109815 \\ \mathrm{C} & -2.962588 & -1.752648 & -2.111198 \\ \mathrm{C} & -2.622129 & -1.525255 & -3.458945 \\ \mathrm{C} & -1.459494 & -0.867662 & -3.820512 \\ \mathrm{~N} & 0.000000 & 0.000000 & -0.679910 \\ \mathrm{H} & 2.292558 & 1.503458 & -0.061036 \\ \mathrm{H} & 1.235719 & 0.694127 & -4.867959 \\ \mathrm{H} & 3.313086 & 1.823880 & -4.238539 \\ \mathrm{H} & -2.292558 & -1.503458 & -0.061036 \\ \mathrm{H} & -3.313086 & -1.823880 & -4.238539 \\ \mathrm{H} & -1.235719 & -0.694127 & -4.867959 \\ \Delta \mathrm{H}=-2878.150399 & \end{array}$

\section{1-5}

\begin{tabular}{|c|c|c|c|}
\hline $\mathrm{C}$ & -8.333793 & 3.458554 & 1.162655 \\
\hline C & -8.908579 & 3.539581 & -0.107180 \\
\hline $\mathrm{C}$ & -10.191092 & 3.072364 & -0.333354 \\
\hline C & -10.950984 & 2.503112 & 0.698345 \\
\hline $\mathrm{C}$ & -10.479532 & 2.712732 & 2.000851 \\
\hline C & -9.188486 & 3.169197 & 2.228791 \\
\hline C & -6.864033 & 3.324607 & 1.318973 \\
\hline $\mathrm{C}$ & -6.123823 & 3.837307 & 2.385082 \\
\hline C & -4.814390 & 3.426832 & 2.604121 \\
\hline C & -4.200861 & 2.477864 & 1.778767 \\
\hline $\mathrm{C}$ & -4.918787 & 2.028523 & 0.668504 \\
\hline $\mathrm{C}$ & -6.217789 & 2.446014 & 0.442270 \\
\hline $\mathrm{H}$ & -8.302812 & 3.851941 & -0.952053 \\
\hline $\mathrm{H}$ & -10.529609 & 3.013917 & $-1 \cdot 3$ \\
\hline $\mathrm{H}$ & -11.058545 & 2.377659 & 2.85 \\
\hline $\mathrm{H}$ & -8.797295 & 3.152648 & 3.24 \\
\hline $\mathrm{H}$ & -6.581333 & 4.549872 & 3.064 \\
\hline $\mathrm{H}$ & -4.267069 & 3.841975 & 3.44 \\
\hline $\mathrm{H}$ & -4.489778 & 1.28 & \\
\hline $\mathrm{H}$ & -6.774669 & 2.007870 & -0.37902 \\
\hline $\mathrm{C}$ & 8.333793 & -3.458554 & \\
\hline $\mathrm{C}$ & 8.908579 & -3.539581 & -0.10718 \\
\hline $\mathrm{C}$ & 10.191092 & -3.072364 & -0.3 \\
\hline C & 10.950984 & -2.503112 & 0.69834 \\
\hline $\mathrm{C}$ & 10.479532 & -2.712732 & \\
\hline $\mathrm{C}$ & 9.188486 & -3.169197 & 2.22879 \\
\hline $\mathrm{C}$ & 6.864033 & -3.324607 & 1.3 \\
\hline $\mathrm{C}$ & 6.123823 & -3.837307 & 2.38 \\
\hline $\mathrm{C}$ & 4.814390 & -3.426832 & 2.6 \\
\hline $\mathrm{C}$ & 4.200861 & -2.477864 & 1.778767 \\
\hline $\mathrm{C}$ & 4.918787 & -2.028523 & 0.668504 \\
\hline $\mathrm{C}$ & 6.217789 & -2.446014 & 0.442270 \\
\hline $\mathrm{H}$ & 8.302812 & -3.851941 & -0.952053 \\
\hline $\mathrm{H}$ & 10.529609 & -3.013917 & -1.36059 \\
\hline $\mathrm{H}$ & 11.058545 & -2.377659 & 2.85424 \\
\hline $\mathrm{H}$ & 8.797295 & -3.152648 & 3.24154 \\
\hline $\mathrm{H}$ & 6.581333 & -4.549872 & 3.064792 \\
\hline $\mathrm{H}$ & 4.267069 & -3.841975 & 3.44433 \\
\hline $\mathrm{H}$ & 9778 & -1.281150 & 0.008986 \\
\hline 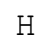 & 6.774669 & -2.007870 & -0.379026 \\
\hline
\end{tabular}

\begin{tabular}{|c|c|c|c|}
\hline C & 8.333793 & 3.458554 & -1.162655 \\
\hline 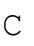 & 8.908579 & 3.539581 & 0.107180 \\
\hline C & 10.191092 & 3.072364 & 0.333354 \\
\hline C & 10.950984 & 2.503112 & -0.6 \\
\hline C & 10.479532 & 2.712732 & -2.000851 \\
\hline $\mathrm{C}$ & 9.188486 & 3.169197 & -2.228791 \\
\hline C & 11.855211 & 1.358978 & -0.374433 \\
\hline C & 6.864033 & 3.324607 & -1.318973 \\
\hline C & 6.123823 & 3.837307 & $-2 \cdot 3$ \\
\hline 0 & 4.814390 & 3.426832 & -2.60 \\
\hline C & 4.200861 & 2.477864 & -1.778767 \\
\hline 0 & 4.918787 & 2.028523 & -0.668504 \\
\hline C & 6.217789 & 2.44 & -0.4 \\
\hline C & 12.065431 & 0.350740 & -1.32 \\
\hline C & 12.08 & -0.98 & -0 \\
\hline 0 & 11.85 & -1.358 & $0.3^{\circ}$ \\
\hline 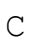 & 12.06 & -0.35 & 1. \\
\hline 0 & 12.08 & 0.983967 & 0.9 \\
\hline $\mathrm{H}$ & 8.30 & 3. & 0 \\
\hline H & 10.52 & 3.01 & 1.3 \\
\hline $\mathrm{H}$ & 11.0 & 2 & -2. \\
\hline H & 8.797295 & 3.15 & -3.2 \\
\hline 1 & 6.58 & 4.5 & -3 \\
\hline P & 4.26 & 75 & -3 . \\
\hline 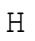 & 4.48 & $1.2 \varepsilon$ & -0.0 \\
\hline 有 & 6.77 & & \\
\hline H & 12.03 & $0.5^{\circ}$ & $-2 \cdot 3 \xi$ \\
\hline 形 & 12.0 & $-1 \cdot 7$ & -1.7 \\
\hline H & 12.032217 & -0.57 & 2.3 \\
\hline & 12.0 & 1.72 & \\
\hline 0 & -8.333793 & -3.458554 & -1.16 \\
\hline C & -8.9 & -3.5 & 0.1 \\
\hline 0 & -10.191092 & -3.072364 & 354 \\
\hline C & -10 & -2.503112 & -0 . \\
\hline C & -10.47 & -2.712732 & -2.0 \\
\hline 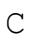 & -9.1 & -3.16 & $-2 \cdot 2$ \\
\hline & -11.8 & $-1 \cdot 3$ & -0 \\
\hline C & -6.8 & -3.32 & $-1 \cdot 3$ \\
\hline 0 & -6.123 & -3.837307 & -2.38 \\
\hline C & -4.8 & -3.426 & $-2 \cdot 6$ \\
\hline $\mathrm{C}$ & -4.200 & -2.477864 & -1.77 \\
\hline C & -4.918 & -2.028523 & -0.66 \\
\hline C & -6.217 & -2.446014 & -0.442 \\
\hline C & -12.06 & -0.350740 & -1.3 \\
\hline C & -12.083763 & 0.983967 & -0. \\
\hline C & -11.85 & 1.358978 & 0. \\
\hline C & -12.06 & 0.350740 & 1.3 \\
\hline C & -12.0 & -0.983967 & 0 . \\
\hline $\mathrm{H}$ & -8.302 & -3.851941 & 0.95 \\
\hline $\mathrm{H}$ & -10.5 & -3.013917 & 1. \\
\hline & -11.0 & -2.377659 & -2.8 \\
\hline F & -8.79 & -3.152648 & -3.24 \\
\hline & -6.581 & -4.549872 & -3.06 \\
\hline $\mathrm{F}$ & -4.26 & -3.841975 & -3.44 \\
\hline $\mathrm{F}$ & -4.489778 & -1.281150 & -0.0 \\
\hline & -6.7 & -2.007870 & 0.3 \\
\hline & -12 & -0.5 & $3-2$. \\
\hline
\end{tabular}




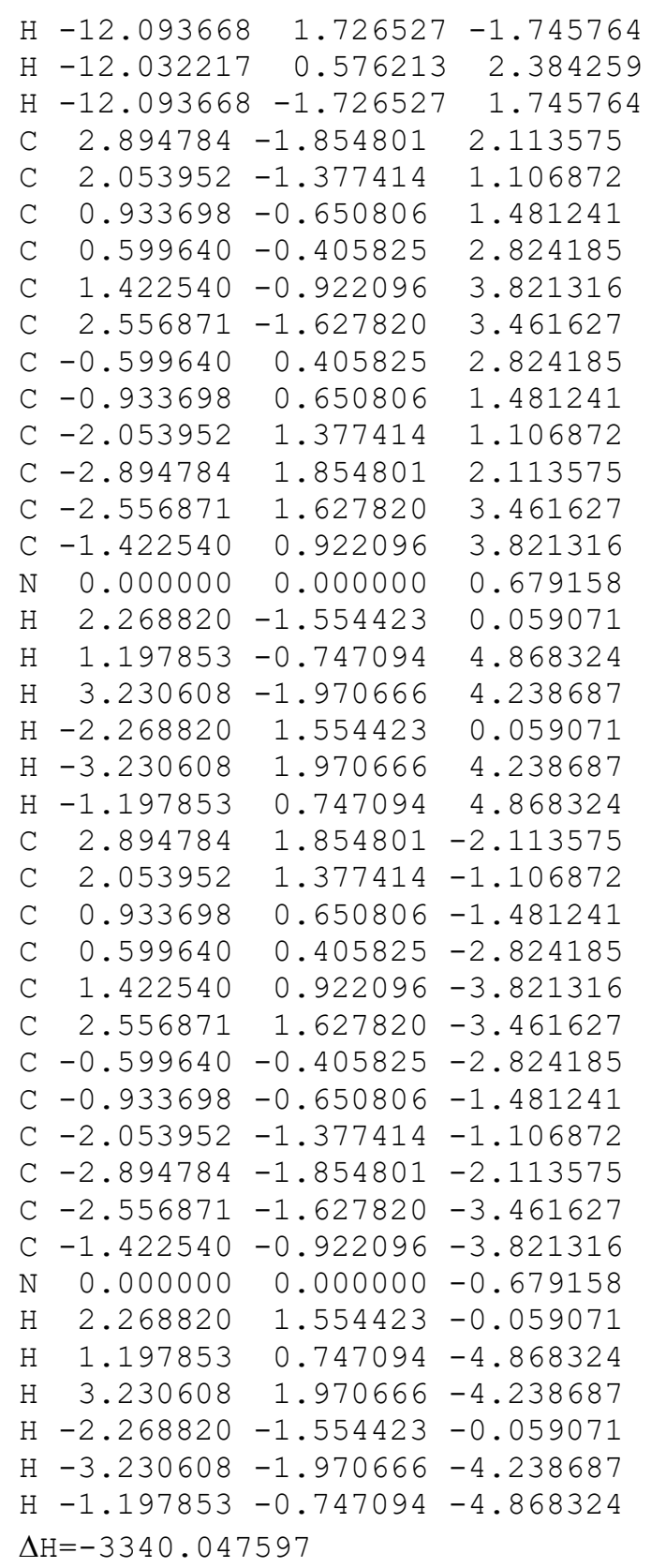

\section{1-6}

$\begin{array}{lrll}\text { C } & 8.120410 & 1.332333 & 4.045642 \\ \text { C } & 8.785911 & 0.104988 & 4.043357 \\ \text { C } & 10.159638 & 0.043744 & 3.877879 \\ \text { C } & 10.921911 & 1.206565 & 3.722007 \\ \text { C } & 10.280661 & 2.428324 & 3.947622 \\ \text { C } & 8.902290 & 2.490032 & 4.097009 \\ \text { C } & 12.192949 & 1.100044 & 2.958019 \\ \text { C } & 6.671572 & 1.413887 & 3.735206 \\ \text { C } & 5.818758 & 2.381736 & 4.269759 \\ \text { C } & 4.542582 & 2.568812 & 3.755995 \\ \text { C } & 4.071967 & 1.799878 & 2.686906\end{array}$

\begin{tabular}{|c|c|c|c|}
\hline C & 4.896103 & 0.779882 & 2.208708 \\
\hline C & 6.168180 & 0.592074 & 2.721744 \\
\hline $\mathrm{C}$ & 12.368122 & 1.978540 & 1.886437 \\
\hline & 13.081484 & 1.595189 & 0.763633 \\
\hline $\mathrm{C}$ & 13.646426 & 0.320296 & 0.672782 \\
\hline & 13.725329 & -0.421744 & 96 \\
\hline C & 13.005084 & -0.040746 & 2.981987 \\
\hline & 8.213603 & -0.816810 & 4.072775 \\
\hline & 10.624673 & -0.92 & 3. \\
\hline & 10.8479 & 3.352650 & 3 \\
\hline & 8.41 & 3.46 & \\
\hline & 6.162244 & 3.00 & 5 . \\
\hline & 3.9 & 3.31 & \\
\hline & 4.56 & 0.17 & 1. \\
\hline & 6.81 & -0.15 & \\
\hline & 11.7 & 2.89 & 1. \\
\hline & 13. & 2.22 & -0 . \\
\hline & 14.2 & -1.37 & 1. \\
\hline & 12.9 & -0.6 & \\
\hline C & -8.1 & 1.32 & -4 . \\
\hline & -8.7 & 0.1 & -4 . \\
\hline & -10.1 & 0.040285 & -3.8 \\
\hline C & -10.9 & 1.202867 & -3 \\
\hline C & -10.28 & 2.4 & -3 \\
\hline C & -8.90 & $2.4 \xi$ & -4.09 \\
\hline & -12 & & 99 \\
\hline $\mathrm{C}$ & -6.67 & 1.4. & -3.7 \\
\hline C & -5 & & -4 . \\
\hline $\mathrm{C}$ & -4.542 & 2.5 & -3.7 \\
\hline C & -4 & & -2 . \\
\hline C & -4.896 & $0.7^{7}$ & -2.20 \\
\hline C & -6 & 0.5 & $-2 \cdot 7$ \\
\hline C & -12.36 & 4403 & $-1 . \varepsilon$ \\
\hline C & -13 & 351 & -0 \\
\hline C & -13.64 & 0.315796 & -0.6 \\
\hline C & -13 & -0.426267 & $-1 . \varepsilon$ \\
\hline & -13.00 & -0.045069 & -2.9 \\
\hline & -8.212 & -0.81 & -4.0 \\
\hline & -10.6 & -0.9 & -3 \\
\hline$F$ & -10.8 & 3.3 & -3 \\
\hline & -8.41 & 3.45 & -4.13 \\
\hline $\mathrm{F}$ & -6.16 & 3.00 & -5.0 \\
\hline & -3.90 & 3.33 & -4.18 \\
\hline $\mathrm{H}$ & -4.5 & 0.16 & $-1 \cdot 37$ \\
\hline & -6.8 & -0.15 & -2.26 \\
\hline & -11 & 2.8 & -1 \\
\hline $\mathrm{H}$ & -13.02 & 2.217783 & 0.1 \\
\hline $\mathrm{H}$ & -14.2 & $-1 \cdot 3$ & -1. \\
\hline $\mathrm{H}$ & -12.98 & -0.704181 & -3.84 \\
\hline $\mathrm{C}$ & -8.12 & -1.332 & 4.0 \\
\hline$\Omega$ & -8.78 & -0.104 & 4.04 \\
\hline $\mathrm{C}$ & -10.15 & -0.043744 & 3.877879 \\
\hline 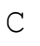 & -10.921911 & -1.206565 & 3.722007 \\
\hline $\mathrm{C}$ & $-10.2 \varepsilon$ & -2.428324 & 3.947622 \\
\hline $\mathrm{C}$ & -8.9 & -2.490032 & 4.097009 \\
\hline 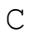 & -12 & -1 . & 58019 \\
\hline & -6 & -1.4 & \\
\hline
\end{tabular}




\begin{tabular}{|c|c|c|c|}
\hline C & -5.818758 & -2.381736 & 4.269759 \\
\hline C & -4.542582 & -2.568812 & 3.755995 \\
\hline C & -4.071967 & -1.799878 & 2.686906 \\
\hline $\mathrm{C}$ & -4.896103 & -0.779882 & 2.208708 \\
\hline $\mathrm{C}$ & -6.168180 & -0.592074 & 2.721744 \\
\hline $\mathrm{C}$ & -12.368122 & -1.978540 & 1.886437 \\
\hline $\mathrm{C}$ & -13.081484 & -1.595189 & 0.7 \\
\hline $\mathrm{C}$ & -13.646426 & -0.320296 & 0.672782 \\
\hline $\mathrm{C}$ & -13.725329 & 0.421744 & \\
\hline $\mathrm{C}$ & -13.005084 & 0.040746 & 2.9 \\
\hline 1 & -8.213603 & 0.816810 & 4.072775 \\
\hline $\mathrm{H}$ & -10.624673 & 0.925637 & 3 . \\
\hline $\mathrm{H}$ & -10.847940 & -3.352650 & \\
\hline $\mathrm{H}$ & -8.419303 & -3.461515 & 4.137581 \\
\hline $\mathrm{H}$ & -6.162244 & -3.009 & 5.08 \\
\hline $\mathrm{H}$ & -3.905326 & -3.335401 & 4.185446 \\
\hline $\mathrm{H}$ & -4.569819 & -0.170 & 1.37 \\
\hline $\mathrm{H}$ & -6.813710 & 0.151494 & 2.26653 \\
\hline $\mathrm{H}$ & $-11 \cdot 78$ & -2.89 & $1 . \varepsilon$ \\
\hline $\mathrm{H}$ & -13.020039 & -2.22 & -0 . \\
\hline $\mathrm{H}$ & -14.24 & 1.37 & \\
\hline $\mathrm{H}$ & -12.985284 & 0.699850 & 3.8 \\
\hline C & 8.120 & $-1 \cdot 32$ & -4.04 \\
\hline C & 8.785469 & -0.101972 & -4.04 \\
\hline C & 10.15 & -0.04 & -3.87 \\
\hline C & 10.92 & -1.202 & -3.72 \\
\hline C & 10.28 & -2.42 & -3.94 \\
\hline C & 8.90 & -2.48 & -4.09 \\
\hline $\mathrm{C}$ & 12.192 & -1.095 & -2.95 \\
\hline C & 6.67 & -1.41 & -3.7 \\
\hline $\mathrm{C}$ & 5.81 & -2.37 & -4.2697 \\
\hline $\mathrm{C}$ & 4.54 & -2.56 & -3.7 \\
\hline $\mathrm{C}$ & 4.07 & -1.79 & -2.686 \\
\hline $\mathrm{C}$ & 4.89 & -0.778 & $-2 \cdot 2$ \\
\hline $\mathrm{C}$ & 6.168084 & -0.590092 & -2.7213 \\
\hline $\mathrm{C}$ & 12.368478 & -1.97 & -1.8 \\
\hline $\mathrm{C}$ & 13.081 & -1.59 & -0.764 \\
\hline $\mathrm{C}$ & 13.64 & -0.31 & -0.67 \\
\hline $\mathrm{C}$ & 13.725000 & 0.426 & -1.85 \\
\hline $\mathrm{C}$ & 13.004745 & 0 . & $-2 \cdot 9$ \\
\hline $\mathrm{H}$ & 8.212846 & 634 & -4.07260 \\
\hline $\mathrm{H}$ & 10.623929 & 0 . & -3.7 \\
\hline $\mathrm{H}$ & 10.848595 & -3.348962 & -3.899 \\
\hline $\mathrm{H}$ & 8.419953 & -3.458 & -4.13 \\
\hline $\mathrm{H}$ & 6.162557 & -3.006757 & -5.08672 \\
\hline $\mathrm{H}$ & 3.905783 & -3.333681 & -4.18 \\
\hline $\mathrm{H}$ & 4.569754 & -0.169133 & -1.371207 \\
\hline $\mathrm{H}$ & 6.813463 & 0.153562 & -2.26 \\
\hline $\mathrm{H}$ & 11.785008 & -2.887199 & -1.836577 \\
\hline $\mathrm{H}$ & 13.020676 & -2.217783 & 0.118440 \\
\hline $\mathrm{H}$ & 14.249377 & 1.376934 & -1.86944 \\
\hline $\mathrm{H}$ & 12.984652 & 4181 & -3.84509 \\
\hline $\mathrm{C}$ & -2.803337 & 2.116851 & -1.98525 \\
\hline C & -2.003480 & 1.105862 & -1.451960 \\
\hline C & -0.911128 & 1.479670 & -0.68272 \\
\hline C & -0.581585 & 2.822523 & -0.43 \\
\hline $\mathrm{C}$ & -1.368616 & 3.822446 & -0.99556 \\
\hline
\end{tabular}

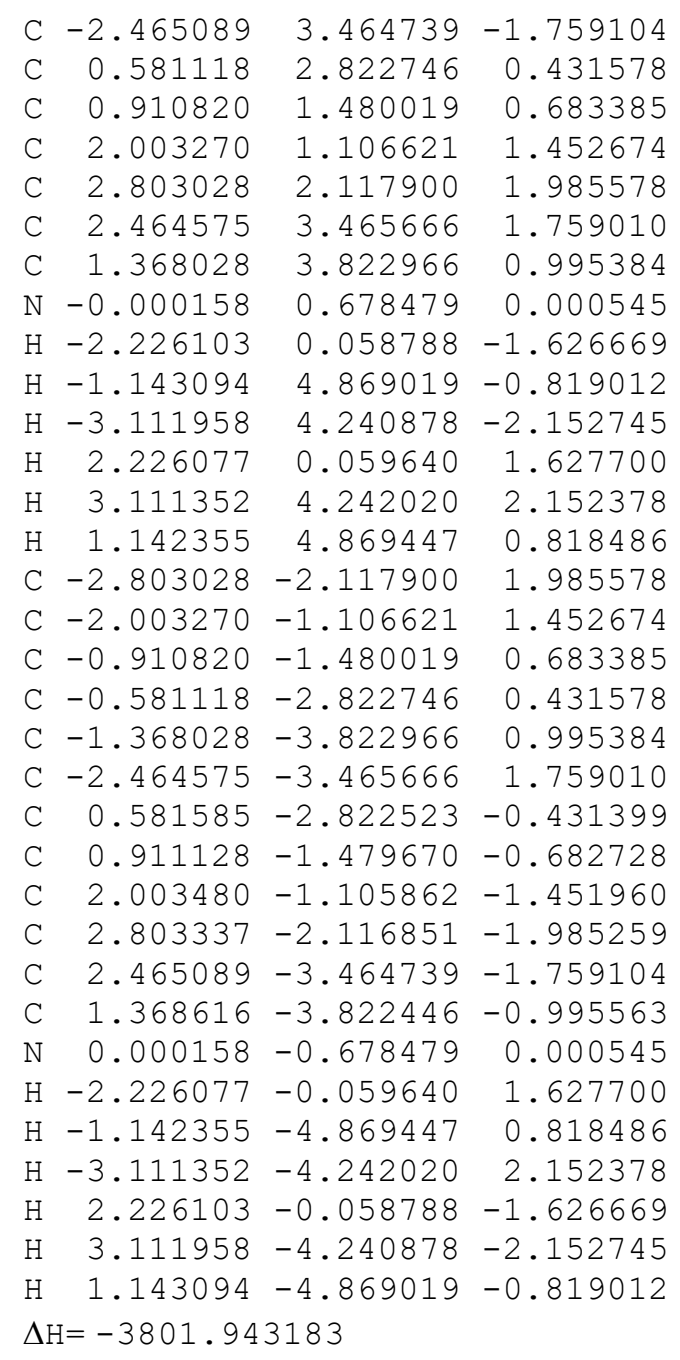

\section{1-7}




\begin{tabular}{|c|c|c|c|}
\hline $\mathrm{H}$ & -10.557270 & 4.463522 & -0.662428 \\
\hline $\mathrm{H}$ & -10.496799 & 4.883366 & 3.601947 \\
\hline & -8.054876 & 4.754865 & 3.575684 \\
\hline & -5.806256 & 5.467704 & 2.980553 \\
\hline 1 & -3.610687 & 4.413257 & 3.268761 \\
\hline & -4.599547 & 1.524668 & 0.262725 \\
\hline 1 & -6.791569 & 2.565788 & -0.015304 \\
\hline & -11.827758 & 2.969812 & 3.239607 \\
\hline & -13.720760 & 1.539935 & 2.866378 \\
\hline & -14.787540 & 3.842847 & $7-0.597237$ \\
\hline & -12.847097 & 5.264158 & $3-0.231076$ \\
\hline & 7.897317 & -4.520356 & 1.442435 \\
\hline & 8.628787 & -4.530154 & 0.253007 \\
\hline & 10.014091 & -4.577704 & 0.270141 \\
\hline & 10.719147 & -4.626528 & 1.476386 \\
\hline & 9.980901 & -4.791328 & 2.651207 \\
\hline & 8.595477 & -4.733197 & 2.634644 \\
\hline C & 12.136180 & -4.184387 & 1.491986 \\
\hline & 6.479230 & -4.084811 & 1.475 \\
\hline C & 5.549829 & -4.598629 & 2.382486 \\
\hline 乙 & 4.308604 & -4.001019 & 2.54 \\
\hline $\mathrm{C}$ & 3.948491 & -2.868651 & 1.810277 \\
\hline$\checkmark$ & 4.844333 & -2.407932 & 0.844524 \\
\hline $\mathrm{C}^{\prime}$ & 6.085151 & -3.002865 & 0.682605 \\
\hline C & 12.495746 & -3.203332 & 2.41 \\
\hline C & 13.587569 & -2.383206 & 2.20 \\
\hline $\mathrm{C}$ & 14.377342 & -2.508541 & 1.05 \\
\hline C & 14.150135 & -3.639676 & 0.25 \\
\hline$C$ & 13.045488 & -4.454561 & 0.46 \\
\hline $\mathrm{H}$ & 8.112744 & -4.424576 & -0.69 \\
\hline $\mathrm{H}$ & 10.557270 & -4.463522 & -0.662428 \\
\hline $\mathrm{H}$ & 10.496799 & -4.883366 & 3.60 \\
\hline $\mathrm{H}$ & 8.054876 & -4.754865 & 3.575684 \\
\hline $\mathrm{H}$ & 5.806256 & -5.467704 & 2.98 \\
\hline $\mathrm{H}$ & 3.610687 & -4.413257 & 3.268761 \\
\hline $\mathrm{H}$ & 4.599547 & -1.524668 & 0.26 \\
\hline $\mathrm{H}$ & 6.791569 & -2.565788 & -0.015304 \\
\hline $\mathrm{H}$ & 11.827758 & -2.969812 & 3.23 \\
\hline $\mathrm{H}$ & 13.720760 & -1.539935 & 2.866378 \\
\hline $\mathrm{H}$ & 14.787540 & -3.842847 & -0.597237 \\
\hline $\mathrm{H}$ & 12.847097 & -5.264158 & -0.231076 \\
\hline $\mathrm{C}$ & 7.897317 & 4.520356 & -1.442435 \\
\hline C & 8.628787 & 4.530154 & -0.253007 \\
\hline $\mathrm{C}$ & 10.014091 & 4.577704 & -0.270141 \\
\hline C & 10.719147 & 4.626528 & -1.476386 \\
\hline $\mathrm{C}$ & 9.980901 & 4.791328 & -2.651207 \\
\hline C & 8.595477 & 4.733197 & -2.634644 \\
\hline $\mathrm{C}$ & 12.136180 & 4.184387 & -1.491986 \\
\hline C & 6.479230 & 4.084811 & -1.475258 \\
\hline $\mathrm{C}$ & 5.549829 & 4.598629 & -2.382486 \\
\hline $\mathrm{C}$ & 4.308604 & 4.001019 & -2.546797 \\
\hline $\mathrm{C}$ & 3.948491 & 2.868651 & -1.810277 \\
\hline $\mathrm{C}$ & 4.844333 & 2.407932 & -0.844524 \\
\hline $\mathrm{C}$ & 6.085151 & 3.002865 & -0.682605 \\
\hline $\mathrm{C}$ & 12.495746 & 3.203332 & -2.417486 \\
\hline $\mathrm{C}$ & 13.587569 & 2.383206 & -2.200175 \\
\hline $\mathrm{C}$ & 14.377342 & 2.508541 & -1.052649 \\
\hline
\end{tabular}




$\begin{array}{lccc}\text { H } & 2.174353 & -1.700809 & 0.058601 \\ \mathrm{H} & 1.099201 & -0.872270 & 4.869073 \\ \mathrm{H} & 3.007586 & -2.295353 & 4.241416 \\ \mathrm{H} & -2.174353 & 1.700809 & 0.058601 \\ \mathrm{H} & -3.007586 & 2.295353 & 4.241416 \\ \mathrm{H} & -1.099201 & 0.872270 & 4.869073 \\ \mathrm{C} & 2.718405 & 2.097584 & -2.118137 \\ \mathrm{C} & 1.951725 & 1.522218 & -1.105207 \\ \mathrm{C} & 0.888480 & 0.712593 & -1.478881 \\ \mathrm{C} & 0.564676 & 0.453042 & -2.821413 \\ \mathrm{C} & 1.322353 & 1.053387 & -3.822777 \\ \mathrm{C} & 2.384053 & 1.864479 & -3.465841 \\ \mathrm{C} & -0.564676 & -0.453042 & -2.821413 \\ \mathrm{C} & -0.888480 & -0.712593 & -1.478881 \\ \mathrm{C} & -1.951725 & -1.522218 & -1.105207 \\ \mathrm{C} & -2.718405 & -2.097584 & -2.118137 \\ \mathrm{C} & -2.384053 & -1.864479 & -3.465841 \\ \mathrm{C} & -1.322353 & -1.053387 & -3.822777 \\ \mathrm{~N} & 0.000000 & 0.000000 & -0.677920 \\ \mathrm{H} & 2.174353 & 1.700809 & -0.058601 \\ \mathrm{H} & 1.099201 & 0.872270 & -4.869073 \\ \mathrm{H} & 3.007586 & 2.295353 & -4.241416 \\ \mathrm{H} & -2.174353 & -1.700809 & -0.058601 \\ \mathrm{H} & -3.007586 & -2.295353 & -4.241416 \\ \mathrm{H} & -1.099201 & -0.872270 & -4.869073 \\ \mathrm{C} & -15.094945 & -1.302918 & -0.551711 \\ \mathrm{C} & -15.094945 & 1.302918 & 0.551711 \\ \mathrm{C} & 15.094945 & -1.302918 & 0.551711 \\ \mathrm{C} & 15.094945 & 1.302918 & -0.551711 \\ \mathrm{C} & -15.275395 & -0.176296 & -1.362729 \\ \mathrm{C} & -15.275395 & 0.176296 & 1.362729 \\ \mathrm{C} & 15.275395 & -0.176296 & 1.362729 \\ \mathrm{C} & 15.275395 & 0.176296 & -1.362729 \\ \mathrm{C} & -15.249784 & -1.099430 & 0.823996 \\ \mathrm{C} & -15.249784 & 1.099430 & -0.823996 \\ \mathrm{C} & 15.249784 & -1.099430 & -0.823996 \\ \mathrm{C} & 15.249784 & 1.099430 & 0.823996 \\ \mathrm{H} & -15.295787 & -0.275018 & -2.442311 \\ \mathrm{H} & -15.204319 & 1.938188 & -1.509623 \\ \mathrm{H} & -15.204319 & -1.938188 & 1.509623 \\ \mathrm{H} & -15.295787 & 0.275018 & 2.442311 \\ \mathrm{H} & 15.295787 & 0.275018 & -2.442311 \\ \mathrm{H} & 15.204319 & -1.938188 & -1.509623 \\ \mathrm{H} & 15.204319 & 1.938188 & 1.509623 \\ \mathrm{H} & 15.295787 & -0.275018 & 2.442311\end{array}$

$\Delta \mathrm{H}=-4263.822102$

\section{$1-8$}

$\begin{array}{llll}\text { C }-7.736419 & 4.820425 & 1.350029 \\ \text { C }-8.442111 & 4.849591 & 0.145434 \\ \text { C }-9.807496 & 5.087378 & 0.124741 \\ \text { C }-10.519031 & 5.315695 & 1.305903 \\ \text { C }-9.788818 & 5.421275 & 2.492149 \\ \text { C }-8.424779 & 5.172675 & 2.514221 \\ \text { C }-11.997315 & 5.183742 & 1.300286\end{array}$

\begin{tabular}{|c|c|c|c|}
\hline $\mathrm{C}$ & -6.359990 & 4.272443 & 1.426558 \\
\hline $\mathrm{C}$ & -5.407696 & 4.760314 & 2.324457 \\
\hline C & -4.200515 & 4.103166 & 2.510959 \\
\hline & -3.897307 & 2.936572 & 1.803772 \\
\hline C & -4.818168 & 2.489954 & 0.855172 \\
\hline & -6.026780 & 3.143178 & 0.673404 \\
\hline C & -12.598551 & 4.396602 & 2.284256 \\
\hline C & -13.882242 & 3.905392 & 2. \\
\hline C & -14.618799 & 4.182453 & 0 . \\
\hline C & -14.0 & 5.113260 & 0. \\
\hline C & -12.7 & 5.602370 & 0.2 \\
\hline & -7.927 & 4.629548 & -0.784407 \\
\hline & $-10 \cdot 34$ & 5.005703 & -0.815577 \\
\hline & $-10 \cdot 30$ & 5.641400 & 3.42 \\
\hline $\mathrm{H}$ & -7.902 & 5.173813 & 3.46 \\
\hline & -5.616374 & 5.662075 & 2.891767 \\
\hline $\mathrm{H}$ & -3.48 & 4.499400 & 3.22 \\
\hline & -4.616 & 1.579426 & 0.29 \\
\hline $\mathrm{H}$ & -6.75 & 2.724664 & -0.01 \\
\hline $\mathrm{H}$ & -12.0 & 4.050894 & 3.1 \\
\hline $\mathrm{H}$ & -14.2 & 3.18 & 2. \\
\hline $\mathrm{H}$ & -14.6 & 5.407249 & -0.8 \\
\hline $\mathrm{H}$ & $-12 \cdot 3$ & 6.273787 & -0.5 \\
\hline C & 7.7 & -4.820425 & 1.35 \\
\hline C & 8.442 & -4.849591 & 0.14 \\
\hline C & 9. & -5.0 & \\
\hline $\mathrm{C}$ & 10.519 & -5.315 & 1.30 \\
\hline $\mathrm{C}$ & 9. & -5.4 & \\
\hline $\mathrm{C}$ & 8.424 & -5.172675 & 2.51 \\
\hline C & 11. & -5.183 & \\
\hline $\mathrm{C}$ & 6.359 & -4.272443 & 1.426558 \\
\hline $\mathrm{C}$ & 5 . & -4.760314 & 2.3 \\
\hline C & 4.200515 & -4.103166 & 2.51 \\
\hline $\mathrm{C}$ & 3. & -2.936 & $1 . \varepsilon$ \\
\hline C & 4.818168 & -2.489954 & 0.855172 \\
\hline $\mathrm{C}$ & 6.026780 & -3.143178 & 0.67 \\
\hline $\mathrm{C}$ & 12.598551 & -4.396602 & 2.284256 \\
\hline $\mathrm{C}$ & 13.88 & -3.905392 & 2.11 \\
\hline $\mathrm{C}$ & 14.618 & -4.182453 & 0.96 \\
\hline $\mathrm{C}$ & 14.08 & -5.113260 & 0.0 \\
\hline$C$ & 12.79 & -5.602370 & 0.23 \\
\hline $\mathrm{H}$ & 7.92 & -4.629548 & $-0.7 \varepsilon$ \\
\hline $\mathrm{H}$ & 10.343 & -5.005703 & -0.815577 \\
\hline $\mathrm{H}$ & 10.30 & -5.641400 & 3.42 \\
\hline $\mathrm{H}$ & 7.902 & -5.173813 & 3.465985 \\
\hline $\mathrm{H}$ & 5.616 & -5.662075 & 2.89 \\
\hline I & 3.481 & -4.499400 & 3.221202 \\
\hline $\mathrm{H}$ & 4.61 & -1.579426 & 0.2 \\
\hline $\mathrm{H}$ & 6.754 & -2.724664 & -0.014537 \\
\hline $\mathrm{H}$ & 12.01 & -4.050894 & 3.12 \\
\hline . & 14.259 & -3.183341 & 2.835568 \\
\hline $\mathrm{H}$ & 14.65 & -5.407249 & -0.807050 \\
\hline 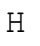 & 12.383 & -6.273787 & -0.516238 \\
\hline $\mathrm{C}$ & 7.736419 & 4.820425 & -1.350029 \\
\hline $\mathrm{C}$ & 8.442 & 4.849591 & -0.145434 \\
\hline $\mathrm{C}$ & 9.807 & 5.087378 & -0.124741 \\
\hline $\mathrm{C}$ & 10.519 & 5.315695 & $-1 \cdot 3$ \\
\hline
\end{tabular}




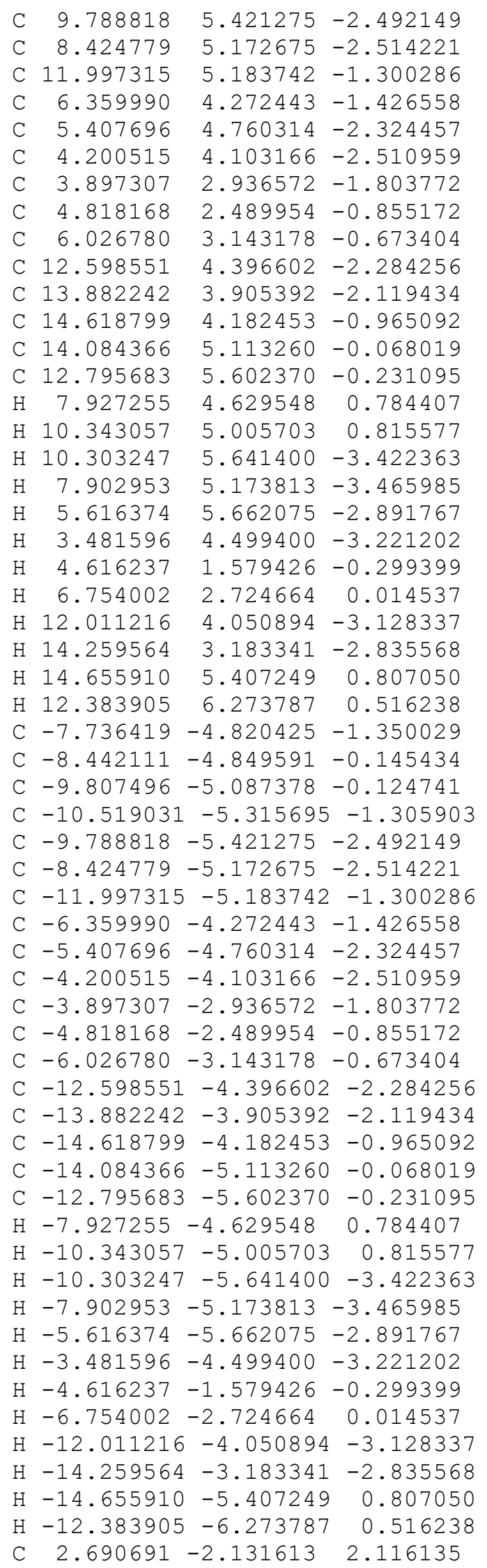

\begin{tabular}{|c|c|c|c|}
\hline & 10 & 850 & 713 \\
\hline & 0.882006 & -0.720046 & 1.478518 \\
\hline & 0.560285 & -0.458746 & 2.821210 \\
\hline & 1.310073 & -1.070137 & 3822082 \\
\hline & 2.359805 & -1.896493 & 3.464178 \\
\hline & -0.560285 & 0.458746 & 2.821210 \\
\hline & -0.882006 & 0.720046 & 1.478518 \\
\hline & -1.935210 & 1.541850 & 1.103713 \\
\hline & -2.690691 & 2.131613 & 16135 \\
\hline & -2.3 & & 3.464178 \\
\hline & 0073 & 1.070137 & 3.82208 \\
\hline & 0.000000 & 0.000000 & 0.677763 \\
\hline & 2.156170 & -1.722709 & 0.057116 \\
\hline & 8346 & 38330 & \\
\hline & 2.975008 & -2.341688 & 48 \\
\hline & -2.156170 & 1.722709 & 0. \\
\hline & -2.975008 & 2.341688 & $4.22 x-2 \cdot 3$ \\
\hline & -1.088346 & 0.888330 & 4.868565 \\
\hline & 2.690691 & 2.131613 & -2.116135 \\
\hline & 1.9 & & -1 \\
\hline & 0.8 & 46 & -1 \\
\hline & 0.5 & 0.4 & -2. \\
\hline & 1.3. & 1. & -3.82 \\
\hline C & 2.3 & 493 & -3. \\
\hline & -0.560285 & -0.458746 & -2.821210 \\
\hline & $-0 . \varepsilon$ & -0 & -1 \\
\hline & -1. & -1 & -1 \\
\hline & -2.690691 & -2.1 & -2 \\
\hline & -2.359805 & -1.896493 & -3.4 \\
\hline & -1.310073 & -1.070137 & -3.822082 \\
\hline & 0.0 & 000 & -0.67 \\
\hline & 2.1 & 09 & -0 . \\
\hline & 1.0 & 0. & -4 . \\
\hline $\mathrm{H}_{\mathrm{H}}$ & 2.97 & 2 . & -4 . \\
\hline $\mathrm{H}$ & -2.156170 & -1.722709 & -0.057116 \\
\hline $\mathrm{H}$ & -2.975008 & -2.341688 & -4.238448 \\
\hline & -1.0 & -0.88 & -4.8 \\
\hline & -15 & -3 & $3-0$ \\
\hline & -16.89 & -0.739102 & -0 \\
\hline $\mathrm{C}$ & 16.893064 & 0.739102 & -0.075987 \\
\hline C & 15.713855 & 3.245848 & -0.604024 \\
\hline C & -16.508195 & -2.609896 & -1.565318 \\
\hline & -16.306062 & -1.486789 & 0.9 \\
\hline & 16.3 & 1.48 & 0.94 \\
\hline & 16.5 & 2.6 & -1.5 \\
\hline & -15.725921 & -2.717458 & 0.688234 \\
\hline C & -17.087433 & $3-1.376193$ & $3-1.306407$ \\
\hline C & 17.0874 & 1.376193 & -1.306407 \\
\hline C & 15.7 & 2.717458 & 0.68823 \\
\hline & -16.598128 & -3.039091 & -2.55 \\
\hline & -17.615445 & -0.860146 & -21 \\
\hline & -15.134415 & -3.187369 & 1.466842 \\
\hline 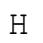 & -16.147613 & $3-1.032212$ & 1.919860 \\
\hline $\mathrm{H}$ & 16.598128 & 3.039091 & -2.55855 \\
\hline & & 0.860146 & -2.1025 \\
\hline & & 3.1873 & 1.4668 \\
\hline & 16 & 1.0322 & 1.9198 \\
\hline
\end{tabular}


$\begin{array}{llcc}\text { C } & -15.713855 & 3.245848 & 0.604024 \\ \text { C } & 15.713855 & -3.245848 & 0.604024 \\ \text { C } & -16.893064 & 0.739102 & 0.075987 \\ \text { C } & 16.893064 & -0.739102 & 0.075987 \\ \text { C } & -15.725921 & 2.717458 & -0.688234 \\ \text { C } & 15.725921 & -2.717458 & -0.688234 \\ \text { C } & -16.508195 & 2.609896 & 1.565318 \\ \text { C } & 16.508195 & -2.609896 & 1.565318 \\ \text { C } & -17.087433 & 1.376193 & 1.306407 \\ \text { C } & 17.087433 & -1.376193 & 1.306407 \\ \text { C } & -16.306062 & 1.486789 & -0.947704 \\ \text { C } & 16.306062 & -1.486789 & -0.947704 \\ \mathrm{H} & -15.134415 & 3.187369 & -1.466842 \\ \mathrm{H} & -16.147613 & 1.032212 & -1.919860 \\ \mathrm{H} & 16.147613 & -1.032212 & -1.919860 \\ \mathrm{H} & 15.134415 & -3.187369 & -1.466842 \\ \mathrm{H} & 17.615445 & -0.860146 & 2.102586 \\ \mathrm{H} & 16.598128 & -3.039091 & 2.558554 \\ \mathrm{H} & -16.598128 & 3.039091 & 2.558554 \\ \mathrm{H} & -17.615445 & 0.860146 & 2.102586\end{array}$ $\Delta \mathrm{H}=-4725.715819$

\section{2-4}

$\begin{array}{lrrr}\text { C } & 8.778030 & 2.350137 & -1.439601 \\ \mathrm{C} & 9.314045 & 2.613529 & -0.176246 \\ \mathrm{C} & 10.282291 & 1.778902 & 0.354957 \\ \mathrm{C} & 10.730274 & 0.658180 & -0.361026 \\ \mathrm{C} & 10.515163 & 0.697878 & -1.746709 \\ \mathrm{C} & 9.529249 & 1.519147 & -2.276706 \\ \mathrm{C} & 7.314654 & 2.472499 & -1.668137 \\ \mathrm{C} & 6.688443 & 2.783429 & -2.874731 \\ \mathrm{C} & 5.343021 & 2.494245 & -3.072131 \\ \mathrm{C} & 4.571126 & 1.858672 & -2.089443 \\ \mathrm{C} & 5.180579 & 1.647614 & -0.850649 \\ \mathrm{C} & 6.513036 & 1.950242 & -0.644934 \\ \mathrm{H} & 8.838616 & 3.345304 & 0.469428 \\ \mathrm{H} & 10.508284 & 1.882591 & 1.409606 \\ \mathrm{H} & 10.943550 & -0.061386 & -2.391837 \\ \mathrm{H} & 9.210352 & 1.355366 & -3.301742 \\ \mathrm{H} & 7.262202 & 3.226237 & -3.683278 \\ \mathrm{H} & 4.900982 & 2.752040 & -4.028157 \\ \mathrm{H} & 4.642075 & 1.169196 & -0.041066 \\ \mathrm{H} & 6.968778 & 1.671809 & 0.298825 \\ \mathrm{C} & -8.778030 & -2.350137 & -1.439601 \\ \mathrm{C} & -9.314045 & -2.613529 & -0.176246 \\ \mathrm{C} & -10.282291 & -1.778902 & 0.354957 \\ \mathrm{C} & -10.730274 & -0.658180 & -0.361026 \\ \mathrm{C} & -10.515163 & -0.697878 & -1.746709 \\ \mathrm{C} & -9.529249 & -1.519147 & -2.276706 \\ \mathrm{C} & -1.314654 & -2.472499 & -1.668137 \\ \mathrm{C} & -6.688443 & -2.783429 & -2.874731 \\ \mathrm{C} & -5.343021 & -2.494245 & -3.072131 \\ \mathrm{C} & -4.571126 & -1.858672 & -2.089443 \\ \mathrm{C} & -5.180579 & -1.647614 & -0.850649 \\ \mathrm{C} & -6.513036 & -1.950242 & -0.644934\end{array}$

\begin{tabular}{|c|c|c|c|}
\hline $\mathrm{H}$ & -8.838616 & -3.345304 & 0.469428 \\
\hline $\mathrm{H}$ & -10.508284 & -1.882591 & 1.409606 \\
\hline $\mathrm{H}$ & -10.943550 & 0.061386 & -2.391837 \\
\hline $\mathrm{H}$ & -9.210352 & -1.355366 & -3.301742 \\
\hline 11 & -7.262202 & -3.226237 & -3.683278 \\
\hline 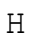 & -4.900982 & -2.752040 & -4.028157 \\
\hline $\mathrm{H}$ & -4.642075 & -1.169196 & -0.041066 \\
\hline $\mathrm{H}$ & -6.968778 & -1.671809 & 0.298825 \\
\hline C & -8.778030 & 2.350137 & 1.43 \\
\hline 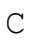 & -9.314045 & 2.613529 & 0.176246 \\
\hline 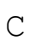 & -10.282291 & 1.778902 & -0.3 \\
\hline 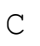 & -10.730274 & 0.658180 & 0.3 \\
\hline 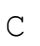 & -10.5 & 0.697878 & 1. \\
\hline C & -9.529249 & 1.519147 & 2.276706 \\
\hline $\mathrm{C}$ & -7.314 & 2.472499 & 1.6 \\
\hline $\mathrm{C}$ & -6.688443 & 2.783429 & 2.87 \\
\hline $\mathrm{C}$ & -5.34 & 2.494245 & 3.07 \\
\hline $\mathrm{C}$ & -4.571126 & 1.858672 & 2.08 \\
\hline $\mathrm{C}$ & -5.18 & 1.647 & $0 . \varepsilon$ \\
\hline $\mathrm{C}$ & -6.513036 & 1.950242 & 0.64 \\
\hline $\mathrm{H}$ & -8.83 & 3.34 & -0.46 \\
\hline $\mathrm{H}$ & -10.508284 & 1.882591 & -1.4 \\
\hline $\mathrm{H}$ & -10.9 & -0.0 & \\
\hline $\mathrm{H}$ & -9.210352 & 1.355366 & 3. \\
\hline $\mathrm{H}$ & -7.2622 & 3.2262 & 3.6 \\
\hline $\mathrm{H}$ & -4 & 2.75 & 4. \\
\hline $\mathrm{H}$ & -4.642075 & 1.16 & 0.0 \\
\hline $\mathrm{H}$ & -6 . & $1.6^{7}$ & -0.2 \\
\hline $\mathrm{C}$ & 8.778030 & -2.35 & 1.4 \\
\hline $\mathrm{C}$ & 9 . & -2.6 & 0. \\
\hline $\mathrm{C}$ & 10.282291 & -1.778902 & -0.3 \\
\hline $\mathrm{C}$ & 10. & -0.6 & 0 \\
\hline $\mathrm{C}$ & 10.515 & -0.69 & 1.7 \\
\hline $\mathrm{C}$ & 9. & -1.5 & 2.2 \\
\hline $\mathrm{C}$ & 7.314654 & -2.472499 & 1.66 \\
\hline $\mathrm{C}$ & 6.688443 & -2.78 & 2.8 \\
\hline $\mathrm{C}$ & 5.343021 & -2.494245 & 3.072 \\
\hline $\mathrm{C}$ & 4.57 & -1.85 & 2.0 \\
\hline $\mathrm{C}$ & 5.180 & -1.647614 & 0.850 \\
\hline $\mathrm{C}$ & 6.513 & -1.950242 & 0.6 \\
\hline $\mathrm{H}$ & 8.838 & -3.34 & -0.46 \\
\hline $\mathrm{H}$ & 10.50 & -1.882591 & -1.4 \\
\hline $\mathrm{H}$ & 10.94 & 0.061386 & 2.391 \\
\hline $\mathrm{H}$ & 9.21 & -1.35 & 3.30 \\
\hline $\mathrm{H}$ & 7.262202 & -3.226237 & 3.6832 \\
\hline $\mathrm{H}$ & 4.90 & -2.7 & 4.02 \\
\hline $\mathrm{H}$ & 4.642 & -1.169196 & 0.0410 \\
\hline $\mathrm{H}$ & 6.96 & $-1 \cdot 6$ & -0.29 \\
\hline $\mathrm{C}$ & -3.206340 & -1.327926 & -2.36100 \\
\hline $\mathrm{C}$ & $-2 \cdot 34$ & -0.9 & -1.31 \\
\hline $\mathrm{C}$ & -1.122 & -0.357179 & -1.58387 \\
\hline $\mathrm{C}$ & -0.68 & -0.2 & -2.916039 \\
\hline $\mathrm{C}$ & -1.517 & -0.608519 & -3.9619 \\
\hline $\mathrm{C}$ & -2.775777 & -1.118162 & -3.67865 \\
\hline $\mathrm{C}$ & 0.688 & 0.2 & -2.9160 \\
\hline $\mathrm{C}$ & 1 . & 7179 & -1.583878 \\
\hline $\mathrm{C}$ & 2.348663 & 0.955563 & -1.31539 \\
\hline
\end{tabular}




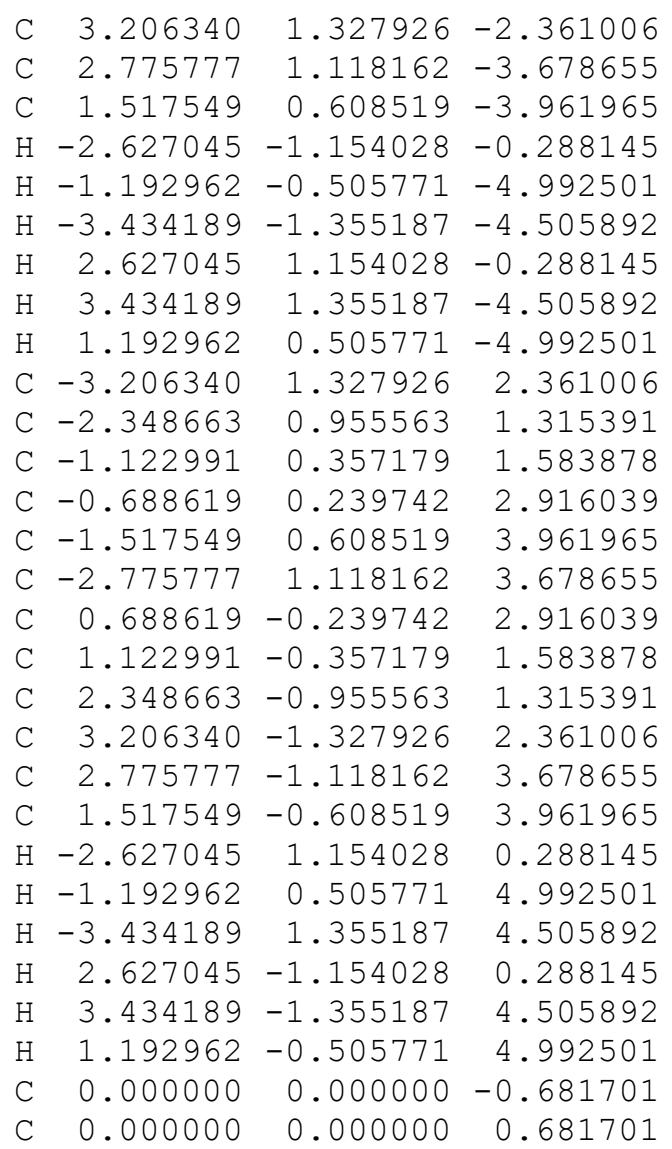

$\Delta \mathrm{H}=-2844.851667$

\section{$2-5$}

3.1347331 .571191

$\begin{array}{llll}\text { C }-10.473635 & 2.998897 & 0.036266\end{array}$

$\begin{array}{llll}\text { C }-11.269003 & 2.361054 & 0.998786\end{array}$

$\begin{array}{llll}\text { C }-10.799383 & 2.405694 & 2.317961\end{array}$

C $-9.489820 \quad 2.772213 \quad 2.597264$

C $-7.150877 \quad 2.941400 \quad 1.721021$

C $-6.411135 \quad 3.321792 \quad 2.840813$

$\begin{array}{llll}\text { C }-5.105991 & 2.880573 & 3.014807\end{array}$

C $-4.491588 \quad 2.026162 \quad 2.090772$

$\begin{array}{llll}\text { C }-5.209806 & 1.715232 & 0.934695\end{array}$

C $-6.506857 \quad 2.161856 \quad 0.753856$

$\mathrm{H}-8.546199 \quad 3.750262-0.488878$

$\mathrm{H}-10.804439 \quad 3.062355-0.993090$

$\mathrm{H}-11.401953 \quad 2.008929 \quad 3.127702$

$\mathrm{H}-9.108552 \quad 2.630573 \quad 3.604044$

$\mathrm{H}-6.863148 \quad 3.961507 \quad 3.592827$

$\mathrm{H}-4.560532 \quad 3.207786 \quad 3.893508$

$\mathrm{H}-4.778035 \quad 1.067230 \quad 0.180404$

$\mathrm{H}-7.059149 \quad 1.829748-0.118845$

C $8.613805 \quad-3.134733 \quad 1.571191$

$\begin{array}{llll}\text { C } \quad 9.172672 & -3.377251 & 0.315257\end{array}$

$\begin{array}{llll}\text { C } 10.473635 & -2.998897 & 0.036266\end{array}$
C $11.269003-2.361054$

C $10.799383-2.405694$

C $9.489820-2.772213$

C $7.150877-2.941400$

C $6.411135-3.321792$

C $5.105991-2.880573$

C $4.491588-2.026162$

C $5.209806-1.715232$

C $6.506857-2.161856$

$\mathrm{H} \quad 8.546199-3.750262$

H $10.804439-3.062355$

H $11.401953-2.008929$

$\mathrm{H} \quad 9.108552-2.630573$

$\mathrm{H} \quad 6.863148-3.961507$

$\mathrm{H} \quad 4.560532-3.207786$

$\mathrm{H} \quad 4.778035-1.067230$

H $7.059149-1.829748-0.118845$

C $8.613805 \quad 3.134733-1.571191$

C $\quad 9.172672 \quad 3.377251-0.315257$

C $10.473635 \quad 2.998897-0.036266$

C $11.269003 \quad 2.361054-0.998786$

C $10.799383 \quad 2.405694-2.317961$

C $9.489820 \quad 2.772213-2.597264$

C $12.213237 \quad 1.297229-0.542837$

C $7.150877 \quad 2.941400-1.721021$

C $6.411135 \quad 3.321792-2.840813$

$\begin{array}{llll}\text { C } \quad 5.105991 & 2.880573 & -3.014807\end{array}$

C $4.491588 \quad 2.026162-2.090772$

C $5.209806 \quad 1.715232-0.934695$

C $6.5068572 .161856-0.753856$

C $12.433288 \quad 0.178902-1.358445$

C $12.454461-1.097981-0.822940$

C $12.213237-1.297229 \quad 0.542837$

C $12.433288-0.178902-1.358445$

$\begin{array}{llll}\text { C } 12.454461 & 1.097981 & 0.822940\end{array}$

$\begin{array}{llll}\mathrm{H} & 8.546199 & 3.750262 & 0.488878\end{array}$

$\mathrm{H} 10.804439 \quad 3.062355 \quad 0.993090$

H $11.4019532 .008929-3.127702$

$\mathrm{H} \quad 9.108552 \quad 2.630573-3.604044$

H $\quad 6.863148 \quad 3.961507 \quad-3.592827$

$\mathrm{H} \quad 4.560532 \quad 3.207786-3.893508$

$\mathrm{H} \quad 4.778035 \quad 1.067230-0.180404$

$\begin{array}{llll}\mathrm{H} & 7.059149 & 1.829748 & 0.118845\end{array}$

$\mathrm{H} \quad 12.395020 \quad 0.268276-2.438083$

H $12.463985-1.936356-1.510419$

H $12.395020-0.268276 \quad 2.438083$

H $12.463985 \quad 1.936356 \quad 1.510419$

C $-8.613805-3.134733-1.571191$

C $-9.172672-3.377251-0.315257$

C $-10.473635-2.998897-0.036266$

C $-11.269003-2.361054-0.998786$

C $-10.799383-2.405694-2.317961$

C $-9.489820-2.772213-2.597264$

C $-12.213237-1.297229-0.542837$

C $-7.150877-2.941400-1.721021$

C $-6.411135-3.321792-2.840813$

C $-5.105991-2.880573-3.014807$ 


\begin{tabular}{|c|c|c|c|}
\hline C & -4.491588 & -2.026162 & -2.090772 \\
\hline C & -5.209806 & -1.715232 & -0.934695 \\
\hline 乙 & -6.506857 & -2.161856 & -0.753856 \\
\hline & -12.433288 & -0.178902 & -1.358445 \\
\hline $\mathcal{C}$ & -12.454461 & 1.097981 & -0.822940 \\
\hline C & -12.213237 & 1.297229 & 0.542837 \\
\hline C & -12.433288 & 0.178902 & 1.3 \\
\hline & -12.454461 & -1.097981 & 940 \\
\hline & -8.546199 & -3.750262 & 0.488878 \\
\hline & -10.804439 & -3.062355 & 090 \\
\hline & -11.401953 & -2.008929 & -3 \\
\hline & -9.108 & -2.630573 & -3.604 \\
\hline & -6.863 & -3.961507 & -3.592 \\
\hline & -4.560532 & -3.207786 & -3.893508 \\
\hline & -4.778035 & -1.067230 & -0.180404 \\
\hline & -7.059149 & -1.829748 & 0.118845 \\
\hline & -12.395020 & -0.268276 & $5-2.438083$ \\
\hline 1 & -12.463985 & 1.936356 & $5-1.510419$ \\
\hline & -12.395020 & 0.268276 & 2.438083 \\
\hline 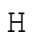 & -12.463985 & -1.936356 & 1.510419 \\
\hline & 3.164672 & -1.409607 & 2.352493 \\
\hline 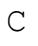 & 2.332919 & -0.991668 & 1.305392 \\
\hline$\checkmark$ & 1.119683 & -0.368527 & 1.579144 \\
\hline C & 0.684582 & -0.252343 & 2.911412 \\
\hline C & 1.500429 & -0.648810 & 3.95 \\
\hline$C$ & 2.740992 & -1.198392 & 3.672173 \\
\hline C & -0.684582 & 0.252343 & 2.911412 \\
\hline C & -1.119683 & 0.368527 & 1.579144 \\
\hline $\mathrm{C}$ & -2.332919 & 0.991668 & 1.305392 \\
\hline C & -3.164672 & 1.409607 & 2.352493 \\
\hline C & -2.740992 & 1.198392 & 3.672173 \\
\hline $\mathrm{C}$ & -1.500429 & 0.648810 & 3.957781 \\
\hline $\mathrm{H}$ & 2.617950 & -1.186894 & 0.277949 \\
\hline $\mathrm{H}$ & 1.175960 & -0.543 & 4.98 \\
\hline $\mathrm{H}$ & 3.394314 & -1.469686 & 4.493679 \\
\hline $\mathrm{H}$ & -2.617950 & 1.186894 & 0.27 \\
\hline $\mathrm{H}$ & -3.394314 & 1.469686 & 4.493679 \\
\hline $\mathrm{H}$ & -1 & 0.543764 & 4.988134 \\
\hline $\mathrm{C}$ & 3.164672 & 1.409607 & -2.352493 \\
\hline C & 2.332 & 0.991668 & -1.305392 \\
\hline $\mathrm{C}$ & 1.119683 & 0.368527 & -1.579144 \\
\hline $\mathrm{C}$ & 0.684582 & 0.252343 & -2.911412 \\
\hline $\mathrm{C}$ & 1.500429 & 0.648810 & -3.957781 \\
\hline $\mathrm{C}$ & 2.740992 & 1.198392 & -3.672173 \\
\hline $\mathrm{C}$ & -0.684582 & -0.252343 & -2.911412 \\
\hline C & -1.119683 & -0.368527 & -1.579144 \\
\hline $\mathrm{C}$ & -2.332919 & -0.991668 & -1.305392 \\
\hline $\mathrm{C}$ & -3.164672 & -1.409607 & -2.352493 \\
\hline $\mathrm{C}$ & -2.740992 & -1.198392 & -3.672173 \\
\hline $\mathrm{C}$ & -1.500429 & -0.648810 & -3.957781 \\
\hline 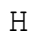 & 2.617950 & 1.186894 & -0.277949 \\
\hline $\mathrm{H}$ & 1.175960 & 0.543764 & -4.988134 \\
\hline $\mathrm{H}$ & 3.394314 & 1.469686 & -4.493679 \\
\hline 11 & -2.617950 & -1.186894 & -0.277949 \\
\hline $\mathrm{H}$ & -3.394314 & -1.469686 & -4.493679 \\
\hline 1 & -1.175960 & -0.543764 & -4.988134 \\
\hline 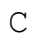 & 0.000000 & 0.000000 & 0.680936 \\
\hline
\end{tabular}

C $\quad 0.000000 \quad 0.000000-0.680936$ $\Delta \mathrm{H}=-3306.745195$

\begin{tabular}{|c|c|c|c|}
\hline \multicolumn{4}{|c|}{-6} \\
\hline 0 & -8.473138 & 3.575543 & 1.763062 \\
\hline & -9.132602 & 3.706016 & 0.539824 \\
\hline & -10.513208 & 3.623353 & 0.466632 \\
\hline$U$ & -11.287844 & 3.417161 & 1.613485 \\
\hline & -10.639513 & 3.495075 & 2.849443 \\
\hline C & -9.255405 & 3.564779 & 2.921758 \\
\hline & -12.602784 & 2.746455 & 1.437795 \\
\hline C & -7.032890 & 3.225362 & 1.820838 \\
\hline & -6.175016 & 3.679802 & 2.823722 \\
\hline C & -4.897804 & 3.155853 & 2.960311 \\
\hline 0 & -4.427734 & 2.150614 & 2.107649 \\
\hline $\mathrm{C}$ & -5.259102 & 1.756349 & 1.05 \\
\hline 0 & -6.534242 & 2.279235 & 0.919746 \\
\hline 0 & -12.833096 & 1.594696 & 33122 \\
\hline$U$ & -13.587827 & 0.552761 & 1.682014 \\
\hline $\mathrm{C}$ & -14.140594 & 0.628319 & 0332 \\
\hline C & -14.1 & 1.897396 & $6-0$ \\
\hline 0 & -13 & 2.93 & 556 \\
\hline 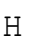 & -8.555824 & 3.783009 & -0.376427 \\
\hline $\mathrm{H}$ & -10.983426 & 3.586745 & $5-0.510319$ \\
\hline $\mathrm{H}$ & -11.212572 & 3.397039 & 6302 \\
\hline $\mathrm{H}$ & -8.773718 & 3.499020 & 504 \\
\hline $\mathrm{H}$ & -6.512248 & 4.449052 & 3.51 \\
\hline $\mathrm{H}$ & -4.254541 & 3.537 & 3.7 \\
\hline $\mathrm{H}$ & -4.928836 & 0.987859 & 0.368885 \\
\hline $\mathrm{H}$ & -7.179864 & 1.890425 & 0.13 \\
\hline H & -12.257448 & 1.417059 & 5045 \\
\hline $\mathrm{H}$ & -13.562158 & -0.396684 & 4622 \\
\hline $\mathrm{H}$ & -14.697866 & 2.039567 & $7-1.1$ \\
\hline $\mathrm{H}$ & -13.352374 & 3.867981 & $1-0.24$ \\
\hline $\mathrm{C}$ & 8.473138 & -3.575543 & 1.763062 \\
\hline C & 9.132602 & -3.706016 & 0.53 \\
\hline $\mathrm{C}$ & 10.513208 & -3.623353 & 0.466632 \\
\hline C & 11.28 & -3.417161 & 1.61 \\
\hline C & 10.639513 & -3.495075 & 2.84 \\
\hline $\mathrm{C}$ & 9.255405 & -3.564779 & 2.92 \\
\hline $\mathrm{C}$ & 12.602784 & -2.746455 & 1.4 \\
\hline $\mathrm{C}$ & 7.032890 & -3.225362 & 1.82 \\
\hline$C^{\prime}$ & 6.175016 & -3.679802 & 2.823722 \\
\hline $\mathrm{C}$ & 4.897804 & -3.155853 & 2.960311 \\
\hline C & 4.427734 & -2.150614 & 2.107649 \\
\hline C & 5.259102 & -1.756349 & 1.059058 \\
\hline $\mathrm{C}$ & 6.534242 & -2.279235 & 0.919746 \\
\hline C & 12.833096 & -1.594696 & 2.193122 \\
\hline C & 13.587827 & -0.552761 & 1.682014 \\
\hline c & 14.140594 & -0.628319 & 0.400332 \\
\hline C & 14.178426 & -1.897396 & -0.194401 \\
\hline C & 13.412992 & -2.937886 & 0.311556 \\
\hline $\mathrm{H}$ & 8.555824 & -3.783009 & -0.376427 \\
\hline $\mathrm{H}$ & 10.983426 & -3.586745 & -0.510319 \\
\hline $\mathrm{H}$ & 11.212572 & -3.397039 & 3.766302 \\
\hline
\end{tabular}




\begin{tabular}{|c|c|c|c|}
\hline & 718 & -3.499020 & 2504 \\
\hline & 6.512248 & -4.449052 & 3.511760 \\
\hline & 4.254541 & -3.537117 & 3.746603 \\
\hline & 4.928836 & -0.987859 & 0.368885 \\
\hline & 7.179864 & -1.890425 & 0.139136 \\
\hline & 12.257448 & -1.417059 & 3.095045 \\
\hline & 13.562158 & 0.396684 & 2.204622 \\
\hline & 97866 & -2.039567 & -1.137052 \\
\hline & 13.352374 & -3.867981 & 5498 \\
\hline & 8.473138 & 3.575543 & -1.763062 \\
\hline & 9.132602 & 3.706016 & -0.539824 \\
\hline & 10.513208 & 3.623353 & -0.466632 \\
\hline & 11.287844 & 3.417161 & -1.613485 \\
\hline & 10.639513 & 3.495075 & -2.849443 \\
\hline & 9.255405 & 3.564779 & -2.921758 \\
\hline & 12.602784 & 2.746455 & -1.437795 \\
\hline & 7.032890 & 3.225362 & -1.820838 \\
\hline & 6.175016 & 3.679802 & -2.823722 \\
\hline & 4.897804 & 3.155853 & -2.960311 \\
\hline & 4.427734 & 2.150614 & -2.107649 \\
\hline & 5.259102 & 1.756349 & -1.059058 \\
\hline & 6.534242 & 2.279235 & -0.919746 \\
\hline & 12.833096 & 1.594696 & -2.193122 \\
\hline & 13.587827 & 0.552761 & -1.682014 \\
\hline & 14.140594 & 0.628319 & -0.400332 \\
\hline & 14.178426 & 1.897396 & 0.194401 \\
\hline & 13.412992 & 2.937886 & -0.311556 \\
\hline & 8.555824 & 3.783009 & 0.376427 \\
\hline & 10.983426 & 3.586745 & 0.510319 \\
\hline & 11.212572 & 3.397039 & -3.766302 \\
\hline & 8.773718 & 3.499020 & -3.892504 \\
\hline & 6.512248 & 052 & 1760 \\
\hline & 4.254541 & 3.537117 & -3.746603 \\
\hline & 4.928836 & 0.987859 & -0.368885 \\
\hline & 7.179864 & 1.890425 & -0.139136 \\
\hline & 12.257448 & 1.417059 & -3.095045 \\
\hline & 13.5 & -0.396684 & -2.204622 \\
\hline & 14.6 & 2.039567 & 7052 \\
\hline & 13.352374 & 3.867981 & 0.245498 \\
\hline & -8.473138 & -3.575543 & -1.763062 \\
\hline & -9.132602 & -3.706016 & -0.53982 \\
\hline & -10.513208 & $3-3.623353$ & $3-0.466632$ \\
\hline & -11.287844 & $4-3.417161$ & -1.613485 \\
\hline & -10.639513 & $3-3.495075$ & -2.849443 \\
\hline & -9.255405 & -3 & -2 \\
\hline & -12.602 & 16 & $37^{\circ}-x \cdot$ \\
\hline & -7.032890 & -3.225362 & -1.820838 \\
\hline & -6.175016 & -3.679802 & -2.823722 \\
\hline & -4.897804 & -3.155853 & -2.960311 \\
\hline & -4.427734 & -2.150614 & -2.107649 \\
\hline & -5.259102 & -1.756349 & -1.059058 \\
\hline & -6.534242 & -2.279235 & -0.919746 \\
\hline & -12.83309 & -1.594696 & -2.193122 \\
\hline & -13.587827 & $7-0.552761$ & -1.682014 \\
\hline & -14.140594 & $4-0.628319$ & -0.400332 \\
\hline & -14.178426 & $5-1.8973$ & 0.19444 \\
\hline & -13.4129 & $2-$ & \\
\hline
\end{tabular}

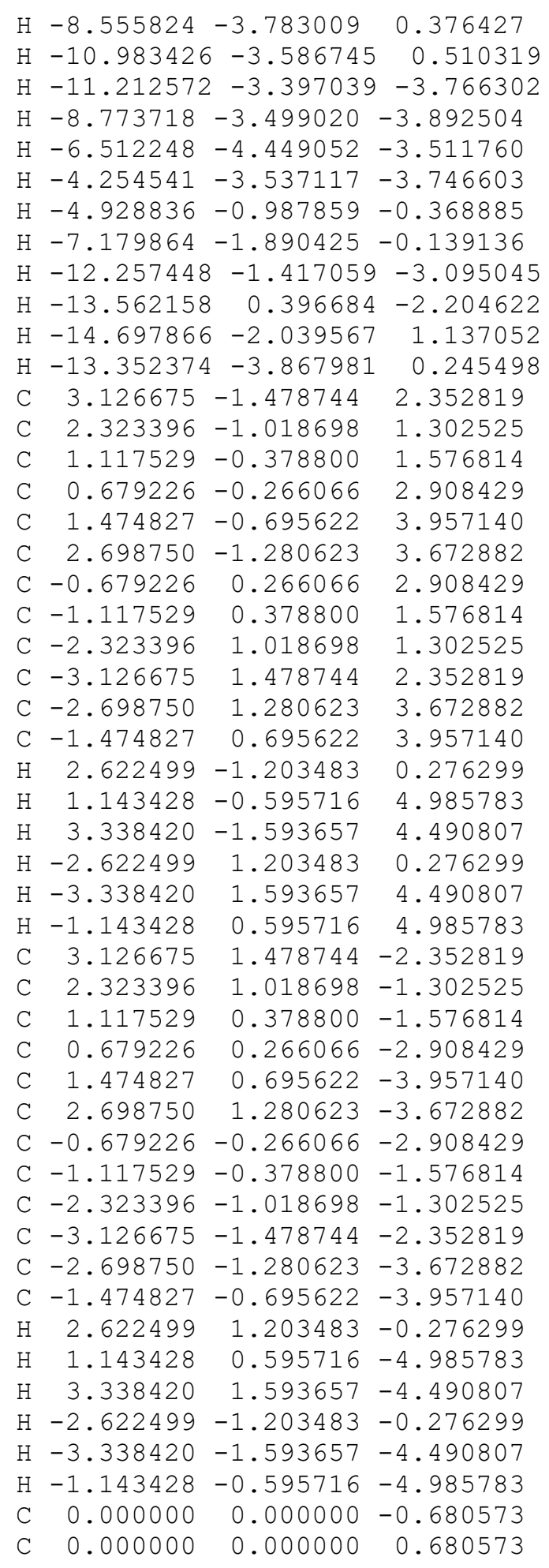

$\Delta \mathrm{H}=-3768.640348$ 


\section{2-7}

\begin{tabular}{|c|c|c|c|}
\hline & -8.361929 & 3.853842 & 981 \\
\hline & -9.079859 & 3.937010 & 0.641675 \\
\hline & -10.458924 & 4.074773 & 0.648812 \\
\hline & -11.172473 & 42084 & 9409 \\
\hline & -10.438719 & 4.208095 & 5457 \\
\hline & -9.059615 & 4.061741 & 3.0 \\
\hline & -12.624355 & 3.835681 & 1.829121 \\
\hline & -6.952813 & 3.391386 & 1.8 \\
\hline & -6.020243 & 3.884294 & 2.774900 \\
\hline & -4.767375 & 3.303873 & 2.898547 \\
\hline & -4.395886 & 2.206312 & 2.115163 \\
\hline & -5.301866 & 1.761790 & 1.15293 \\
\hline & -6.5 & 2.339164 & 1.0294 \\
\hline & -13.102629 & 2.867257 & 2.712984 \\
\hline & -14.256374 & 2.156288 & 2. \\
\hline & -14.987242 & 2.380242 & 1.263597 \\
\hline & -14.640720 & 3.518905 & 0.522777 \\
\hline & -13.477447 & 4.225404 & 0.792395 \\
\hline & -8.560944 & 3.831488 & -0.30 \\
\hline & -10.998514 & 4.027371 & -0.291458 \\
\hline & -10.957783 & 4.304057 & 3.985105 \\
\hline & -8.527598 & 4.021787 & 3.974767 \\
\hline & -6.278861 & 4.732469 & 3.401226 \\
\hline & -4.062321 & 3.712433 & 3.61534 \\
\hline & -5.0 & 0.913367 & 0.52 \\
\hline & -7.262697 & 1.920347 & 0.320218 \\
\hline & -12.486885 & 2.550320 & 3.548077 \\
\hline & -14.488138 & 1.313025 & 3.073886 \\
\hline & -15.224855 & 3.806015 & -0.344855 \\
\hline & -13.187317 & 5.037209 & 2274 \\
\hline & 8 & -3.853842 & 1.835981 \\
\hline & 9.079859 & -3.937010 & 0.641675 \\
\hline & 10.458924 & -4.074773 & 0.648812 \\
\hline & 11.172473 & -4.142084 & 1.849409 \\
\hline & 10.438719 & -4.208095 & $3.03645^{\circ}$ \\
\hline & 615 & -4.061741 & 3.029424 \\
\hline & 12.624355 & -3.835681 & 1.8 \\
\hline & 6.952813 & -3.391386 & 1.860006 \\
\hline & 6.020243 & -3.884294 & 2.774900 \\
\hline & 4.767375 & -3.303873 & 2.898547 \\
\hline & 4.395886 & -2.206312 & 2.115163 \\
\hline & 5.3 & -1.761790 & 1.152935 \\
\hline & 119 & -2.339164 & 1.029445 \\
\hline & 13.102629 & -2.867257 & 2.712984 \\
\hline & 14.256374 & -2.156288 & 2.435425 \\
\hline & 14.987242 & -2.380242 & 1.263597 \\
\hline & 14.640720 & -3.518905 & 0.522777 \\
\hline & 17447 & -4.225404 & 0.792395 \\
\hline & 8.560944 & -3.831488 & -0.305823 \\
\hline & 10.998514 & -4.027371 & -0.291458 \\
\hline & 10.957783 & -4.304057 & 3.985105 \\
\hline & 8.527598 & -4.021787 & 3.974767 \\
\hline & 6.278861 & -4.732469 & 3.40122 \\
\hline & 62321 & -3.712433 & \\
\hline & & -0.9133 & \\
\hline
\end{tabular}

\begin{tabular}{|c|c|c|c|}
\hline & 597 & 0347 & 218 \\
\hline & 12.486885 & -2.550320 & 3.548077 \\
\hline & 14.488138 & -1.313025 & 3.073886 \\
\hline & 15.224855 & -3.806015 & -0.34 \\
\hline & 13.187317 & -5.037209 & 0.132274 \\
\hline & 8.361929 & 3.853842 & -1.835981 \\
\hline & 9.079859 & 3.937010 & -0.641675 \\
\hline & 10.458924 & 4.074773 & -0.648812 \\
\hline & 11.172473 & 4.142084 & -1.849409 \\
\hline & 10.438719 & 4.208095 & -3.036457 \\
\hline & 9.059615 & 4.061741 & -3.029424 \\
\hline & 12.624355 & 3.835681 & -1.829121 \\
\hline & 6.95281 & 3.391386 & -1.860006 \\
\hline & 6.020243 & 3.884294 & -2.774900 \\
\hline & 4.767375 & 3.303873 & -2.898547 \\
\hline & 4.395886 & 2.206312 & -2.1 \\
\hline & 5.301866 & 1.761790 & -1.152935 \\
\hline & 6.55611 & 2.339164 & -1.02944 \\
\hline & 13.102629 & 2.867257 & -2.712984 \\
\hline & 14.2 & 288 & -2 . \\
\hline & 14.9 & 42 & -1 \\
\hline & 14.640720 & 905 & -0 \\
\hline & 13.477447 & 404 & -0 \\
\hline & 8.5 & & 0. \\
\hline & 10.998514 & 4.027371 & 0.29145 \\
\hline & 10.957783 & 4.304057 & -3.5 \\
\hline & 8.52 & 87 & -3 \\
\hline & 6.278861 & 4.732469 & -3.401226 \\
\hline & 4.062321 & 2433 & -3.615343 \\
\hline & 5.044048 & 0.913367 & -0.528311 \\
\hline & 7.2 & 347 & -0 . \\
\hline & 12.4 & 320 & -3 \\
\hline & 14.488138 & 1.313025 & -3 \\
\hline & 15.2248 & 3.806015 & 0.3 \\
\hline & 13.187317 & 5.037209 & -0.132274 \\
\hline$C$ & -8.361929 & -3.853842 & -1.835981 \\
\hline & -9.07 & -3.9 & -0.6416 \\
\hline & -10 & -4 & $3-0$ \\
\hline & -11.172473 & -4.142084 & -1 \\
\hline 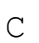 & -10.438719 & -4.208095 & $5-3.036457$ \\
\hline 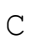 & -9.05961 & -4.0617 & -3.029424 \\
\hline$C$ & -12.624355 & -3.835 & -1.829121 \\
\hline$C$ & -6.952813 & -3.391386 & -1.860006 \\
\hline & -6.02 & -3.8 & -2.7 \\
\hline & -4.767375 & -3.303873 & -2.8 \\
\hline$C$ & -4.395886 & -2.206312 & -2.115163 \\
\hline$C$ & -5.301866 & -1.761790 & -1.15293 \\
\hline$C$ & -6.556119 & -2.339164 & -1.02944 \\
\hline $\mathrm{C}$ & -13.102629 & -2.867257 & -2.712984 \\
\hline & -14.256374 & -2.156 & -2 \\
\hline & -14.987242 & -2.380 & -1.26 \\
\hline & -14.640720 & -3.518905 & -0.52277 \\
\hline & -13.477447 & -4.225404 & -0.7923 \\
\hline- & & -3.83148 & 0.305823 \\
\hline $\mathrm{H}$ & -10.998514 & -4.02 & 0 . \\
\hline & -10.957783 & -4.30 & -3.9851 \\
\hline & -8.527 & -4.02178 & \\
\hline
\end{tabular}




\begin{tabular}{|c|c|c|c|}
\hline & & & \\
\hline & & -3.712433 & -3.6 \\
\hline & -5 & -0.913367 & -0.528311 \\
\hline & -7.26 & -1.92 & -0.320218 \\
\hline & -12.486885 & $5-2.550320$ & -3.548077 \\
\hline & -14.488138 & $3-1.313025$ & -3.073886 \\
\hline & -15.224855 & $5-3.806015$ & 0.344855 \\
\hline & -13.187317 & 37209 & 2274 \\
\hline & & -1.503963 & 2.35355 \\
\hline & 2.322610 & -1.023520 & 1.30205 \\
\hline & 1.117584 & -0.380361 & 1.576000 \\
\hline & 0.677068 & -0.271409 & 2.907139 \\
\hline & 1.461721 & -0.717738 & 3.957117 \\
\hline & 2.677976 & -1.318283 & \\
\hline & -0.677068 & 0.271409 & 2.907139 \\
\hline & -1.117584 & 0.380361 & 1.576000 \\
\hline & -2.322610 & 1.023520 & 1.302059 \\
\hline & -3.110584 & 1.503963 & 2.353557 \\
\hline & -2.677976 & 1.318283 & 3.673624 \\
\hline & -1.461721 & 0.717738 & 3. \\
\hline & 2.631674 & -1.201069 & 248 \\
\hline & 1.124733 & -0.623581 & 4.984480 \\
\hline & 3.308471 & -1.656623 & 4.488918 \\
\hline & -2.631674 & 1.201069 & 0.277248 \\
\hline & -3.308471 & 1.656623 & 918 \\
\hline & -1.124733 & 0.623581 & 480 \\
\hline & 3.110584 & 1.503963 & -2.353557 \\
\hline & 2.322610 & 1.023520 & -1.302059 \\
\hline & 1.117584 & 0.380361 & -1.576000 \\
\hline & 0.677068 & 0.271409 & -2.907139 \\
\hline & 1.461721 & 0.717738 & -3 . \\
\hline & 2.677976 & 1.318283 & -3.673624 \\
\hline & -0.677068 & -0.271409 & -2.907139 \\
\hline & -1.117584 & -0.380361 & -1.576000 \\
\hline & -2.322610 & -1.023520 & -1.30205 \\
\hline & -3.110584 & -1.503963 & -2.353557 \\
\hline & -2.677976 & -1.318283 & -3.673624 \\
\hline & -1.461721 & -0.717738 & -3.957117 \\
\hline & 2.631674 & 1.201069 & -0.277248 \\
\hline & 1.124733 & 0.623581 & -4.984480 \\
\hline & 3.308471 & 1.656623 & -4.48891 \\
\hline & -2.631674 & -1.201069 & -0.27724 \\
\hline & -3.308471 & -1.656623 & -4 \\
\hline & -1.124733 & 3581 & -4.98448 \\
\hline & -15.752281 & $1-1.248284$ & -0.6661 \\
\hline & -15.752281 & 1.24828 & 0.666106 \\
\hline & 15.752281 & -1.248284 & 0.66610 \\
\hline & 15.752281 & 1.248284 & -0.666106 \\
\hline & -15.945668 & $3-0.054279$ & -1.371489 \\
\hline & -15.945668 & $\begin{array}{ll}3 & 0.054279\end{array}$ & 1.371489 \\
\hline & 15.945668 & -0.054279 & 1.37148 \\
\hline & 15.945668 & 0.054279 & -1.371489 \\
\hline & -15.923702 & $2-1.167896$ & 0.720776 \\
\hline & -15 & 1.167896 & -0.720776 \\
\hline & 23702 & -1.167896 & -0.720776 \\
\hline & 15.923702 & 1.167896 & 0.72077 \\
\hline & -15.964138 & $3-0.051290$ & -2.455 \\
\hline
\end{tabular}

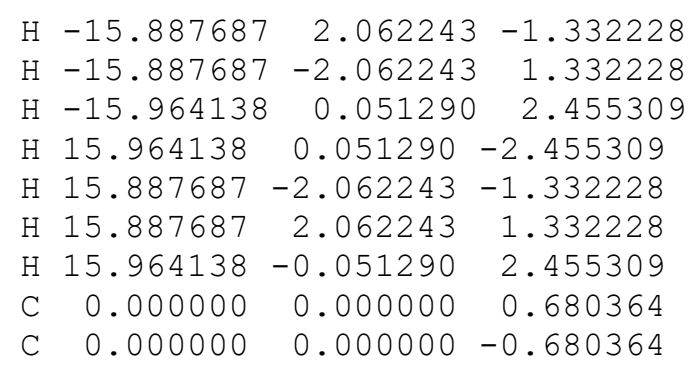

$\Delta \mathrm{H}=-4230.523191$ 


\begin{tabular}{|c|c|c|c|}
\hline C & 13.166501 & -3.898973 & 2.625142 \\
\hline C & 14.486563 & -3.532512 & 2.425728 \\
\hline $\mathrm{C}$ & 15.190078 & -3.952012 & 1.293584 \\
\hline C & 4.572061 & -4.894920 & 0.465004 \\
\hline $\mathrm{C}$ & 3.245881 & -5.255029 & 0.659487 \\
\hline & 8.447771 & -3.950672 & -0.386551 \\
\hline & 10.843295 & -4.456527 & -0.412391 \\
\hline & 0.793898 & -4.934597 & 3.845482 \\
\hline & 8.417121 & -4.349329 & 3.879893 \\
\hline & 6.145698 & -4.884287 & 3.292435 \\
\hline & 3.974628 & -3.776457 & 3.544875 \\
\hline & 5.092278 & -0.893532 & 0.585151 \\
\hline & 7.269491 & -1.988762 & 0.339269 \\
\hline & 12.615879 & -3.448756 & 3.444523 \\
\hline & 14.927415 & -2.796442 & 3.089201 \\
\hline & 15.110561 & -5.299412 & -0.386546 \\
\hline & 12.771671 & -5.936932 & -0.039818 \\
\hline & 8.257276 & 4.068891 & -1.752773 \\
\hline & 8.956730 & 4.163339 & -0.5 \\
\hline & 10.307770 & 4.474111 & -0.531407 \\
\hline & 11.010199 & 4.707948 & -1.71 \\
\hline & 10.285125 & 4.721654 & -2.910438 \\
\hline & 8.935482 & 4.404622 & -2.927843 \\
\hline C & 12.493834 & 4.695488 & -1.697029 \\
\hline & 6.884305 & 3.510926 & -1.810615 \\
\hline $\mathrm{C}$ & 5.928329 & 3.998073 & -2.704427 \\
\hline & 4.701511 & 3.369945 & -2.848950 \\
\hline $\mathrm{C}$ & 4.379803 & 2.230209 & -2.1 \\
\hline $\mathrm{C}$ & 5.311803 & 1.779332 & -1.17 \\
\hline $\mathrm{C}$ & 6.54 & 2.404203 & -1.02 \\
\hline C & 13.166501 & 3.898973 & -2.625142 \\
\hline C & 14.48 & 3.532512 & -2.42 \\
\hline C & 15.190078 & 3.952012 & -1.293584 \\
\hline C & 14.57 & 4.894920 & -0.4 \\
\hline $\mathrm{C}$ & 13.245881 & 5.255029 & -0.659487 \\
\hline $\mathrm{H}$ & 8.447771 & 3.950672 & 0.386551 \\
\hline $\mathrm{H}$ & 10.843295 & 4.456527 & 0.412391 \\
\hline $\mathrm{H}$ & 10.793898 & 4.934597 & -3.84 \\
\hline $\mathrm{H}$ & 8.417121 & 4.349329 & -3.879893 \\
\hline $\mathrm{H}$ & 6.145698 & 4.884287 & -3.292435 \\
\hline $\mathrm{H}$ & 3.974628 & 3.776457 & -3.544875 \\
\hline $\mathrm{H}$ & 5.092278 & 0.893532 & -0.585151 \\
\hline $\mathrm{H}$ & 7.269491 & 1.988762 & -0.339269 \\
\hline $\mathrm{H}$ & 12.615879 & 3.448756 & -3.444523 \\
\hline $\mathrm{H}$ & 14.927415 & 2.796442 & -3.089201 \\
\hline $\mathrm{H}$ & 15.110561 & 5.299412 & 0.386546 \\
\hline $\mathrm{H}$ & 12.771671 & 5.936932 & 0.039818 \\
\hline $\mathrm{C}$ & -8.257276 & -4.068891 & -1.752773 \\
\hline$C$ & -8.956730 & -4.163339 & -0.548305 \\
\hline $\mathrm{C}$ & -10.307770 & -4.474111 & -0.531407 \\
\hline C & -11.010199 & $9-4.707948$ & -1.716817 \\
\hline $\mathrm{C}$ & -10.285125 & $5-4.721654$ & -2.910438 \\
\hline $\mathrm{C}$ & -8.935482 & -4.404622 & -2.927843 \\
\hline $\mathrm{C}$ & -12.493834 & $4-4.695488$ & -1.697029 \\
\hline $\mathrm{C}$ & -6.884305 & -3.510926 & -1.810615 \\
\hline $\mathrm{C}$ & -5.928329 & -3.998073 & -2.704427 \\
\hline & -4.701511 & -3.369945 & -2.848950 \\
\hline
\end{tabular}

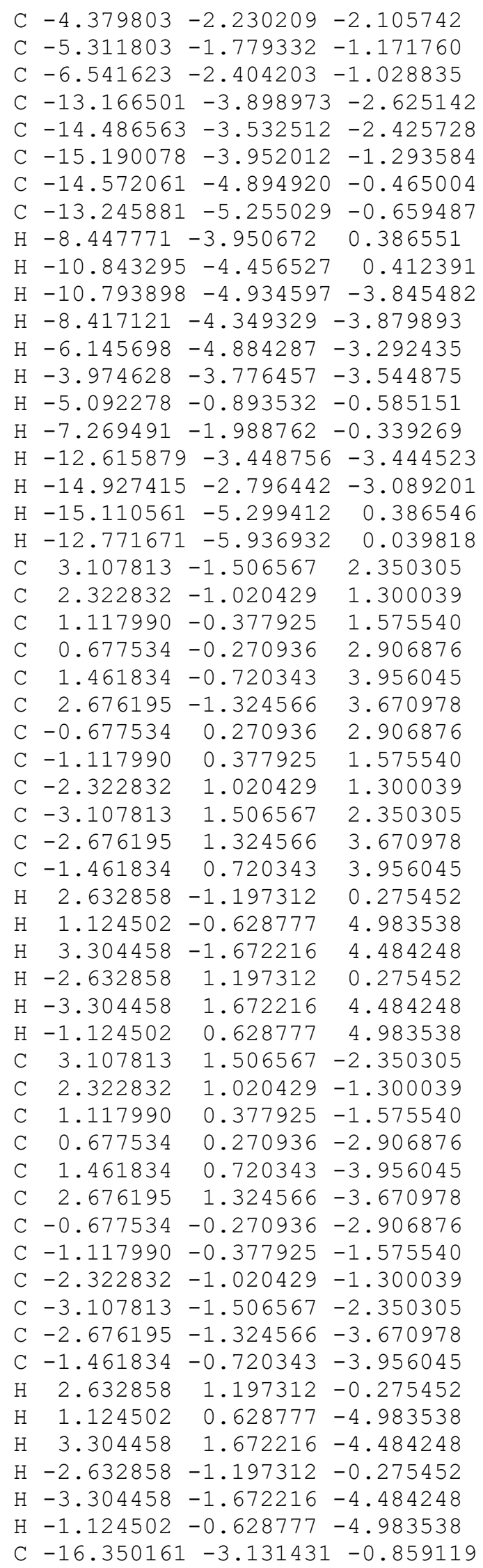




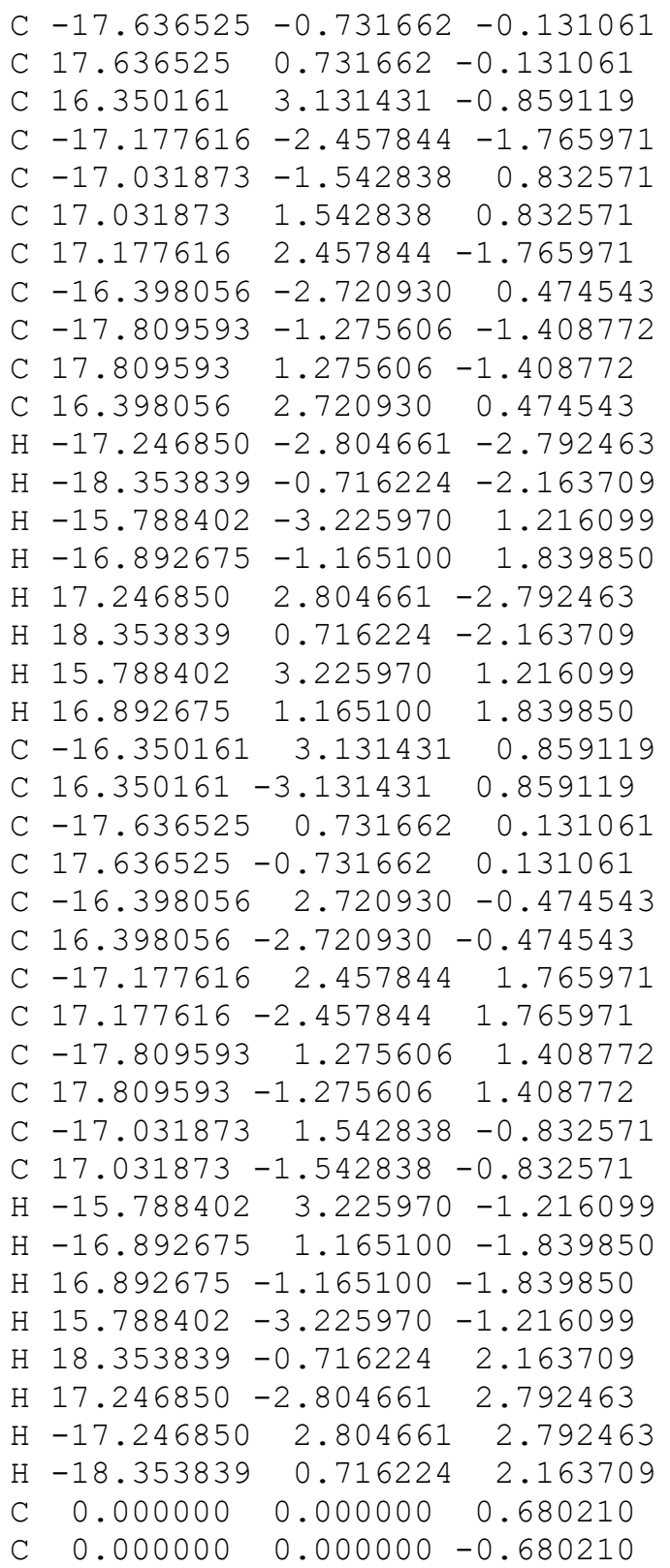

\section{$\Delta \mathrm{H}=-4692.411619$}

\section{3-4}

$\begin{array}{lrrr}\text { C } & 0.084620 & 9.129393 & -1.599180 \\ \text { C } & 0.000000 & 9.762616 & -0.356008 \\ \text { C } & 1.095990 & 10.436251 & 0.155258 \\ \text { C } & 2.300863 & 10.487320 & -0.562189 \\ \text { C } & 2.195299 & 10.255821 & -1.941731 \\ \text { C } & 1.107681 & 9.558996 & -2.450437 \\ \text { C } & -0.471010 & 7.762274 & -1.776685 \\ \text { C } & -0.936683 & 7.203782 & -2.966937 \\ \text { C } & -1.046491 & 5.825092 & -3.110277 \\ \text { C } & -0.672249 & 4.942508 & -2.087183 \\ \text { C }-0.312921 & 5.515696 & -0.865188\end{array}$

\begin{tabular}{|c|c|c|c|}
\hline C & -0.220030 & 6.885609 & -0.713444 \\
\hline $\mathrm{H}$ & -0.843697 & 9.559999 & 0.296336 \\
\hline $\mathrm{H}$ & 1.068327 & 10.715609 & 1.201892 \\
\hline & 3.050176 & 10.406559 & -2.591824 \\
\hline $\mathrm{H}$ & 1.165269 & 9.172020 & -3.463427 \\
\hline & -1.182401 & 7.847123 & -3.806432 \\
\hline & -1.406363 & 5.439390 & -4.057754 \\
\hline & -0.020108 & 4.897613 & -0.025159 \\
\hline $\mathrm{H}$ & 0.166512 & 7.278137 & 0.22 \\
\hline & -0.084620 & -9.129393 & -1.599180 \\
\hline & 0.000000 & -9.762616 & -0.356008 \\
\hline & -1.095990 & -10.43 & 0.15 \\
\hline & -2.300863 & -10.48 & -0 \\
\hline & -2.195299 & -10.2 & -1.9 \\
\hline C & -1.107681 & -9.55 & -2.45 \\
\hline C & 0.471010 & -7.762274 & -1.776685 \\
\hline & 0.93 & -7.203782 & -2.96 \\
\hline C & 1.046491 & -5.825092 & -3.11 \\
\hline C & 0.672 & -4.94 & -2.0 \\
\hline C & 0.312 & -5.51 & -0.86 \\
\hline $\mathrm{C}$ & 0.22 & -6.88 & -0.7 \\
\hline & 0.843 & -9.55 & 0.29 \\
\hline & $-1 \cdot 0$ & -10.71 & 1.2 \\
\hline & -3 . & -10.4 & -2 \\
\hline & -1.16 & -9.17 & -3.4 \\
\hline & 1.1 & -7.84 & -3 \\
\hline $\mathrm{H}$ & 1.40 & -5.43 & -4.05 \\
\hline & 0 . & -4 & -0 . \\
\hline $\mathrm{H}$ & -0.166512 & $-7.27 \xi$ & 0.22 \\
\hline C & -4 & -7 . & \\
\hline C & -4.979602 & -8.14 & 0.04 \\
\hline $\mathrm{C}$ & -4 & -9.29 & -0.5 \\
\hline $\mathrm{C}$ & -3.551365 & -10.09 & 0.1 \\
\hline$C$ & -3 & -9 & \\
\hline $\mathrm{C}$ & -4.019769 & -8.762928 & 2.13 \\
\hline $\mathrm{C}$ & -4.28 & -6.33 & 1.62 \\
\hline $\mathrm{C}$ & -4.482315 & -5.678679 & 2.83 \\
\hline $\mathrm{C}$ & -3.85 & -4.46 & 3.0 \\
\hline $\mathrm{C}$ & -2.98 & -3.87 & 2.16 \\
\hline $\mathrm{C}$ & -2.86 & -4.49 & 0.9 \\
\hline $\mathrm{C}$ & -3.50 & -5.695740 & 0.66 \\
\hline $\mathrm{H}$ & -5.52 & -7.44 & -0.5 \\
\hline 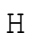 & -4.63 & -9.44 & -1.58 \\
\hline $\mathrm{H}$ & -2.93 & -10.5 & 739 \\
\hline 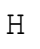 & -3.77 & $-8.54 \varepsilon$ & 3.17 \\
\hline $\mathrm{H}$ & -5.11 & -6.12 & 3.6 \\
\hline $\mathrm{H}$ & -4.03 & -3.98 & 4.04 \\
\hline $\mathrm{H}$ & -2.20 & -4.09 & 0.1 \\
\hline $\mathrm{H}$ & -3.30 & -6.19 & -0.28 \\
\hline $\mathrm{C}$ & 4.58 & 7.76 & 1.3 \\
\hline $\mathrm{C}$ & 4.9796 & 8.144 & 0.04 \\
\hline $\mathrm{C}$ & 4.47 & 9.2 & -0.52 \\
\hline $\mathrm{C}$ & 3.551 & 10.09 & 0.17 \\
\hline $\mathrm{C}$ & 3.52 & 9.92 & 1.563076 \\
\hline $\mathrm{C}$ & 4.0 & 8.7 & 2.1 \\
\hline $\mathrm{C}$ & 4.2 & 6.3 & 1.6 \\
\hline $\mathrm{C}$ & 4.48 & 5.67 & 2.8 \\
\hline
\end{tabular}




\begin{tabular}{|c|c|c|c|}
\hline & 3.855058 & 4.463839 & 3.095378 \\
\hline C & 2.984915 & 3.873037 & 2.169581 \\
\hline & 2.863389 & 4.499738 & 0.927259 \\
\hline & 3.501579 & 5.695740 & 0.661564 \\
\hline & 5.525587 & 7.443361 & -0.574531 \\
\hline & 4.631478 & 9.441087 & -1.58595 \\
\hline & 2.938149 & 10.591022 & 2.186739 \\
\hline & 3.777821 & 8.548077 & 3.172395 \\
\hline & 5.110261 & 6.126464 & 3.603433 \\
\hline & 4.038384 & 3.982538 & 4.049809 \\
\hline & 2.205042 & 4.095979 & 0.166370 \\
\hline & 3.304336 & 6.194051 & -0.281379 \\
\hline & 0.537640 & -3.474107 & -2.297974 \\
\hline & 0.383240 & -2.585534 & -1.217192 \\
\hline & 0.126282 & -1.237655 & -1.420039 \\
\hline & 0.096695 & -0.733327 & -2.736122 \\
\hline & 0.263597 & -1.580894 & -3.814871 \\
\hline & 0.458457 & -2.940416 & -3.587251 \\
\hline & -0.096695 & 0.733327 & -2.736122 \\
\hline & -0.126282 & 1.237655 & -1.420039 \\
\hline & -0.383240 & 2.585534 & -1.217192 \\
\hline & -0.537640 & 3.474107 & -2.297974 \\
\hline & -0.458457 & 2.940416 & -3.587251 \\
\hline & -0.263597 & 1.580894 & -3.814871 \\
\hline & 0.465416 & -2.951165 & -0.199452 \\
\hline & 0.235877 & -1.207599 & -4.833899 \\
\hline & 0.531871 & -3.595882 & -4.447506 \\
\hline & -0.465416 & 2.951165 & -0.199452 \\
\hline & -0.531871 & 3.595882 & -4.447506 \\
\hline & -0.235877 & 1.207599 & -4.833899 \\
\hline & -2.136887 & -2.695709 & 2.501684 \\
\hline 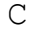 & -1.560784 & -1.922225 & 1.489525 \\
\hline & -0.676690 & -0.905644 & 1.830516 \\
\hline C & -0.432325 & -0.578039 & 3.173003 \\
\hline & -1.018065 & -1.333926 & 4.182892 \\
\hline & -1.839748 & -2.392092 & 3.843135 \\
\hline C & 0.432325 & 0.578039 & 3.173003 \\
\hline C & 0.676690 & 0.905644 & 1.830516 \\
\hline C & 1.560784 & 1.922225 & 1.489525 \\
\hline C & 2.136887 & 2.695709 & 2.501684 \\
\hline C & 1.839748 & 2.392092 & 3.843135 \\
\hline C & 1.018065 & 1.333926 & 4.182892 \\
\hline $\mathrm{N}$ & 0.000000 & 0.000000 & 0.987958 \\
\hline $\mathrm{H}$ & -1.810351 & -2.095528 & 0.450469 \\
\hline $\mathrm{H}$ & -0.824271 & -1.105748 & 5.225898 \\
\hline & -2.247008 & -3.008636 & 4.635458 \\
\hline & 1.810351 & 2.095528 & 0.450469 \\
\hline & 2.247008 & 3.008636 & 4.635458 \\
\hline & 0.824271 & 1.105748 & 0.243898 \\
\hline & & 00000 & 45 \\
\hline
\end{tabular}

$\Delta \mathrm{H}=-2848.31895$

\section{3-5}

$\begin{array}{llll}C & -0.395081 & 9.210813 & -1.870860\end{array}$ C $-0.75287210 .015703-0.786979$
C $\quad 0.000000 \quad 11.132272-0.455174$

C $1.17204211 .443790-1.159553$

C $\quad 1.35685910 .791452-2.383700$

C $\quad 0.580519 \quad 9.699189-2.739153$

C $-0.729688 \quad 7.764027-1.941861$

C $-1.250661 \quad 7.127380-3.066195$

C $-1.248181 \quad 5.741914-3.160964$

C $-0.692228 \quad 4.938194 \quad-2.157834$

C $-0.250236 \quad 5.581520-0.998635$

C $-0.278456 \quad 6.962003-0.889294$

$\mathrm{H}-1.567122 \quad 9.709464-0.137408$

$\mathrm{H}-0.258092 \quad 11.679421 \quad 0.445222$

H $2.225858 \quad 11.013057-2.992188$

$\mathrm{H} \quad 0.852783 \quad 9.120344 \quad-3.616292$

$\mathrm{H}-1.642106 \quad 7.720753-3.886762$

$\mathrm{H}-1.674847 \quad 5.285197-4.047228$

$\mathrm{H} \quad 0.180653 \quad 5.010328-0.183726$

$\mathrm{H} \quad 0.139822 \quad 7.433740-0.005862$

C $0.395081-9.210813-1.870860$

C $0.752872-10.015703-0.786979$

C $0.000000-11.132272-0.455174$

C $-1.172042-11.443790-1.159553$

C $-1.356859-10.791452-2.383700$

C $-0.580519-9.699189-2.739153$

C $0.729688-7.764027-1.941861$

C $\quad 1.250661-7.127380-3.066195$

C $\quad 1.248181-5.741914-3.160964$

C $\quad 0.692228 \quad-4.938194-2.157834$

C $0.250236-5.581520-0.998635$

C $0.278456-6.962003-0.889294$

$\mathrm{H} \quad 1.567122-9.709464-0.137408$

H $\quad 0.258092-11.679421 \quad 0.445222$

$\mathrm{H}-2.225858-11.013057-2.992188$

$\mathrm{H}-0.852783-9.120344-3.616292$

H $\quad 1.642106-7.720753-3.886762$

$\mathrm{H} \quad 1.674847 \quad-5.285197 \quad-4.047228$

$\mathrm{H}-0.180653-5.010328-0.183726$

$\mathrm{H}-0.139822-7.433740-0.005862$

C $-5.578031-7.182691 \quad 1.505414$

$\begin{array}{llll}\text { C }-5.818433 & -7.703830 & 0.232917\end{array}$

$\begin{array}{llll}\text { C } & -5.814808 & -9.071648 & 0.020203\end{array}$

C $-5.583336-9.965300 \quad 1.073547$

C $-5.647788-9.444753 \quad 2.371347$

$\begin{array}{llll}\text { C }-5.629427 & -8.072777 & 2.582774\end{array}$

C $-4.790618-11.182691 \quad 0.742780$

C $-4.966672-5.839770 \quad 1.663674$

C $-5.212538-4.980132 \quad 2.734936$

C $-4.410830-3.867025 \quad 2.952196$

C $-3.325661-3.575409 \quad 2.117815$

C $-3.131556-4.394739 \quad 1.004575$

$\begin{array}{llll}\text { C }-3.933030 & -5.500561 & 0.782926\end{array}$

C $-3.645723-11.397034 \quad 1.515609$

C $-2.458398-11.788672 \quad 0.922535$

C $-2.378746-11.978298-0.460382$

C $-3.601934-12.159312-1.125188$

C $-4.792023-11.762262-0.533859$

H $-5.873361-7.035308-0.620408$ 


\begin{tabular}{|c|c|c|c|}
\hline $\mathrm{H}$ & -5.816243 & -9.433610 & -1.001971 \\
\hline $\mathrm{H}$ & -5.558347 & -10.107748 & 3.226291 \\
\hline 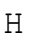 & -5.507278 & -7.697929 & 3.594522 \\
\hline $\mathrm{H}$ & -6.030889 & -5.190216 & 3.417060 \\
\hline $\mathrm{H}$ & -4.631004 & -3.217468 & 3.793081 \\
\hline $\mathrm{H}$ & -2.301752 & -4.208073 & 0.330735 \\
\hline $\mathrm{H}$ & -3.696604 & -6.161997 & -0.043769 \\
\hline $\mathrm{H}$ & -3.603452 & -11.031986 & 2.535491 \\
\hline $\mathrm{H}$ & -1.557492 & -11.715642 & \\
\hline $\mathrm{H}$ & -3.619157 & -12.460232 & -2.167745 \\
\hline $\mathrm{H}$ & -5.697215 & -11.755825 & $5-1.133442$ \\
\hline $\mathrm{C}$ & 5.578031 & 7.182691 & 1.505414 \\
\hline$C$ & 5.818433 & 7.703830 & 0.232917 \\
\hline $\mathrm{C}$ & 5.814808 & 9.071648 & 0.020203 \\
\hline r & 5.583336 & 9.965300 & 1.073547 \\
\hline$C$ & 5.647788 & 9.444753 & 2.371347 \\
\hline$C$ & 5.629427 & 8.072777 & 2.582774 \\
\hline $\mathrm{C}$ & 4.790618 & 11.182691 & 0.742780 \\
\hline C & 4.966672 & 5.839770 & 1.663674 \\
\hline $\mathrm{C}$ & 5.212538 & 4.980132 & 2.734936 \\
\hline $\mathrm{C}$ & 4.41 & 3.867025 & 2.952 \\
\hline $\mathrm{C}$ & 3.325661 & 3.575409 & 2.117815 \\
\hline $\mathrm{C}$ & 3.13 & 4.394739 & 1.004 \\
\hline $\mathrm{C}$ & 3.933030 & 5.500561 & 0.782926 \\
\hline $\mathrm{C}$ & 3.64 & 11.397034 & 1.51 \\
\hline $\mathrm{C}$ & 2.45 & 11.788672 & 0.922535 \\
\hline $\mathrm{C}$ & 2.37 & 11.978298 & -0.46 \\
\hline $\mathrm{C}$ & 3.60 & 12.159312 & -1.12 \\
\hline C & 4.79 & 11.762262 & -0.533 \\
\hline $\mathrm{H}$ & 5.873361 & 7.03 & -0.62 \\
\hline $\mathrm{H}$ & 5.81 & 9.433610 & -1.001971 \\
\hline $\mathrm{H}$ & 5.55 & 10.107 & 3.22 \\
\hline $\mathrm{H}$ & 5.507278 & 7.697929 & 3.59 \\
\hline $\mathrm{H}$ & 6.03 & 5.15 & 3.4 \\
\hline $\mathrm{H}$ & 4.631004 & 3.217468 & 3.793 \\
\hline $\mathrm{H}$ & 2.30 & 4.208073 & 0.33 \\
\hline $\mathrm{H}$ & 3.696604 & 6.161997 & -0.043769 \\
\hline $\mathrm{H}$ & 3.603452 & 11.031986 & 2.535 \\
\hline $\mathrm{H}$ & 1.557492 & 11.715642 & 1.519653 \\
\hline $\mathrm{H}$ & 3.619157 & 12.460232 & -2.167 \\
\hline $\mathrm{H}$ & 5.697215 & 11.755825 & -1.133442 \\
\hline $\mathrm{C}$ & 0.499367 & -3.475205 & -2.348268 \\
\hline C & 0.333789 & -2.593789 & -1.265094 \\
\hline $\mathrm{C}$ & 0.092910 & -1.242013 & -1.471438 \\
\hline C & 0.078953 & -0.735731 & -2.786680 \\
\hline $\mathrm{C}$ & 0.228921 & -1.583544 & -3.867332 \\
\hline C & 0.414974 & -2.943182 & -3.638459 \\
\hline $\mathrm{C}$ & -0.078953 & 0.735731 & -2.786680 \\
\hline C & -0.092910 & 1.242013 & -1.471438 \\
\hline $\mathrm{C}$ & -0.333789 & 2.593789 & -1.265094 \\
\hline C & -0.499367 & 3.475205 & -2.34826 \\
\hline C & -0.414974 & 2.943182 & -3.638459 \\
\hline C & -0.228921 & 1.583544 & -3.867332 \\
\hline $\mathrm{H}$ & 0.407994 & -2.967088 & -0.248201 \\
\hline $\mathrm{H}$ & 0.201962 & -1.209627 & -4.886120 \\
\hline $\mathrm{H}$ & 0.482770 & -3.603776 & -4.495444 \\
\hline $\mathrm{H}$ & -0.407994 & 2.967088 & -0.24820 \\
\hline
\end{tabular}

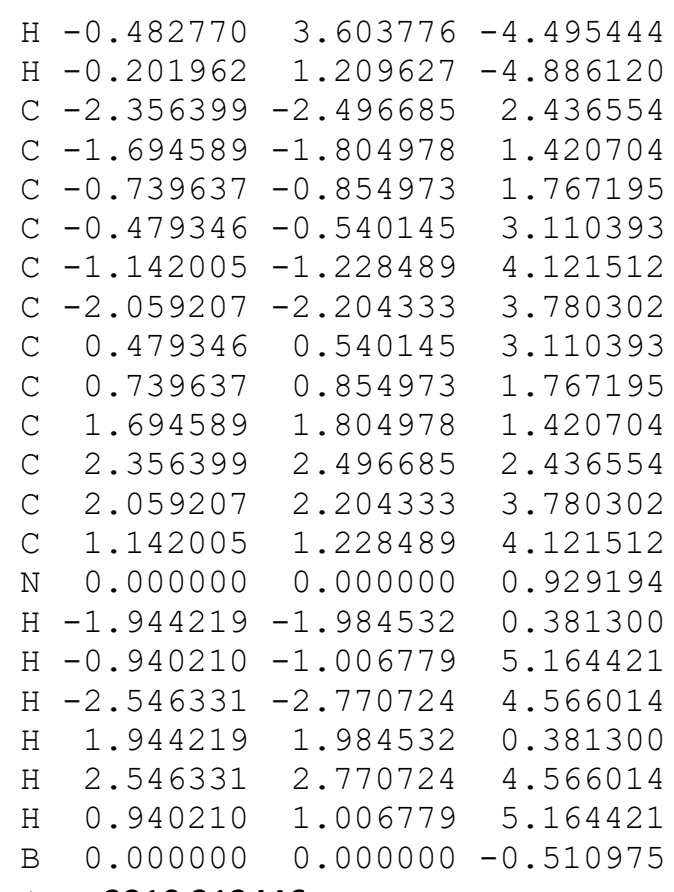

$\Delta \mathrm{H}=-3310.213446$ 


$\begin{array}{rrrr}\text { C } & 0.479953 & 0.539153 & 3.266680 \\ \text { C } & 0.739424 & 0.856007 & 1.923930 \\ \text { C } & 1.697022 & 1.805333 & 1.579015 \\ \text { C } & 2.367962 & 2.482366 & 2.598338 \\ \text { C } & 2.070508 & 2.188487 & 3.941703 \\ \text { C } & 1.145241 & 1.220321 & 4.280557 \\ \text { N } & 0.000000 & 0.000000 & 1.086488 \\ \text { H } & -1.946543 & -1.990876 & 0.540267 \\ \text { H } & -0.942185 & -0.995838 & 5.322612 \\ \text { H } & -2.567495 & -2.746637 & 4.727224 \\ \text { H } & 1.946543 & 1.990876 & 0.540267 \\ \text { H } & 2.567495 & 2.746637 & 4.727224 \\ \text { H } & 0.942185 & 0.995838 & 5.322612 \\ \text { B } & 0.000000 & 0.000000 & -0.353210 \\ \text { A } & -3772108756 & & \end{array}$

$\Delta \mathrm{H}=-3772.108756$

\section{3-7}

\begin{tabular}{|c|c|c|c|}
\hline & -3 & 8.381884 & -1.653911 \\
\hline & -3 . & 9.143888 & -0.488202 \\
\hline & -3.998896 & 10.518143 & -0.521510 \\
\hline & -4.273630 & 11.195243 & -1.715320 \\
\hline & -4.519772 & 10.413074 & -2.847973 \\
\hline & -4.343722 & 9.036063 & -2.820015 \\
\hline & -4.006460 & 12.656008 & -1 \\
\hline & -3.455256 & 6.979378 & -1.674900 \\
\hline & -4.028038 & 91374 & -2.478358 \\
\hline & -3.457695 & 4.729861 & -2.573180 \\
\hline & -2.296311 & 4.406081 & -1.865914 \\
\hline & -1.753705 & 5.377342 & -1.025496 \\
\hline & -2.317209 & 6.639074 & -0.936447 \\
\hline & -3.427857 & 13.218925 & -2.920203 \\
\hline & -2.665686 & 14.373708 & -2.843190 \\
\hline & -2.410498 & 14.982807 & -1.609302 \\
\hline & -3.208703 & 14.573763 & -0.535032 \\
\hline & -4.006883 & 13.445127 & -0.623269 \\
\hline & -3.591270 & 8.663779 & 0 . \\
\hline & -3.803011 & 11.077877 & 0.385580 \\
\hline & -4.786340 & 10.887043 & -3.787235 \\
\hline & -4.445882 & 8.474095 & -3.743231 \\
\hline & -4.934535 & 6.209002 & -3.034681 \\
\hline & -3.927151 & 3.978970 & -3.2011 \\
\hline & 2769 & 5.161328 & -0.477237 \\
\hline & -1.828461 & 7.391317 & -0.326483 \\
\hline & -3.451621 & 12.681514 & -3.862234 \\
\hline & -2.154545 & 14.716947 & -3.736456 \\
\hline & -3.099197 & 15.047976 & 0.43408 \\
\hline & -4.534849 & 13.111566 & 0.263815 \\
\hline & 3.936712 & -8.381884 & -1.653911 \\
\hline & 3.846257 & -9.143888 & -0.488202 \\
\hline & 3.998896 & -10.518143 & $3-0.521510$ \\
\hline & 4.273630 & -11.195243 & $3-1.71532$ \\
\hline & 4.519772 & -10.413074 & -2.847973 \\
\hline & 4.343722 & -9.036063 & -2.8200 \\
\hline & 4.006460 & $-12 \cdot 656$ & -1.77 \\
\hline & & & \\
\hline
\end{tabular}

$\begin{array}{llll}\text { C } \quad 4.028038 & -5.991374 & -2.478358\end{array}$

C $\quad 3.457695-4.729861-2.573180$

C $\quad 2.296311-4.406081-1.865914$

C $\quad 1.753705-5.377342-1.025496$

C $\quad 2.317209-6.639074-0.936447$

C $3.427857-13.218925-2.920203$

C $2.665686-14.373708-2.843190$

C $2.410498-14.982807-1.609302$

C $\quad 3.208703-14.573763-0.535032$

C $4.006883-13.445127-0.623269$

$\mathrm{H} \quad 3.591270 \quad-8.663779 \quad 0.450940$

H $\quad 3.803011-11.077877 \quad 0.385580$

$\mathrm{H} \quad 4.786340-10.887043-3.787235$

$\mathrm{H} \quad 4.445882-8.474095 \quad-3.743231$

$\mathrm{H} \quad 4.934535-6.209002-3.034681$

$\mathrm{H} \quad 3.927151-3.978970-3.201134$

$\mathrm{H} \quad 0.842769-5.161328-0.477237$

$\mathrm{H} \quad 1.828461-7.391317-0.326483$

$\mathrm{H} \quad 3.451621-12.681514 \quad-3.862234$

$\mathrm{H} \quad 2.154545-14.716947 \quad-3.736456$

$\mathrm{H} \quad 3.099197-15.047976 \quad 0.434080$

$\mathrm{H} \quad 4.534849-13.111566 \quad 0.263815$

$\begin{array}{llll}\text { C } & -3.794284 & -8.319695 & 1.906658\end{array}$

$\begin{array}{llll}\text { C }-3.946635 & -8.914096 & 0.653068\end{array}$

$\begin{array}{llll}\text { C } & -4.101072 & -10.285706 & 0.530571\end{array}$

C $-4.112878-11.115460 \quad 1.655912$

$\begin{array}{llll}\text { C } & -4.094754 & -10.505564 & 2.912338\end{array}$

$\begin{array}{llll}\text { C } & -3.935929 & -9.132635 & 3.034733\end{array}$

$\begin{array}{llll}\text { C }-3.851737 & -12.564614 & 1.466024\end{array}$

$\begin{array}{llll}C & -3.342332 & -6.913314 & 2.040001\end{array}$

$\begin{array}{llll}C & -3.830055 & -6.062744 & 3.033635\end{array}$

$\begin{array}{llll}C & -3.277052 & -4.806597 & 3.231256\end{array}$

$\begin{array}{llll}\text { C }-2.210715 & -4.352869 & 2.448560\end{array}$

$\begin{array}{llll}\text { C }-1.762679 & -5.181844 & 1.419906\end{array}$

$\begin{array}{llll}\text { C }-2.316007 & -6.436792 & 1.219160\end{array}$

$\begin{array}{llll}\text { C } & -2.831749 & -13.153164 & 2.215395\end{array}$

$\begin{array}{llll}\text { C } & -2.188279 & -14.294624 & 1.766577\end{array}$

$\begin{array}{llll}\text { C } & -2.544741 & -14.892853 & 0.554969\end{array}$

C $-3.718684-14.441671-0.060471$

$\begin{array}{llll}\text { C } & -4.356156 & -13.291914 & 0.382559\end{array}$

$\mathrm{H}-3.888622-8.302142-0.241606$

$\mathrm{H}-4.116369-10.729087-0.459741$

$\mathrm{H}-4.140678-11.117779 \quad 3.807774$

$\mathrm{H}-3.838788 \quad-8.696034 \quad 4.023913$

$\mathrm{H}-4.655541-6.387325 \quad 3.659678$

$\mathrm{H}-3.684515 \quad-4.163589 \quad 4.004644$

$\mathrm{H}-0.930072-4.860970 \quad 0.802282$

$\mathrm{H}-1.901906-7.082550 \quad 0.451254$

$\mathrm{H}-2.424479-12.628151 \quad 3.073168$

$\mathrm{H}-1.290024-14.618286 \quad 2.281120$

$\mathrm{H}-4.081289-14.934138-0.957566$

$\mathrm{H}-5.207179-12.908675-0.172301$

C $\quad 3.794284 \quad 8.319695 \quad 1.906658$

$\begin{array}{llll}\text { C } & 3.946635 & 8.914096 & 0.653068\end{array}$

C $\quad 4.101072 \quad 10.285706 \quad 0.530571$

$\begin{array}{llll}\text { C } \quad 4.112878 & 11.115460 & 1.655912\end{array}$

C $\quad 4.094754 \quad 10.505564 \quad 2.912338$ 


\begin{tabular}{|c|c|c|c|}
\hline & 3.935929 & 9.132635 & 4733 \\
\hline & 3.851737 & 12.564614 & 466024 \\
\hline & 3.342332 & 6.913314 & 2 $\cap \wedge \cap \cap \cap 1$ \\
\hline & 3.830055 & 6.062744 & \\
\hline & $3.27^{\circ}$ & 4.806597 & \\
\hline & 2.210715 & 4.352869 & \\
\hline & 1.762679 & 5.181844 & \\
\hline & 2.316007 & 6.436 & \\
\hline & 2.831749 & 13.1 & \\
\hline & 2.188279 & 14.294624 & \\
\hline & 2.544741 & 14.892 & \\
\hline & 3.718684 & 14.441671 & \\
\hline & 4.35 & 13.25 & \\
\hline & 3.888622 & 8.302 & -0 . \\
\hline & 4.11 & 10.72 & \\
\hline & 4.14 & 11.11 & \\
\hline & 3.8 & 8.6 & \\
\hline & 4.6555 & 6.38 & 3 . \\
\hline & 3.6 & 4.1 & \\
\hline & 0.93 & 4.8 & 0 . \\
\hline & 1.9 & 7. & \\
\hline & 2.42 & 12.62 & \\
\hline & 1.2 & 14.6 & 2 . \\
\hline & 4.08 & 14 . & -0 . \\
\hline & 5.2 & 12 . & \\
\hline & 1.61 & -3.1 & -2 \\
\hline & 1.10 & $-2 \cdot 3$ & -0 \\
\hline & 0.45 & $-1 \cdot 1$ & -1 \\
\hline & 0.32 & -0.6 & -2 \\
\hline & 0.81 & $-1 \cdot 3$ & \\
\hline & 1.45 & $-2 \cdot 5$ & -3 \\
\hline & -0.32 & 0.6 & -2 . \\
\hline & -0.45 & 1.1 & -1 \\
\hline & $-1 \cdot 1$ & & \\
\hline & $-1 \cdot 6$ & 3.1 & -2 \\
\hline & -1 & & \\
\hline & -0.81 & 1.3 & -3 \\
\hline & 1.2 & -2.7 & \\
\hline & 0.71 & -0 & -4 \\
\hline & 1.8 & -3 & -4 \\
\hline & -1.25 & 2.7 & 0 . \\
\hline & -1.8 & 3.1 & $-4 \cdot 1$ \\
\hline & -0.71 & 0.9 & -4 \\
\hline & $-1 \cdot 5$ & -3 & \\
\hline & $-1 \cdot 0$ & $-2 \cdot 2$ & 1.7 \\
\hline & -0.4 & $-1 \cdot 0$ & \\
\hline & -0.30 & -0.6 & 3. \\
\hline & -0.7 & -1.4 & 4. \\
\hline & -1.34 & -2.6 & 4. \\
\hline & 0.3 & 0. & 3. \\
\hline & 0.454 & 1.0 & 2.0 \\
\hline & 1.090 & 2.2 & 1.7 \\
\hline & 1.5392 & 3.0 & 2.7 \\
\hline & 1.3428 & 2.6 & 4.0 \\
\hline & $0.73^{7}$ & & \\
\hline & 0.000 & 0 . & 1. \\
\hline & -1.274 & -2 & \\
\hline
\end{tabular}

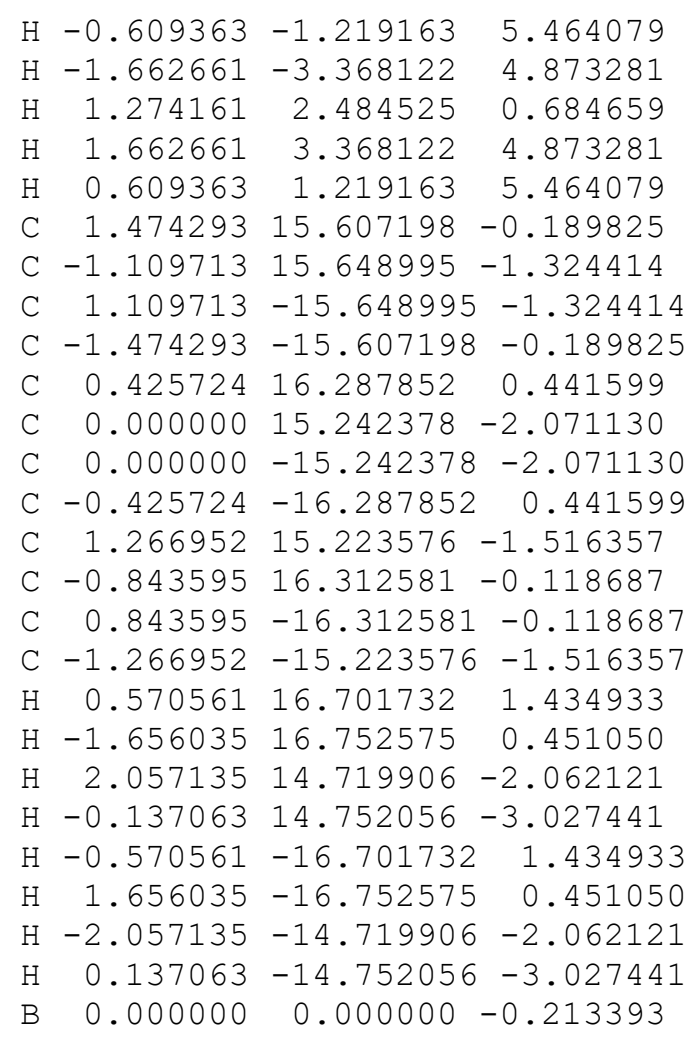

$\Delta \mathrm{H}=-4233.990678$

\section{3-8}

C $-4.563975 \quad 8.064561-1.719085$

C $-4.679616 \quad 8.818901-0.550183$

C $-5.038044 \quad 10.157190-0.598642$

C $-5.299351 \quad 10.791299-1.816402$

C $-5.29425710 .007217-2.972143$

C $-4.930282 \quad 8.670043-2.924233$

C $-5.328493 \quad 12.273949-1.869306$

C $-3.948697 \quad 6.715070-1.711617$

C $-4.403453 \quad 5.691115-2.544947$

C $-3.722137 \quad 4.487056-2.631159$

C $-2.560010 \quad 4.258638-1.888465$

C $-2.138200 \quad 5.260256-1.013922$

C $-2.817388 \quad 6.466295-0.929312$

C $-4.548825 \quad 12.921137-2.829259$

C $-4.21004614 .256252-2.689960$

C $-4.64196815 .000379-1.588870$

C $-5.572758 \quad 14.401183-0.733081$

C $-5.904610 \quad 13.060262-0.866815$

$\mathrm{H}-4.444064 \quad 8.365135 \quad 0.407340$

$\mathrm{H}-5.034899 \quad 10.739615 \quad 0.317101$

H $-5.527851 \quad 10.461248-3.930171$

$\mathrm{H}-4.860627 \quad 8.106754-3.849490$

$\mathrm{H}-5.305171 \quad 5.837005-3.131494$

$\mathrm{H}-4.103174 \quad 3.705363-3.280752$

$\mathrm{H}-1.233746 \quad 5.116558-0.431392$

$\mathrm{H}-2.426940 \quad 7.249934-0.288071$ 


\begin{tabular}{|c|c|c|c|}
\hline $\mathrm{H}$ & -4.087309 & 12.343735 & -3.623539 \\
\hline $\mathrm{H}$ & -3.483635 & 14.681739 & -3.373768 \\
\hline & -5.988038 & 14.968745 & 0.094034 \\
\hline & -6.575256 & 12.603594 & -0.145292 \\
\hline & 4.563975 & -8.064561 & -1.719085 \\
\hline & 4.679616 & -8.818901 & -0.550183 \\
\hline & 5.038044 & -10.157190 & -0.598642 \\
\hline & 5.299351 & -10.791299 & -1.816402 \\
\hline & 5.294257 & -10.007217 & -2.972143 \\
\hline & 4.930282 & -8.670043 & -2.924233 \\
\hline & 5.328493 & -12.273949 & -1.869306 \\
\hline & 3.948697 & -6.715070 & -1.711617 \\
\hline & 4.403453 & -5.691115 & -2.544947 \\
\hline & 3.722137 & -4.487056 & -2.631159 \\
\hline & 2.560010 & -4.258638 & -1.888465 \\
\hline & 2.138200 & -5.260256 & -1.013922 \\
\hline & 2.817388 & -6.466295 & -0.929 \\
\hline & 4.548825 & -12.921137 & -2.82 \\
\hline & 4.21 & -14.256252 & -2.6 \\
\hline & 4.641968 & -15.000379 & -1.5 \\
\hline & 5.57 & -14.40 & -0.7 \\
\hline & 5.904610 & -13.06 & -0.8 \\
\hline & 4.44 & -8.365 & 0.40 \\
\hline & 5.03 & -10.73 & \\
\hline & 5.52 & -10.4 & -3.9 \\
\hline & 4.86 & -8.10 & -3.8 \\
\hline & 5.30 & -5.83 & -3.13 \\
\hline & 4.10 & -3.70 & -3.28 \\
\hline & 1.23 & -5.116558 & -0.43 \\
\hline & 2.42 & -7.24 & -0.2 \\
\hline & 4.08 & $-12 \cdot 34$ & -3.6 \\
\hline & 3. & $-14 \cdot 6$ & -3 \\
\hline & 5.98 & -14.96 & 34 \\
\hline & 6.57 & $-12 \cdot 6$ & -0 \\
\hline$C$ & -3.691990 & -8.366553 & 1.84 \\
\hline 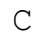 & -3.75 & -9.004 & 0.6 \\
\hline C & -3.996464 & $-10 \cdot 366170$ & 0.51 \\
\hline 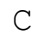 & -4.19 & -11.14 & 1. \\
\hline C & -4.237399 & -10.484707 & 2.8 \\
\hline C & -3.98 & -9.123880 & 2.97 \\
\hline C & -4.116605 & -12.620675 & 1.557907 \\
\hline $\mathrm{C}$ & -3.21 & -6.969646 & 1.97 \\
\hline C & -3.74 & -6.092411 & 2.918180 \\
\hline C & -3.19 & -4.839860 & 3.12 \\
\hline $\mathrm{C}$ & -2.080055 & -4.412885 & 2.398366 \\
\hline $\mathrm{C}$ & -1.57 & -5.267468 & 1.41 \\
\hline $\mathrm{C}$ & -2.128664 & -6.522932 & 1.211434 \\
\hline . & -3.29 & -13.309162 & 2.4 \\
\hline $\mathrm{C}$ & -2.87 & -14.603386 & 2.181975 \\
\hline C & -3.277679 & $-15 \cdot 2$ & 1.01 \\
\hline C & -4.243106 & -14.639312 & 0.221396 \\
\hline $\mathrm{C}$ & -4.65 & -13.338633 & 0.483343 \\
\hline 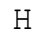 & -3.56 & -8.435242 & -0.303346 \\
\hline $\mathrm{H}$ & -3.955787 & -10.848241 & -0.458948 \\
\hline $\mathrm{H}$ & -4.422 & -11.053217 & 3.795450 \\
\hline & -3.956 & -8.655700 & 3.958560 \\
\hline & -4.619 & -6.392288 & 3.494907 \\
\hline
\end{tabular}

\begin{tabular}{|c|c|c|c|}
\hline $\mathrm{H}$ & -3.638425 & -4.175688 & 3.860898 \\
\hline $\mathrm{H}$ & -0.706337 & -4.965038 & 0.843155 \\
\hline H & -1.673837 & -7.189095 & 0.485236 \\
\hline & -2.858931 & -12.784555 & 3.294074 \\
\hline $\mathrm{H}$ & -2.124751 & -15.050817 & 2.821308 \\
\hline $\mathrm{H}$ & -4.630915 & -15.148765 & -0.655422 \\
\hline H & -5.351774 & -12.854905 & -0.189646 \\
\hline C & 3.691990 & 8.366553 & 1.843079 \\
\hline C & 3.753501 & 9.004163 & 0.602677 \\
\hline C & 3.996464 & 10.366170 & 0.512575 \\
\hline C & 4.193008 & 11.142295 & 1.65 \\
\hline C & 4.237399 & 10.484707 & 2.88 \\
\hline C & 3.988131 & 9.123880 & 2.9 \\
\hline C & 4.116605 & 12.620675 & 1.55 \\
\hline C & 3.211240 & 6.969646 & 1.97 \\
\hline C & 3.749682 & 6.092411 & 2.918180 \\
\hline C & 3.194302 & 4.839860 & 3.12 \\
\hline C & 2.080055 & 4.412885 & 2.398366 \\
\hline C & 1.576 & 5.267468 & 1.41 \\
\hline $\mathrm{C}$ & 2.128664 & 6.522932 & 1.21 \\
\hline C & 3.291171 & 13.309162 & 2.4 \\
\hline $\mathrm{C}$ & 2.878054 & 14.603386 & 2.18 \\
\hline $\mathrm{C}$ & 3.277679 & 15.264686 & 1.0 \\
\hline $\mathrm{C}$ & 4.24 & 539312 & 0.22 \\
\hline $\mathrm{C}$ & 4.650358 & 13.338633 & 0.483343 \\
\hline $\mathrm{H}$ & 3.5699 & 8.435242 & -0.3 \\
\hline $\mathrm{H}$ & 3.955787 & 10.848241 & -0.458948 \\
\hline $\mathrm{H}$ & 4.422424 & 11.053217 & 3.7 \\
\hline $\mathrm{H}$ & 3.956210 & 8.655700 & 3.958560 \\
\hline $\mathrm{H}$ & 4.6190 & 6.392288 & 3.4 \\
\hline $\mathrm{H}$ & 3.638425 & 4.175688 & 3.860898 \\
\hline $\mathrm{H}$ & 0.7 & 4.965038 & 0.8 \\
\hline $\mathrm{H}$ & 1.673837 & 7.189095 & 0.485236 \\
\hline $\mathrm{H}$ & 2 . & 12.784555 & 3.2 \\
\hline $\mathrm{H}$ & 2.124751 & 15.050817 & 2.821308 \\
\hline $\mathrm{H}$ & 4.630915 & 15.148765 & -0.65 \\
\hline $\mathrm{H}$ & 5.351774 & 12.854905 & -0.189646 \\
\hline $\mathrm{C}$ & 1.776312 & -3.013155 & -2.07 \\
\hline $\mathrm{C}$ & 1.228564 & -2.307255 & -0.992425 \\
\hline $\mathrm{C}$ & 0.512134 & -1.135760 & -1.20 \\
\hline C & 0.350952 & -0.652114 & -2.517910 \\
\hline $\mathrm{C}$ & 0.864530 & -1.345822 & -3.59 \\
\hline $\mathrm{C}$ & 1.569087 & -2.523178 & -3.365694 \\
\hline $\mathrm{C}$ & -0.350952 & 0.652114 & -2.51 \\
\hline $\mathrm{C}$ & -0.512134 & 1.135760 & -1.203389 \\
\hline $\mathrm{C}$ & $-1 \cdot 2$ & 2.307255 & -0.9 \\
\hline $\mathrm{C}$ & -1.776312 & 3.013155 & -2.074316 \\
\hline C & -1.56 & 2.523178 & -3.3 \\
\hline $\mathrm{C}$ & -0.864530 & 1.345822 & -3.597535 \\
\hline $\mathrm{H}$ & 1.39 & -2.667098 & 0.019077 \\
\hline $\mathrm{H}$ & 0.742696 & -0.980629 & -4.612541 \\
\hline $\mathrm{H}$ & 1.962671 & -3.080533 & -4.209845 \\
\hline $\mathrm{H}$ & -1.398559 & 2.667098 & 0.019077 \\
\hline $\mathrm{H}$ & -1.962671 & 3.080533 & -4.209845 \\
\hline $\mathrm{H}$ & -0.742696 & 0.980629 & -4.612541 \\
\hline $\mathrm{C}$ & -1.431712 & -3.113322 & 2.701460 \\
\hline $\mathrm{C}$ & -1.000385 & -2.266639 & 1.68 \\
\hline
\end{tabular}




\begin{tabular}{|c|c|c|c|}
\hline $\mathrm{C}$ & -0.411248 & -1.053691 & 2.028242 \\
\hline 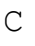 & -0.275977 & -0.667209 & 3.370881 \\
\hline$C$ & -0.691598 & -1.523457 & 4.385407 \\
\hline $\mathrm{C}$ & -1.258969 & -2.736291 & 4.045654 \\
\hline$C$ & 0.275977 & 0.667209 & 3.370881 \\
\hline 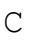 & 0.411248 & 1.053691 & 2.028242 \\
\hline $\mathrm{C}$ & 1.000385 & 2.266639 & 1.681418 \\
\hline 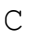 & 1.431712 & 3.113322 & 2.701460 \\
\hline $\mathrm{C}$ & 1.258969 & 2.736291 & 5654 \\
\hline C & 0.691598 & 1.523457 & 4.385407 \\
\hline $\mathrm{N}$ & 0.000000 & 0.000000 & 1.191908 \\
\hline $\mathrm{H}$ & -1.167376 & -2.532279 & 0.6 \\
\hline $\mathrm{H}$ & -0.580804 & -1.240918 & 5.4 \\
\hline $\mathrm{H}$ & -1.570649 & -3.419967 & 4.827749 \\
\hline $\mathrm{H}$ & 1.167376 & 2.532279 & 0.643258 \\
\hline $\mathrm{H}$ & 1.570649 & 3.419967 & 4.827749 \\
\hline $\mathrm{H}$ & 0.580804 & 1.240918 & 5.42 \\
\hline C & 2.425021 & 16.378917 & 0.52 \\
\hline C & 0.00 & 17.578862 & -0.2 \\
\hline $\mathrm{C}$ & 0.000000 & -17.578862 & -0.2 \\
\hline $\mathrm{C}$ & -2.425021 & -16.378917 & 0 . \\
\hline $\mathrm{C}$ & 1.730956 & 17.231530 & 1.39 \\
\hline C & 0.82 & 16.94 & $-1 \cdot 1$ \\
\hline $\mathrm{C}$ & -0.82 & -16.945607 & -1 \\
\hline $\mathrm{C}$ & -1.73 & -17.23 & 076 \\
\hline $\mathrm{C}$ & 2.015482 & 16.354295 & -0.8 \\
\hline $\mathrm{C}$ & 0.53 & 17.82 & 1.0 \\
\hline $\mathrm{C}$ & -0.53 & -17.820964 & 334 \\
\hline $\mathrm{C}$ & -2.01 & -16.354295 & -0 . \\
\hline $\mathrm{H}$ & 2.074247 & 17.355869 & 2.41 \\
\hline $\mathrm{H}$ & -0.03 & 18.38 & 1.7 \\
\hline $\mathrm{H}$ & 2.53 & 15.721 & -1.51 \\
\hline $\mathrm{H}$ & 0.452336 & 16.752085 & -2.1 \\
\hline $\mathrm{H}$ & -2.074247 & -17.355869 & \\
\hline $\mathrm{H}$ & 0.03 & $-18 \cdot 38$ & 258 \\
\hline $\mathrm{H}$ & -2.535553 & -15.72 & -1 \\
\hline $\mathrm{H}$ & -0.452336 & -16.752085 & -2.1 \\
\hline C & -3.8 & 16.19 & $-1 \cdot 2$ \\
\hline C & 3.84 & -16.19 & -1.20 \\
\hline C & -1.462102 & 17.54 & -0.5 \\
\hline C & 1.462102 & -17.542977 & -0.52 \\
\hline C & -3.434809 & 16.303126 & 0.1 \\
\hline C & 3.434809 & -16.303126 & 0.127238 \\
\hline C & -3.176800 & 16.988374 & -2.14 \\
\hline $\mathrm{C}$ & 3.176800 & -16.98 & -2.14 \\
\hline C & -2.004664 & 17.65 & -1.81 \\
\hline C & 2.004664 & -17.652095 & -1.812482 \\
\hline C & -2.267100 & 16.969064 & 0.460 \\
\hline C & 2.267100 & -16.969064 & 0.460058 \\
\hline $\mathrm{H}$ & -3.932801 & 15.718960 & 0.8 \\
\hline $\mathrm{H}$ & -1.890438 & 16.879667 & 1.473384 \\
\hline $\mathrm{H}$ & 1.890438 & -16.879667 & 1.473384 \\
\hline $\mathrm{H}$ & 3.932801 & -15.718960 & 0.893619 \\
\hline $\mathrm{H}$ & 1.450991 & -18.171174 & -2.589046 \\
\hline $\mathrm{H}$ & 3.521656 & -17.008220 & -3.173755 \\
\hline $\mathrm{H}$ & -3.521656 & 17.008220 & -3.1 \\
\hline & -1.450991 & 18.171174 & -2.5 \\
\hline
\end{tabular}

\section{B $0.000000 \quad 0.000000-0.246106$ $\Delta \mathrm{H}=-4695.879797$}

\begin{tabular}{|c|c|c|c|}
\hline $\mathrm{C}$ & -2.414502 & 2.414746 & -2.113456 \\
\hline $\mathrm{C}$ & -1.747518 & 1.747837 & -1.097225 \\
\hline $\mathrm{C}$ & -0.803528 & 0.803879 & -1.477134 \\
\hline $\mathrm{C}$ & -0.513472 & 0.513715 & -2.821744 \\
\hline $\mathrm{C}$ & -1.199154 & 1.199185 & -3.822998 \\
\hline $\mathrm{C}$ & -2.145401 & 2.145480 & -3.461120 \\
\hline $\mathrm{C}$ & 0.513472 & -0.513715 & -2.821744 \\
\hline $\mathrm{C}$ & 0.803528 & -0.803879 & -1.477134 \\
\hline $\mathrm{C}$ & 1.747518 & -1.747837 & -1.097225 \\
\hline $\mathrm{C}$ & 2.414502 & -2.414746 & -2.113456 \\
\hline $\mathrm{C}$ & 2.145401 & -2.145480 & -3.461120 \\
\hline $\mathrm{C}$ & 1.199154 & -1.199185 & -3.822998 \\
\hline N & 0.000000 & 0.000000 & -0.677153 \\
\hline $\mathrm{H}$ & -1.948472 & 1.948637 & -0.050797 \\
\hline $\mathrm{H}$ & -0.996123 & 0.996055 & -4.869491 \\
\hline $\mathrm{H}$ & -2.686073 & 2.686240 & -4.230212 \\
\hline $\mathrm{H}$ & 1.948472 & -1.948637 & -0.050797 \\
\hline $\mathrm{H}$ & 2.686073 & -2.686240 & -4.230212 \\
\hline $\mathrm{H}$ & 0.996123 & -0.996055 & -4.869491 \\
\hline $\mathrm{C}$ & 2.414502 & 2.414746 & 2.113456 \\
\hline C & 1.747518 & 1.747837 & 1.097225 \\
\hline $\mathrm{C}$ & 0.803528 & 0.803879 & 1.477134 \\
\hline C & 0.513472 & 0.513715 & 2.821744 \\
\hline $\mathrm{C}$ & 1.199154 & 1.199185 & 3.822998 \\
\hline $\mathrm{C}$ & 2.145401 & 2.145480 & 3.461120 \\
\hline $\mathrm{C}$ & -0.513472 & -0.513715 & 2.821744 \\
\hline $\mathrm{C}$ & -0.803528 & -0.803879 & 1.477134 \\
\hline $\mathrm{C}$ & -1.747518 & -1.747837 & 1.097225 \\
\hline $\mathrm{C}$ & -2.414502 & -2.414746 & 2.113456 \\
\hline $\mathrm{C}$ & -2.145401 & -2.145480 & 3.461120 \\
\hline $\mathrm{C}$ & -1.199154 & -1.199185 & 3.822998 \\
\hline N & 0.000000 & 0.000000 & 0.677153 \\
\hline $\mathrm{H}$ & 1.948472 & 1.948637 & 0.050797 \\
\hline $\mathrm{H}$ & 0.996123 & 0.996055 & 4.869491 \\
\hline $\mathrm{H}$ & 2.686073 & 2.686240 & 4.230212 \\
\hline $\mathrm{H}$ & -1.948472 & -1.948637 & 0.050797 \\
\hline $\mathrm{H}$ & -2.686073 & -2.686240 & 4.230212 \\
\hline $\mathrm{H}$ & -0.996123 & -0.996055 & 4.869491 \\
\hline $\mathrm{H}$ & 3.159826 & -3.160113 & -1.857609 \\
\hline $\mathrm{H}$ & -3.159826 & 3.160113 & -1.857609 \\
\hline $\mathrm{H}$ & -3.159826 & -3.160113 & 1.857609 \\
\hline $\mathrm{H}$ & 3.159826 & 3.160113 & 1.85760 \\
\hline
\end{tabular}
$\Delta \mathrm{H}=-1033.203408$
$4 b$
C -1.078759
2.790442
3.691446
C -1.247117
3.204380
C -0.893127
2. 375431
2. 372006
C -0.330074
1.129917
1. 313481
C -0.226210
0.696067
1.580393
2.914968 


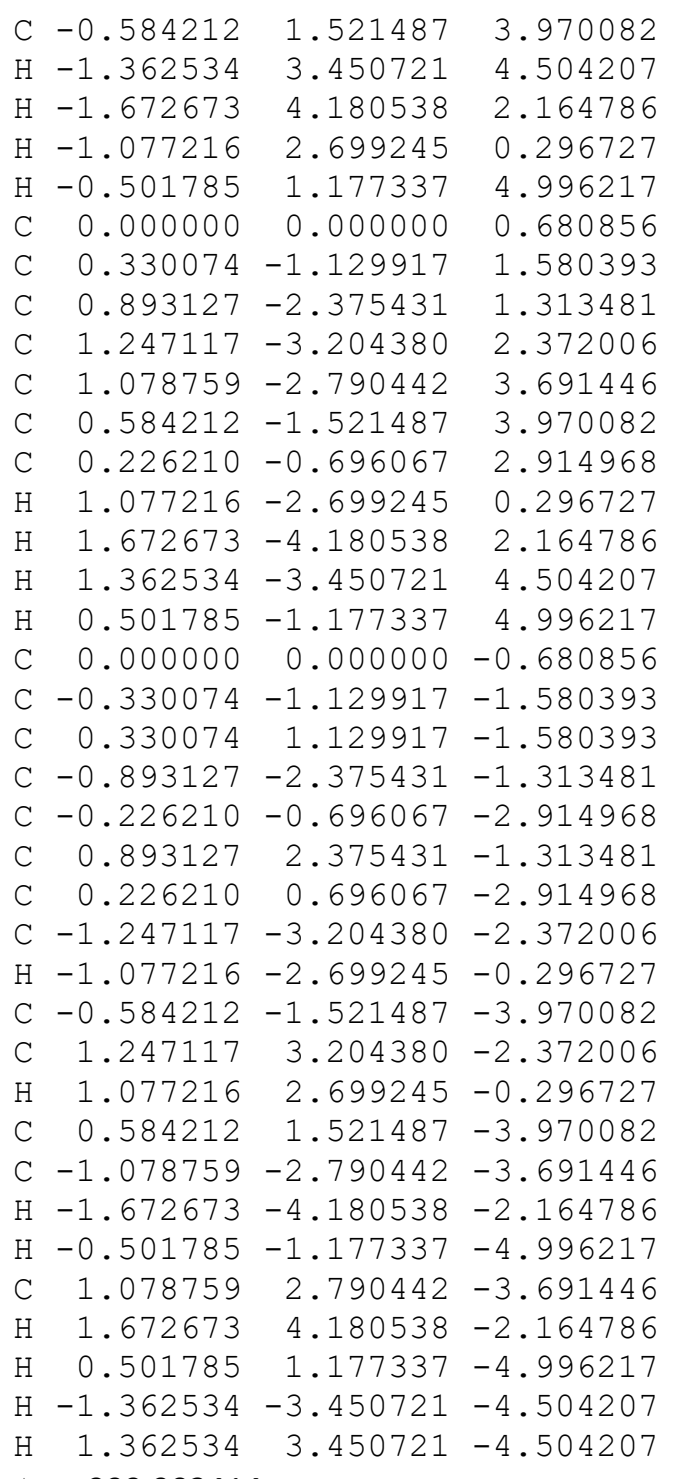

$\Delta \mathrm{H}=-999.903414$

\section{4c}

$\begin{array}{rrrr}\text { C } & -2.479728 & 2.443938 & -2.479030 \\ \mathrm{C} & -1.826020 & 1.872733 & -1.387506 \\ \mathrm{C} & -0.820820 & 0.933834 & -1.589157 \\ \mathrm{C} & -0.512524 & 0.537367 & -2.907599 \\ \mathrm{C} & -1.157664 & 1.100336 & -3.994751 \\ \mathrm{C} & -2.139271 & 2.066370 & -3.771471 \\ \mathrm{C} & 0.512524 & -0.537367 & -2.907599 \\ \mathrm{C} & 0.820820 & -0.933834 & -1.589157 \\ \mathrm{C} & 1.826020 & -1.872733 & -1.387506 \\ \mathrm{C} & 2.479728 & -2.443938 & -2.479030 \\ \mathrm{C} & 2.139271 & -2.066370 & -3.771471 \\ \mathrm{C} & 1.157664 & -1.100336 & -3.994751 \\ \mathrm{H} & -2.111406 & 2.165419 & -0.382123 \\ \mathrm{H} & -0.922698 & 0.792041 & -5.008906 \\ \mathrm{H} & -2.650361 & 2.516155 & -4.616734\end{array}$

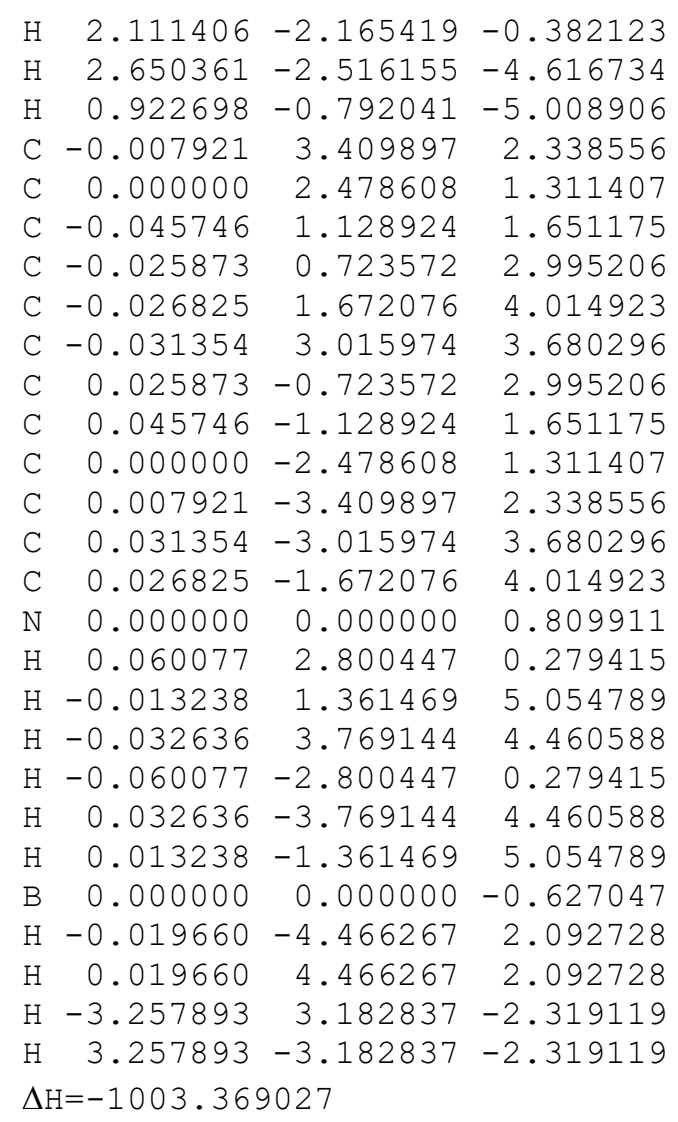

\section{$5 \mathrm{NN}-4$}

$\begin{array}{rrrr}\text { C } & 7.791357 & 2.739579 & -0.597366 \\ \text { C } & 8.242078 & 2.493248 & 0.708738 \\ \text { C } & 9.442638 & 0.730058 & -0.111212 \\ \text { C } & 9.366117 & 1.289622 & -1.398267 \\ \text { C } & 6.400334 & 3.135690 & -0.891645 \\ \text { C } & 5.953728 & 3.357033 & -2.199509 \\ \text { C } & 3.855249 & 2.721349 & -1.575841 \\ \text { C } & 4.247266 & 2.785426 & -0.234360 \\ \text { H } & 7.882480 & 3.050389 & 1.565400 \\ \text { H } & 9.906524 & 0.884466 & -2.245481 \\ \text { H } & 6.636928 & 3.652630 & -2.987461 \\ \text { H } & 3.537675 & 2.613591 & 0.566234 \\ \text { C } & -7.791357 & -2.739579 & -0.597366 \\ \text { C } & -8.242078 & -2.493248 & 0.708738 \\ \text { C } & -9.442638 & -0.730058 & -0.111212 \\ \text { C } & -9.366117 & -1.289622 & -1.398267 \\ \text { C } & -6.400334 & -3.135690 & -0.891645 \\ \text { C } & -5.953728 & -3.357033 & -2.199509 \\ \text { C } & -3.855249 & -2.721349 & -1.575841 \\ \text { C } & -4.247266 & -2.785426 & -0.234360 \\ \text { H } & -7.882480 & -3.050389 & 1.565400 \\ \text { H } & -9.906524 & -0.884466 & -2.245481 \\ \text { H } & -6.636928 & -3.652630 & -2.987461 \\ \text { H } & -3.537675 & -2.613591 & 0.566234 \\ \text { C } & -7.791357 & 2.739579 & 0.597366\end{array}$




\begin{tabular}{|c|c|c|c|}
\hline C & -8.242078 & 2.493248 & -0.708738 \\
\hline & -9.442638 & 0.730058 & \\
\hline & -9.366117 & 1.289622 & \\
\hline & 6.400334 & 3.135690 & \\
\hline & 5.95 & 3.357033 & \\
\hline & 3.855249 & 2.721349 & \\
\hline & 4.247266 & 2.785426 & \\
\hline & 7 . & & \\
\hline & 4 & 0.88 & \\
\hline & 6.636928 & 3.6526 & \\
\hline & 3.537675 & 2.61 & \\
\hline & 91357 & -2.739579 & \\
\hline & 78 & -2.4 & \\
\hline & 638 & -0.73 & \\
\hline & 117 & -1.28 & \\
\hline & 34 & -3 & \\
\hline & 28 & -3 & \\
\hline & 249 & -2.72 & \\
\hline & 4 & -2.7 & \\
\hline & 7 & -3 & -1 \\
\hline & & -0 . & \\
\hline & 6 . & -3 & \\
\hline & & -2 . & \\
\hline & -2 . & -2 & -2 \\
\hline C & -0 & -0.7 & -1 \\
\hline & -0 . & -0.4 & -2 \\
\hline C & -1 & $-1 \cdot 1$ & -3 \\
\hline & -2 . & -1 . & \\
\hline z & & 0 . & -2 \\
\hline & 0 & & -1 \\
\hline C & 2 . & 2 . & -2 \\
\hline$z$ & 2 & & \\
\hline E & 1. & 1. & -3 \\
\hline N & & & \\
\hline H & -1 & -0 . & -4 \\
\hline & & -2 . & \\
\hline & 2.97 & 2 . & -4 \\
\hline H & 1. & 0 . & -4 \\
\hline $\mathrm{C}$ & -2 . & 2 . & \\
\hline $\mathrm{C}$ & -0 . & 0 . & \\
\hline C & -0 . & 0.46 & \\
\hline $\mathrm{C}$ & -1 & 1.1 & \\
\hline C & -2 . & 1. & \\
\hline $\mathrm{C}$ & 0 . & -0.4 & \\
\hline & 0 . & -0.72 & \\
\hline C & 2 . & -2.0 & \\
\hline & 2 . & $-1 \cdot 9$ & \\
\hline $\mathrm{C}$ & 1 . & $-1 \cdot 1$ & \\
\hline & 0 . & 0. & \\
\hline $\mathrm{H}$ & -1 & 0 . & \\
\hline & -2 . & 2.43 & \\
\hline $\mathrm{H}$ & 2 . & -2.43 & \\
\hline & 1. & -0.98 & \\
\hline $\mathrm{N}$ & 9450 & -1.4 & 1. \\
\hline & -1 & -1 & -1 \\
\hline$v$ & & 1. & -1 \\
\hline & -1 & 1. & \\
\hline
\end{tabular}

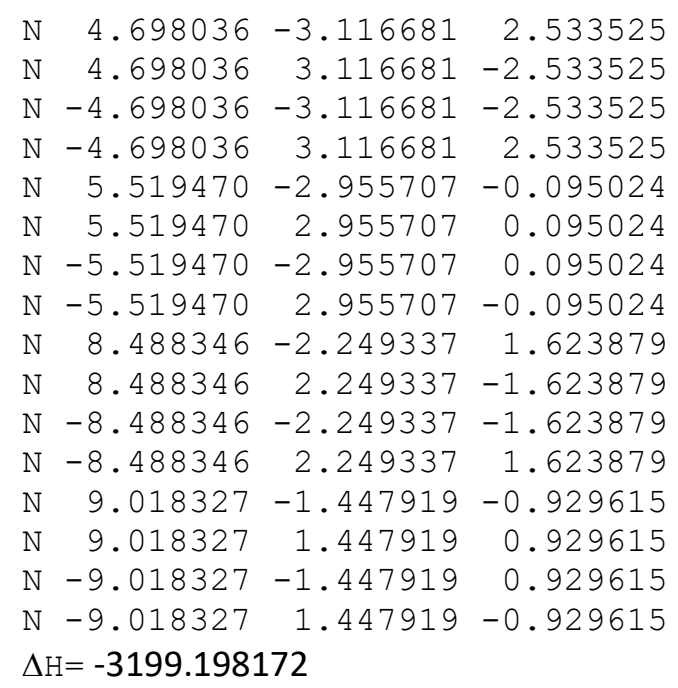

5NN- 5

$\begin{array}{lrrr}\mathrm{C} & -7.641151 & 3.672682 & 0.575359 \\ \mathrm{C} & -8.143469 & 3.463262 & -0.715232 \\ \mathrm{C} & -10.013819 & 2.535025 & 0.200813 \\ \mathrm{C} & -9.666493 & 3.084962 & 1.443146 \\ \mathrm{C} & -6.192419 & 3.683466 & 0.863149 \\ \mathrm{C} & -5.707416 & 3.854498 & 2.163500 \\ \mathrm{C} & -3.736492 & 2.890088 & 1.555348 \\ \mathrm{C} & -4.146013 & 2.925019 & 0.218114 \\ \mathrm{H} & -7.560991 & 3.695598 & -1.598698 \\ \mathrm{H} & -10.319234 & 3.009770 & 2.304826 \\ \mathrm{H} & -6.332343 & 4.277299 & 2.941429 \\ \mathrm{H} & -3.487245 & 2.588780 & -0.573762 \\ \mathrm{C} & 7.641151 & -3.672682 & 0.575359 \\ \mathrm{C} & 8.143469 & -3.463262 & -0.715232 \\ \mathrm{C} & 10.013819 & -2.535025 & 0.200813 \\ \mathrm{C} & 9.666493 & -3.084962 & 1.443146 \\ \mathrm{C} & 6.192419 & -3.683466 & 0.863149 \\ \mathrm{C} & 5.707416 & -3.854498 & 2.163500 \\ \mathrm{C} & 3.736492 & -2.890088 & 1.555348 \\ \mathrm{C} & 4.146013 & -2.925019 & 0.218114 \\ \mathrm{H} & 7.560991 & -3.695598 & -1.598698 \\ \mathrm{H} & 10.319234 & -3.009770 & 2.304826 \\ \mathrm{H} & 6.332343 & -4.277299 & 2.941429 \\ \mathrm{H} & 3.487245 & -2.588780 & -0.573762 \\ \mathrm{C} & 7.641151 & 3.672682 & -0.575359 \\ \mathrm{C} & 8.143469 & 3.463262 & 0.715232 \\ \mathrm{C} & 10.013819 & 2.535025 & -0.200813 \\ \mathrm{C} & 9.666493 & 3.084962 & -1.443146 \\ \mathrm{C} & 10.846359 & 1.321212 & -0.088933 \\ \mathrm{C} & 6.192419 & 3.683466 & -0.863149 \\ \mathrm{C} & 5.707416 & 3.854498 & -2.163500 \\ \mathrm{C} & 3.736492 & 2.890088 & -1.555348 \\ \mathrm{C} & 4.146013 & 2.925019 & -0.218114 \\ \mathrm{C} & 11.079492 & -0.690904 & -1.143930 \\ \mathrm{C} & 10.846359 & -1.321212 & 0.088933 \\ \mathrm{C} & 11.079492 & 0.690904 & 1.143930\end{array}$




\begin{tabular}{|c|c|c|c|}
\hline & .560991 & 95598 & 98 \\
\hline & 10.319234 & 3.009770 & -2.304826 \\
\hline & 6.332343 & 4.277299 & -2.941429 \\
\hline & 3.487245 & 2.588780 & 0.573762 \\
\hline & 11.191316 & -1.244978 & -2.068077 \\
\hline & 11.191316 & 1.244978 & 2.068077 \\
\hline & -7.641151 & -3.672682 & -0.575359 \\
\hline & -8.12 & -3.463262 & 0.715232 \\
\hline & & & $-c$ \\
\hline & & & -1 \\
\hline & & & \\
\hline & -6.192419 & -3.683466 & -0.863149 \\
\hline & -5.707416 & -3 & 3500 \\
\hline & -3.73 & -2.890088 & -1.5 \\
\hline & -4.1 & -2.925019 & -0.218114 \\
\hline & -11 & 0904 & -1 \\
\hline & -10.846359 & 1.321212 & 0.08893 \\
\hline & -11.079492 & -0.690904 & 1.143930 \\
\hline & -7.5 & -3.695598 & 1.598698 \\
\hline & -10 & -3 & -2.304826 \\
\hline & -6.332343 & -4.277299 & -2.94142 \\
\hline & -3.487245 & -2.588780 & 0.573762 \\
\hline & -11.191316 & 1.244978 & -2.068077 \\
\hline & -11.191316 & -1.244978 & 2.068077 \\
\hline & 2.534 & -2.138396 & 1.9 \\
\hline & 0.85 & -0.7 & 098 \\
\hline & 0.536874 & -0.480072 & 2.827764 \\
\hline 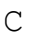 & 1.270052 & -1.152398 & 3.793482 \\
\hline 乙 & 2.278900 & -1.998468 & 3.36458 \\
\hline & -0.536874 & 0072 & 2.827764 \\
\hline & -0.8550 & 137 & 1.480098 \\
\hline & -2.534916 & 396 & 533 \\
\hline 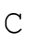 & -2.278900 & 1.998468 & 3.364586 \\
\hline $\mathrm{C}$ & -1.270052 & 1.152398 & 3.793482 \\
\hline$V$ & 0.000000 & 0.000000 & 0.675264 \\
\hline & 1.07 & -1.013092 & 4.851347 \\
\hline $\mathrm{H}$ & 2.905 & -2.5 & 5174 \\
\hline H & -2.90 & 2.5 & 5174 \\
\hline 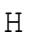 & -1.074391 & 1.013092 & 4.851347 \\
\hline$C$ & 2.534916 & 2.138396 & -1.994533 \\
\hline$C$ & 0.855046 & 0.747137 & -1.480098 \\
\hline C & 0.536874 & 072 & -2.827764 \\
\hline C & 1.2 & 398 & -3.793482 \\
\hline C & 2.278 & 1.998468 & -3.3 \\
\hline C & -0.536874 & -0.480072 & -2.827764 \\
\hline $\mathrm{C}$ & -0.855046 & -0.747137 & -1.480098 \\
\hline C & -2.534916 & -2.138396 & -1.994533 \\
\hline C & -2.278900 & -1.998468 & -3.364586 \\
\hline$C$ & -1.270052 & -1.152398 & -3.793482 \\
\hline $\mathrm{N}$ & 0.000000 & 0.000000 & -0.675264 \\
\hline $\mathrm{H}$ & 1.074391 & 1.013092 & -4.851347 \\
\hline $\mathrm{H}$ & 2.905673 & 2.532653 & -4.065174 \\
\hline $\mathrm{H}$ & 05673 & -2.532653 & -4.065174 \\
\hline $\mathrm{H}$ & -1.074391 & -1.013092 & -4.851347 \\
\hline N & 1.814396 & -1.530489 & 1.041152 \\
\hline & -1.814396 & 1.530489 & 1.041152 \\
\hline N & 1.814396 & 1.530489 & -1.041152 \\
\hline
\end{tabular}

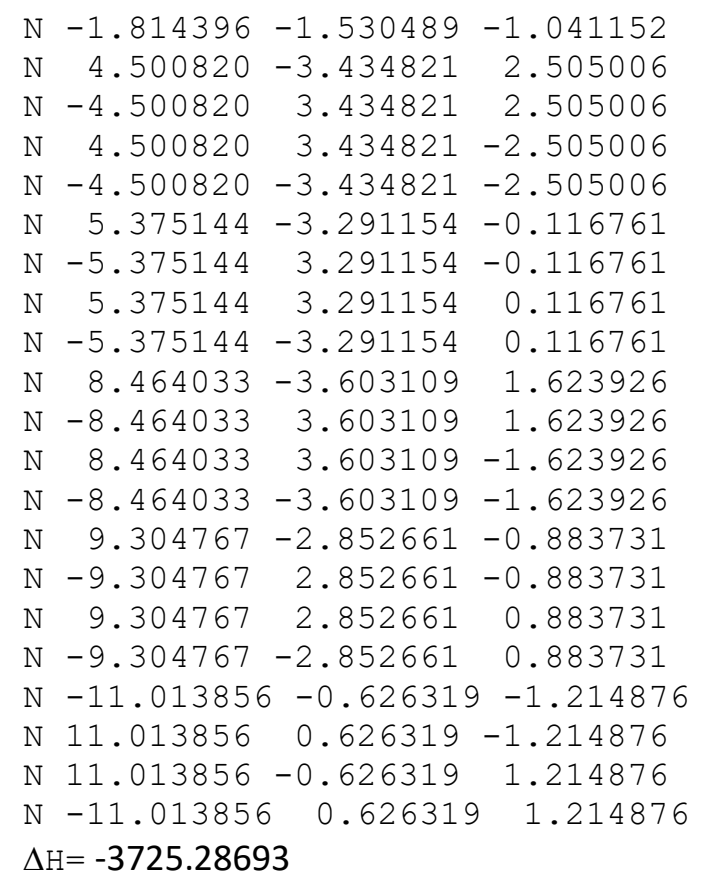

\section{NN- 6}

$\begin{array}{cccc}\text { C } & -7.442460 & 4.262232 & 0.513723 \\ \text { C } & -7.963429 & 4.077893 & -0.771951 \\ \text { C } & -10.041746 & 3.825318 & 0.122907 \\ \text { C } & -9.559799 & 4.294320 & 1.351192 \\ \text { C } & -11.287452 & 3.038364 & 0.025914 \\ \text { C } & -6.016477 & 4.023671 & 0.822525 \\ \text { C } & -5.527216 & 4.147975 & 2.125994 \\ \text { C } & -3.655920 & 2.993372 & 1.541236 \\ \text { C } & -4.069513 & 3.023502 & 0.205229 \\ \text { C } & -12.528124 & 1.498630 & 1.162102 \\ \text { C } & -12.613148 & 0.738012 & -0.013307 \\ \text { C } & -11.716244 & 2.476811 & -1.184957 \\ \text { H } & -7.317830 & 4.054718 & -1.641765 \\ \text { H } & -10.221041 & 4.443230 & 2.196640 \\ \text { H } & -6.112337 & 4.640235 & 2.893738 \\ \text { H } & -3.450786 & 2.596072 & -0.574908 \\ \text { H } & -12.936754 & 1.148845 & 2.102533 \\ \text { H } & -11.468002 & 2.920040 & -2.141782 \\ \text { C } & 7.442460 & -4.262232 & 0.513723 \\ \text { C } & 7.963429 & -4.077893 & -0.771951 \\ \text { C } & 10.041746 & -3.825318 & 0.122907 \\ \text { C } & 9.559799 & -4.294320 & 1.351192 \\ \text { C } & 11.287452 & -3.038364 & 0.025914 \\ \text { C } & 6.016477 & -4.023671 & 0.822525 \\ \text { C } & 5.527216 & -4.147975 & 2.125994 \\ \text { C } & 3.655920 & -2.993372 & 1.541236 \\ \text { C } & 4.069513 & -3.023502 & 0.205229 \\ \text { C } & 12.528124 & -1.498630 & 1.162102 \\ \text { C } & 12.613148 & -0.738012 & -0.013307 \\ \text { C } & 11.716244 & -2.476811 & -1.184957 \\ \text { H } & 7.317830 & -4.054718 & -1.641765\end{array}$




\begin{tabular}{|c|c|c|c|}
\hline & 21041 & -4.443230 & 40 \\
\hline & 6.112337 & -4.640235 & 2.893738 \\
\hline & 3.450786 & -2.596072 & -0.574908 \\
\hline & 12.936754 & -1.148845 & 2.102533 \\
\hline & 11.468002 & -2.920040 & -2.141782 \\
\hline & 7.442460 & 4.262232 & -0.513723 \\
\hline & 7.963429 & 4.077893 & 0.771951 \\
\hline & 10.041746 & 3.825318 & -0.122907 \\
\hline & 9.559799 & 4.294320 & -1.351192 \\
\hline & 11.287452 & 3.038364 & -0.025914 \\
\hline & 6.016477 & 4.023671 & -0.822525 \\
\hline & 5.527216 & 4.147975 & -2.125994 \\
\hline & 3.655920 & 2.993372 & -1.541236 \\
\hline & 4.069513 & 3.023502 & -0.205229 \\
\hline & 12.528124 & 1.498630 & -1.162102 \\
\hline & 12.613148 & 0.738012 & 0.013307 \\
\hline & 11.716244 & 2.476811 & 1.184957 \\
\hline & 7.317830 & 4.054718 & 1.641765 \\
\hline & 10.221041 & 4.443230 & -2.196640 \\
\hline & 6.112337 & 4.640235 & -2.893738 \\
\hline & 3.450786 & 2.596072 & 0.5 \\
\hline & 12.936754 & 1.148845 & -2.102533 \\
\hline & 11.468002 & 2.920040 & 2.141782 \\
\hline & -7.442460 & -4.262232 & -0.513723 \\
\hline & -7.963429 & -4.077893 & 0.771951 \\
\hline & -10.041746 & -3.825318 & -0.122907 \\
\hline & -9.559799 & -4.2 & -1.35119 \\
\hline & -11.287452 & -3.038364 & -0.0259 \\
\hline & -6.016477 & -4.023671 & -0.82252 \\
\hline & -5.527216 & -4.147975 & -2.125994 \\
\hline & -3.655920 & -2.993372 & -1.541236 \\
\hline & -4.069513 & -3.023502 & -0.205229 \\
\hline & -12.528124 & -1.498630 & -1.16210 \\
\hline & -12.613148 & -0.738012 & 0.01330 \\
\hline & -11.716244 & -2.476811 & 1.1849 \\
\hline & -7.317830 & -4.054718 & 1.641765 \\
\hline & 21041 & 3230 & 96640 \\
\hline & -6.112337 & -4 . & -2.8 \\
\hline & -3.450786 & -2.596072 & 0.574908 \\
\hline & -12.936754 & -1.148845 & -2.10253 \\
\hline & -11.468002 & -2.920040 & 2.141782 \\
\hline & 2.492993 & -2.187059 & 1.987716 \\
\hline & 0.843771 & -0.758856 & 1.478849 \\
\hline & 0.530726 & -0.487465 & 2.826940 \\
\hline & 1.256294 & -1.171351 & 3.790444 \\
\hline & 2.246864 & -2.037947 & 3.358517 \\
\hline & -0.530726 & 0.487465 & 2.826940 \\
\hline & -0.843771 & 0.758856 & 1.478849 \\
\hline & -2.492993 & 2.187059 & 1.987716 \\
\hline & -2.246864 & 2.037947 & 3.358517 \\
\hline & -1.256294 & 1.171351 & 3.790444 \\
\hline & 0.000000 & 0.000000 & 0.674845 \\
\hline & 1.066943 & -1.028450 & 4.848964 \\
\hline & 2.861541 & -2.588082 & 4.057512 \\
\hline & -2.861541 & 2.588082 & 4.057512 \\
\hline & -1.066943 & 1.028450 & 4.848964 \\
\hline & 2.492993 & 2.187059 & -1.98771 \\
\hline
\end{tabular}

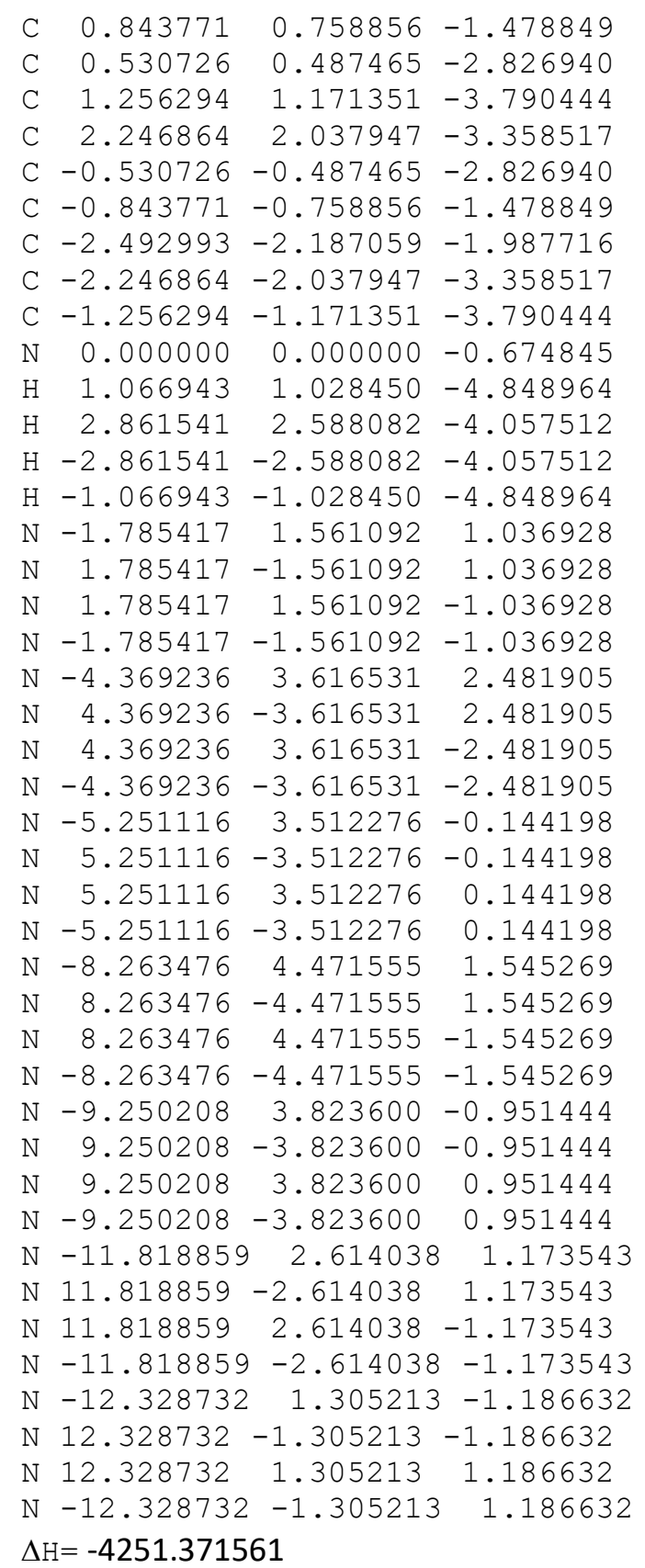

5NN-7

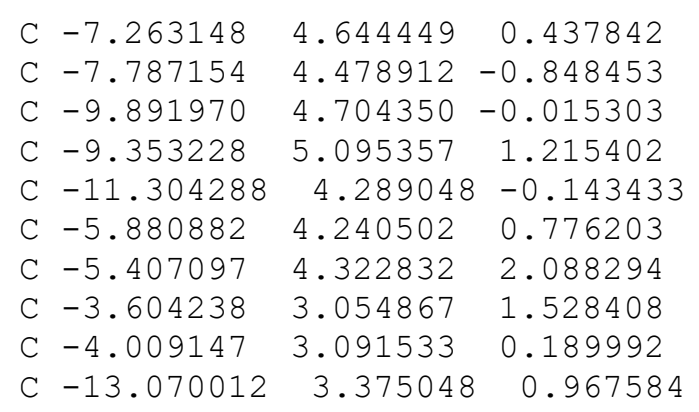




\begin{tabular}{|c|c|c|c|}
\hline $\mathrm{C}$ & -13.391167 & 2.638285 & -0.179919 \\
\hline $\mathrm{C}$ & -11.824930 & 3.806172 & -1.350324 \\
\hline & -7.138457 & 4.278162 & -1.692782 \\
\hline & -9.994510 & 5.392537 & 2.036701 \\
\hline & -5.971087 & 4.852010 & 2.847129 \\
\hline & -3.415093 & 2.612055 & -0.578969 \\
\hline & -13.622765 & 3.253830 & 1.891519 \\
\hline & -11.361372 & 4.042135 & -2.300663 \\
\hline & 7.263148 & -4.644449 & 0.437842 \\
\hline & 7.787154 & -4.478912 & -0.848453 \\
\hline & 9.891970 & -4.704350 & -0.015303 \\
\hline & 9.353228 & -5.095357 & 1.215402 \\
\hline & 11.304288 & -4.289048 & -0.143433 \\
\hline & 5.880882 & -4.240502 & 0.77 \\
\hline & 5.407097 & -4.322832 & 2.088294 \\
\hline & 3.604238 & -3.054867 & 1.52 \\
\hline & 4.009147 & -3.091533 & 0.189992 \\
\hline & 13.070012 & -3.375048 & 0.9 \\
\hline & 13.391167 & -2.638285 & -0.17 \\
\hline & 11.824930 & -3.806172 & -1.35 \\
\hline & 7.138457 & -4.278162 & -1.69 \\
\hline & 9.994510 & -5.392537 & 2.03 \\
\hline & 5.971087 & -4.852010 & 2.8 \\
\hline & 3.415093 & -2.612055 & -0.57 \\
\hline & 13.622765 & -3.253830 & 1.8 \\
\hline & 11.361372 & -4.042135 & $-2 \cdot 30$ \\
\hline & 7.263148 & 4.644449 & -0.4 \\
\hline & 7.787154 & 4.478912 & 0.848453 \\
\hline & 9.891970 & 4.704350 & 0.01 \\
\hline & 9.353228 & 5.095357 & -1.215402 \\
\hline & 11.304288 & 4.289048 & 0.14 \\
\hline & 5.880882 & 4.240502 & -0.776203 \\
\hline & 5.407097 & 4.322832 & -2.08 \\
\hline & 3.604238 & 3.054867 & -1.528408 \\
\hline & 4.009147 & 3.091533 & $-0.1 \xi$ \\
\hline C & 13.070012 & 3.375048 & -0.967584 \\
\hline & 13.391167 & 2.638285 & 0.1 \\
\hline C & 11.824930 & 3.806172 & 1.350324 \\
\hline & 7.138457 & 4.278162 & 1.692 \\
\hline $\mathrm{H}$ & 9.994510 & 5.392537 & -2.036701 \\
\hline $\mathrm{H}$ & 5.971087 & 4.852010 & -2.847129 \\
\hline & 3.415093 & 2.612055 & 0.578969 \\
\hline $\mathrm{H}$ & 13.622765 & 3.253830 & -1.891519 \\
\hline $\mathrm{H}$ & 11.361372 & 4.042135 & 2.300663 \\
\hline $\mathrm{C}$ & -7.263148 & -4.644449 & -0.437842 \\
\hline $\mathrm{C}$ & -7.787154 & -4.478912 & 0.848453 \\
\hline $\mathrm{C}$ & -9.891970 & -4.704350 & 0.015303 \\
\hline $\mathrm{C}$ & -9.353228 & -5.095357 & -1.215402 \\
\hline 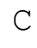 & -11.304288 & -4.289048 & 0.143433 \\
\hline $\mathrm{C}$ & -5.880882 & -4.240502 & -0.776203 \\
\hline $\mathrm{C}$ & -5.407097 & -4.322832 & -2.088294 \\
\hline $\mathrm{C}$ & -3.604238 & -3.054867 & -1.528408 \\
\hline $\mathrm{C}$ & -4.009147 & -3.091533 & -0.189992 \\
\hline $\mathrm{C}$ & -13.070012 & -3.375048 & $3-0.967584$ \\
\hline & -13.391167 & -2.638285 & 79919 \\
\hline & -11.824930 & -3.806172 & 24 \\
\hline & -7.138457 & -4.278162 & \\
\hline
\end{tabular}


$\begin{array}{lrrr}N & 5.148409 & -3.660960 & -0.177442 \\ N & -5.148409 & -3.660960 & 0.177442 \\ N & 8.051123 & 5.034097 & -1.442549 \\ N & -8.051123 & 5.034097 & 1.442549 \\ N & 8.051123 & -5.034097 & 1.442549 \\ N & -8.051123 & -5.034097 & -1.442549 \\ N & 9.094495 & 4.478641 & 1.061314 \\ N & -9.094495 & 4.478641 & -1.061314 \\ N & 9.094495 & -4.478641 & -1.061314 \\ N & -9.094495 & -4.478641 & 1.061314 \\ N & 12.004306 & 4.158405 & -0.984986 \\ N & -12.004306 & 4.158405 & 0.984986 \\ N & 12.004306 & -4.158405 & 0.984986 \\ N & -12.004306 & -4.158405 & -0.984986 \\ N & 12.835293 & 2.951903 & 1.351559 \\ N & -12.835293 & 2.951903 & -1.351559 \\ N & 12.835293 & -2.951903 & -1.351559 \\ N & -12.835293 & -2.951903 & 1.351559 \\ N & 14.195308 & -0.797256 & 1.120909 \\ N & -14.195308 & -0.797256 & -1.120909 \\ N & -14.195308 & 0.797256 & 1.120909 \\ N & 14.195308 & 0.797256 & -1.120909 \\ A & -14777452776 & \end{array}$

\section{$\Delta \mathrm{H}=-4777.452776$}

\section{$5 N N-8$}

\begin{tabular}{|c|c|c|c|}
\hline C & -7.117116 & 4.904304 & 0.379339 \\
\hline C & -7.638611 & 4.759964 & -0.910219 \\
\hline $\mathrm{C}$ & -9.704755 & 5.312783 & -0.138506 \\
\hline $\mathrm{C}$ & -9.145412 & 5.635673 & 1.102022 \\
\hline$C$ & -11.166919 & 5.179378 & $3-0.312254$ \\
\hline $\mathrm{C}$ & -5.779321 & 4.386453 & 0.74 \\
\hline C & -5.324375 & 4.434016 & 2.062569 \\
\hline $\mathrm{C}$ & -3.570009 & 3.093922 & 1.520062 \\
\hline $\mathrm{C}$ & -3.964245 & 3.140662 & 0.178812 \\
\hline $\mathrm{C}$ & -13.157902 & 4.802329 & 0 . \\
\hline C & -13.621245 & 4.146471 & -0.42 \\
\hline C & -11.728983 & 4.765738 & -1.5 \\
\hline $\mathrm{H}$ & -7.006962 & 4.441953 & -1.73 \\
\hline $\mathrm{H}$ & -9.760029 & 6.028898 & 1.9 \\
\hline $\mathrm{H}$ & -5.875348 & 4.984281 & \\
\hline $\mathrm{H}$ & -3.386693 & 2.629794 & -0.582 \\
\hline $\mathrm{H}$ & -13 & & \\
\hline $\mathrm{H}$ & -11.163167 & 4.808442 & -2.4 \\
\hline C & 7.117116 & -4.904304 & \\
\hline $\mathrm{C}$ & 7.638611 & -4.759964 & -0.91 \\
\hline $\mathrm{C}$ & 9.704755 & -5.312783 & -0 . \\
\hline C & 9.145412 & -5.635673 & 1.10 \\
\hline C & 11.166919 & -5.179378 & -0.31 \\
\hline $\mathrm{C}$ & 5.779321 & -4.386453 & 0.74 \\
\hline $\mathrm{C}$ & 5.324375 & -4.434016 & 2.0 \\
\hline $\mathrm{C}$ & 3.570009 & -3.093922 & 1.52 \\
\hline $\mathrm{C}$ & 3.964245 & -3.140662 & 0.1 \\
\hline $\mathrm{C}$ & 13.157902 & -4.802329 & 0.7230 \\
\hline $\mathrm{C}$ & 13.621245 & -4.146471 & -0.42 \\
\hline C & 11.728983 & -4.765738 & -1.52 \\
\hline
\end{tabular}

\begin{tabular}{|c|c|c|c|}
\hline $\mathrm{H}$ & 7.006962 & -4.441953 & -1.731179 \\
\hline $\mathrm{H}$ & 9.760029 & -6.028898 & 1.9 \\
\hline & 5.875348 & -4.984281 & 2.815878 \\
\hline $\mathrm{H}$ & 3.386693 & -2.629794 & -0.582449 \\
\hline F & 13.766029 & -4.865705 & 1.617536 \\
\hline $\mathrm{H}$ & 11.163167 & -4.808442 & -2.448166 \\
\hline $\mathrm{C}$ & 7.117116 & 4.904304 & -0.37 \\
\hline$c$ & 7.638611 & 4.759964 & 0.910219 \\
\hline $\mathrm{C}$ & 9.704755 & 5.312783 & 0.138506 \\
\hline $\mathrm{C}$ & 9.145412 & 5.635673 & -1.102022 \\
\hline C & 11.166919 & 5.179378 & 0.312254 \\
\hline $\mathrm{C}$ & 5.779321 & 4.386453 & -0.742 \\
\hline C & 5.324375 & 4.434016 & -2.06 \\
\hline $\mathrm{C}$ & 3.570009 & 3.093922 & -1.520062 \\
\hline 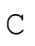 & 3.964245 & 3.140662 & -0.178812 \\
\hline c & 13.157902 & 4.802329 & -0.72 \\
\hline 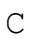 & 13.621245 & 4.146471 & 0.4 \\
\hline $\mathrm{C}$ & 11.728983 & 4.765738 & 1.5252 \\
\hline $\mathrm{H}$ & 7.00 & 4.44 & 1.7 \\
\hline $\mathrm{H}$ & 9.760029 & 6.02 & $-1 \cdot 9$ \\
\hline $\mathrm{H}$ & 5.87 & 4.98 & -2.8 \\
\hline $\mathrm{H}$ & 3.386693 & 2.629 & 0.5 \\
\hline $\mathrm{H}$ & 13.76 & 4.86 & $-1 \cdot 6$ \\
\hline $\mathrm{H}$ & 11.16 & 4.80 & 2.4 \\
\hline $\mathrm{C}$ & -7.11 & -4.90 & -0.3 \\
\hline $\mathrm{C}$ & -7.63 & -4.75 & 0.9 \\
\hline $\mathrm{C}$ & -9.70 & -5.31 & 0.1 \\
\hline C & -9.145412 & -5.63 & -1.1 \\
\hline $\mathrm{C}$ & $-11 \cdot 1$ & -5.1 & 254 \\
\hline C & -5.7 & $-4 \cdot 38$ & -0.7 \\
\hline $\mathrm{C}$ & -5.32 & -4.43 & -2.0 \\
\hline $\mathrm{C}$ & -3.57 & -3.09 & -1.5 \\
\hline C & -3.96 & -3.14 & $-0.17 \varepsilon$ \\
\hline $\mathrm{C}$ & -13.1 & -4.8 & -0 \\
\hline C & $-13 \cdot 621245$ & -4.1 & 611 \\
\hline $\mathrm{C}$ & $-11 \cdot 7$ & -4 & \\
\hline $\mathrm{H}$ & -7.006962 & -4.441953 & 1.731179 \\
\hline $\mathrm{H}$ & -9.76 & -6.02 & $-1 \cdot 9$ \\
\hline $\mathrm{H}$ & -5.875348 & -4.984281 & -2.815878 \\
\hline $\mathrm{H}$ & -3.38 & -2.629 & 0.5 \\
\hline $\mathrm{H}$ & -13.766029 & -4.865705 & -1.61 \\
\hline $\mathrm{H}$ & -11.163167 & -4.8 & 166 \\
\hline C & 2.454743 & -2.229814 & 1.979206 \\
\hline $\mathrm{C}$ & 0.83 & -0.76 & $1.47^{\circ}$ \\
\hline $\mathrm{C}$ & 0.526212 & -0.49 & 2.82624 \\
\hline $\mathrm{C}$ & 1.24 & -1.18 & 3.7 \\
\hline C & 2.222533 & -2.069 & 3.350974 \\
\hline $\mathrm{C}$ & -0.526212 & 0.45 & 2.82 \\
\hline $\mathrm{C}$ & -0.833568 & 0.768 & 1.477578 \\
\hline $\mathrm{C}$ & -2.45 & 2.22 & 1.979206 \\
\hline C & -2.222533 & 2.069443 & 3.350974 \\
\hline $\mathrm{C}$ & -1.248 & 1.185015 & 3.786757 \\
\hline $\mathrm{N}$ & 0.000000 & 0.000000 & 0.674454 \\
\hline $\mathrm{H}$ & 1.067461 & -1.038130 & 4.846130 \\
\hline $\mathrm{H}$ & 2.827812 & -2.632373 & 4.048029 \\
\hline $\mathrm{H}$ & -2.827812 & 2.63 & 4.048029 \\
\hline 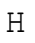 & -1.067461 & 1.038130 & 4.846130 \\
\hline
\end{tabular}




\begin{tabular}{|c|c|c|c|}
\hline C & 2.454743 & 2.229814 & -1.979206 \\
\hline C & 0.833568 & 0.768595 & -1.477578 \\
\hline & 0.526212 & 0.493146 & -2.826242 \\
\hline $\mathrm{C}$ & 1.248707 & 1.185015 & -3.786757 \\
\hline C & 2.222533 & 2.069443 & -3.350974 \\
\hline & -0.526212 & -0.493146 & -2.826242 \\
\hline C & -0.833568 & -0.768595 & -1.477578 \\
\hline & -2.454743 & -2.229814 & -1.979206 \\
\hline$\checkmark$ & -2.222533 & -2.069443 & -3.350974 \\
\hline & -1.248707 & -1.185015 & -3.786757 \\
\hline$V$ & 0.000000 & 0.000000 & -0.674454 \\
\hline & 1.067461 & 1.038130 & -4.846130 \\
\hline $\mathrm{H}$ & 2.827812 & 2.632373 & -4.048029 \\
\hline & -2.827812 & -2.632373 & -4.048029 \\
\hline $\mathrm{H}$ & -1.067461 & -1.038130 & -4.846130 \\
\hline$\checkmark$ & -14.705614 & $4-3.145287$ & 0.357780 \\
\hline$C$ & -15.795836 & $6-0.732379$ & 787 \\
\hline C & 15.795836 & 0.732379 & 0.094787 \\
\hline C & 14.70 & 3.145287 & 0.35 \\
\hline $\mathrm{C}$ & -15.083833 & $3-2.395183$ & 1.478490 \\
\hline C & -15.7 & $4-1.614442$ & -0 . \\
\hline C & 15.717884 & 1.614442 & -0.990735 \\
\hline C & 15.08 & 2.395183 & 1.47 \\
\hline $\mathrm{H}$ & -16.0 & $1-1.322892$ & -1.98 \\
\hline $\mathrm{H}$ & -14.8 & $2-2.746759$ & 2.4 \\
\hline $\mathrm{H}$ & 16. & 1.322 & -1.98 \\
\hline $\mathrm{H}$ & 14.89 & 2.746759 & 2.48 \\
\hline C & -14.7 & $4 \quad 3.145287$ & -0 \\
\hline C & 14.70 & -3.14 & -0.357780 \\
\hline $\mathrm{C}$ & -15.7 & 379 & $-0.0 s$ \\
\hline $\mathrm{C}$ & 15.75 & -0.732 & -0.094787 \\
\hline C & -15.0 & 32.3 & $3-1 \cdot 4$ \\
\hline $\mathrm{C}$ & 15.08 & $-2 \cdot 3$ & -1.478490 \\
\hline C & -15.7 & 41.61 & 0.9 \\
\hline $\mathrm{C}$ & 15.71 & -1.61 & 0.990735 \\
\hline $\mathrm{H}$ & -14.8 & $2 \quad 2.74$ & -2.4 \\
\hline $\mathrm{H}$ & 14.892902 & -2.746759 & -2.485259 \\
\hline $\mathrm{H}$ & 16.04 & -1.322892 & 1.981718 \\
\hline $\mathrm{H}$ & -16.0 & 1.322892 & 1.981718 \\
\hline $\mathrm{N}$ & 1.75 & 1.588 & -1.031871 \\
\hline N & 1.757251 & -1.588471 & 1.031871 \\
\hline $\mathrm{N}$ & -1.757251 & 1.588471 & 1.031871 \\
\hline $\mathrm{N}$ & -1.757251 & -1.588471 & -1.031871 \\
\hline $\mathrm{N}$ & 4.240596 & 3.779600 & -2.448184 \\
\hline $\mathrm{N}$ & -4.240596 & 3.779600 & 2.448184 \\
\hline $\mathrm{N}$ & 4.240596 & -3.779600 & 2.448184 \\
\hline $\mathrm{N}$ & -4.240596 & -3.779600 & -2.448184 \\
\hline $\mathrm{N}$ & 5.069284 & 3.765741 & 0.202334 \\
\hline $\mathrm{N}$ & -5.069284 & 3.765741 & -0.202334 \\
\hline $\mathrm{N}$ & 5.069284 & -3.765741 & -0.202334 \\
\hline $\mathrm{N}$ & -5.069284 & -3.765741 & 0.202334 \\
\hline $\mathrm{N}$ & 7.867839 & 5.407819 & -1.361691 \\
\hline $\mathrm{N}$ & -7.867839 & 5.407819 & 1.361691 \\
\hline $\mathrm{N}$ & 7.867839 & -5.407819 & 1.361691 \\
\hline $\mathrm{N}$ & -7.867839 & -5.407819 & -1.361691 \\
\hline $\mathrm{N}$ & 8.927957 & 4.938295 & 1.157092 \\
\hline & -8.927957 & 4.938295 & -1.157092 \\
\hline
\end{tabular}

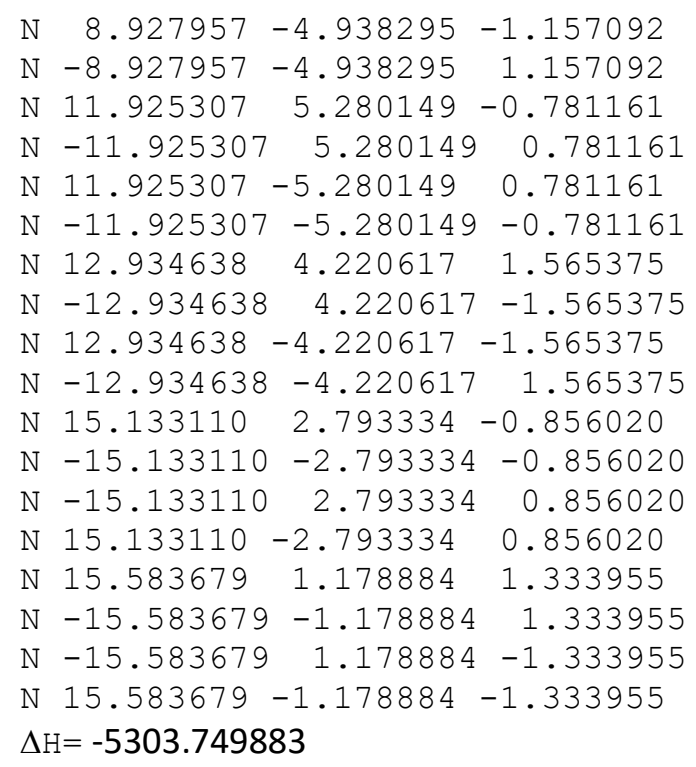

5CC- 4 


\begin{tabular}{|c|c|c|c|}
\hline $\mathrm{H}$ & 545 & 932 & -3.416550 \\
\hline $\mathrm{H}$ & 4.095684 & 1.958732 & 0.267477 \\
\hline & -8.322300 & -2.437786 & -0.955994 \\
\hline & -8.806926 & -2.452337 & 0.361841 \\
\hline & -10.17 & $5-0.708363$ & $3-0.21175$ \\
\hline & -10.0 & $4-1.080125$ & $5-1.565302$ \\
\hline & -6.890454 & -2.594872 & -1.275196 \\
\hline & 24926 & -2.620409 & -2.593025 \\
\hline & -4 . & -1 & -1.8 \\
\hline & 840 & -2.125238 & -0.55486 \\
\hline & -8. & -3.095540 & 1.13291 \\
\hline & -10 & $4-0.628455$ & $5-2.35365$ \\
\hline & -7.088545 & -2.858932 & -3.416550 \\
\hline & -4 & -1 & \\
\hline & 990 & -1.3 & 2 . \\
\hline & 525 & -0. & \\
\hline & 0.674706 & -0.267625 & 2.913 \\
\hline & 1.48 & -0.717 & \\
\hline 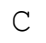 & 2.7 & -1.2 & \\
\hline & -0.6 & 0. & \\
\hline & -1.1 & 0 . & 1 . \\
\hline & -3.051990 & 1.36 & 2. \\
\hline 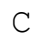 & -2.704828 & 1.269602 & 3.5 \\
\hline & -1.48 & 0.7 & \\
\hline $\mathrm{H}$ & 1.17 & -0 & \\
\hline $\mathrm{H}$ & 3.3 & -1.6 & \\
\hline $\mathrm{H}$ & -3.39 & 1.6 & 4. \\
\hline $\mathrm{H}$ & -1.174815 & 0.66 & 4. \\
\hline $\mathrm{C}$ & 3.051990 & 1.366874 & -2.2 \\
\hline 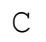 & 1.1 & 0.3 & -1.582640 \\
\hline C & 0.6 & 5 & -2 . \\
\hline C & 12 & & -3 \\
\hline 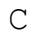 & 2.70 & 1.2 & -3 \\
\hline $\mathrm{C}$ & -0.674706 & -0.267625 & -2.9 \\
\hline $\mathrm{C}$ & -1.119525 & -0.37 & -1.58264 \\
\hline c & -3.051990 & -1.366874 & -2.236648 \\
\hline 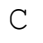 & -2.7 & -1.2 & -3. \\
\hline C & -1.2 & -0. & -3 \\
\hline $\mathrm{H}$ & 1.1 & 79 & -4 \\
\hline $\mathrm{H}$ & 3.394340 & 1.646666 & -4.331946 \\
\hline 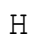 & -3.394340 & -1.64 & -4.33 \\
\hline $\mathrm{H}$ & -1.17 & -0.664279 & -4.977 \\
\hline 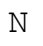 & -2.272 & -0.9 & -1.23 \\
\hline $\mathrm{N}$ & 2 . & -0.9 & 1 . \\
\hline & -2.2 & 0 . & \\
\hline $\mathbb{N}$ & 2.272067 & 399 & -1.23 \\
\hline $\mathrm{N}$ & -5.180452 & -2.286220 & -2.885698 \\
\hline N & -5.180452 & 2.286220 & 2.885698 \\
\hline $\mathrm{N}$ & 5.180452 & -2.286220 & 2. \\
\hline $\mathrm{N}$ & 0452 & 2.286220 & -2.8 \\
\hline & -6.041138 & -2.419507 & -0 \\
\hline $\mathrm{IN}$ & -6.041138 & 2.419507 & \\
\hline $\mathrm{N}$ & 6.041138 & -2.419507 & 0.2 \\
\hline $\mathbb{N}$ & 6.041138 & 2.419507 & -0.259445 \\
\hline $\mathrm{N}$ & -9.080328 & -1.893380 & -1.908994 \\
\hline & -9.080328 & 1.8 & 1.9 \\
\hline & 9.080 & -1.893380 & 1.90899 \\
\hline
\end{tabular}

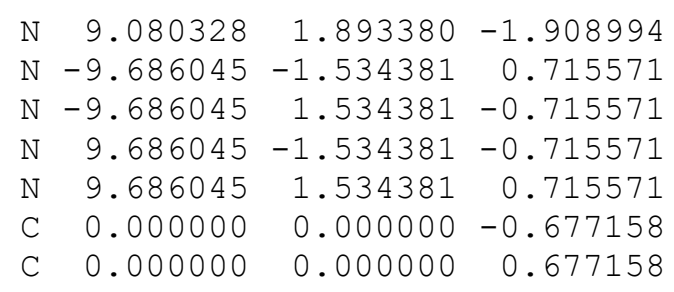

$\Delta \mathrm{H}=-3165.873901$

\section{CC- 5}




\begin{tabular}{|c|c|c|c|}
\hline C & -10.334488 & -2.751344 & -1.756092 \\
\hline $\mathrm{C}$ & -11.716137 & -1.298296 & $5-0.231783$ \\
\hline C & -6.793730 & -2.885108 & -1.234674 \\
\hline$C$ & -6.306286 & -2.914621 & -2.543549 \\
\hline$C$ & -4.353451 & -1.975077 & -1.849078 \\
\hline$C$ & -4.797546 & -2.054610 & -0.525869 \\
\hline C & -11.992529 & 0.816298 & $3-1.061167$ \\
\hline $\mathrm{C}$ & -11.716137 & 1.298296 & 0.231783 \\
\hline C & -11.992529 & -0.816298 & 1.061167 \\
\hline $\mathrm{H}$ & -8.113627 & -3.313953 & 1.212555 \\
\hline $\mathrm{H}$ & -11.003072 & -2.698819 & -2.607402 \\
\hline $\mathrm{H}$ & -6.921229 & -3.284960 & -3.355452 \\
\hline $\mathrm{H}$ & -4.163737 & -1.721296 & 0.288085 \\
\hline $\mathrm{H}$ & -12.130432 & 1.464375 & $5-1.917972$ \\
\hline $\mathrm{H}$ & -12.130432 & -1.464375 & 1.917972 \\
\hline $\mathrm{C}$ & 3.061450 & -1.344923 & 2.221507 \\
\hline C & 1.122055 & -0.359622 & 1.579507 \\
\hline 乙 & 0.679309 & -0.258230 & 2.911501 \\
\hline$\tau$ & 1.503307 & -0.695806 & 3.932517 \\
\hline $\mathrm{C}$ & 2.723919 & -1.241487 & 3.576037 \\
\hline $\mathrm{C}$ & -0.679309 & 0.258230 & 2.91 \\
\hline $\mathrm{C}$ & -1.122055 & 0.359622 & 1.579507 \\
\hline $\mathrm{C}$ & -3.06 & 1.344923 & 2.22 \\
\hline $\mathrm{C}$ & -2.723919 & 1.241487 & 3.57 \\
\hline $\mathrm{C}$ & -1.503 & 0.695806 & 3.93 \\
\hline $\mathrm{H}$ & 1.199974 & -0.641326 & 4.972947 \\
\hline $\mathrm{H}$ & 3.416 & -1.619 & 4.31 \\
\hline $\mathrm{H}$ & -3.41 & 1.619 & 4.31 \\
\hline $\mathrm{H}$ & -1.199974 & 0.641326 & 4.972947 \\
\hline $\mathrm{C}$ & 3.0 & 1.344923 & -2.22 \\
\hline C & 1.122 & 0.35 & -1.579507 \\
\hline $\mathrm{C}$ & 0.67 & 0.25 & $-2 \cdot 91$ \\
\hline $\mathrm{C}$ & 1.503 & 0.69 & -3.932517 \\
\hline C & 2.72 & 1.24 & -3.5 \\
\hline C & -0.679309 & -0.258230 & -2.911501 \\
\hline C & -1.122 & -0.359 & $-1 \cdot 5$ \\
\hline C & -3.061450 & -1.344923 & -2.221507 \\
\hline $\mathrm{C}$ & -2.72 & -1.241487 & -3.576037 \\
\hline C & -1.503307 & -0.695806 & -3.932517 \\
\hline $\mathrm{H}$ & 1.199974 & 0.641326 & -4.972947 \\
\hline $\mathrm{H}$ & 3.416463 & 1.619018 & -4.315906 \\
\hline $\mathrm{H}$ & -3.416463 & -1.619018 & -4.315906 \\
\hline $\mathrm{H}$ & -1.199974 & -0.641326 & -4.972947 \\
\hline $\mathrm{N}$ & 2.275701 & -0.909993 & 1.229852 \\
\hline $\mathrm{N}$ & -2.275701 & 0.909993 & 1.229852 \\
\hline $\mathrm{N}$ & 2.275701 & 0.909993 & -1.229852 \\
\hline $\mathrm{N}$ & -2.275701 & -0.909993 & -1.229852 \\
\hline $\mathrm{N}$ & 5.105179 & -2.451718 & 2.845187 \\
\hline $\mathrm{N}$ & -5.105179 & 2.451718 & 2.845187 \\
\hline $\mathrm{N}$ & 5.105179 & 2.451718 & -2.845187 \\
\hline $\mathrm{N}$ & -5.105179 & -2.451718 & -2.845187 \\
\hline $\mathrm{N}$ & 6.013494 & -2.492089 & 0.224513 \\
\hline $\mathrm{N}$ & -6.013494 & 2.492089 & 0.224513 \\
\hline $\mathrm{N}$ & 6.013494 & 2.492089 & -0.224513 \\
\hline $\mathrm{N}$ & -6.0 & -2.492089 & -0.224513 \\
\hline $\mathrm{N}$ & 9.0 & -3.051036 & 1.984902 \\
\hline $\mathrm{N}$ & -9.068196 & 3.051036 & 1.984902 \\
\hline
\end{tabular}

$\begin{array}{crrr}\mathrm{N} & 9.068196 & 3.051036 & -1.984902 \\ \mathrm{~N} & -9.068196 & -3.051036 & -1.984902 \\ \mathrm{~N} & 9.979692 & -2.693862 & -0.582544 \\ \mathrm{~N} & -9.979692 & 2.693862 & -0.582544 \\ \mathrm{~N} & 9.979692 & 2.693862 & 0.582544 \\ \mathrm{~N} & -9.979692 & -2.693862 & 0.582544 \\ \mathrm{~N} & -11.913474 & -0.483623 & -1.268436 \\ \mathrm{~N} & 11.913474 & 0.483623 & -1.268436 \\ \mathrm{~N} & 11.913474 & -0.483623 & 1.268436 \\ \mathrm{~N} & -11.913474 & 0.483623 & 1.268436 \\ \mathrm{C} & 0.000000 & 0.000000 & 0.676242 \\ \mathrm{C} & 0.000000 & 0.000000 & -0.676242\end{array}$

\section{$\Delta \mathrm{H}=-3691.960965$}

\section{$5 \mathrm{CC}-6$}

$\begin{array}{lccc}\mathrm{C} & -8.125677 & 3.396155 & 0.866081 \\ \mathrm{C} & -8.641099 & 3.362192 & -0.433453 \\ \mathrm{C} & -10.753721 & 3.419046 & 0.413067 \\ \mathrm{C} & -10.229294 & 3.716083 & 1.676782 \\ \mathrm{C} & -12.113251 & 2.868973 & 0.246525 \\ \mathrm{C} & -6.737009 & 2.990230 & 1.173540 \\ \mathrm{C} & -6.264327 & 3.000305 & 2.487858 \\ \mathrm{C} & -4.363609 & 1.940859 & 1.828681 \\ \mathrm{C} & -4.808265 & 1.989196 & 0.503637 \\ \mathrm{C} & -13.553311 & 1.415632 & 1.261409 \\ \mathrm{C} & -13.671226 & 0.737124 & 0.036802 \\ \mathrm{C} & -12.603705 & 2.466245 & -1.004999 \\ \mathrm{H} & -7.986541 & 3.277163 & -1.292819 \\ \mathrm{H} & -10.877749 & 3.915313 & 2.521940 \\ \mathrm{H} & -6.863449 & 3.423720 & 3.285166 \\ \mathrm{H} & -4.196815 & 1.584056 & -0.294911 \\ \mathrm{H} & -14.024015 & 1.060873 & 2.170366 \\ \mathrm{H} & -12.312188 & 2.954711 & -1.926814 \\ \mathrm{C} & 8.125677 & -3.396155 & 0.866081 \\ \mathrm{C} & 8.641099 & -3.362192 & -0.433453 \\ \mathrm{C} & 10.753721 & -3.419046 & 0.413067 \\ \mathrm{C} & 10.229294 & -3.716083 & 1.676782 \\ \mathrm{C} & 12.113251 & -2.868973 & 0.246525 \\ \mathrm{C} & 6.737009 & -2.990230 & 1.173540 \\ \mathrm{C} & 6.264327 & -3.000305 & 2.487858 \\ \mathrm{C} & 4.363609 & -1.940859 & 1.828681 \\ \mathrm{C} & 4.808265 & -1.989196 & 0.503637 \\ \mathrm{C} & 13.553311 & -1.415632 & 1.261409 \\ \mathrm{C} & 13.671226 & -0.737124 & 0.036802 \\ \mathrm{C} & 12.603705 & -2.466245 & -1.004999 \\ \mathrm{H} & 7.986541 & -3.277163 & -1.292819 \\ \mathrm{H} & 10.877749 & -3.915313 & 2.521940 \\ \mathrm{H} & 6.863449 & -3.423720 & 3.285166 \\ \mathrm{H} & 4.196815 & -1.584056 & -0.294911 \\ \mathrm{H} & 14.024015 & -1.060873 & 2.170366 \\ \mathrm{H} & 12.312188 & -2.954711 & -1.926814 \\ \mathrm{C} & 8.125677 & 3.396155 & -0.866081 \\ \mathrm{C} & 8.641099 & 3.362192 & 0.433453 \\ \mathrm{C} & 10.753721 & 3.419046 & -0.413067 \\ \mathrm{C} & 10.229294 & 3.716083 & -1.676782\end{array}$




\begin{tabular}{|c|c|c|c|}
\hline C & 12.113251 & 2.868973 & -0.246525 \\
\hline $\mathrm{C}$ & 6.737009 & 2.990230 & -1.173540 \\
\hline $\mathrm{C}$ & 6.264327 & 3.000305 & -2.487858 \\
\hline C & 4.363609 & 1.940859 & -1.828681 \\
\hline C & 4.808265 & 1.989196 & -0.503637 \\
\hline $\mathrm{C}$ & 13.553311 & 1.415632 & -1.261409 \\
\hline$c$ & 13.671226 & 0.737124 & -0.036802 \\
\hline C & 12.603705 & 2.466245 & 1.004999 \\
\hline $\mathrm{H}$ & 7.986541 & 3.277163 & 1.292819 \\
\hline $\mathrm{H}$ & 10.877749 & 3.915313 & -2.521940 \\
\hline $\mathrm{H}$ & 6.863449 & 3.423720 & -3.285166 \\
\hline $\mathrm{H}$ & 4.196815 & 1.584056 & 0.294911 \\
\hline $\mathrm{H}$ & 14.024015 & 1.060873 & -2.170366 \\
\hline $\mathrm{H}$ & 12.312188 & 2.954711 & 1.926814 \\
\hline C & -8.125677 & -3.396155 & -0.866081 \\
\hline C & -8.641099 & -3.362192 & 0.433453 \\
\hline C & -10.753721 & -3.419046 & $5-0.4$ \\
\hline C & -10.229294 & -3.716083 & $3-1.67$ \\
\hline C & $-12 \cdot 1$ & -2.868973 & $3-0.2$ \\
\hline C & -6.73 & -2.990230 & -1.173540 \\
\hline C & -6.26 & -3.000305 & $-2.4 \varepsilon$ \\
\hline $\mathrm{C}$ & -4.363609 & -1.940859 & -1.828681 \\
\hline C & -4.80 & -1.989196 & -0.5 \\
\hline C & -13.553311 & -1.415632 & -1.26 \\
\hline C & -13.671226 & -0.737124 & -0 \\
\hline $\mathrm{C}$ & -12.603705 & -2.466245 & 1.0 \\
\hline $\mathrm{H}$ & -7.98 & -3.277163 & 1.292 \\
\hline $\mathrm{H}$ & -10.8 & -3.91 & $3-2$. \\
\hline $\mathrm{H}$ & -6.86 & -3.423720 & -3.285 \\
\hline $\mathrm{H}$ & -4 & -1.58 & 0.2 \\
\hline $\mathrm{H}$ & $-14 \cdot 0$ & -1.060873 & $3-2.1$ \\
\hline $\mathrm{H}$ & $-12 \cdot 312188$ & -2.95 & \\
\hline $\mathrm{C}$ & 3.07 & -1.302780 & 2.21 \\
\hline $\mathrm{C}$ & 1.12 & -0.34 & \\
\hline $\mathrm{C}$ & 0.684249 & -0.246772 & 2.91 \\
\hline $\mathrm{C}$ & 1.52 & -0.665 & \\
\hline $\mathrm{C}$ & 2.749246 & -1.194874 & 3.569849 \\
\hline $\mathrm{C}$ & -0.684249 & 0.246772 & 2.91 \\
\hline $\mathrm{C}$ & -1.125186 & 0.344987 & 1.57 \\
\hline $\mathrm{C}$ & -3.07 & 1.302780 & 2.2 \\
\hline $\mathrm{C}$ & -2.749246 & 1.194874 & 3.569849 \\
\hline $\mathrm{C}$ & -1.521717 & 0.665584 & 3.92 \\
\hline $\mathrm{H}$ & 1.223741 & -0.610598 & 4.971909 \\
\hline $\mathrm{H}$ & 3.449442 & -1.560943 & 4.308411 \\
\hline $\mathrm{H}$ & -3.449442 & 1.560943 & 4.30841 \\
\hline $\mathrm{H}$ & -1.223741 & 0.610598 & 4.971 \\
\hline $\mathrm{C}$ & 3.079661 & 1.302780 & -2.213983 \\
\hline $\mathrm{C}$ & 1.125186 & 0.344987 & -1.578592 \\
\hline $\mathrm{C}$ & 0.684249 & 0.246772 & -2.91144 \\
\hline $\mathrm{C}$ & 1.521717 & 0.665584 & -3.929 \\
\hline $\mathrm{C}$ & 2.749246 & 1.194874 & -3.569849 \\
\hline $\mathrm{C}$ & -0.684249 & -0.246772 & -2.911443 \\
\hline $\mathrm{C}$ & -1.125186 & -0.344987 & -1.578592 \\
\hline $\mathrm{C}$ & -3.079661 & -1.302780 & -2.213983 \\
\hline $\mathrm{C}$ & -2.749246 & -1.194874 & -3.569849 \\
\hline $\mathrm{C}$ & -1.521717 & -0.665584 & -3.929969 \\
\hline$H$ & 1.223741 & 0.610598 & -4.971909 \\
\hline
\end{tabular}

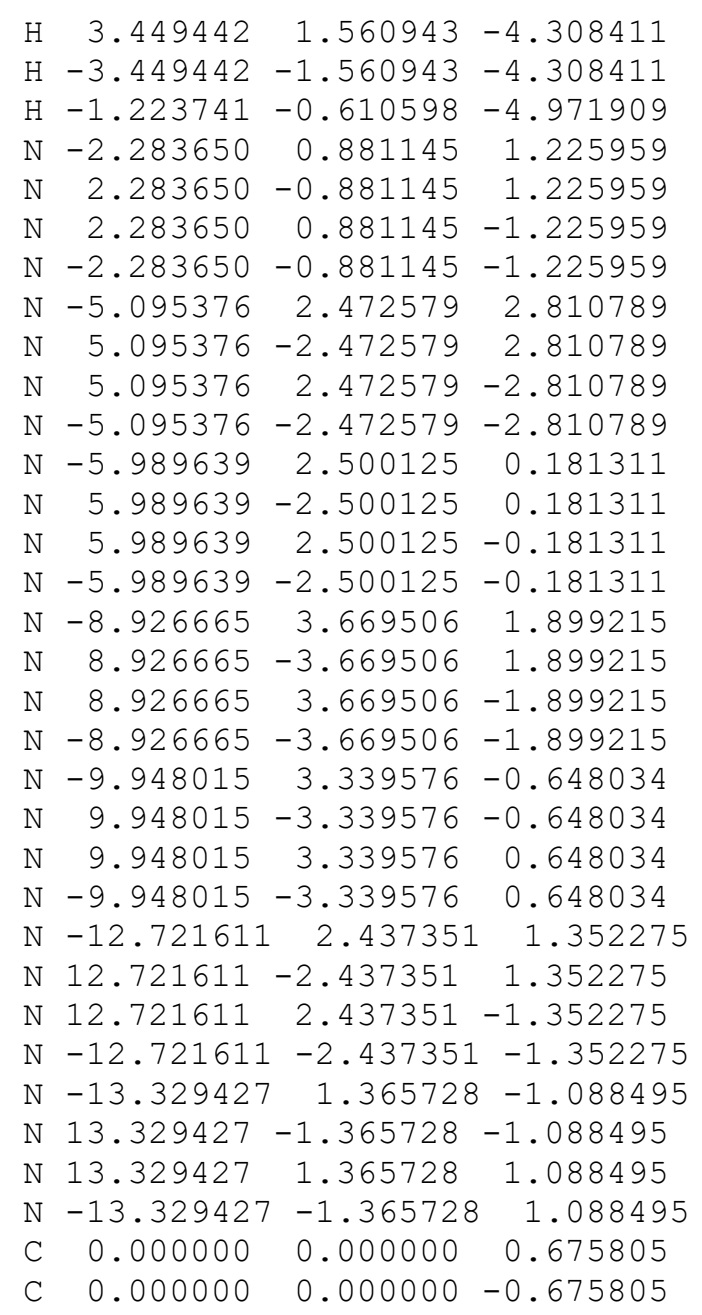

$\Delta \mathrm{H}=-4218.04536$

\section{$5 \mathrm{CC}-7$}

$\begin{array}{llcc}\mathrm{C}-8.067228 & 3.503585 & 0.784572 \\ \mathrm{C}-8.583544 & 3.448283 & -0.513381 \\ \mathrm{C}-10.648335 & 3.974313 & 0.282583 \\ \mathrm{C}-10.091946 & 4.209504 & 1.544135 \\ \mathrm{C}-12.107793 & 3.835599 & 0.099320 \\ \mathrm{C}-6.722782 & 2.983391 & 1.120087 \\ \mathrm{C}-6.274577 & 2.974177 & 2.442945 \\ \mathrm{C}-4.389573 & 1.871798 & 1.814358 \\ \mathrm{C}-4.825139 & 1.905171 & 0.485487 \\ \mathrm{C}-14.040612 & 3.199951 & 1.126764 \\ \mathrm{C}-14.436468 & 2.561589 & -0.057235 \\ \mathrm{C}-12.671283 & 3.512049 & -1.141271 \\ \mathrm{H}-7.951026 & 3.177595 & -1.350638 \\ \mathrm{H}-10.706092 & 4.555811 & 2.366999 \\ \mathrm{H}-6.874681 & 3.415956 & 3.229307 \\ \mathrm{H}-4.218398 & 1.469584 & -0.300684 \\ \mathrm{H}-14.635268 & 3.144215 & 2.030792 \\ \mathrm{H}-12.152828 & 3.713440 & -2.070922 \\ \mathrm{C} & 8.067228 & -3.503585 & 0.784572\end{array}$




\begin{tabular}{|c|c|c|c|}
\hline $\mathrm{C}$ & 8.583544 & -3.448283 & -0.513381 \\
\hline $\mathrm{C}$ & 10.648335 & -3.974313 & 0.282583 \\
\hline$C$ & 10.091946 & -4.209504 & 1.544135 \\
\hline & 12.107793 & -3.835599 & 0.099320 \\
\hline C & 6.722782 & -2.983391 & 1.120087 \\
\hline & 6.274577 & -2.974177 & 2.442945 \\
\hline & 4.389573 & -1.871798 & 1.814358 \\
\hline & 4.825139 & -1.905171 & 0.485487 \\
\hline & 14.040612 & -3.199951 & 1.126764 \\
\hline & 14.436468 & -2.561589 & -0.057235 \\
\hline & 12.671283 & -3.512049 & -1.141271 \\
\hline & 7.951026 & -3.177595 & -1.350638 \\
\hline & 10.706092 & -4.555811 & 2.366999 \\
\hline & 6.874681 & -3.415956 & 3.229307 \\
\hline & 4.218398 & -1.469584 & -0.300684 \\
\hline & 14.635268 & -3.144215 & 2.030792 \\
\hline & 12.152828 & -3.713440 & -2.070922 \\
\hline & 8.067228 & 3.503585 & -0.784572 \\
\hline & 8.583544 & 3.448283 & 0.51 \\
\hline & 10.648335 & 3.974313 & -0.282583 \\
\hline & 10.09 & 4.209504 & -1.5 \\
\hline & 12.107793 & 3.835599 & -0.099320 \\
\hline & 6.722782 & 2.983391 & -1.12 \\
\hline & 6.274577 & 2.974177 & -2.442945 \\
\hline & 4.389573 & 1.871798 & -1.81 \\
\hline & 4.82 & 1.90 & -0.4 \\
\hline & 14.040612 & 3.199951 & $-1 \cdot 12$ \\
\hline C & 14.43 & 2.56 & 0.0 \\
\hline 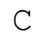 & 12.67 & 3.512049 & 1.14 \\
\hline & 7.95 & 3.17 & 1.3 \\
\hline & 10.70 & 4.555811 & $-2 \cdot 36$ \\
\hline & 6.87 & 3.41 & $-3 \cdot 2$ \\
\hline 11 & 4.218398 & 1.469584 & 0.3 \\
\hline & 14.63 & 3.144215 & $-2 \cdot 0$ \\
\hline & 12.152828 & 3.713440 & 2.07 \\
\hline & -8.06 & -3.503585 & -0.7 \\
\hline 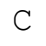 & -8.583544 & -3.448283 & 0.513381 \\
\hline & -10.648335 & $5-3.974313$ & -0.2 \\
\hline$C$ & -10.091946 & $5-4.209504$ & -1.54 \\
\hline & -12.107793 & $3-3.835599$ & -0.09 \\
\hline $\mathrm{C}$ & -6.722782 & -2.983391 & -1.120087 \\
\hline & -6.274577 & -2.974177 & -2.442945 \\
\hline $\mathrm{C}$ & -4.389573 & -1.871798 & -1.814358 \\
\hline $\mathrm{C}$ & -4.825139 & -1.905171 & -0.485487 \\
\hline C & -14.040612 & $2-3.199951$ & -1.126764 \\
\hline $\mathrm{C}$ & -14.436468 & $3-2.561589$ & 0.05 \\
\hline $\mathrm{C}$ & $-12 \cdot 671283$ & $3-3.512049$ & 1.141271 \\
\hline $\mathrm{H}$ & -7.951026 & -3.177595 & 1.350638 \\
\hline & -10.706092 & $2-4.555811$ & -2.366999 \\
\hline $\mathrm{H}$ & -6.874681 & -3.415956 & -3.229307 \\
\hline & -4.218398 & -1.469584 & 0.300684 \\
\hline & -14.635268 & $3-3.144215$ & -2.030792 \\
\hline & -12.152828 & $3-3.713440$ & 2.070922 \\
\hline C & 3.103721 & -1.245346 & 2.210476 \\
\hline C & 1.128974 & -0.328309 & 1.578796 \\
\hline & 0.689359 & -0.233646 & 2.912353 \\
\hline & 1.540317 & -0.629919 & 3.929277 \\
\hline
\end{tabular}

\begin{tabular}{|c|c|c|c|}
\hline $\mathrm{C}$ & 2.777832 & -1.135596 & 3.567124 \\
\hline & -0.689359 & 0.233646 & 2.912353 \\
\hline & -1.128974 & 0.328309 & 1.578796 \\
\hline & -3.103721 & 1.245346 & 2.210476 \\
\hline & -2.777832 & 1.135596 & 3.567124 \\
\hline & -1.540317 & 0.629919 & 9277 \\
\hline & 1.245999 & -0.575425 & 4.972274 \\
\hline & 3.487979 & -1.483838 & 4.304981 \\
\hline & -3.487979 & 1.483838 & 4.3 \\
\hline & -1.245999 & 0.575425 & 4.9 \\
\hline & 3.103721 & 1.245346 & $-2 \cdot 2$ \\
\hline & 1.128974 & 0.328309 & -1.5 \\
\hline & 0.68 & 0.23 & -2.5 \\
\hline & 1.540317 & 0.629919 & -3.9 \\
\hline & 2.77 & 1.13 & -3.5 \\
\hline & -0.68 & -0.233646 & $-2 \cdot 9$ \\
\hline & -1.12 & -0.32 & -1.5 \\
\hline & -3.10 & -1.245346 & -2.2 \\
\hline & -2.77 & -1.13 & -3.5 \\
\hline & -1.54 & -0.62 & -3.9 \\
\hline & 1.24 & 0.57 & -4.9 \\
\hline & 3.48 & 1.483838 & $-4 \cdot 3$ \\
\hline & -3.48 & -1.48 & $-4 \cdot 3$ \\
\hline & -1.24 & -0.57 & -4.9 \\
\hline & -15.2 & -1.324245 & 16 \\
\hline & -15.2 & 1.324245 & -0 . \\
\hline & 15.24 & -1.324245 & -0.0 \\
\hline & 15.2 & 1.32 & \\
\hline C & -15.4 & $5-0.575512$ & 565 \\
\hline & -15.4 & 50.575512 & -1 \\
\hline $\mathrm{C}$ & 15.46 & -0.575512 & $-1 \cdot 2$ \\
\hline C & 15 . & 0.575512 & \\
\hline $\mathrm{H}$ & -15.577505 & 1.037920 & -2 \\
\hline & -15.5 & $5-1.037920$ & 009 \\
\hline $\mathrm{H}$ & 15.577 & -1.037920 & -2.179009 \\
\hline & 15.57 & 1.03 & 2.1 \\
\hline & 2.295143 & 0.844475 & -1.224917 \\
\hline $\mathrm{N}$ & 2.295 & -0.844475 & 1.2 \\
\hline & -2.295143 & 0.844475 & 1.22 \\
\hline $\mathrm{N}$ & -2.29 & -0.844475 & -1.22 \\
\hline & 5.124024 & 2.418393 & $-2.7 \varepsilon$ \\
\hline $\mathrm{N}$ & -5.12 & 2.41 & 2.7 \\
\hline & 5.124 & -2.418393 & $2.7 \xi$ \\
\hline $\mathrm{N}$ & -5.12 & -2.41 & $-2 \cdot 7$ \\
\hline & 5.985 & 2.450081 & -0.143068 \\
\hline $\mathrm{N}$ & -5.98 & 2.45 & 0.1 \\
\hline & 5.985 & -2.450081 & 0.14 \\
\hline $\mathrm{N}$ & -5.98 & -2.45 & -0.1 \\
\hline & 8.818666 & 3.951896 & -1.793514 \\
\hline $\mathrm{N}$ & -8.818 & 3.95 & 3514 \\
\hline & 8.818666 & -3.951896 & 1.793514 \\
\hline $\mathrm{N}$ & -8.818666 & -3.95 & -1.793514 \\
\hline $\mathrm{N}$ & 9.869914 & 3.657493 & 0.754403 \\
\hline $\mathrm{N}$ & -9.869914 & 3.657493 & -0.754403 \\
\hline $\mathrm{N}$ & 9.869914 & -3.657493 & -0.754403 \\
\hline . & -9.86 & -3.657 & 0.754403 \\
\hline & 12.858 & 3.786936 & -1.2 \\
\hline
\end{tabular}


$\begin{array}{llll}\mathrm{N} & -12.858458 & 3.786936 & 1.201604\end{array}$

$\mathrm{N} 12.858458-3.786936 \quad 1.201604$

$\mathrm{N}-12.858458-3.786936-1.201604$

$\begin{array}{llll}\mathrm{N} & 13.807055 & 2.836461 & 1.200880\end{array}$

$\mathrm{N}-13.807055 \quad 2.836461-1.200880$

$\mathrm{N} 13.807055-2.836461-1.200880$

$\mathrm{N}-13.807055-2.836461 \quad 1.200880$

N $15.402198 \quad-0.742573 \quad 1.149215$

$\mathrm{N}-15.402198-0.742573-1.149215$

$\begin{array}{llll}\mathrm{N}-15.402198 & 0.742573 & 1.149215\end{array}$

$\mathrm{N} 15.402198 \quad 0.742573-1.149215$

$\begin{array}{llll}\text { C } & 0.000000 & 0.000000 & 0.675607\end{array}$

C $0.000000 \quad 0.000000 \quad-0.675607$

$\Delta \mathrm{H}=-4744.127371$

\section{$5 \mathrm{CC}-8$}

$\begin{array}{lccc}\mathrm{C} & -8.036615 & 3.526626 & 0.715230 \\ \mathrm{C} & -8.544531 & 3.463571 & -0.585527 \\ \mathrm{C} & -10.542585 & 4.263588 & 0.146728 \\ \mathrm{C} & -9.991548 & 4.450050 & 1.418044 \\ \mathrm{C} & -11.997895 & 4.393844 & -0.081472 \\ \mathrm{C} & -6.724159 & 2.944781 & 1.076553 \\ \mathrm{C} & -6.302266 & 2.916578 & 2.407779 \\ \mathrm{C} & -4.412041 & 1.811393 & 1.803140 \\ \mathrm{C} & -4.832237 & 1.842808 & 0.469022 \\ \mathrm{C} & -14.064728 & 4.387712 & 0.872323 \\ \mathrm{C} & -14.596428 & 3.843223 & -0.303133 \\ \mathrm{C} & -12.579211 & 4.112315 & -1.322519 \\ \mathrm{H} & -7.934300 & 3.087978 & -1.398553 \\ \mathrm{H} & -10.582309 & 4.877521 & 2.219414 \\ \mathrm{H} & -6.912495 & 3.357282 & 3.186824 \\ \mathrm{H} & -4.218639 & 1.401759 & -0.308676 \\ \mathrm{H} & -14.684809 & 4.554364 & 1.745131 \\ \mathrm{H} & -11.979993 & 4.062139 & -2.223782 \\ \mathrm{C} & 8.036615 & -3.526626 & 0.715230 \\ \mathrm{C} & 8.544531 & -3.463571 & -0.585527 \\ \mathrm{C} & 10.542585 & -4.263588 & 0.146728 \\ \mathrm{C} & 9.991548 & -4.450050 & 1.418044 \\ \mathrm{C} & 11.997895 & -4.393844 & -0.081472 \\ \mathrm{C} & 6.724159 & -2.944781 & 1.076553 \\ \mathrm{C} & 6.302266 & -2.916578 & 2.407779 \\ \mathrm{C} & 4.412041 & -1.811393 & 1.803140 \\ \mathrm{C} & 4.832237 & -1.842808 & 0.469022 \\ \mathrm{C} & 14.064728 & -4.387712 & 0.872323 \\ \mathrm{C} & 14.596428 & -3.843223 & -0.303133 \\ \mathrm{C} & 12.579211 & -4.112315 & -1.322519 \\ \mathrm{H} & 7.934300 & -3.087978 & -1.398553 \\ \mathrm{H} & 10.582309 & -4.877521 & 2.219414 \\ \mathrm{H} & 6.912495 & -3.357282 & 3.186824 \\ \mathrm{H} & 4.218639 & -1.401759 & -0.308676 \\ \mathrm{H} & 14.684809 & -4.554364 & 1.745131 \\ \mathrm{H} & 11.979993 & -4.062139 & -2.223782 \\ \mathrm{C} & 8.036615 & 3.526626 & -0.715230 \\ \mathrm{C} & 8.544531 & 3.463571 & 0.585527 \\ \mathrm{C} & 10.542585 & 4.263588 & -0.146728\end{array}$

\begin{tabular}{|c|c|c|c|}
\hline 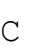 & 9.991548 & 4.450050 & -1.418044 \\
\hline & 11.997895 & 4.393844 & 0.081472 \\
\hline & 6.724159 & 2.944781 & -1.076553 \\
\hline & 6.302266 & 2.916578 & -2.407779 \\
\hline & 4.412041 & 1.811393 & -1.803140 \\
\hline & 4.832237 & 1.842808 & -0.469022 \\
\hline & 14.064728 & 4.387712 & -0.872323 \\
\hline & 14.596428 & 3.843223 & 0.303133 \\
\hline & 12.579211 & 4.112315 & 1.32 \\
\hline & 34300 & 3.087978 & 53 \\
\hline & 10.582309 & 4.877521 & $-2 \cdot 2$ \\
\hline & 6.912495 & 3.357282 & $-3.1 \varepsilon$ \\
\hline & 4.21 & 1.401 & 0.3 \\
\hline & 14.68 & 4.554 & $-1 \cdot 7$ \\
\hline & 11.97 & 4.06 & \\
\hline & -8.03 & -3.526626 & -0.7 \\
\hline & -8.5 & -3.46 & 0.5 \\
\hline & -10.5 & -4.2 & 728 \\
\hline & -9 . & -4.45 & $-1 \cdot 4$ \\
\hline & -11.9 & -4.3 & 472 \\
\hline & -6.72 & -2.94 & -1.0 \\
\hline & -6.30 & -2.916 & -2.4 \\
\hline & -4.41 & -1.81 & -1.8 \\
\hline & -4.83 & -1.842 & -0.4 \\
\hline & -14.0 & -4.387712 & -0 \\
\hline & -14.5 & -3.843223 & 33 \\
\hline C & -12.5 & -4.112315 & 519 \\
\hline & -7 & -3.087 & \\
\hline & -10.5 & -4.87 & -2 \\
\hline & -6 & -3.35 & -3 \\
\hline $\mathrm{H}$ & -4.21 & -1.401759 & 0.3 \\
\hline $\mathrm{H}$ & -14 & -4.554364 & -1 \\
\hline $\mathrm{H}$ & $-11 \cdot 9$ & -4.062139 & 782 \\
\hline $\mathrm{C}$ & 3.12 & -1.200 & \\
\hline C & 1.13 & -0.316303 & 1.5 \\
\hline C & 0.69 & -0.224 & 2.9 \\
\hline C & 1.55 & -0.603392 & 3.9 \\
\hline C & 2.79 & -1.08 & 3.5 \\
\hline $\mathrm{C}$ & -0.692 & 0.224012 & 2.9 \\
\hline $\mathrm{C}$ & -1.13 & 0.316 & 1.5 \\
\hline $\mathrm{C}$ & -3.12 & 1.200 & 2.2 \\
\hline $\mathrm{C}$ & -2.79 & 1.08 & 3.5 \\
\hline $\mathrm{C}$ & -1.55 & 0.603392 & 3.9 \\
\hline $\mathrm{H}$ & 1.26 & -0.54 & 4.9 \\
\hline $\mathrm{H}$ & $3.51^{\circ}$ & -1.423185 & 4.3 \\
\hline $\mathrm{H}$ & -3.51 & 1.42 & 4.3 \\
\hline $\mathrm{H}$ & -1.26 & 0.54 & 4.9 \\
\hline C & 3.12 & 1.20 & -2.2 \\
\hline $\mathrm{C}$ & 1.131421 & 0.316303 & -1.5 \\
\hline $\mathrm{C}$ & 0.69 & 0.22 & $-2 \cdot 9$ \\
\hline $\mathrm{C}$ & 1.553816 & 0.603392 & $-3 \cdot 9$ \\
\hline $\mathrm{C}$ & 2.799156 & 1.089940 & -3.5 \\
\hline $\mathrm{C}$ & -0.692960 & -0.224012 & $-2 \cdot 9$ \\
\hline $\mathrm{C}$ & -1.131421 & -0.316303 & -1.5 \\
\hline $\mathrm{C}$ & -3.1 & -1.200307 & $-2 \cdot 2$ \\
\hline $\mathrm{C}$ & -2.7 & -1.089940 & -3.5 \\
\hline C & -1.553 & -0.603392 & -3.9 \\
\hline
\end{tabular}




\begin{tabular}{|c|c|c|c|}
\hline $\mathrm{H}$ & 082 & 650 & -4 \\
\hline & 3.517361 & 1.423185 & -4.302653 \\
\hline & 61 & -1.423185 & -4.302 \\
\hline & 82 & -0 . & -4.97286 \\
\hline & -15.830970 & -3.032957 & 0.29046 \\
\hline & -17.142819 & -0.733287 & 0.0844 \\
\hline & 17.142819 & 0.733287 & 0.084456 \\
\hline & 15.830970 & 3.032957 & 0.290463 \\
\hline & 620 & 486 & 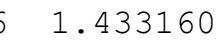 \\
\hline & -17.034038 & -1.600134 & -1.012905 \\
\hline & 17.034038 & 1.600134 & -1.012905 \\
\hline & 16.281620 & 2.357486 & 1.433160 \\
\hline & -17.418599 & -1.337040 & -1.9 \\
\hline & -16.056477 & -2.710561 & 223 \\
\hline & 17. & 1.3 & -1.99 \\
\hline & 477 & & \\
\hline & -15.830970 & 3.032957 & -0.290 \\
\hline & 970 & $-3 . c$ & -0.2904 \\
\hline & 2819 & & \\
\hline & & -0.7 & -0 . \\
\hline & -16 & 2. & $5-1$ \\
\hline & 16.28 & -2.35 & -1.43 \\
\hline & -17.034038 & 1.600134 & 1.012905 \\
\hline & 17.034038 & -1.600134 & 1.01 \\
\hline & 77 & 61 & -2 \\
\hline & 16. & -2.7 & -2.4 \\
\hline & 17.2 & -1.337040 & 1.9 \\
\hline & -17.418599 & 1.337040 & 1.99093 \\
\hline & 2.303238 & 0.817015 & -1.224862 \\
\hline & 2.303238 & -0.817015 & 1.22 \\
\hline & -2.3 & & \\
\hline & -2.3 & $-0 . \varepsilon$ & -1.2 . \\
\hline & 5.16 & 2.3 & -2.7 \\
\hline & -5.160775 & 2.351562 & 2.76 \\
\hline & 5.160775 & -2.351562 & 2.7 \\
\hline & -5.16 & -2.3 & -2.7 \\
\hline & 5.9 & 2.4 & -0.1 \\
\hline & -5.9 & 2.4 & 0 . \\
\hline & 031 & -2.400432 & 0. \\
\hline & -5.981031 & -2.400432 & -0.1 \\
\hline J & 8.75 & 7429 & $-1 \cdot 7$ \\
\hline & -8.7 & 4. & \\
\hline V & 8.7 & $-4 . c$ & 1 . \\
\hline & -8.7 & -4.0 & -1 \\
\hline & 846 & 3.8 & $0 . \varepsilon$ \\
\hline & -9.793846 & 3.810898 & $-0.8-2$. \\
\hline$y$ & 9.793846 & -3.810898 & -0.8 \\
\hline N & 3846 & -3.810898 & 0.8 \\
\hline N & 12.767881 & 4.622738 & -0.9 \\
\hline & -12.767881 & 4.622738 & 0.98464 \\
\hline & 12.767881 & -4.622738 & 0.984644 \\
\hline & -12.767881 & -4.62273 & -0.98464 \\
\hline & 13.863293 & 3.803655 & 1.41731 \\
\hline & 13.863293 & 3.803655 & $5-1.417319$ \\
\hline & & -3.8 & -1.417 \\
\hline & -13.863293 & & \\
\hline & 6.331091 & & -0.90441 \\
\hline
\end{tabular}

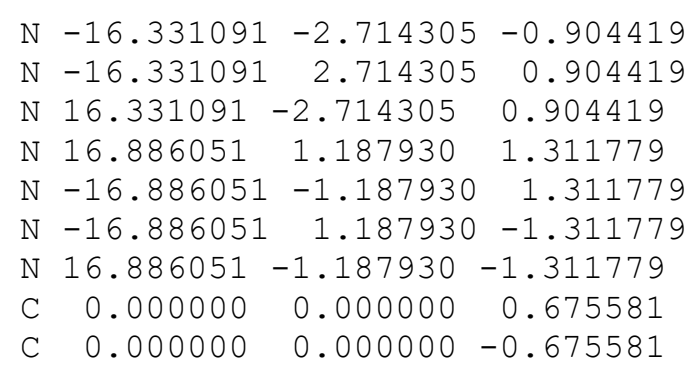

$\Delta \mathrm{H}=-5270.208090$

\section{$6 \mathrm{NN}-4$}

$\begin{array}{lrrr}\mathrm{C} & -7.855633 & 2.669199 & 0.759353 \\ \mathrm{C} & -9.523662 & 0.717822 & 0.191815 \\ \mathrm{C} & -9.489569 & 1.139644 & 1.524701 \\ \mathrm{C} & -6.442364 & 3.074937 & 0.965394 \\ \mathrm{C} & -3.890504 & 2.664114 & 1.563144 \\ \mathrm{C} & -4.214393 & 2.807434 & 0.210300 \\ \mathrm{H} & -10.002228 & 0.583451 & 2.298754 \\ \mathrm{H} & -3.456625 & 2.659388 & -0.548473 \\ \mathrm{C} & 7.855633 & -2.669199 & 0.759353 \\ \mathrm{C} & 9.523662 & -0.717822 & 0.191815 \\ \mathrm{C} & 9.489569 & -1.139644 & 1.524701 \\ \mathrm{C} & 6.442364 & -3.074937 & 0.965394 \\ \mathrm{C} & 3.890504 & -2.664114 & 1.563144 \\ \mathrm{C} & 4.214393 & -2.807434 & 0.210300 \\ \mathrm{H} & 10.002228 & -0.583451 & 2.298754 \\ \mathrm{H} & 3.456625 & -2.659388 & -0.548473 \\ \mathrm{C} & 7.855633 & 2.669199 & -0.759353 \\ \mathrm{C} & 9.523662 & 0.717822 & -0.191815 \\ \mathrm{C} & 9.489569 & 1.139644 & -1.524701 \\ \mathrm{C} & 6.442364 & 3.074937 & -0.965394 \\ \mathrm{C} & 3.890504 & 2.664114 & -1.563144 \\ \mathrm{C} & 4.214393 & 2.807434 & -0.210300 \\ \mathrm{H} & 10.002228 & 0.583451 & -2.298754 \\ \mathrm{H} & 3.456625 & 2.659388 & 0.548473 \\ \mathrm{C} & -1.855633 & -2.669199 & -0.759353 \\ \mathrm{C} & -9.523662 & -0.717822 & -0.191815 \\ \mathrm{C} & -9.489569 & -1.139644 & -1.524701 \\ \mathrm{C} & -6.442364 & -3.074937 & -0.965394 \\ \mathrm{C} & -3.890504 & -2.664114 & -1.563144 \\ \mathrm{C} & -4.214393 & -2.807434 & -0.210300 \\ \mathrm{H} & -1.10 .002228 & -0.583451 & -2.298754 \\ \mathrm{H} & -3.456625 & -2.659388 & 0.548473 \\ \mathrm{C} & 2.623031 & -2.028988 & 2.010473 \\ \mathrm{C} & 0.877770 & -0.721378 & 1.484528 \\ \mathrm{C} & 0.551519 & -0.462292 & 2.831014 \\ \mathrm{C} & 1.307121 & -1.103421 & 3.801189 \\ \mathrm{C} & 2.356871 & -1.900893 & 3.379581 \\ \mathrm{C} & -0.551519 & 0.462292 & 2.831014 \\ \mathrm{C} & -0.877770 & 0.721378 & 1.484528 \\ \mathrm{C} & -2.623031 & 2.028988 & 2.010473 \\ \mathrm{C} & -2.356871 & 1.900893 & 3.379581 \\ \mathrm{C} & -1.000 & 0.000000 & 0.676487\end{array}$




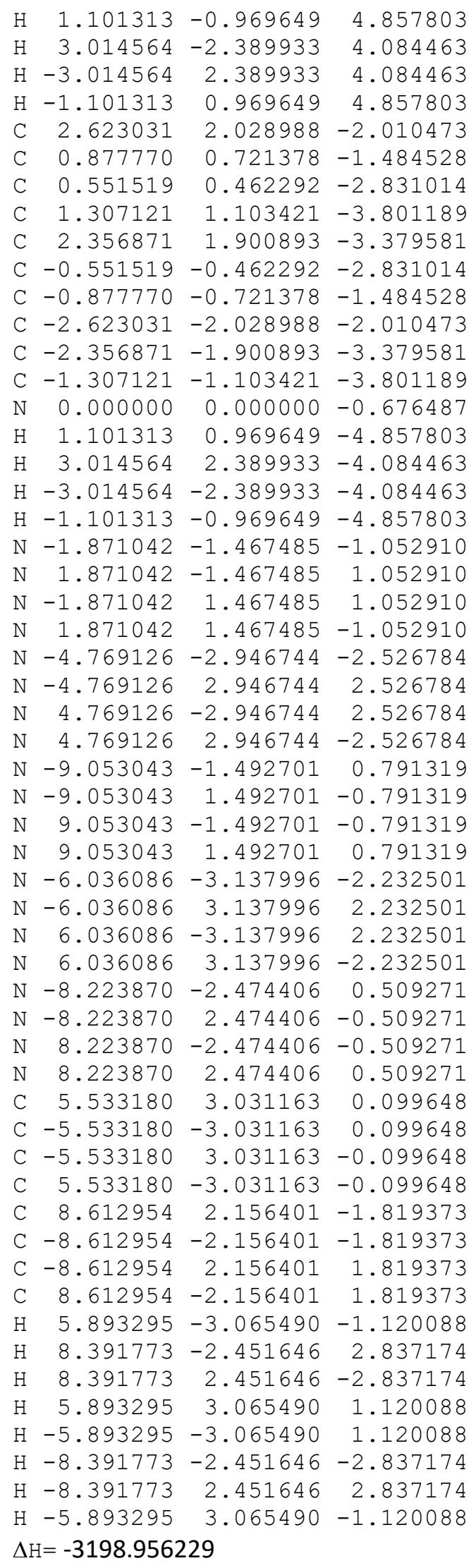

\section{$6 N N-6$}

\begin{tabular}{|c|c|c|c|}
\hline C & -7.532889 & 4.231063 & 0.791612 \\
\hline & -10.124593 & 3.826021 & 0.437966 \\
\hline & -9.710030 & 4.202783 & 1.720324 \\
\hline & -11.356502 & 3.031125 & 0.207024 \\
\hline & -6.079072 & 3.973230 & 0.949922 \\
\hline & -3.702843 & 2.932270 & 1.53 \\
\hline & -4.059260 & 3.007608 & 0.179774 \\
\hline & -12.653088 & 0.742984 & 0.001736 \\
\hline 0 & -11.7 & 2.652813 & -1.074585 \\
\hline & -10.41 & 4.224717 & 2.5 \\
\hline & -3.39 & 2.60 & -0.57 \\
\hline & $-11 \cdot 4$ & 3.216297 & $-1 \cdot 9$ \\
\hline & 7.53 & -4.231063 & 0.79 \\
\hline & 10.12 & -3.82 & 0.4 \\
\hline 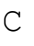 & 9.71 & -4.202 & 1.72 \\
\hline & 11.35 & -3.03 & 0.2 \\
\hline & & -3.97 & 0.9 \\
\hline 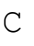 & 3.70 & -2.93 & 1.5 \\
\hline C & 4.05 & -3.00 & 0.1 \\
\hline 0 & 12.65 & -0.74 & 0.00 \\
\hline 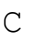 & 11.7 & -2.65 & -1.07 \\
\hline $\mathrm{H}$ & 10.41 & -4.224 & 2.53 \\
\hline $\mathrm{H}$ & 3.3 & -2.60 & -0.5 \\
\hline 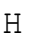 & 11.45 & -3.21 & -1.94 \\
\hline C & 7.53 & 4.23 & -0.7 \\
\hline C & 10.12 & 3.826 & -0.43 \\
\hline$C$ & 9.71 & 4.20 & -1.7 \\
\hline C & 11.356 & 3.031 & -0.20 \\
\hline C & 6.07 & 3.97 & -0.9 \\
\hline $\mathrm{C}$ & 3.702843 & 2.932270 & -1.53 \\
\hline $\mathrm{C}$ & 4.05 & 3.00 & -0.1 \\
\hline $\mathrm{C}$ & 12.653 & 0.742984 & -0.00 \\
\hline $\mathrm{C}$ & 11.77 & 2.65 & $1.0^{\circ}$ \\
\hline $\mathrm{H}$ & 10.416 & 4.2247 & $-2 \cdot 5$ \\
\hline $\mathrm{H}$ & 3.398 & 2.601 & 0.57 \\
\hline $\mathrm{H}$ & 11.453088 & 3.216297 & 1.9 \\
\hline $\mathrm{C}$ & -7.5 & -4.23 & -0.7 \\
\hline $\mathrm{C}$ & -10 & -3 & -0 \\
\hline$C$ & -9.7 & $-4 \cdot 2$ & -1.72 \\
\hline $\mathrm{C}$ & $-11 \cdot 3$ & -3.03 & -0 \\
\hline C & -6.0 & -3.973 & -0.94 \\
\hline $\mathrm{C}$ & -3.702 & -2.932270 & -1.53 \\
\hline $\mathrm{C}$ & -4.0 & -3.007 & -0.17 \\
\hline $\mathrm{C}$ & -12.65 & -0.742 & -0.0 \\
\hline $\mathrm{C}$ & -11.7 & -2.65 & 1. \\
\hline $\mathrm{H}$ & -10.41 & -4.224717 & -2 \\
\hline $\mathrm{H}$ & -3.39 & -2.601 & 0.57 \\
\hline $\mathrm{H}$ & -11 & -3.2 & \\
\hline $\mathrm{C}$ & 2.5 & -2.15 & 1.9 \\
\hline $\mathrm{C}$ & 0.85 & -0.7 & 1.4 \\
\hline C & 0.5 & -0.4 & 2.82 \\
\hline C & 1.2 & -1.1 & 3.7 \\
\hline $\mathrm{C}$ & 2.2 & -2.0 & 3.3 \\
\hline $\mathrm{C}$ & -0 . & & \\
\hline $\mathrm{C}$ & -0 & 0.7 & 1.4 \\
\hline
\end{tabular}




\begin{tabular}{|c|c|c|c|}
\hline & -2.524648 & 50646 & 07 \\
\hline & -2.280878 & 2.000454 & 3.361365 \\
\hline & -1.275386 & 1.150686 & 3.791660 \\
\hline & 0.000000 & 0.000000 & 0.675094 \\
\hline & 1.085637 & -1.008110 & 4.850112 \\
\hline & 2.910280 & -2.533958 & 4.059933 \\
\hline & -2.910280 & 2.533958 & 4.059933 \\
\hline & -1.085637 & 1.008110 & 4.850112 \\
\hline & 2.524648 & & \\
\hline & 0.851969 & 355 & -1 \\
\hline & 0.537254 & 0.480363 & -2.827549 \\
\hline & 1.275386 & 1.150686 & -3.79166 \\
\hline & 2.280878 & 2.000454 & -3.36136 \\
\hline & -0.537254 & -0.480363 & -2.8 \\
\hline & -0.851969 & -0.7 & -1.480020 \\
\hline & -2.524648 & $-2 \cdot 1$ & -1. \\
\hline & -2.280878 & -2.000454 & -3.361365 \\
\hline & -1.275386 & -1.150686 & -3.791660 \\
\hline & 0.000000 & 0.000000 & 5094 \\
\hline & 1.085637 & 1.008110 & -4.8 \\
\hline & 2.910280 & 2.5 & -4 . \\
\hline & -2.910280 & -2.533958 & -4.05993. \\
\hline & -1.085637 & -1.008110 & -4.850112 \\
\hline & -1.804152 & 1.540868 & 1.03950 \\
\hline & 1.804152 & -1.540868 & 1 . \\
\hline & 1.804152 & 1.5 & -1.0 \\
\hline & -1.804152 & -1.540868 & -1.0 \\
\hline & -4.452030 & 3.477056 & 2.48906 \\
\hline & 4.452030 & -3.477056 & 2.489067 \\
\hline & 4.452030 & 3.477056 & -2.489067 \\
\hline & -4.452030 & -3.477056 & -2.4 \\
\hline & -9.30 & 3.8 & -0.6 \\
\hline & 9.308660 & -3.881869 & -0.613805 \\
\hline & 9.308660 & 3.881869 & 0.613805 \\
\hline & -9.308660 & -3.881869 & 0.61380 \\
\hline & -11.845699 & 2.447946 & 1.301962 \\
\hline v & 11.84 & -2.447946 & 1.301962 \\
\hline 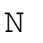 & 11.8 & 2.4 & -1.3 \\
\hline & -11.845699 & -2.447946 & -1.301962 \\
\hline & 5.629318 & 3.985835 & -2.202583 \\
\hline v & 8.019608 & 890 & 0.43914 \\
\hline $\mathrm{N}$ & 12.490364 & 1.304855 & -1.199697 \\
\hline N & 12.490364 & -1.304855 & 1.199697 \\
\hline $\mathrm{N}$ & 608 & -4.0 & -0.439140 \\
\hline 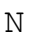 & 5.629318 & -3.985835 & 2.202583 \\
\hline - & -5.629318 & 3.985835 & 2.20258 \\
\hline iv & -8.019608 & 4.086890 & -0.439140 \\
\hline 2. & -12.490364 & 1.304855 & 1.199697 \\
\hline 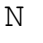 & -12.490364 & -1.304855 & $-1.19969^{\circ}$ \\
\hline $\mathrm{N}$ & -8.019608 & -4.086890 & 0.439140 \\
\hline - & -5.629318 & -3.985835 & -2.202583 \\
\hline C & 5.285181 & -3.547748 & 0.12169 \\
\hline C & -8.366230 & -4.411804 & -1.902831 \\
\hline 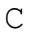 & -12.450512 & -1.464953 & 1.181016 \\
\hline 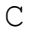 & -12.450512 & 1.464953 & $3-1.181016$ \\
\hline C & -8.366230 & 4.411804 & 1.902831 \\
\hline & 5.285181 & 50770 & \\
\hline
\end{tabular}

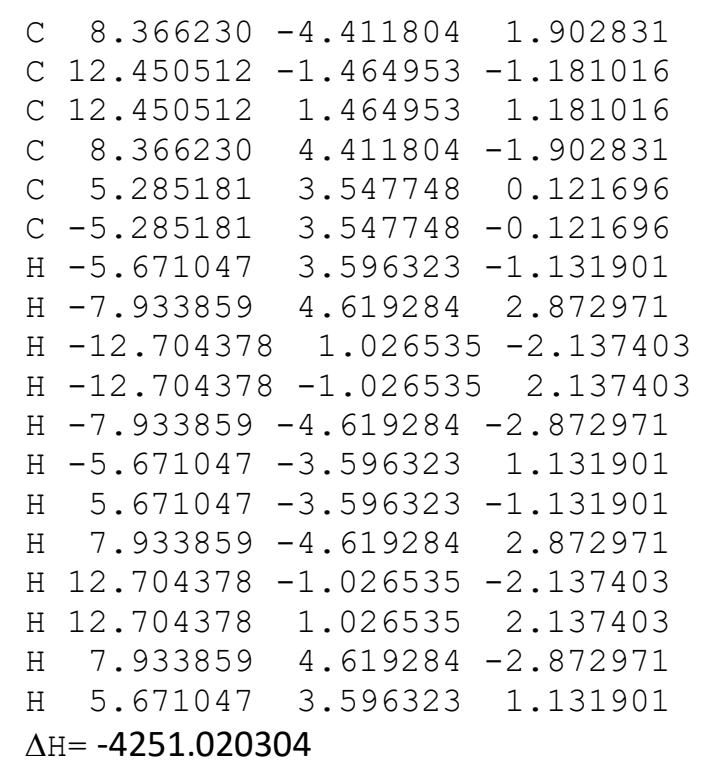

\section{$6 N N-8$}




\begin{tabular}{|c|c|c|c|}
\hline & 2112 & 953 & 569 \\
\hline & 11.097535 & 5.060026 & 2.136808 \\
\hline & -7.204559 & -4.907383 & -0.697745 \\
\hline & -9.786801 & -5.340468 & -0.277432 \\
\hline & -9.297378 & -5.594819 & -1.56387 \\
\hline & -11.239075 & -5.187524 & -0.0 \\
\hline & -5.838810 & -4.356576 & -0.88847 \\
\hline & -3.609634 & -3.045611 & -1.508905 \\
\hline & -3.956919 & -3.122466 & -0.155166 \\
\hline & -13.671598 & 54620 & 0.1555 \\
\hline & -11.733199 & -4.923585 & 1.271664 \\
\hline & -9.976020 & -5.890964 & -2.353112 \\
\hline & -3.342112 & -2.628953 & 0.586669 \\
\hline & -11 & & 36808 \\
\hline & 2.481863 & -2.200092 & 1.980451 \\
\hline & 0.84 & -0.76 & 1.47 \\
\hline & 0.531833 & -0.487231 & 2.826569 \\
\hline & 1.265226 & -1.168209 & 3.787229 \\
\hline & 2.251507 & -2.039095 & 3.352642 \\
\hline & -0.5 & 0 . & \\
\hline & -0.8 & 0. & $1.2+50$. \\
\hline & -2.48 & 2.200092 & 1.9 \\
\hline & -2.251507 & 0095 & 3.352642 \\
\hline & -1.2 & 1.1 & 3.787229 \\
\hline & 0.000000 & 00000 & 0.674615 \\
\hline & 78 & -1.0 & 4. \\
\hline & 2.8 & -2.5 & 4. \\
\hline & -2.868567 & 2.589230 & 4.0 \\
\hline & -1.083978 & 1.021740 & 4.846639 \\
\hline & 2.4 & 092 & -1.980451 \\
\hline & 0.8 & 25 & 8427 \\
\hline & 0.53 & 231 & -2.8 \\
\hline & 1.265226 & 1.1 & -3.7 \\
\hline & 2.251507 & 2.0 & 2642 \\
\hline & -0.531833 & -0.487231 & -2.826569 \\
\hline & -0.840652 & -0.760525 & -1.478427 \\
\hline & -2.2 & 092 & -1.980451 \\
\hline & -2.2 & -2.0 & -3.3 \\
\hline & -1.26 & -1.1 & -3.7 \\
\hline & 0.000000 & 0.000000 & -0.674615 \\
\hline & 1.083978 & 1740 & $-4.84663 s$ \\
\hline & 2.868567 & 2.589230 & -4.049340 \\
\hline & -2.868567 & -2.589230 & -4.049340 \\
\hline & -1.0 & -1.021740 & -4.8466 \\
\hline & -14 & -3.14952 & 006 \\
\hline & -15.850102 & -0.742076 & -0.01175 \\
\hline & 15.850102 & 0.742076 & -0.011757 \\
\hline & 14.761369 & 3.149528 & 0.067996 \\
\hline & -15.820316 & -1.464758 & -1.209713 \\
\hline & 15.820316 & 1.464758 & -1.20971 \\
\hline & -16.132404 & -1.000152 & -2.1358 \\
\hline & 16.132404 & 1.000152 & -2.135898 \\
\hline & -14.761369 & 3.149528 & -0.06799 \\
\hline & & -3.149528 & -0.067996 \\
\hline & -15.850102 & 0.742076 & 0.011757 \\
\hline & 15.850102 & -0.742076 & 0.011757 \\
\hline & -15.8203. & 1461 & 1200 \\
\hline
\end{tabular}

\begin{tabular}{|c|c|c|c|}
\hline & 316 & 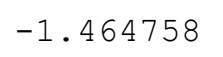 & \\
\hline & 32404 & -1.000152 & 2.135898 \\
\hline & 6.132404 & 1.000152 & \\
\hline & 1.773535 & 1.571354 & \\
\hline & 73535 & -1.571354 & 1.033684 \\
\hline & 1.773535 & 1.571354 & 3684 \\
\hline & -1.773535 & -1.571354 & -1.033684 \\
\hline & 4.307365 & 3.672776 & -2.454610 \\
\hline & -4.307365 & 3.672776 & \\
\hline & & -3.672776 & \\
\hline & $A$ & -3 & -2 . \\
\hline & 8.986382 & 5.033888 & 0.740596 \\
\hline & -8.986382 & 5.033888 & -0.740596 \\
\hline & 8.986382 & -5 & -0 \\
\hline & -8.9 & -5.0 & 0. \\
\hline & 12.0 & 5.1 & -1 \\
\hline & 12. & 5.12 & 1.0 \\
\hline & 12.011 & -5.126669 & 1.05 \\
\hline & -12.011988 & $8-5.126669$ & -1 \\
\hline & 15.5 & 1.312 & 1.1 \\
\hline & -15 & $3-1.3$ & 704 \\
\hline & -15 & 1.3 & $5-1$ \\
\hline & & -1.3 & $-1.1 \quad 1$ \\
\hline & & 4. & -2.14 \\
\hline & -5.410147 & 4.318191 & 2.14 \\
\hline & 5.4 & -4 & \\
\hline & -5 & -4 & -2 . \\
\hline & 7.7 & 4.8 & 0 . \\
\hline & -7.7 & 4.8 & -0.5 \\
\hline & 4 & -4.8 & -0.5 \\
\hline & -7 & -4 & 0. \\
\hline & 13. & 4.6 & -1 \\
\hline & -13 & 4.6 & 1. \\
\hline & 13.2 & -4.60 & 1. \\
\hline & -13. & $8-4.608787$ & -1.0 \\
\hline & 15.043576 & 2.511718 & 1.20 \\
\hline & -15 & $6-2.511718$ & 801 \\
\hline & -15 & $6 \quad 2.5=$ & $3-1$ \\
\hline & 15. & -2.5 & -1.2 \\
\hline & 5 & 3.7 & $0.1 \quad r \quad r$ \\
\hline & -5.10 & 3.798714 & -0.16631 \\
\hline $\mathrm{C}$ & 5.10 & -3.798714 & -0.166316 \\
\hline & -5.1 & -3.7 & $0.16 r$ \\
\hline & & 5. & -1 \\
\hline & -7.5 & 5.3 & \\
\hline 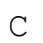 & & -5.3 & 1.7 t r t \\
\hline $\mathrm{C}$ & -7.962043 & -5.370806 & -1.780770 \\
\hline 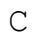 & 12.99 & 4.393200 & 1.35 \\
\hline & -12.995745 & 4.393200 & -1.35841 \\
\hline & & -4.39 & -1.358 \\
\hline & & & \\
\hline & 15.2 & 2.71498 & -1.167700 \\
\hline 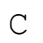 & -15.255527 & $7-2.714987$ & -1.167700 \\
\hline 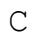 & -15.255527 & 2.714987 & 1.167700 \\
\hline & & -2.714987 & 1.1677 \\
\hline & & 3.87 & \\
\hline & $7 \quad 10063$ & 5.48 & 270 \\
\hline
\end{tabular}


$\begin{array}{lrrr}\text { H } & 13.432532 & 4.077984 & 2.297107 \\ \text { H } & 15.091705 & 3.310497 & -2.056443 \\ \text { H } & 15.091705 & -3.310497 & 2.056443 \\ \text { H } & 13.432532 & -4.077984 & -2.297107 \\ \text { H } & 7.499637 & -5.482400 & 2.752680 \\ \text { H } & 5.477886 & -3.877173 & -1.180425 \\ \text { H } & -5.477886 & 3.877173 & -1.180425 \\ \text { H } & -7.499637 & 5.482400 & 2.752680 \\ \text { H } & -13.432532 & 4.077984 & -2.297107 \\ \text { H } & -15.091705 & 3.310497 & 2.056443 \\ \text { H } & -15.091705 & -3.310497 & -2.056443 \\ \text { H } & -13.432532 & -4.077984 & 2.297107 \\ \text { H } & -7.499637 & -5.482400 & -2.752680 \\ \text { H } & -5.477886 & -3.877173 & 1.180425\end{array}$

$\Delta \mathrm{H}=-5303.071911$

\section{$6 \mathrm{CC}-4$}

$\begin{array}{lrcc}\mathrm{C} & -8.381515 & 2.324535 & 1.157515 \\ \mathrm{C} & -10.236957 & 0.675941 & 0.310423 \\ \mathrm{C} & -10.164126 & 0.864343 & 1.694339 \\ \mathrm{C} & -6.927222 & 2.516593 & 1.376463 \\ \mathrm{C} & -4.390104 & 1.896647 & 1.867758 \\ \mathrm{C} & -4.742136 & 2.141012 & 0.536592 \\ \mathrm{H} & -10.719448 & 0.233921 & 2.376605 \\ \mathrm{H} & -4.008654 & 2.007976 & -0.248799 \\ \mathrm{C} & 8.381515 & -2.324535 & 1.157515 \\ \mathrm{C} & 10.236957 & -0.675941 & 0.310423 \\ \mathrm{C} & 10.164126 & -0.864343 & 1.694339 \\ \mathrm{C} & 6.927222 & -2.516593 & 1.376463 \\ \mathrm{C} & 4.390104 & -1.896647 & 1.867758 \\ \mathrm{C} & 4.742136 & -2.141012 & 0.536592 \\ \mathrm{H} & 10.719448 & -0.233921 & 2.376605 \\ \mathrm{H} & 4.008654 & -2.007976 & -0.248799 \\ \mathrm{C} & 8.381515 & 2.324535 & -1.157515 \\ \mathrm{C} & 10.236957 & 0.675941 & -0.310423 \\ \mathrm{C} & 10.164126 & 0.864343 & -1.694339 \\ \mathrm{C} & 6.927222 & 2.516593 & -1.376463 \\ \mathrm{C} & 4.390104 & 1.896647 & -1.867758 \\ \mathrm{C} & 4.742136 & 2.141012 & -0.536592 \\ \mathrm{H} & 10.719448 & 0.233921 & -2.376605 \\ \mathrm{H} & 4.008654 & 2.007976 & 0.248799 \\ \mathrm{C} & -8.381515 & -2.324535 & -1.157515 \\ \mathrm{C} & -10.236957 & -0.675941 & -0.310423 \\ \mathrm{C} & -10.164126 & -0.864343 & -1.694339 \\ \mathrm{C} & -6.927222 & -2.516593 & -1.376463 \\ \mathrm{C} & -4.390104 & -1.896647 & -1.867758 \\ \mathrm{C} & -4.742136 & -2.141012 & -0.536592 \\ \mathrm{H} & -10.719448 & -0.233921 & -2.376605 \\ \mathrm{H} & -4.008654 & -2.007976 & 0.248799 \\ \mathrm{C} & 3.067842 & -1.330111 & 2.243451 \\ \mathrm{C} & 1.121304 & -0.364941 & 1.585402 \\ \mathrm{C} & 0.677813 & -0.260192 & 2.916319 \\ \mathrm{C} & 1.496682 & -0.694686 & 3.942910 \\ \mathrm{C} & 2.724666 & -1.228515 & 3.596080 \\ \mathrm{C} & -0.677813 & 0.260192 & 2.916319\end{array}$

$\begin{array}{lrrr}\text { C } & -1.121304 & 0.364941 & 1.585402 \\ \mathrm{C} & -3.067842 & 1.330111 & 2.243451 \\ \mathrm{C} & -2.724666 & 1.228515 & 3.596080 \\ \mathrm{C} & -1.496682 & 0.694686 & 3.942910 \\ \mathrm{H} & 1.185798 & -0.640447 & 4.981044 \\ \mathrm{H} & 3.423798 & -1.585211 & 4.340053 \\ \mathrm{H} & -3.423798 & 1.585211 & 4.340053 \\ \mathrm{H} & -1.185798 & 0.640447 & 4.981044 \\ \mathrm{C} & 3.067842 & 1.330111 & -2.243451 \\ \mathrm{C} & 1.121304 & 0.364941 & -1.585402 \\ \mathrm{C} & 0.677813 & 0.260192 & -2.916319 \\ \mathrm{C} & 1.496682 & 0.694686 & -3.942910 \\ \mathrm{C} & 2.724666 & 1.228515 & -3.596080 \\ \mathrm{C} & -0.677813 & -0.260192 & -2.916319 \\ \mathrm{C} & -1.121304 & -0.364941 & -1.585402 \\ \mathrm{C} & -3.067842 & -1.330111 & -2.243451 \\ \mathrm{C} & -2.724666 & -1.228515 & -3.596080 \\ \mathrm{C} & -1.496682 & -0.694686 & -3.942910 \\ \mathrm{H} & 1.185798 & 0.640447 & -4.981044 \\ \mathrm{H} & 3.423798 & 1.585211 & -4.340053 \\ \mathrm{H} & -3.423798 & -1.585211 & -4.340053 \\ \mathrm{H} & -1.185798 & -0.640447 & -4.981044 \\ \mathrm{~N} & -2.279451 & -0.914207 & -1.245139 \\ \mathrm{~N} & 2.279451 & -0.914207 & 1.245139 \\ \mathrm{~N} & -2.279451 & 0.914207 & 1.245139 \\ \mathrm{~N} & 2.279451 & 0.914207 & -1.245139 \\ \mathrm{~N} & -5.239689 & -2.111559 & -2.873123 \\ \mathrm{~N} & -5.239689 & 2.111559 & 2.873123 \\ \mathrm{~N} & 5.239689 & -2.111559 & 2.873123 \\ \mathrm{~N} & 5.239689 & 2.111559 & -2.873123 \\ \mathrm{~N} & -9.720148 & -1.579203 & 0.532748 \\ \mathrm{~N} & -9.720148 & 1.579203 & -0.532748 \\ \mathrm{~N} & 9.720148 & -1.579203 & -0.532748 \\ \mathrm{~N} & 9.720148 & 1.579203 & 0.532748 \\ \mathrm{~N} & -6.499821 & -2.399723 & -2.631384 \\ \mathrm{~N} & -6.499821 & 2.399723 & 2.631384 \\ \mathrm{~N} & 6.499821 & -2.399723 & 2.631384 \\ \mathrm{~N} & 6.499821 & 2.399723 & -2.631384 \\ \mathrm{~N} & -8.797774 & -2.414659 & 0.109656 \\ \mathrm{~N} & -8.797774 & 2.414659 & -0.109656 \\ \mathrm{H} & 6.42790 \\ \mathrm{H} & -6.427903 & -2.627232 & 0.718750 \\ \mathrm{H} & -8.916075 & -1.805362 & -3.180680 \\ \mathrm{H} & -8.916075 & 1.805362 & 3.180680 \\ \mathrm{~N} & 8.797774 & 2.414659 & 0.109656 \\ \mathrm{C} & 6.048377 & 2.478166 & -0.284285 \\ \mathrm{C} & -6.048377 & -2.478166 & -0.284285 \\ \mathrm{C} & -6.048377 & 2.478166 & 0.284285 \\ \mathrm{C} & 6.048377 & -2.478166 & 0.284285 \\ \mathrm{C} & 9.180385 & 1.719390 & -2.134145 \\ \mathrm{C} & -9.180385 & -1.719390 & -2.134145 \\ \mathrm{C} & -9.180385 & 1.719390 & 2.134145 \\ \mathrm{H} & 9.180385 & -1.719390 & 2.134145 \\ \mathrm{H} & 8.916075 & -1.805362 & 3.180680 \\ \end{array}$




$$
\begin{array}{rrrr}
\mathrm{H} & -6.427903 & 2.627232 & -0.718750 \\
\mathrm{C} & 0.000000 & 0.000000 & 0.677611 \\
\mathrm{C} & 0.000000 & 0.000000 & -0.677611
\end{array}
$$

\begin{tabular}{|c|c|c|c|}
\hline $\mathrm{C}$ & -8.188311 & 3.382101 & 1.201429 \\
\hline$C$ & -10.810853 & 3.414900 & 0.831163 \\
\hline $\mathrm{C}$ & -10.351677 & 3.604738 & 39345 \\
\hline C & -12.150076 & 2.849403 & 4624 \\
\hline C & -6.766841 & 2.972147 & 1.329402 \\
\hline $\mathrm{C}$ & -4.374720 & 1.914284 & 1.820389 \\
\hline $\mathrm{C}$ & -4.788745 & 1.976243 & 0.484742 \\
\hline C & -13.655306 & 0.738250 & 216 \\
\hline C & -12.615916 & 2.676652 & -0.7 \\
\hline $\mathrm{H}$ & -11.052194 & 3.666348 & 2.962061 \\
\hline $\mathrm{H}$ & -4.148691 & 1.580081 & -0.294337 \\
\hline $\mathrm{H}$ & -12.248932 & 3.301879 & -1.575804 \\
\hline $\mathrm{C}$ & 8.188311 & -3.382101 & 1.201429 \\
\hline C & 10.810853 & -3.414900 & 0.83 \\
\hline $\mathrm{C}$ & 10.351677 & -3.604738 & 2.13 \\
\hline C & 12.150076 & -2.849403 & 0.53 \\
\hline C & 6.766841 & -2.972147 & 1.329402 \\
\hline C & 4.374720 & -1.914284 & 1.82 \\
\hline $\mathrm{C}$ & 4.788745 & -1.976243 & 0.484742 \\
\hline C & 13.655306 & -0.738250 & 0.08 \\
\hline C & 12.615916 & -2.676652 & -0.7 \\
\hline $\mathrm{H}$ & 11.052194 & -3.666348 & 2.962061 \\
\hline $\mathrm{H}$ & 4.148691 & -1.580081 & -0.29 \\
\hline $\mathrm{H}$ & 12.248932 & -3.301879 & -1.575804 \\
\hline C & 8.16 & 3.382101 & $-1 \cdot 2$ \\
\hline $\mathrm{C}$ & 10.810853 & 3.414900 & -0.83 \\
\hline $\mathrm{C}$ & 10. & 3.604738 & -2.1 \\
\hline $\mathrm{C}$ & 12.150076 & 2.849403 & -0.534624 \\
\hline $\mathrm{C}$ & 6.766841 & 2.972147 & -1.32 \\
\hline C & 4.374720 & 1.914284 & -1.820389 \\
\hline $\mathrm{C}$ & 8745 & 1.976243 & -0.4 \\
\hline $\mathrm{C}$ & 13.655306 & 0.738250 & -0.086216 \\
\hline $\mathrm{C}$ & 12.615916 & 2.676652 & 0.772 \\
\hline $\mathrm{H}$ & 11.052194 & 3.666348 & -2.962061 \\
\hline $\mathrm{H}$ & 4.148691 & 1.580081 & 0.29 \\
\hline $\mathrm{H}$ & 12.248932 & 3.301879 & 1.575804 \\
\hline $\mathrm{C}$ & -8.18 & -3.382101 & -1.201429 \\
\hline $\mathrm{C}$ & -10.810853 & $3-3.414900$ & -0.8 \\
\hline C & -10.351677 & -3.604738 & $3-2.1$ \\
\hline $\mathrm{C}$ & -12.150076 & $5-2.849403$ & $3-0.53$ \\
\hline $\mathrm{C}$ & -6 . & -2.972147 & -1.329402 \\
\hline $\mathrm{C}$ & -4.374720 & -1.914284 & -1.82038 \\
\hline C & $-4.7 \varepsilon$ & -1.976243 & -0.484742 \\
\hline $\mathrm{C}$ & -13.655306 & -0.738250 & -0.086216 \\
\hline $\mathrm{C}$ & -12.615916 & -2.676652 & 2423 \\
\hline $\mathrm{H}$ & -11.052194 & -3.666348 & $3-2.962061$ \\
\hline $\mathrm{H}$ & $-4.14 \xi$ & -1.580081 & 0.2 \\
\hline $\mathrm{H}$ & -12.2 & -3.301879 & 804 \\
\hline 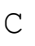 & 3.08 & -1.25 & 2.2 \\
\hline
\end{tabular}

$\Delta \mathrm{H}=-3165.63378$

\begin{tabular}{|c|c|c|c|}
\hline C & 1.125432 & -0.342810 & 1.580329 \\
\hline $\mathrm{C}$ & 0.684750 & -0.245562 & 2.912784 \\
\hline C & 1.523278 & -0.661283 & 3.932026 \\
\hline C & 2.753989 & -1.184844 & 3.574335 \\
\hline C & -0.684750 & 0.245562 & 2.912784 \\
\hline C & -1.125432 & 0.342810 & 1.580329 \\
\hline C & -3.084616 & 1.291259 & 2.218471 \\
\hline C & -2.753989 & 1.184844 & 3.5743 \\
\hline C & -1.523278 & 0.661283 & 3.932026 \\
\hline $\mathrm{H}$ & 1.224391 & -0.606940 & 4.973668 \\
\hline $\mathrm{H}$ & 3.456457 & -1.545341 & 4.313309 \\
\hline $\mathrm{H}$ & -3.456457 & 1.545341 & 4.313309 \\
\hline $\mathrm{H}$ & -1.224391 & 0.606940 & 4.973668 \\
\hline $\mathrm{C}$ & 3.084616 & 1.291259 & -2.218471 \\
\hline 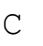 & 1.125432 & 0.342810 & -1.5 \\
\hline $\mathrm{C}$ & 0.684750 & 0.245562 & -2.912784 \\
\hline 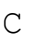 & 1.523278 & 0.661283 & $-3 \cdot 9$ \\
\hline $\mathrm{C}$ & 2.753989 & 1.184844 & -3.57 \\
\hline C & -0.684750 & -0.24 & $-2 \cdot 9$ \\
\hline $\mathrm{C}$ & -1.125432 & -0.342810 & -1.58 \\
\hline C & -3.08 & -1.29 & -2.2 \\
\hline $\mathrm{C}$ & -2.753989 & -1.184844 & -3.5 \\
\hline $\mathrm{C}$ & -1.523 & -0.66 & -3.9 \\
\hline $\mathrm{H}$ & 1.224391 & 0.606940 & -4.97 \\
\hline $\mathrm{H}$ & 3.45 & 1.54 & $-4 \cdot 3$ \\
\hline $\mathrm{H}$ & -3.4 & -1.54 & $-4 \cdot 3$ \\
\hline $\mathrm{H}$ & $-1 \cdot 22$ & -0.60 & $-4 \cdot 97$ \\
\hline $\mathrm{N}$ & -2 . & $0.8^{7}$ & 1.2 \\
\hline $\mathrm{N}$ & 2.2 & -0.87 & 1.2 \\
\hline $\mathrm{N}$ & 2 . & 0 . & $-1 \cdot 2$ \\
\hline $\mathrm{N}$ & -2.28 & -0.87 & -1.23 \\
\hline 10 & -5 & 2.4 & \\
\hline $\mathrm{N}$ & 5.11 & -2.40 & 2.8 \\
\hline $\mathrm{N}$ & 5 . & 2.4 & $-2 \cdot 8$ \\
\hline $\mathrm{N}$ & -5.112577 & -2.406871 & -2.81 \\
\hline $\mathrm{N}$ & -9 & 3.4194 & -0.2 \\
\hline $\mathrm{N}$ & 87454 & -3.419408 & -0.21 \\
\hline $\mathrm{N}$ & 9.987454 & 3.41 & 0.21 \\
\hline $\mathrm{N}$ & -9.987454 & -3.419408 & 0.216 \\
\hline $\mathrm{N}$ & -12 & 2.210744 & 1.56 \\
\hline $\mathrm{N}$ & 12.710483 & -2.210744 & 1.564 \\
\hline $\mathrm{N}$ & 12.710 & 2.210744 & -1.564157 \\
\hline $\mathrm{N}$ & -12.71 & -2.210744 & -1.56 \\
\hline $\mathrm{N}$ & 6.29 & 2.921492 & -2.572 \\
\hline $\mathrm{N}$ & 8.683 & 3.409332 & 0.033 \\
\hline $\mathrm{N}$ & 13.46 & 1.152890 & -1.339658 \\
\hline $\mathrm{N}$ & 13.460282 & -1.152890 & 1.339 \\
\hline $\mathrm{N}$ & 8.6 & -3.409332 & -0.033627 \\
\hline $\mathrm{N}$ & 6.296989 & -2.921492 & 2.572 \\
\hline $\mathrm{N}$ & -6.2 & 2.921492 & 2.572 \\
\hline $\mathrm{N}$ & -8.68 & 3.409332 & -0.033627 \\
\hline $\mathrm{N}$ & -13.46 & 1.152890 & 1.339658 \\
\hline $\mathrm{N}$ & -13.460282 & -1.152890 & -1.339658 \\
\hline $\mathrm{N}$ & -8.683 & -3.409332 & 0.033627 \\
\hline $\mathrm{N}$ & -6 & -2.921492 & -2.572 \\
\hline $\mathrm{C}$ & -6 & -2.527324 & -0.230 \\
\hline $\mathrm{C}$ & -8 & -3.585095 & -2.330 \\
\hline
\end{tabular}

\section{$6 C C-6$}




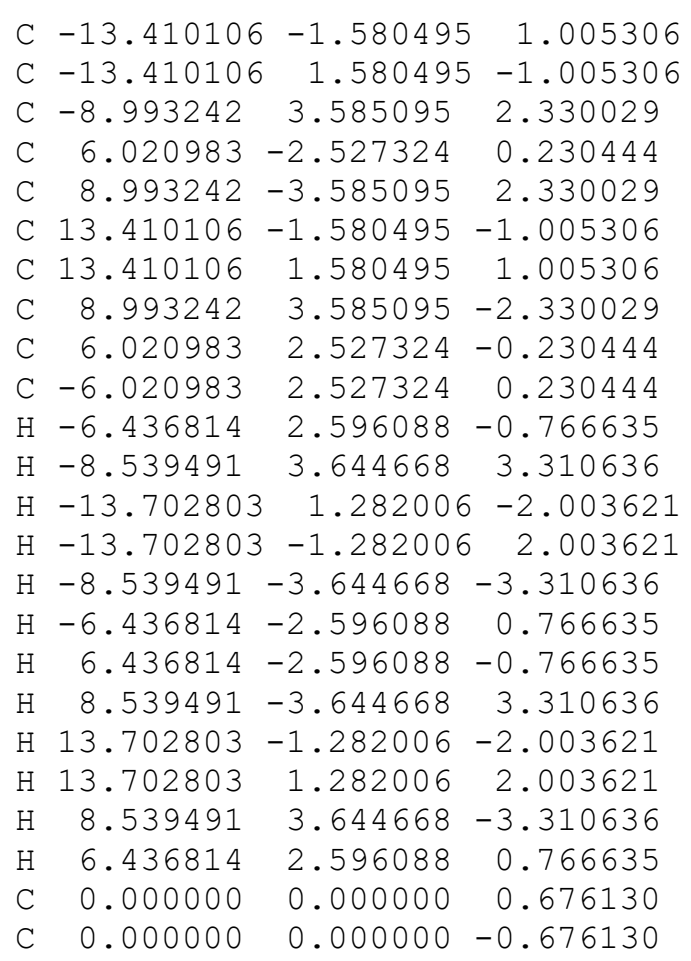

$\Delta \mathrm{H}=-4217.69592$

\section{$6 \mathrm{CC}-8$}

$\begin{array}{lrcr}\text { C } & -8.082419 & 3.589865 & 1.080571 \\ \text { C } & -10.591962 & 4.341920 & 0.658444 \\ \text { C } & -10.101308 & 4.454836 & 1.964498 \\ \text { C } & -12.042686 & 4.444201 & 0.361239 \\ \text { C } & -6.738075 & 2.980985 & 1.247155 \\ \text { C } & -4.408469 & 1.818879 & 1.794875 \\ \text { C } & -4.814693 & 1.847655 & 0.455138 \\ \text { C } & -14.614221 & 3.870358 & 0.097557 \\ \text { C } & -12.542744 & 4.343515 & -0.941677 \\ \text { H } & -10.753794 & 4.789135 & 2.760512 \\ \text { H } & -4.183952 & 1.405007 & -0.306409 \\ \text { H } & -11.871047 & 4.412023 & -1.787460 \\ \text { C } & 8.082419 & -3.589865 & 1.080571 \\ \text { C } & 10.591962 & -4.341920 & 0.658444 \\ \text { C } & 10.101308 & -4.454836 & 1.964498 \\ \text { C } & 12.042686 & -4.444201 & 0.361239 \\ \text { C } & 6.738075 & -2.980985 & 1.247155 \\ \text { C } & 4.408469 & -1.818879 & 1.794875 \\ \text { C } & 4.814693 & -1.847655 & 0.455138 \\ \text { C } & 14.614221 & -3.870358 & 0.097557 \\ \text { C } & 12.542744 & -4.343515 & -0.941677 \\ \text { H } & 10.753794 & -4.789135 & 2.760512 \\ \text { H } & 4.183952 & -1.405007 & -0.306409 \\ \text { H } & 11.871047 & -4.412023 & -1.787460 \\ \text { C } & 8.082419 & 3.589865 & -1.080571 \\ \text { C } & 10.591962 & 4.341920 & -0.658444 \\ \text { C } & 10.101308 & 4.454836 & -1.964498 \\ \text { C } & 12.042686 & 4.444201 & -0.361239\end{array}$

\begin{tabular}{|c|c|c|c|}
\hline U & 6.738075 & 2.980985 & -1.247155 \\
\hline C & 4.408469 & 1.818879 & -1.794875 \\
\hline 0 & 4.814693 & 1.847655 & -0.455138 \\
\hline & 14.614221 & 3.870358 & -0.097557 \\
\hline 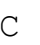 & 12.542744 & 4.343515 & 0.941677 \\
\hline & 10.753794 & 4.789135 & -2.760512 \\
\hline & 4.183952 & 1.405007 & 0.306409 \\
\hline & 11.871047 & 4.412023 & 1.787460 \\
\hline & -8.082419 & -3.58 & -1.080571 \\
\hline & -10.591962 & -4.341920 & -0.6 \\
\hline & -10.101308 & -4.454836 & -1 . \\
\hline & -12 . & -4.444201 & -0.3 \\
\hline & -6.7 & -2.98 & -1.24 \\
\hline & -4.408469 & -1.81 & -1.79 \\
\hline & -4.81 & $-1 \cdot 8$ & -0.4 \\
\hline & $-14 \cdot 6$ & -3.87 & -0 \\
\hline & -12.5 & -4.34 & \\
\hline & -10 & -4.7 & -2.7 \\
\hline & -4.1 & -1.4 & 0.30 \\
\hline & -11.8 & -4 & 160 \\
\hline $\mathrm{C}$ & 3.1 & -1.2 & 2.2 \\
\hline C & 1.1 & -0.3 & \\
\hline $\mathrm{C}$ & 0.6 & -0.22 & 2 . \\
\hline C & 1. & -0 . & \\
\hline C & 2.795 & -1. & 3. \\
\hline C & -0 . & 0. & \\
\hline $\mathrm{C}$ & -1 & 0.3 & 1.5 \\
\hline C & -3 & 1.2 & \\
\hline C & -2.795505 & 1.0 & 3.56 \\
\hline C & -1 . & 0 . & \\
\hline $\mathrm{H}$ & 1.258 & -0.5 & 4.97 \\
\hline $\mathrm{H}$ & 3 . & -1 & \\
\hline & -3.5116 & 1.4 & 4.30 \\
\hline $\mathrm{H}$ & -1 . & 0 & \\
\hline C & 3.118 & 1.2 & -2.21 \\
\hline $\mathrm{C}$ & 1.1 & 0.3 & -1.5 \\
\hline C & 0.6922 & 0.2 & -2.91 \\
\hline $\mathrm{C}$ & 1.55 & 0. & -3.9 \\
\hline C & 2.7955 & 1. & -3.56 \\
\hline 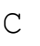 & -0.6922 & -0.22 & -2.91 \\
\hline 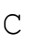 & $-1 \cdot 1$ & -0.31 & -1.58 \\
\hline $\mathrm{C}$ & -3.1 & $-1 \cdot 2$ & -2.21 \\
\hline 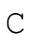 & -2.795 & -1.0 & -3.56 \\
\hline $\mathrm{C}$ & -1.55 & -0.6 & -3.93 \\
\hline 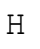 & 1.2584 & 0.5 & -4.973492 \\
\hline $\mathrm{H}$ & 3.511 & 1.4 & -4.3 \\
\hline $\mathrm{H}$ & -3.511 & -1.43 & -4.305746 \\
\hline $\mathrm{H}$ & -1.25 & -0.55 & -4.973492 \\
\hline $\mathrm{C}$ & -15.844401 & -3.038614 & -0.111844 \\
\hline $\mathrm{C}$ & -17.1 & -0 & -0.048999 \\
\hline 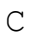 & 17.139537 & 0.740661 & -0.048999 \\
\hline $\mathrm{C}$ & 15.84 & 3.038614 & -0.111844 \\
\hline$G$ & -17.08 & -1.402696 & -1.280670 \\
\hline $\mathrm{C}$ & 17.088244 & 1.402696 & -1.280670 \\
\hline 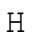 & -17.45 & -0.921703 & -2.178430 \\
\hline $\mathrm{H}$ & 17.4 & 0.9 & -2.178430 \\
\hline & -15 & 614 & \\
\hline
\end{tabular}




\begin{tabular}{|c|c|c|c|}
\hline C & 15.844401 & -3.038614 & 344 \\
\hline C & -17.139537 & 0.740661 & 0.048999 \\
\hline & 17.139537 & -0.740661 & 0.048999 \\
\hline & -17.088244 & 1.402696 & 1.280670 \\
\hline & 17.088244 & -1.402696 & 1.280670 \\
\hline & 17.453953 & -0.921703 & 2.178430 \\
\hline & -17.453953 & 0.921703 & 2.178430 \\
\hline & 2.302977 & 0.820476 & -1.227551 \\
\hline & 2.302977 & -0.820476 & 1.227551 \\
\hline & -2.302977 & 0.820476 & 1.227551 \\
\hline N & -2.302977 & -0.820476 & -1.227551 \\
\hline & 5.141886 & 2.351060 & -2.77 \\
\hline $\mathbb{N}$ & -5.14 & 2.351060 & 2.7 \\
\hline $\mathrm{N}$ & 5.141 & -2.351060 & 2.77 \\
\hline $\mathrm{N}$ & -5.14 & -2.351060 & -2.77 \\
\hline N & 9.822766 & 3.974258 & 0.36 \\
\hline $\mathrm{N}$ & -9.822766 & 3.974258 & -0.36 \\
\hline N & 9.822766 & -3.974258 & -0.36 \\
\hline N & -9.82 & -3.974258 & 0.36 \\
\hline $\mathrm{N}$ & 12.84 & 4.466086 & -1.42 \\
\hline N & -12.8 & 4.466086 & 1 . \\
\hline $\mathrm{N}$ & 12.84 & -4.466086 & 1.42 \\
\hline $\mathrm{N}$ & -12.8 & -4.466086 & $5-1$. \\
\hline $\mathrm{N}$ & 16.827 & 1.356847 & 1.05 \\
\hline N & -16.8 & -1.356847 & 1. \\
\hline N & -16.827329 & 1.356847 & $7-1.0$ \\
\hline $\mathrm{N}$ & 16.82 & -1.356847 & -1.09 \\
\hline $\mathrm{N}$ & 6 . & 2.922 & -2.4 \\
\hline N & -6.29 & 2.922 & 2.4 \\
\hline N & 6. & -2.922678 & 2.4 \\
\hline $\mathrm{N}$ & -6.29 & -2.922678 & -2.4 \\
\hline $\mathrm{N}$ & 8 . & 3.60 & 0.1 \\
\hline $\mathrm{N}$ & -8.57 & 3.604380 & -0.15 \\
\hline $\mathrm{N}$ & 8 . & -3.60 & -0.1 \\
\hline $\mathrm{N}$ & -8.575 & -3.604380 & 0.15 \\
\hline $\mathrm{N}$ & 14. & 4.1734 & $-1 \cdot 2$ \\
\hline $\mathrm{N}$ & -14.118900 & 4.173443 & 1.29 \\
\hline $\mathrm{N}$ & 14.11 & -4.173443 & 1.2 \\
\hline $\mathrm{N}$ & -14.118900 & -4.173443 & $3-1.29$ \\
\hline $\mathrm{N}$ & 16.184062 & 2.504351 & 1.0 \\
\hline $\mathrm{N}$ & -16.184062 & -2.504351 & 1.06 \\
\hline $\mathrm{N}$ & -16.1 & 2.50 & -1.06 \\
\hline $\mathrm{N}$ & 16.18 & -2.504351 & -1.061055 \\
\hline $\mathrm{C}$ & 6.01 & 2.447913 & -0.17 \\
\hline $\mathrm{C}$ & -6.017210 & 2.447913 & 0.171840 \\
\hline $\mathrm{C}$ & 6.01 & -2.447913 & 0.1 \\
\hline C & -6.017210 & -2.447913 & -0.171840 \\
\hline $\mathrm{C}$ & 8.805321 & 4.065336 & -2.182208 \\
\hline $\mathrm{C}$ & -8.805321 & 4.065336 & 2.182208 \\
\hline $\mathrm{C}$ & 8.805 & -4.065336 & 2.182208 \\
\hline C & -8.805321 & -4.065336 & -2.182208 \\
\hline $\mathrm{C}$ & 13.877054 & 4.053967 & 1.078909 \\
\hline $\mathrm{C}$ & -13.877054 & 4.053967 & $7-1.078909$ \\
\hline C & 13.877054 & -4.053967 & -1.078909 \\
\hline $\mathrm{C}$ & -13.877054 & -4.053967 & $7 \quad 1.078909$ \\
\hline $\mathrm{C}$ & 16.412551 & 2.597 & -1.312819 \\
\hline$C$ & -16.412551 & -2.597534 & $4-1.312819$ \\
\hline
\end{tabular}

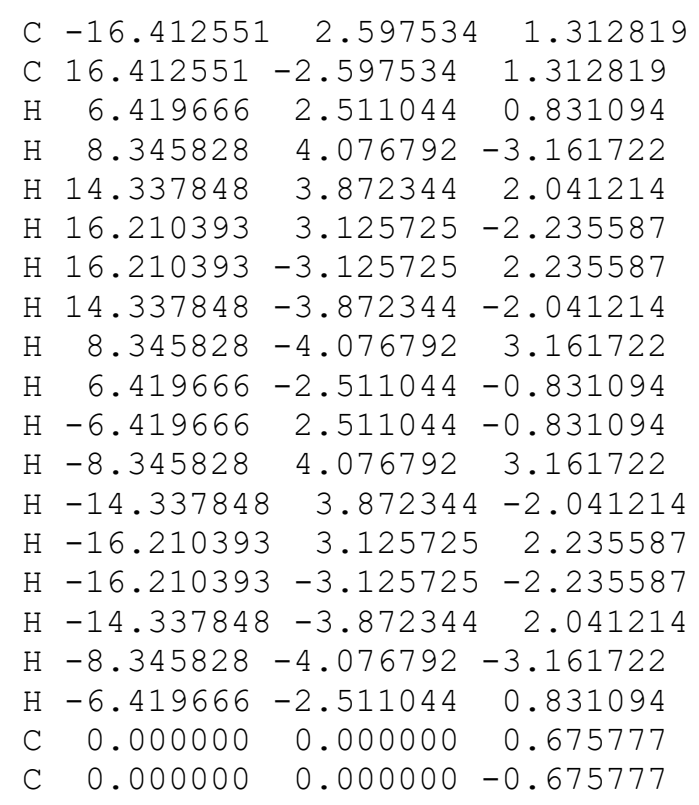

$\Delta \mathrm{H}=-5269.748315$

\section{NN-4}

$\begin{array}{rrrr}\text { C } & -8.071950 & 2.580476 & 1.161920 \\ \text { C } & -9.698340 & 0.688501 & 0.268385 \\ \text { C } & -6.682154 & 2.953924 & 1.497993 \\ \text { C } & -4.087175 & 2.556558 & 1.977636 \\ \text { C } & 8.071950 & -2.580476 & 1.161920 \\ \text { C } & 9.698340 & -0.688501 & 0.268385 \\ \text { C } & 6.682154 & -2.953924 & 1.497993 \\ \text { C } & 4.087175 & -2.556558 & 1.977636 \\ \text { C } & 8.071950 & 2.580476 & -1.161920 \\ \text { C } & 9.698340 & 0.688501 & -0.268385 \\ \text { C } & 6.682154 & 2.953924 & -1.497993 \\ \text { C } & 4.087175 & 2.556558 & -1.977636 \\ \text { C } & -8.071950 & -2.580476 & -1.161920 \\ \text { C } & -9.698340 & -0.688501 & -0.268385 \\ \text { C } & -6.682154 & -2.953924 & -1.497993 \\ \text { C } & -4.087175 & -2.556558 & -1.977636 \\ \text { C } & 2.764956 & -1.927532 & 2.219578 \\ \text { C } & 0.907199 & -0.686518 & 1.482889 \\ \text { C } & 0.576968 & -0.434379 & 2.824028 \\ \text { C } & 1.372412 & -1.015928 & 3.805466 \\ \text { C } & -0.576968 & 0.434379 & 2.824028 \\ \text { C } & -0.907199 & 0.686518 & 1.482889 \\ \text { C } & -2.764956 & 1.927532 & 2.219578 \\ \text { C } & -1.372412 & 1.015928 & 3.805466 \\ \text { N } & 0.000000 & 0.000000 & 0.680208 \\ \text { H } & 1.151734 & -0.883734 & 4.861238 \\ \text { H } & -1.151734 & 0.883734 & 4.861238 \\ \text { C } & 2.764956 & 1.927532 & -2.219578 \\ \text { C } & 0.907199 & 0.686518 & -1.482889 \\ \text { C } & 0.576968 & 0.434379 & -2.824028 \\ \text { C } & 1.372412 & 1.015928 & -3.805466 \\ \text { C } & -0.576968 & -0.434379 & -2.824028\end{array}$




\begin{tabular}{|c|c|c|c|}
\hline C & -0.907199 & -0.686518 & -1 \\
\hline & -2.764956 & -1.927532 & -2 \\
\hline & -1.372412 & -1.015928 & 0 \\
\hline & 0.00 & 000 & -0.6 \\
\hline & 1.15 & & \\
\hline & -1.15 & -0.883734 & \\
\hline & -2.01 & 1.452192 & \\
\hline & 2.01 & -1.452 & -1.1 \\
\hline & & -1.4 & \\
\hline & 2.011 & & $-1 \cdot 1$ \\
\hline & -5.01 & -2 & \\
\hline & -5.01 & 2.71 & \\
\hline & & -2.7 & \\
\hline & 5.0107 & 2.71 & -3.0 \\
\hline & -5.72 & -3.06 & \\
\hline & -5.72 & 3.06 & .7 \\
\hline & 5.72 & -3 & \\
\hline & 5.72 & 3.06 & -0 \\
\hline & -8.83 & -1 & \\
\hline & -8 & 1.8 & \\
\hline & & $-1 \cdot 8$ & \\
\hline & 8.83 & 1 . & -2 \\
\hline & -9.3 & 1.7 & \\
\hline & -9.3 & $-1 \cdot 7$ & 0 \\
\hline & 9.7 & $-1 \cdot 7$ & -0 \\
\hline & 9.3 & 1.7 & \\
\hline & -2.4 & -1.7 & -3 \\
\hline & 2. & -1 & \\
\hline & -2.4 & 1. & \\
\hline & 2.4 & 1 . & \\
\hline & -4.4 & $-2 \cdot \varepsilon$ & -0.7 \\
\hline & -4 & & \\
\hline & 4. & -2 . & 0 \\
\hline & & & \\
\hline & -6 & -2 . & -2.7 \\
\hline & -6 . & & \\
\hline & 6.3 & $-2 \cdot 8$ & 2.1 \\
\hline & 6.3 & 2 . & -2.7 \\
\hline & -8.4 & $-2 \cdot 6$ & 0 \\
\hline & -8 & 2 . & \\
\hline & 8.4 & $-2 \cdot 6$ & -0.1 \\
\hline & 8.4 & 2 . & \\
\hline & -9.58 & -0 . & -1.5 \\
\hline & -9.5 & & 7 \\
\hline & 9.5 & -0 . & 1.5 \\
\hline & 9 & & -1 \\
\hline & 2.32 & 1.6 & -0.1 \\
\hline & $-2 \cdot 3$ & -1 & -0.1 \\
\hline & $-2 \cdot 3$ & 1.6 & 0.1 \\
\hline & 2.3 & -1.6 & 0.1 \\
\hline & 4.6 & -2.6 & 4.0 \\
\hline & 6.0 & -3.2 & -0.5 \\
\hline & 8.73 & $-1 \cdot 9$ & 3.1 \\
\hline & 9.60 & $-1 \cdot \varepsilon$ & -1 \\
\hline & & & \\
\hline & 8.7 & & -3 \\
\hline & 6.0 & 3. & 0. \\
\hline
\end{tabular}

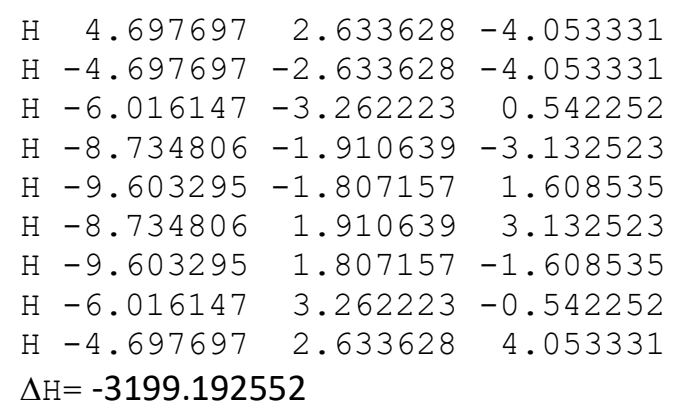

$7 \mathrm{NN}-5$ 


\begin{tabular}{|c|c|c|c|}
\hline $\mathrm{C}$ & -4.807871 & 3.101783 & 3.028082 \\
\hline C & 4.807871 & 3.101783 & -3.028082 \\
\hline 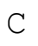 & -4.807871 & -3.101783 & -3.028082 \\
\hline C & 5.570626 & -3.403568 & 0.498939 \\
\hline$C$ & -5.570626 & 3.403568 & 0.498939 \\
\hline $\mathrm{C}$ & 5.570626 & 3.403568 & -0.498939 \\
\hline C & -5.570626 & -3.403568 & -0.498939 \\
\hline & 8.837399 & -3.194730 & 2.322380 \\
\hline $\mathrm{C}$ & -8.837399 & 3.194730 & 2.322380 \\
\hline & 8.837399 & 3.194730 & -2.322380 \\
\hline$C$ & -8.837399 & -3.194730 & -2.322380 \\
\hline & 9.553031 & 3.119466 & 0.24 \\
\hline C & 9.553031 & -3.119466 & -0.2 \\
\hline$C$ & -9.553031 & 3.119466 & -0.24 \\
\hline C & -9.553031 & -3.119466 & 0.2 \\
\hline C & 11.348159 & -0.249907 & 1.310 \\
\hline$C$ & -11.348159 & -0.249907 & $7-1.31$ \\
\hline $\mathrm{C}$ & 11.348159 & 0.249907 & -1.310451 \\
\hline C & -11.348159 & 0.249907 & 1.310451 \\
\hline $\mathrm{C}$ & 1.959687 & -1.517566 & 1.139858 \\
\hline C & -1.959687 & 1.517566 & 1.1 \\
\hline $\mathrm{C}$ & 1.959687 & 1.517566 & -1.139858 \\
\hline C & -1.959687 & -1.517566 & -1.139858 \\
\hline $\mathrm{N}$ & 2.374030 & -1.830339 & 3.501059 \\
\hline $\mathrm{N}$ & -2.374030 & 1.830339 & 3.5 \\
\hline $\mathrm{N}$ & 2.374030 & 1.830339 & -3.501059 \\
\hline $\mathrm{N}$ & -2.374030 & -1.830339 & -3.5 \\
\hline $\mathrm{N}$ & 4.331210 & -2.982666 & 0.706702 \\
\hline $\mathrm{N}$ & -4.331210 & 2.982666 & 0.706702 \\
\hline $\mathrm{N}$ & 4.331210 & 2.982666 & -0.706702 \\
\hline $\mathrm{N}$ & -4.331210 & -2.982666 & -0.706702 \\
\hline $\mathrm{N}$ & 6.066400 & -3.455083 & 2.819879 \\
\hline $\mathrm{N}$ & -6.066400 & 3.455083 & 2.819879 \\
\hline $\mathrm{N}$ & 6.066400 & 3.455083 & -2.819879 \\
\hline $\mathrm{N}$ & -6.066400 & -3.455083 & -2.819879 \\
\hline $\mathrm{N}$ & 8.356744 & -3.607478 & 0.041802 \\
\hline $\mathrm{N}$ & -8.356744 & 3.607478 & 0.041802 \\
\hline $\mathrm{N}$ & 8.356744 & 3.607478 & -0.0 \\
\hline $\mathrm{N}$ & -8.356744 & -3.607478 & -0.041802 \\
\hline $\mathrm{N}$ & 80410 & -2.597990 & 2.02 \\
\hline $\mathrm{N}$ & -9.980410 & 2.597990 & 2.027062 \\
\hline $\mathrm{N}$ & 9.980410 & 2.597990 & -2.027062 \\
\hline $\mathrm{N}$ & -9.980410 & -2.597990 & -2.027062 \\
\hline $\mathrm{N}$ & 11.272905 & -1.011188 & -0.923207 \\
\hline $\mathrm{N}$ & 11.272905 & 1.011188 & 0.923207 \\
\hline $\mathrm{N}$ & -11.272905 & 1.011188 & $3-0.923207$ \\
\hline $\mathrm{N}$ & -11.272905 & -1.011188 & 0.923207 \\
\hline $\mathrm{H}$ & 2.259214 & -1.716309 & 8796 \\
\hline $\mathrm{H}$ & -2.259214 & 1.716309 & 0.118796 \\
\hline $\mathrm{H}$ & -2.259214 & -1.716309 & -0.1 \\
\hline $\mathrm{H}$ & 2.259214 & 1.716309 & -0.118796 \\
\hline $\mathrm{H}$ & 4.468536 & -3.020663 & 4.053119 \\
\hline $\mathrm{H}$ & 5.880700 & -3.570134 & -0.526072 \\
\hline $\mathrm{H}$ & 8.579167 & -3.307981 & 3.368415 \\
\hline $\mathrm{H}$ & 9.884714 & -3.161804 & -1.273061 \\
\hline 1 & 11.461435 & -0.45225 & 2.368583 \\
\hline 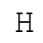 & 11.461435 & 0.452252 & -2.368583 \\
\hline
\end{tabular}

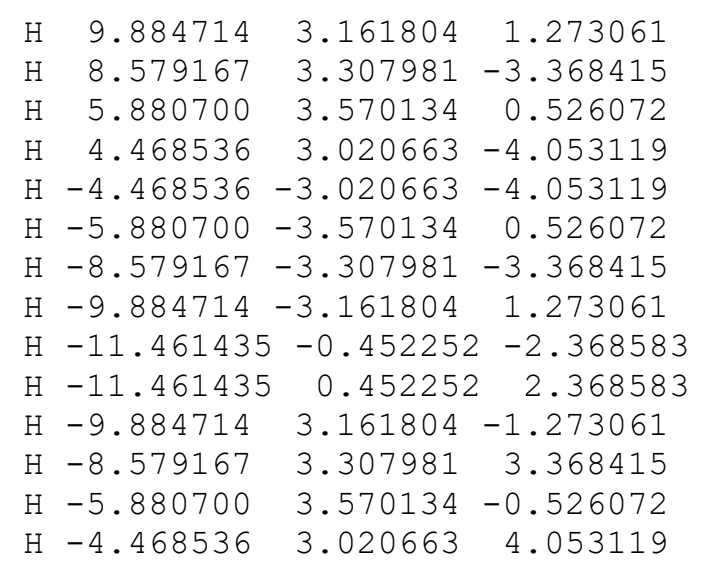

$\Delta \mathrm{H}=-3725.278415$

\section{$7 \mathrm{NN}-6$}

$\begin{array}{lccc}\mathrm{C} & -7.744201 & 4.166853 & 1.348585 \\ \mathrm{C} & -9.482385 & 4.076545 & -0.116073 \\ \mathrm{C} & -10.344573 & 3.745723 & 0.937115 \\ \mathrm{C} & -8.653044 & 4.113185 & 2.412839 \\ \mathrm{C} & -11.580208 & 2.974460 & 0.685524 \\ \mathrm{C} & -6.300560 & 3.927105 & 1.565651 \\ \mathrm{C} & -4.660830 & 3.338375 & 3.023621 \\ \mathrm{C} & -3.866206 & 2.895847 & 1.960048 \\ \mathrm{C} & -5.444662 & 3.632077 & 0.499878 \\ \mathrm{C} & -12.183695 & 2.229675 & 1.709502 \\ \mathrm{C} & -12.888116 & 0.723236 & 0.150989 \\ \mathrm{C} & -12.625670 & 1.662750 & -0.857580 \\ \mathrm{H} & -9.838993 & 4.120734 & -1.138332 \\ \mathrm{H} & -8.316275 & 4.195426 & 3.439207 \\ \mathrm{H} & -4.312383 & 3.246894 & 4.044709 \\ \mathrm{H} & -5.763004 & 3.787245 & -0.524282 \\ \mathrm{H} & -12.083491 & 2.509947 & 2.751123 \\ \mathrm{H} & -12.886362 & 1.475136 & -1.892233 \\ \mathrm{C} & 7.744201 & -4.166853 & 1.348585 \\ \mathrm{C} & 9.482385 & -4.076545 & -0.116073 \\ \mathrm{C} & 10.344573 & -3.745723 & 0.937115 \\ \mathrm{C} & 8.653044 & -4.113185 & 2.412839 \\ \mathrm{C} & 11.580208 & -2.974460 & 0.685524 \\ \mathrm{C} & 6.300560 & -3.927105 & 1.565651 \\ \mathrm{C} & 4.660830 & -3.338375 & 3.023621 \\ \mathrm{C} & 3.866206 & -2.895847 & 1.960048 \\ \mathrm{C} & 5.444662 & -3.632077 & 0.499878 \\ \mathrm{C} & 12.183695 & -2.229675 & 1.709502 \\ \mathrm{C} & 12.888116 & -0.723236 & 0.150989 \\ \mathrm{C} & 12.625670 & -1.662750 & -0.857580 \\ \mathrm{H} & 9.838993 & -4.120734 & -1.138332 \\ \mathrm{H} & 8.316275 & -4.195426 & 3.439207 \\ \mathrm{H} & 4.312383 & -3.246894 & 4.044709 \\ \mathrm{H} & 5.763004 & -3.787245 & -0.524282 \\ \mathrm{H} & 12.083491 & -2.509947 & 2.751123 \\ \mathrm{H} & 12.886362 & -1.475136 & -1.892233 \\ \mathrm{C} & 7.744201 & 4.166853 & -1.348585 \\ \mathrm{C} & 9.482385 & 4.076545 & 0.116073\end{array}$




\begin{tabular}{|c|c|c|c|}
\hline $\mathrm{C}$ & 10.344573 & 3.745723 & -0.937115 \\
\hline $\mathrm{C}$ & 8.653044 & 4.113185 & -2.412839 \\
\hline C & 11.580208 & 2.974460 & -0.685524 \\
\hline C & 6.300560 & 3.927105 & -1.565651 \\
\hline C & 4.660830 & 3.338375 & -3.023621 \\
\hline $\mathrm{C}$ & 3.866206 & 2.895847 & -1.960048 \\
\hline $\mathrm{C}$ & 5.444662 & 3.632077 & -0.499878 \\
\hline $\mathrm{C}$ & 12.183695 & 2.229675 & -1.709502 \\
\hline C & 12.888116 & 0.723236 & -0.150989 \\
\hline C & 12.625670 & 1.662750 & 0.857580 \\
\hline $\mathrm{H}$ & 9.838993 & 4.120734 & 1.138332 \\
\hline $\mathrm{H}$ & 8.316275 & 4.195426 & -3.439207 \\
\hline $\mathrm{H}$ & 4.312383 & 3.246894 & -4.044709 \\
\hline $\mathrm{H}$ & 5.763004 & 3.787245 & 0.524282 \\
\hline $\mathrm{H}$ & 12.083491 & 2.509947 & -2.751123 \\
\hline $\mathrm{H}$ & 12.886362 & 1.475136 & 1.89 \\
\hline C & -7.744201 & -4.166853 & $-1 \cdot 34$ \\
\hline $\mathrm{C}$ & -9.482385 & -4.076545 & 0.116073 \\
\hline C & $-10 \cdot 34$ & -3.745723 & $3-0$. \\
\hline $\mathrm{C}$ & -8.65 & -4.11 & -2.41 \\
\hline $\mathrm{C}$ & -11.5 & -2.97 & -0 . \\
\hline $\mathrm{C}$ & -6.300560 & -3.927105 & -1.565651 \\
\hline $\mathrm{C}$ & -4.66 & -3.338375 & -3.02 \\
\hline $\mathrm{C}$ & -3.866206 & -2.895847 & -1.960048 \\
\hline $\mathrm{C}$ & -5.44 & -3.632077 & -0.45 \\
\hline $\mathrm{C}$ & -12.1 & -2.229675 & -1 . \\
\hline $\mathrm{C}$ & $-12 \cdot 8$ & -0.723236 & $5-0$. \\
\hline $\mathrm{C}$ & $-12 \cdot 6$ & -1.662750 & 80 \\
\hline $\mathrm{H}$ & -9.83 & -4.120734 & 1.1 \\
\hline $\mathrm{H}$ & -8.31 & -4.195426 & -3.4 \\
\hline $\mathrm{H}$ & -4.31 & -3.246894 & -4.044709 \\
\hline $\mathrm{H}$ & -5.76 & -3.78 & 0.52 \\
\hline $\mathrm{H}$ & -12.0 & -2.509947 & -2 \\
\hline $\mathrm{H}$ & $-12 \cdot \varepsilon$ & -1.475136 & \\
\hline C & 2.635440 & -2.102377 & 2.20 \\
\hline C & 1.924572 & -1.559717 & 1.1 \\
\hline C & 0.872501 & -0.728827 & 1.479335 \\
\hline $\mathrm{C}$ & 0.554405 & -0.463922 & 2.82 \\
\hline C & 1.315203 & -1.097039 & 3.79 \\
\hline C & -0.554405 & 0.463922 & 2.82 \\
\hline $\mathrm{C}$ & -0.872501 & 0.728827 & 1.479335 \\
\hline $\mathrm{C}$ & -1.924572 & 1.559717 & 1.1 \\
\hline C & -2.635440 & 2.102377 & 2.204159 \\
\hline $\mathrm{C}$ & -1.315203 & 1.097039 & 3.79 \\
\hline $\mathrm{N}$ & 0.000000 & 0.000000 & 0.678809 \\
\hline $\mathrm{H}$ & 2.216887 & -1.765543 & 0.114142 \\
\hline $\mathrm{H}$ & 1.108985 & -0.952913 & 4.854988 \\
\hline $\mathrm{H}$ & -2.216887 & 1.765543 & 0.114142 \\
\hline $\mathrm{H}$ & -1.108985 & 0.952913 & 4.85498 \\
\hline $\mathrm{C}$ & 2.635440 & 2.102377 & -2.204159 \\
\hline C & 1.924572 & 1.559717 & -1.135851 \\
\hline $\mathrm{C}$ & 0.872501 & 0.728827 & -1.47 \\
\hline C & 0.554405 & 0.463922 & -2.820780 \\
\hline C & 1.315203 & 1.097039 & -3.797880 \\
\hline C & -0.554405 & -0.463922 & -2.82078 \\
\hline C & -0.872501 & -0.728827 & -1.47 \\
\hline $\mathrm{C}$ & -1.924572 & -1.559717 & -1.13 \\
\hline
\end{tabular}

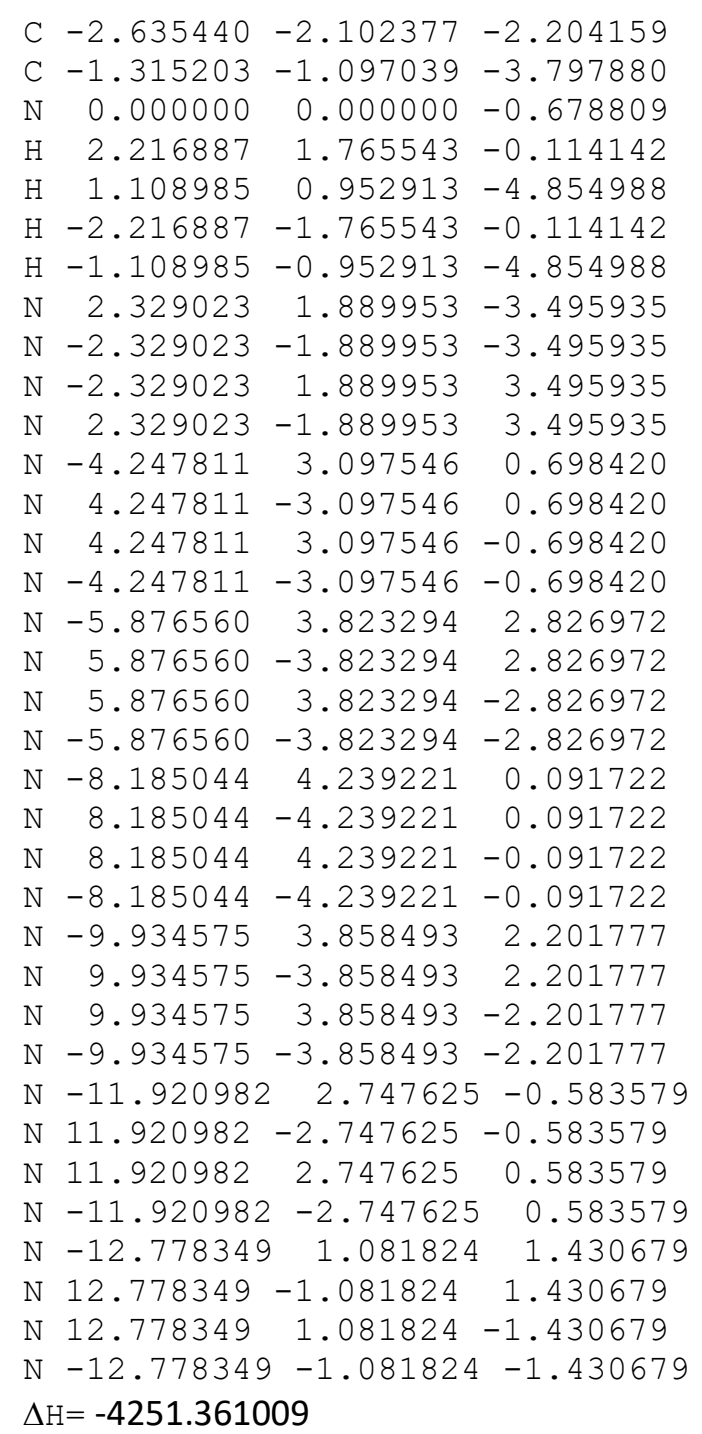

\section{NN- 7}




\begin{tabular}{|c|c|c|c|}
\hline C & 13.685714 & 2.617703 & -0.419521 \\
\hline & 565665 & -4.587131 & -1.342096 \\
\hline & -10.210146 & -4.6 & -0 . \\
\hline & 617534 & -4.252920 & 4340 \\
\hline & 6.159202 & -4.178846 & -1.553617 \\
\hline & 301701 & -2.981685 & -1.950446 \\
\hline & 13.685714 & -2.617703 & -0.4 \\
\hline & 2.600325 & -2.145768 & 2.199014 \\
\hline & 0.863129 & -0.739245 & 1.47 \\
\hline & 0.548746 & -0.471070 & 2.82 \\
\hline & 1.301701 & -1.116260 & 3.79 \\
\hline & -0.548746 & 0.471070 & 2.82 \\
\hline & -0.863129 & 0.739245 & $1.4^{\circ}$ \\
\hline & 2.600325 & 2.145768 & 2.1 \\
\hline & -1.301701 & 1.116260 & 3.7 \\
\hline & 0 . & 0.000000 & $0.6^{\circ}$ \\
\hline & 1.100555 & -0.968648 & 4.8 \\
\hline & 00555 & 0.968648 & 4.85 \\
\hline & 2.600325 & 2.145768 & -2.1 \\
\hline & 0.863129 & 0.73 & $-1 \cdot 4$ \\
\hline & 0.548746 & 0.471070 & -2.82 \\
\hline & 1.30 & 1.11 & -3.7 \\
\hline$C$ & -0.54 & -0.47 & -2.82 \\
\hline & -0 . & -0.73 & $-1 \cdot 4$ \\
\hline$c$ & -2.60 & -2.145768 & -2.19 \\
\hline & -1 & -1.11 & -3.7 \\
\hline & 0.000000 & 0.000000 & -0.67 \\
\hline $\mathrm{H}$ & 1.10 & 0.96 & -4.8 \\
\hline 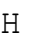 & -1.10 & -0.968 & $-4 \cdot 8$ \\
\hline & $-14 \cdot 3$ & -1.31 & -0.2 \\
\hline o & -14.347612 & 1.313929 & 474 \\
\hline C & 14.347612 & -1.313929 & 0.2 \\
\hline o & 14.347612 & 1.313929 & -0.2 \\
\hline $\mathbf{N}$ & 2.30 & $-1.92^{7}$ & 3.4 \\
\hline $\mathrm{N}$ & -2.300311 & 1.927792 & 3.4 \\
\hline $\mathrm{N}$ & 2.300311 & 1.92 & -3.4 \\
\hline $\mathrm{N}$ & -2.300311 & -1.927792 & -3.4 \\
\hline $\mathrm{C}$ & 1.900513 & -1.586680 & 1.1 \\
\hline $\mathrm{C}$ & 1.900513 & 1.586680 & $-1 \cdot 1$ \\
\hline $\mathrm{C}$ & -1.900513 & 1.586680 & 1.1 \\
\hline $\mathrm{C}$ & -1.900513 & -1.586680 & $-1 \cdot 1$ \\
\hline $\mathrm{C}$ & 4.560835 & -3.48 & 3.0 \\
\hline $\mathrm{C}$ & 4.560835 & 3.486592 & -3.01 \\
\hline $\mathrm{C}$ & -4.56 & 3.48 & 3.0 \\
\hline $\mathrm{C}$ & -4.560 & -3.486592 & -3.0 \\
\hline $\mathrm{C}$ & 5 . & -3.788711 & 0.4 \\
\hline C & 5.34 & 3.788711 & -0.488107 \\
\hline C & $-5.342 \varepsilon$ & 3.788711 & 0.4 \\
\hline $\mathrm{C}$ & -5 & -3.788711 & -0.4 \\
\hline C & 8.445209 & -4.735372 & 2.420672 \\
\hline C & 8.445209 & 4.735372 & -2.4 \\
\hline $\mathrm{C}$ & -8.445209 & 4.735372 & 2.420672 \\
\hline C & -8.445209 & -4.735372 & -2.420672 \\
\hline C & 9.324166 & -4.735813 & -0.090679 \\
\hline $\mathrm{C}$ & 24166 & & 679 \\
\hline C & -9.324166 & 4.735813 & -0.090679 \\
\hline & -9 . & -4.73581 & 0.0 \\
\hline
\end{tabular}

\begin{tabular}{|c|c|c|c|}
\hline & 12.397891 & -3.809393 & 1.869878 \\
\hline & 97891 & 3.809393 & -1.869878 \\
\hline & -12.397891 & 3.809393 & 1.869878 \\
\hline & 12.397891 & -3.809393 & $B-1.869878$ \\
\hline & 3105122 & -3.312919 & -0.649817 \\
\hline & 3.105122 & 3.312919 & 0.649817 \\
\hline & 13.105122 & 3.312919 & $9-0$ \\
\hline & 13.105122 & -3.312919 & 0.649817 \\
\hline & 4.532122 & 0.416587 & $-1 \cdot 2$ \\
\hline & 32122 & -0.416587 & 1.26 \\
\hline & 14.532122 & -0.416587 & $7-1$ \\
\hline & 14.53 & 0.416587 & 1.265941 \\
\hline & & -3.178219 & 0.6 \\
\hline & 4.182 & 3.178219 & 0.68 \\
\hline & & 3.17 & -0.6 \\
\hline & 14 & -3.17 & -0.6 \\
\hline & & -4.06 & \\
\hline & 5.737278 & 4.060068 & 2.81 \\
\hline & 5 . & 4.06 & -2.8 \\
\hline & 37278 & -4.060068 & -2.8 \\
\hline & & -4.6624 & \\
\hline & 13830 & 4.662420 & 0.08 \\
\hline & 8 & 4.66 & -0.0 \\
\hline & 30 & -4.662420 & -0.08 \\
\hline & 9. & -4.73 & 2.2 \\
\hline & 9. & 4.735529 & 2.24 \\
\hline & 44 & 4.73 & -2.24 \\
\hline & -9 & -4.735529 & -2.24 \\
\hline & 12 & -4.083167 & -0.45 \\
\hline & 0 & 4.083167 & $7-0.4$ \\
\hline & 12 . & 4.083167 & 0.45 \\
\hline & -12 & -4.083167 & .74 \\
\hline & 13 & -2.957756 & 1.67 \\
\hline & -1 & & 466 \\
\hline & 13.391 & 2.957 & -1.67 \\
\hline & -13 & -2 & $5-1$. \\
\hline & 1 & $-0 . \varepsilon$ & -1 \\
\hline & -14 & $-0 . \varepsilon$ & 897 \\
\hline & -14.472 & 0.887446 & $5-1$. \\
\hline N & 14 & 0.887446 & 1. \\
\hline & 58 & 1.797486 & -0.11 \\
\hline $\mathrm{H}$ & -2 . & -1.797 & -0.1 \\
\hline $\mathrm{H}$ & -2 & 1.797486 & 0.1 \\
\hline $\mathrm{H}$ & & -1.797 & 0.1 \\
\hline & 4. & -3.381900 & 4.03 \\
\hline $\mathrm{H}$ & 5 . & -3.9 & -0.5 \\
\hline & 8.0726 & -4.785 & 3.4 \\
\hline $\mathrm{H}$ & 9. & -4.78 & -1.1 \\
\hline & 12.150975 & -4.074914 & 2.8 \\
\hline $\mathrm{H}$ & 13. & -3.16 & -1.6 \\
\hline & 14 . & -0.759451 & 2.2 \\
\hline $\mathrm{H}$ & 14 . & 0.759451 & -2.2 \\
\hline & 13. & 3.163787 & 1.6 \\
\hline $\mathrm{H}$ & 12 . & 4.074914 & -2.890756 \\
\hline & & 4.78 & 1.1 \\
\hline & 8 . & 4.785969 & -3.4 \\
\hline & & 3.941304 & \\
\hline
\end{tabular}




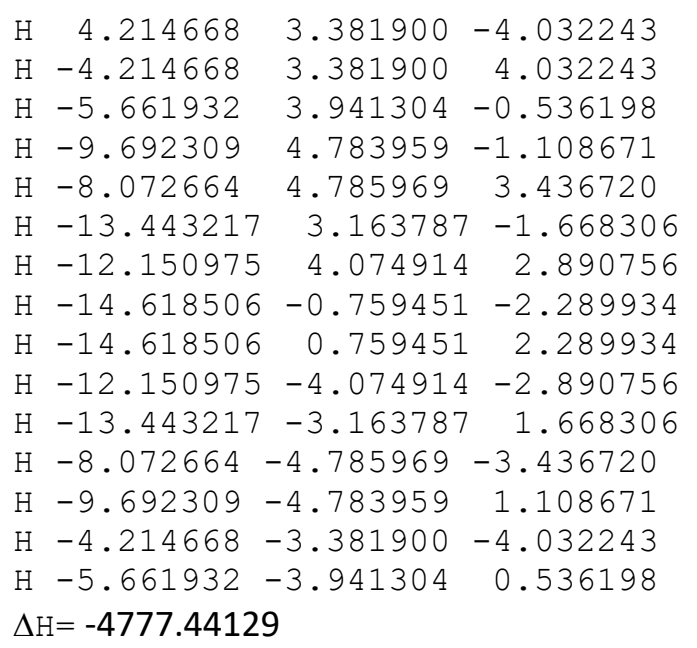

\section{$7 \mathrm{NN}-8$}

$\begin{array}{lccc}\text { C } & -7.414915 & 4.882441 & 1.335236 \\ \text { C } & -10.029479 & 5.311278 & 1.001954 \\ \text { C } & -11.492649 & 5.182702 & 0.826552 \\ \text { C } & -6.047743 & 4.356947 & 1.546887 \\ \text { C } & -3.753204 & 3.043380 & 1.946068 \\ \text { C } & -13.935379 & 4.155142 & 0.531246 \\ \text { C } & 7.414915 & -4.882441 & 1.335236 \\ \text { C } & 10.029479 & -5.311278 & 1.001954 \\ \text { C } & 11.492649 & -5.182702 & 0.826552 \\ \text { C } & 6.047743 & -4.356947 & 1.546887 \\ \text { C } & 3.753204 & -3.043380 & 1.946068 \\ \text { C } & 13.935379 & -4.155142 & 0.531246 \\ \text { C } & 7.414915 & 4.882441 & -1.335236 \\ \text { C } & 10.029479 & 5.311278 & -1.001954 \\ \text { C } & 11.492649 & 5.182702 & -0.826552 \\ \text { C } & 6.047743 & 4.356947 & -1.546887 \\ \text { C } & 3.753204 & 3.043380 & -1.946068 \\ \text { C } & 13.935379 & 4.155142 & -0.531246 \\ \text { C } & -7.414915 & -4.882441 & -1.335236 \\ \text { C } & -10.1029479 & -5.311278 & -1.001954 \\ \text { C } & -11.1492649 & -5.182702 & -0.826552 \\ \text { C } & -6.047743 & -4.356947 & -1.546887 \\ \text { C } & -1.3 .753204 & -3.043380 & -1.946068 \\ \text { C } & -13.935379 & -4.155142 & -0.531246 \\ \text { C } & 2.574017 & -2.177105 & 2.196779 \\ \text { C } & 0.856248 & -0.746879 & 1.478091 \\ \text { C } & 0.544503 & -0.476144 & 2.819873 \\ \text { C } & 1.291371 & -1.129509 & 3.794644 \\ \text { C } & -0.544503 & 0.476144 & 2.819873 \\ \text { C } & -0.856248 & 0.746879 & 1.478091 \\ \text { C } & -2.574017 & 2.177105 & 2.196779 \\ \text { C } & -1.1991371 & 1.129509 & 3.794644 \\ \text { N } & 0.000000 & 0.000000 & 0.678309 \\ \text { H } & 1.093659 & -0.978894 & 4.852450 \\ \text { H } & -1.1 .093659 & 0.978894 & 4.852450 \\ \text { C } & 2.574017 & 2.177105 & -2.196779 \\ \text { C } & 0.856248 & 0.746879 & -1.478091\end{array}$

\begin{tabular}{|c|c|c|c|}
\hline $\mathrm{C}$ & 0.544503 & 0.476144 & -2.819873 \\
\hline C & 1.291371 & 1.129509 & -3.794644 \\
\hline C & -0.544503 & -0.476144 & -2.819873 \\
\hline & -0.856248 & -0.746879 & -1.4 \\
\hline 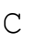 & -2.574017 & -2.177105 & -2.196779 \\
\hline & -1.291371 & -1.129509 & -3.794644 \\
\hline & 0.000000 & 0.000000 & -0.678309 \\
\hline & 1.093659 & 0.978894 & -4.85 \\
\hline & -1.093659 & -0.978894 & -4.852450 \\
\hline & -15.009268 & -3.151714 & -0.37164 \\
\hline & -16.086808 & -0.734677 & -0 \\
\hline & 16.086808 & 0.734677 & -0.07 \\
\hline & 15.00 & 3.15 & -0.3 \\
\hline & -15.009268 & 3.151714 & 644 \\
\hline & 15.0 & -3.151714 & $0.3^{\circ}$ \\
\hline & -16.0 & 0.734677 & 894 \\
\hline C & 16. & -0.734677 & $0.0^{\circ}$ \\
\hline $\mathrm{N}$ & 5.630264 & 4.223090 & -2.80 \\
\hline & -5 & 4.22 & 2 \\
\hline $\mathrm{N}$ & 5.6 & -4.22 & 2.80 \\
\hline & -5 & -4.22 & -2 \\
\hline $\mathrm{N}$ & 7.865521 & 4.96 & -0.08 \\
\hline & -7.8 & 4.9 & \\
\hline $\mathrm{N}$ & 7.8 & -4.96 & 0 . \\
\hline & -7 & -4.96 & -0 . \\
\hline & 13. & 4.43 & $-1 \cdot 7$ \\
\hline $\mathrm{N}$ & -13.5 & 4.4 & 1 . \\
\hline N & 13 & -4.4 & 1.7 \\
\hline $\mathrm{N}$ & -13.5 & -4.4 & -1 \\
\hline $\mathrm{N}$ & 15.3 & 2.7 & 0.8 \\
\hline N & $-15 \cdot 3$ & -2.75 & 0. \\
\hline & -15 & 2 . & $3-0$ \\
\hline $\mathrm{N}$ & 15.3 & -2.7 & -0.86 \\
\hline C & 5.2 & & -0 \\
\hline $\mathrm{C}$ & -5.262 & 3.90 & 0.4 \\
\hline C & 5 . & -3.90 & 0 . \\
\hline $\mathrm{C}$ & -5.26 & -3.905007 & -0.48 \\
\hline $\mathrm{C}$ & 8.25 & 5.16 & $-2 \cdot 4$ \\
\hline$C$ & -8.25 & 5.16 & 2.41 \\
\hline $\mathrm{C}$ & 8 . & -5.162339 & 2.4 \\
\hline C & -8.25 & -5.162339 & -2.41 \\
\hline$\Omega$ & 13.18 & 4.594298 & 0.56 \\
\hline & $-13.1 \varepsilon$ & 4.594298 & -0.569204 \\
\hline 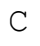 & 13.18 & -4.59 & -0.5 \\
\hline$\Omega$ & -13.1 & -4.594298 & 0.56 \\
\hline $\mathrm{C}$ & 15.50 & 2.43 & -1.472 \\
\hline 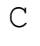 & -15.502574 & -2.438644 & -1.472010 \\
\hline 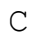 & -15.5 & 2.438644 & 1.47 \\
\hline S & 15.502574 & -2.438644 & 1.472010 \\
\hline $\mathrm{H}$ & 5.5 & 4.0 & 0.541 \\
\hline $\mathrm{H}$ & 7.870 & 5.181281 & -3.428 \\
\hline $\mathrm{H}$ & 13.56 & 4.48 & 1.579 \\
\hline $\mathrm{H}$ & 15.41 & 2.824545 & -2.480138 \\
\hline $\mathrm{H}$ & 15.41 & -2.824545 & 2.480 \\
\hline $\mathrm{H}$ & 13.56 & $-4 \cdot 4$ & -1.57 \\
\hline $\mathrm{H}$ & 7.8 & -5.181281 & 3.428 \\
\hline & 5.5 & -4.059978 & -0.541 \\
\hline
\end{tabular}




\begin{tabular}{|c|c|c|c|}
\hline & -5.581513 & 978 & -0.541586 \\
\hline & -7.870424 & 5.181281 & 3.428091 \\
\hline & -13.566307 & 4.484955 & 135 \\
\hline & -15.410832 & 2.824545 & 2.4801 \\
\hline & -15.410832 & -2.824545 & -2.4801 \\
\hline & -13.566307 & -4.484955 & 1.579135 \\
\hline & -7.870424 & -5.181281 & -3.428091 \\
\hline & -5.581513 & -4.059978 & 0.541586 \\
\hline & 1.882796 & -1.6 & 1.130953 \\
\hline & 1.882796 & 1.606569 & -1.130953 \\
\hline & -1.882796 & 1.606569 & 1.130953 \\
\hline & -1.882796 & -1.606569 & -1.130953 \\
\hline & 2.278641 & -1.954560 & 3.489435 \\
\hline & -2.278641 & 1.954560 & 3.489435 \\
\hline & 2.27 & 560 & -3.4 \\
\hline & -2.2 & -1.9 & -3.4 \\
\hline & 4.132922 & -3.240910 & 0.68302 \\
\hline & -4.132922 & 3.240910 & 0 . \\
\hline & 4.132922 & 910 & -0 . \\
\hline & -4.13 & -3.240910 & -0.6 \\
\hline & 9.5 & -5.3 & 2.2 \\
\hline & -9.558098 & 5.345513 & 2.25 \\
\hline & 9.558098 & 5.345513 & -2.250011 \\
\hline & -9.5 & -5.3 & -2.25 \\
\hline & 5 & -3.5 & 3. \\
\hline C & 345 & 3.5 & -3. \\
\hline$C$ & -4.486345 & 3.5 & 3.0 \\
\hline$C$ & -4.486345 & -3.5 & -3.005860 \\
\hline 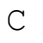 & 9.157188 & -5.2 & -0.08673 \\
\hline 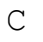 & 9.15 & 5.2 & \\
\hline$\tau$ & -9.1 & 5.2 & -0 . \\
\hline & -9.1 & -5.2 & 0 . \\
\hline 4 & 2.164954 & -1.821478 & 0.1 \\
\hline $\mathrm{H}$ & -2.164954 & 1.821478 & 0.108302 \\
\hline 4 & -2.164954 & -1.821478 & -0.108302 \\
\hline 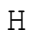 & 2.1 & 1.821 & -0 . \\
\hline$\sqrt{ }$ & 11 & $-5 . c$ & -0. \\
\hline & -11.9 & 014 & -0 \\
\hline & 11.961613 & 5.065014 & 0.416764 \\
\hline & -11.961613 & $3-5.065014$ & 0.416764 \\
\hline N & 15.990272 & -1.218111 & 1.319265 \\
\hline$\sqrt{ }$ & -15 & -1.218111 & -1.319265 \\
\hline N & 15.9 & 1.218111 & -1.3192 \\
\hline & -15.990272 & 1.218111 & 1 . \\
\hline $\mathrm{C}$ & 12.339934 & -4.985759 & 1.92 \\
\hline & 12.339934 & 4.985759 & -1.923420 \\
\hline C & -12.339934 & 985759 & .923420 \\
\hline $\mathrm{C}$ & -12.339934 & $4-4.985759$ & -1.923420 \\
\hline $\mathrm{C}$ & 15.8 & -1.579809 & -1.020204 \\
\hline C & 15.889480 & 1.579809 & 1.020204 \\
\hline & -15.889480 & -1.579809 & 1.020204 \\
\hline $\mathrm{C}$ & -15.889480 & 1.579809 & -1.02020 \\
\hline $\mathrm{H}$ & 9.530062 & 5.266767 & 1.102432 \\
\hline $\mathrm{H}$ & 12.008262 & 5.204969 & -2.931213 \\
\hline $\mathrm{H}$ & 16.117159 & 1.254807 & 2.02 \\
\hline 11 & 16.117159 & -1.254807 & -2.02831 \\
\hline & 12.008262 & -5.204969 & 2.9312 \\
\hline
\end{tabular}

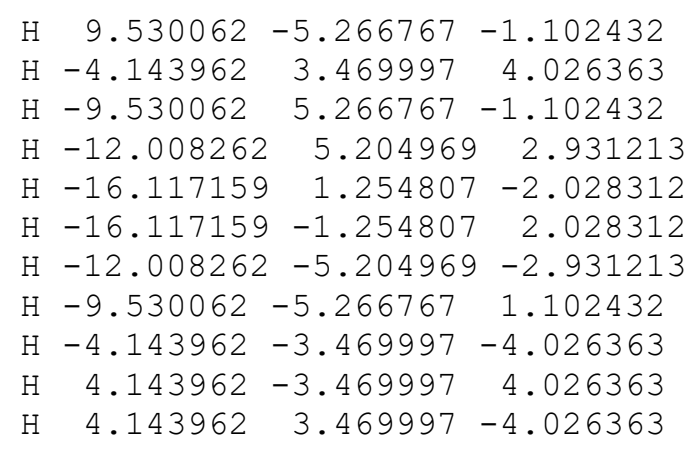

\section{$\Delta \mathrm{H}=-5303.518954$}

\section{$7 \mathrm{CC}-4$}

$\begin{array}{lrrr}\text { C } & -8.518040 & 2.191783 & 1.600921 \\ \text { C } & -10.275861 & 0.621358 & 0.400416 \\ \text { C } & -7.092667 & 2.380595 & 1.940151 \\ \text { C } & -4.499631 & 1.846751 & 2.297684 \\ \text { C } & 8.518040 & -2.191783 & 1.600921 \\ \text { C } & 10.275861 & -0.621358 & 0.400416 \\ \text { C } & 7.092667 & -2.380595 & 1.940151 \\ \text { C } & 4.499631 & -1.846751 & 2.297684 \\ \text { C } & 8.518040 & 2.191783 & -1.600921 \\ \text { C } & 10.275861 & 0.621358 & -0.400416 \\ \text { C } & 7.092667 & 2.380595 & -1.940151 \\ \text { C } & 4.499631 & 1.846751 & -2.297684 \\ \text { C } & -8.518040 & -2.191783 & -1.600921 \\ \text { C } & -10.275861 & -0.621358 & -0.400416 \\ \text { C } & -7.092667 & -2.380595 & -1.940151 \\ \text { C } & -4.499631 & -1.846751 & -2.297684 \\ \text { C } & 3.130230 & -1.300580 & 2.466914 \\ \text { C } & 1.126838 & -0.352446 & 1.583873 \\ \text { C } & 0.684294 & -0.245474 & 2.913260 \\ \text { C } & 1.511859 & -0.645501 & 3.948202 \\ \text { C } & -0.684294 & 0.245474 & 2.913260 \\ \text { C } & -1.126838 & 0.352446 & 1.583873 \\ \text { C } & -3.130230 & 1.300580 & 2.466914 \\ \text { C } & -1.511859 & 0.645501 & 3.948202 \\ \text { H } & 1.197851 & -0.575934 & 4.986169 \\ \text { H } & -1.197851 & 0.575934 & 4.986169 \\ \text { C } & 3.130230 & 1.300580 & -2.466914 \\ \text { C } & 1.126838 & 0.352446 & -1.583873 \\ \text { C } & 0.684294 & 0.245474 & -2.913260 \\ \text { C } & 1.511859 & 0.645501 & -3.948202 \\ \text { C } & -0.684294 & -0.245474 & -2.913260 \\ \text { C } & -1.126838 & -0.352446 & -1.583873 \\ \text { C } & -3.130230 & -1.300580 & -2.466914 \\ \text { C } & -1.511859 & -0.645501 & -3.948202 \\ \text { H } & 1.197851 & 0.575934 & -4.986169 \\ \text { H } & -1.197851 & -0.575934 & -4.986169 \\ \text { C } & -2.366175 & 0.931753 & 1.351843 \\ \text { C } & -2.366175 & -0.931753 & -1.351843 \\ \text { C } & 2.366175 & -0.931753 & 1.351843 \\ \text { C } & 2.366175 & 0.931753 & -1.351843 \\ \text { C } & -5.402668 & -1.873124 & -3.367943\end{array}$




\begin{tabular}{|c|c|c|c|}
\hline & -5.402668 & 873124 & 7943 \\
\hline & 5.402668 & -1.873124 & 3.367943 \\
\hline & 5.402668 & 1.873124 & -3.367943 \\
\hline & -6.157448 & -2.540495 & -0.910223 \\
\hline & -6.157448 & 2.540495 & 0.910223 \\
\hline & 6.157448 & -2.540495 & 0.910223 \\
\hline & 6.157448 & 2.540495 & -0.910223 \\
\hline & -9.316210 & -1.313025 & -2.355039 \\
\hline & -9.316210 & 1.313025 & 5039 \\
\hline & 9.316210 & -1.313025 & 2.355039 \\
\hline & 9.316210 & 1.313025 & -2.355039 \\
\hline & -9.865282 & 1.823943 & -0.207176 \\
\hline & -9.865282 & -1.823943 & 0.207176 \\
\hline & 9.865282 & -1.823943 & -0.207176 \\
\hline & 9.865282 & 1.823943 & 0.207176 \\
\hline & -2 & -1.145421 & -3.729806 \\
\hline & 2.720838 & -1.145421 & 3.729806 \\
\hline & -2.720838 & 1.145421 & 3.729806 \\
\hline & 2.720838 & 1.145421 & -3.729806 \\
\hline & -4.879181 & -2.236997 & -1.080918 \\
\hline & -4.879181 & 997 & 18 \\
\hline & 4.879181 & -2.236997 & 1.080918 \\
\hline & 4.879181 & 2.236997 & -1.080918 \\
\hline & -6.694574 & -2.096472 & -3.181834 \\
\hline & -6.694574 & 2.096472 & 834 \\
\hline & 6.694574 & -2.096472 & 834 \\
\hline & 6.694574 & 2.096472 & -3.181834 \\
\hline & -8.925123 & -2.540566 & -0.381172 \\
\hline & -8.925123 & 2.540566 & 0.381172 \\
\hline & 8.925123 & -2.540566 & 0.381172 \\
\hline & 8.925123 & 2.5 & 1172 \\
\hline & -10.129494 & -0.490123 & -1 \\
\hline & -10.129494 & 0.490123 & 1.71856 \\
\hline & 10.129494 & -0.490123 & 1.718560 \\
\hline & 10.129494 & 0.490123 & -1.718560 \\
\hline & 2.7 & 1.11 & -0.361488 \\
\hline & -2 . & -1.117908 & -0.3 \\
\hline & -2.7 & 1.1 & 88 \\
\hline & 2.760698 & -1.117908 & 0.361488 \\
\hline & 5.068735 & -1.650216 & 4.373900 \\
\hline 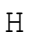 & 6.471770 & -2.850525 & -0.079825 \\
\hline H & 9.193393 & -1.189678 & 3.424319 \\
\hline & 10. & -2.113469 & -1.202236 \\
\hline & 10.1 & 2.1 & 36 \\
\hline & 9.193393 & 1.189678 & -3.424319 \\
\hline & 6.471770 & 2.850525 & 0.079825 \\
\hline 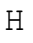 & 5.068735 & 1.650216 & -4.373900 \\
\hline & -5.068735 & -1.650216 & -4.373900 \\
\hline & -6.471770 & -2.850525 & 0.079825 \\
\hline & -9.193393 & -1.189678 & -3.424319 \\
\hline & -10.180448 & -2.113469 & 1.20223 \\
\hline & -9.193393 & 1.189678 & 3.424319 \\
\hline & -10.180448 & 2.113469 & -1.202236 \\
\hline & -6.471770 & 2.850525 & -0.079825 \\
\hline & -5.068735 & 1.650216 & 4.37390 \\
\hline & 0.000000 & 0.000000 & 0.680021 \\
\hline & 0.000000 & 0.000000 & -0.680021 \\
\hline
\end{tabular}

\section{$\Delta \mathrm{H}=-3165.88412$}

\section{CC-5}

$\begin{array}{lrrr}\text { C } & -8.434134 & 2.945027 & 1.797934 \\ \text { C } & -10.901706 & 2.203385 & 1.135618 \\ \text { C } & -6.978177 & 2.795706 & 2.006399 \\ \text { C } & -4.456277 & 1.951669 & 2.284828 \\ \text { C } & 8.434134 & -2.945027 & 1.797934 \\ \text { C } & 10.901706 & -2.203385 & 1.135618 \\ \text { C } & 6.978177 & -2.795706 & 2.006399 \\ \text { C } & 4.456277 & -1.951669 & 2.284828 \\ \text { C } & 8.434134 & 2.945027 & -1.797934 \\ \text { C } & 10.901706 & 2.203385 & -1.135618 \\ \text { C } & 11.821016 & 1.179969 & -0.596748 \\ \text { C } & 6.978177 & 2.795706 & -2.006399 \\ \text { C } & 4.456277 & 1.951669 & -2.284828 \\ \text { C } & 11.821016 & -1.179969 & 0.596748 \\ \text { C } & -8.434134 & -2.945027 & -1.797934 \\ \text { C } & -10.901706 & -2.203385 & -1.135618 \\ \text { C } & -11.821016 & -1.179969 & -0.596748 \\ \text { C } & -6.978177 & -2.795706 & -2.006399 \\ \text { C } & -4.456277 & -1.951669 & -2.284828 \\ \text { C } & -11.821016 & 1.179969 & 0.596748 \\ \text { C } & 3.114034 & -1.339846 & 2.453441 \\ \text { C } & 1.125564 & -0.355465 & 1.580545 \\ \text { C } & 0.683080 & -0.249728 & 2.909829 \\ \text { C } & 1.507413 & -0.664616 & 3.941584 \\ \text { C } & -0.683080 & 0.249728 & 2.909829 \\ \text { C } & -1.125564 & 0.355465 & 1.580545 \\ \text { C } & -3.114034 & 1.339846 & 2.453441 \\ \text { C } & -1.507413 & 0.664616 & 3.941584 \\ \text { H } & 1.197169 & -0.594892 & 4.980688 \\ \text { H } & -1.197169 & 0.594892 & 4.980688 \\ \text { C } & 3.114034 & 1.339846 & -2.453441 \\ \text { C } & 1.125564 & 0.355465 & -1.580545 \\ \text { C } & 0.683080 & 0.249728 & -2.909829 \\ \text { C } & 1.507413 & 0.664616 & -3.941584 \\ \text { C } & -0.683080 & -0.249728 & -2.909829 \\ \text { C } & -1.125564 & -0.355465 & -1.580545 \\ \text { C } & -3.114034 & -1.339846 & -2.453441 \\ \text { C } & -1.507413 & -0.664616 & -3.941584 \\ \text { H } & 1.197169 & 0.594892 & -4.980688 \\ H & -1.197169 & -0.594892 & -4.980688 \\ \text { C } & 5.292246 & -2.162874 & 3.387737 \\ \text { C } & -5.292246 & 2.162874 & 3.387737 \\ \text { C } & 5.292246 & 2.162874 & -3.387737 \\ \text { C } & -5.292246 & -2.162874 & -3.387737 \\ \text { C } & 6.106491 & -2.701287 & 0.916767 \\ \text { C } & -6.106491 & 2.701287 & 0.916767 \\ \text { C } & 6.106491 & 2.701287 & -0.916767 \\ \text { C } & -6.106491 & -2.701287 & -0.916767 \\ \text { C } & 9.349438 & -2.520822 & 2.771367 \\ \text { C } & -9.349438 & 2.520822 & 2.771367 \\ \text { C } & 9.349438 & 2.520822 & -2.771367 \\ \text { C } & -9.349438 & -2.520822 & -2.771367\end{array}$




\begin{tabular}{|c|c|c|c|}
\hline $\mathrm{C}$ & 10.110627 & 2.959959 & -0.257164 \\
\hline C & 10.110627 & -2.959959 & 0.257164 \\
\hline & -10.110627 & 2.959959 & 0.257164 \\
\hline & -10.110627 & $7-2.959959$ & -0.257164 \\
\hline & 2.085694 & -0.011679 & 1.335608 \\
\hline & 12.085694 & $4-0.011679$ & -1.335608 \\
\hline & 12.085694 & 0.011679 & -1.335608 \\
\hline & 12.085694 & 0.011679 & 5608 \\
\hline & 2.358881 & -0.945168 & 1.342398 \\
\hline & -2.358881 & 0.945168 & 1.34 \\
\hline & 2.358881 & 0.945168 & -1.342398 \\
\hline & -2.358881 & -0.945168 & -1.34 \\
\hline & 2.705681 & -1.186348 & 3.71 \\
\hline & -2.705681 & 1.186348 & 3.71 \\
\hline & 2.705681 & 1.186348 & -3.71 \\
\hline & -2.705681 & -1.186348 & -3.71 \\
\hline & 4.864166 & -2.258365 & 1.05 \\
\hline & -4.864166 & 2.258365 & 1.05 \\
\hline & 4.864166 & 2.258365 & -1.05 \\
\hline & -4.864166 & -2.258365 & -1.05 \\
\hline & 6.549 & -2.553519 & 3.24 \\
\hline & -6.549031 & 2.553519 & 3.2 \\
\hline & 6.549031 & 2.553519 & -3.2 \\
\hline & -6.549031 & -2.553519 & -3.24 \\
\hline & 8.864969 & -3.268146 & 0.5 \\
\hline & -8.864969 & 3.268146 & 0.57 \\
\hline & 8 . & 3.2 & -0.5 \\
\hline & -8.864969 & -3.268146 & -0.578047 \\
\hline $\mathrm{N}$ & 10. & -2.100733 & 2.4 \\
\hline $1 N$ & -10.553477 & 2.100733 & 9067 \\
\hline $\mathrm{N}$ & 53477 & 2.10 & -2.4 \\
\hline & -10.553477 & $7-2.100733$ & -2.419067 \\
\hline & 12.001061 & -1.154792 & -0.7 \\
\hline N & 12.001061 & 1.154792 & 0.72 \\
\hline $\mathrm{N}$ & -12.001061 & 1.154792 & -0 \\
\hline . & -12.001061 & $L-1.154792$ & 3346 \\
\hline $\mathrm{M}$ & 2.750428 & -1.128661 & 0.35 \\
\hline $\mathrm{H}$ & -2.750428 & 1.128661 & 0.35 \\
\hline 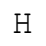 & -2.750428 & -1.128661 & -0.35 \\
\hline $\mathrm{H}$ & 2.750428 & 1.128661 & -0.35 \\
\hline 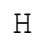 & 4.933085 & -1.971070 & 4.35 \\
\hline $\mathrm{H}$ & 6.445124 & -2.947459 & -0.082996 \\
\hline $\mathrm{H}$ & 9.062946 & -2.428123 & 3.81 \\
\hline $\mathrm{H}$ & 10.452473 & -3.211251 & -0.739855 \\
\hline $\mathrm{H}$ & 12.218 & -0.017851 & 2.41 \\
\hline $\mathrm{H}$ & 12.218040 & 0.017851 & -2.410574 \\
\hline $\mathrm{H}$ & 10.452473 & 3.211251 & 0.7 \\
\hline II & 9.062946 & 2.428123 & -3.812164 \\
\hline $\mathrm{H}$ & 6.445124 & 2.947459 & 0.082996 \\
\hline $\mathrm{H}$ & 4.933085 & 1.971070 & -4.391150 \\
\hline $\mathrm{H}$ & -4.933085 & -1.971070 & -4.391150 \\
\hline $\mathrm{H}$ & -6.445124 & -2.947459 & 0.082996 \\
\hline $\mathrm{H}$ & -9.062946 & -2.428123 & -3.812164 \\
\hline $\mathrm{H}$ & -10.452473 & $3-3.211251$ & 9855 \\
\hline $\mathrm{H}$ & -12.218040 & -0.017851 & -2.410574 \\
\hline $\mathrm{H}$ & -12 & 51 & 74 \\
\hline & 452473 & 1251 & 39855 \\
\hline
\end{tabular}

$\begin{array}{rrrr}\mathrm{H} & -9.062946 & 2.428123 & 3.812164 \\ \mathrm{H} & -6.445124 & 2.947459 & -0.082996 \\ \mathrm{H} & -4.933085 & 1.971070 & 4.391150 \\ \mathrm{C} & 0.000000 & 0.000000 & 0.679332 \\ \mathrm{C} & 0.000000 & 0.000000 & -0.679332\end{array}$

$\Delta \mathrm{H}=-3691.967736$

\section{CC- 6}

\begin{tabular}{|c|c|c|c|}
\hline $\mathrm{C}$ & -8.318620 & 3.376734 & 1.837958 \\
\hline $\mathrm{C}$ & -10.068668 & 3.661314 & 0.415040 \\
\hline $\mathrm{C}$ & -10.954940 & 3.303393 & 1.438992 \\
\hline C & -9.218110 & 3.271966 & 2.905803 \\
\hline C & -12.277302 & 2.728183 & 1.115921 \\
\hline C & -6.892658 & 3.018933 & 2.002890 \\
\hline C & -5.229647 & 2.312717 & $3.37^{\circ}$ \\
\hline $\mathrm{C}$ & -4.434836 & 1.997251 & 2.26 \\
\hline $\mathrm{C}$ & -6.069769 & 2.777683 & 0.89 \\
\hline $\mathrm{C}$ & -12.935748 & 1.887056 & 2.0 \\
\hline C & -13.747392 & 0.690708 & 0.2 \\
\hline $\mathrm{C}$ & -13.45 & 1.770755 & -0.5 \\
\hline $\mathrm{H}$ & -10.427672 & 3.872962 & -0.5 \\
\hline $\mathrm{H}$ & -8.862162 & 3.176358 & 3.92 \\
\hline $\mathrm{H}$ & -4.862694 & 2.138077 & 4.38 \\
\hline $\mathrm{H}$ & -6.420 & 2.986 & -0.10 \\
\hline $\mathrm{H}$ & -12.8 & 1.99 & \\
\hline $\mathrm{H}$ & -13.752784 & 1.780604 & $-1 \cdot 6$ \\
\hline $\mathrm{C}$ & & -3.376734 & 1.83 \\
\hline C & 10.068668 & -3.66 & 0.41 \\
\hline C & 10 . & -3.30 & 1.4 \\
\hline $\mathrm{C}$ & 9.218110 & -3.27 & 2.90 \\
\hline $\mathrm{C}$ & 12.277 & -2.72 & 1.1 \\
\hline ه & 6.892658 & -3.018 & 2.00 \\
\hline$C$ & 5.229647 & -2.31 & 3.3 \\
\hline $\mathrm{C}$ & 4.434836 & -1.997 & 2.26 \\
\hline $\mathrm{C}$ & 6.069769 & $-2.77^{\prime}$ & 0.8 \\
\hline $\mathrm{C}$ & 12.935748 & -1.887 & 2.02 \\
\hline $\mathrm{C}$ & 13.747 & -0.69 & 0.2 \\
\hline $\mathrm{C}$ & 13.454654 & -1.770755 & $-0.5 \varepsilon$ \\
\hline $\mathrm{H}$ & 10.427 & -3.872 & -0.58 \\
\hline $\mathrm{H}$ & 8.862162 & -3.176358 & 3.92 \\
\hline $\mathrm{H}$ & 4.862694 & -2.138077 & 4.38 \\
\hline $\mathrm{H}$ & 6.420307 & -2.986606 & -0.10 \\
\hline $\mathrm{H}$ & 12.813401 & -1.994460 & 3.09 \\
\hline $\mathrm{H}$ & 13.752784 & -1.780604 & $-1.62 \varepsilon$ \\
\hline $\mathrm{C}$ & 8.318620 & 3.376 & -1.83 \\
\hline $\mathrm{C}$ & 10.068668 & 3.661314 & -0.41 \\
\hline $\mathrm{C}$ & 10.954940 & 3.30 & $-1 \cdot 4$ \\
\hline $\mathrm{C}$ & 9.218110 & 3.271966 & -2.90 \\
\hline $\mathrm{C}$ & 12.277302 & 2.728 & $-1 \cdot 1$ \\
\hline $\mathrm{C}$ & 6.892658 & 3.018933 & -2.00289 \\
\hline $\mathrm{C}$ & 5.229647 & 2.312 & -3.377079 \\
\hline $\mathrm{C}$ & 4.434836 & 1.997251 & -2.26 \\
\hline $\mathrm{C}$ & 6.0 & 2.777683 & -0.8 \\
\hline $\mathrm{C}$ & 12 . & 1. & -2 \\
\hline 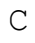 & 13. & 0.690708 & -0.2 \\
\hline
\end{tabular}




\begin{tabular}{|c|c|c|c|}
\hline & 13.454654 & 1.770755 & 7493 \\
\hline & 10.427672 & 3.872962 & 0.585247 \\
\hline & 8.862162 & 3.176358 & -3.924617 \\
\hline & 4.862694 & 2.138077 & -4.380744 \\
\hline & 6.420307 & 2.986606 & 0.104948 \\
\hline & 12.813401 & 1.994460 & -3.097545 \\
\hline & 13.752784 & 1.780604 & 1.628874 \\
\hline & -8.318620 & -3.376734 & -1.837958 \\
\hline & -10 & & \\
\hline & -10.954940 & -3.303393 & $3-1.4389$ \\
\hline & -9.218110 & -3.271966 & -2.905803 \\
\hline & -12.277302 & & $3-1.115921$ \\
\hline & -6.892658 & -3.018933 & -2.002890 \\
\hline & -5.229647 & -2.312717 & -3.377079 \\
\hline & -4.434836 & -1.997251 & -2.269213 \\
\hline & 769 & -2 . & -0.89906 \\
\hline & -12.935748 & $3-1.887056$ & -2.02660 \\
\hline & -13.747392 & -0.690708 & -0.261845 \\
\hline & -13.454654 & -1.770755 & 0.587493 \\
\hline & -10.427672 & -3.872962 & 0.585247 \\
\hline & -8.862162 & -3.176358 & -3.924617 \\
\hline & -4.862694 & -2.138077 & -4.380744 \\
\hline & -6.420307 & -2.986606 & 0.104948 \\
\hline & -12.813401 & -1.994460 & -3.097545 \\
\hline & 52784 & -1.780604 & 1.628874 \\
\hline & 3.109396 & -1.351014 & 2.443799 \\
\hline & 2.356109 & -0.946331 & 1.336090 \\
\hline & 1.125233 & -0.354192 & 1.578707 \\
\hline & 0.683620 & -0.249481 & 2.908323 \\
\hline & 1.510010 & -0.667555 & 3.937445 \\
\hline & -0.683620 & 9481 & 3323 \\
\hline & -1.125233 & 0.3 & 707 \\
\hline & -2.356109 & 0.946331 & 1.336090 \\
\hline & -3.109396 & 1.351014 & 2.443799 \\
\hline & -1.510010 & 0.667555 & 3.937445 \\
\hline & 2.744505 & -1.129391 & 0.342830 \\
\hline & 1.203760 & -0.596896 & 4.977672 \\
\hline & -2.744505 & 1.129391 & 0.3 \\
\hline & -1.203760 & 0.596896 & 4.977672 \\
\hline & 3.109396 & 1.351014 & -2.443799 \\
\hline & 2.356109 & 0.946331 & -1.336090 \\
\hline & 1.125233 & 0.354192 & -1.578707 \\
\hline & 0.683620 & 0.249481 & -2.908323 \\
\hline 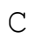 & 1.51 & 0.6 & -3. \\
\hline & -0.683620 & -0.249481 & -2.908323 \\
\hline & -1.125233 & -0.354192 & -1.578707 \\
\hline & -2.356109 & -0.946331 & -1.336090 \\
\hline & -3.109396 & -1.351014 & -2.443799 \\
\hline & -1.510010 & -0.667555 & -3.937445 \\
\hline & 2.744505 & 1.129391 & -0.342830 \\
\hline & 1.203760 & 0.596896 & -4.977672 \\
\hline & -2.744505 & -1.129391 & -0.342830 \\
\hline & -1.203760 & -0.596896 & -4.977672 \\
\hline & 2.704155 & 1.197159 & -3.708980 \\
\hline & -2.704155 & -1.197159 & -3.708980 \\
\hline & -2.704155 & 1.197159 & 3.708980 \\
\hline & 2.704155 & -1.197159 & 3.708980 \\
\hline
\end{tabular}

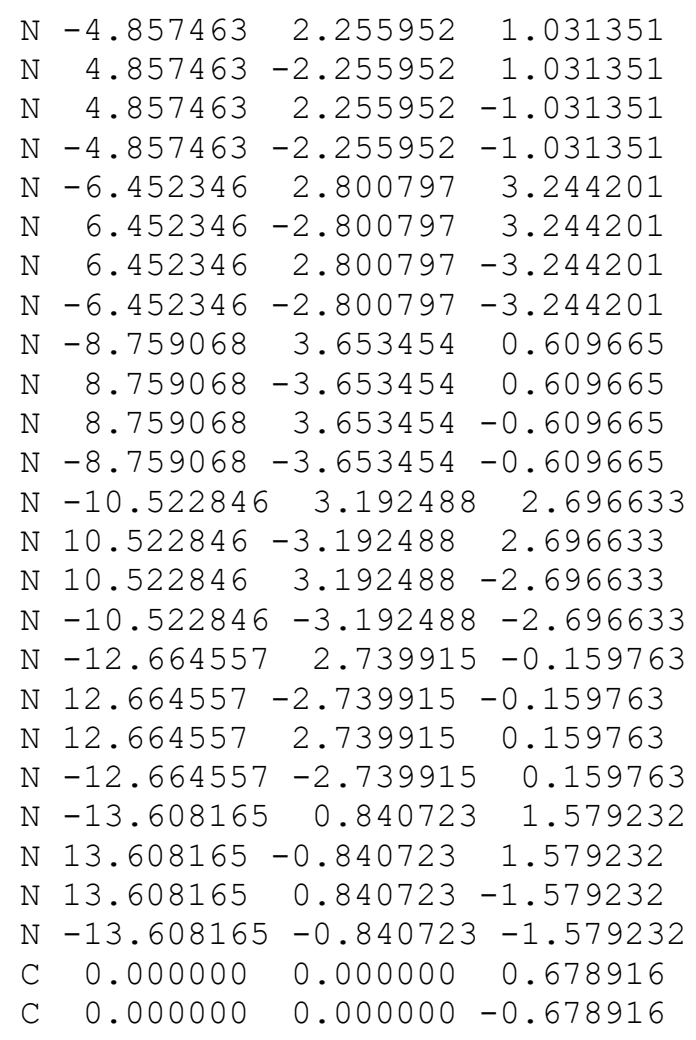

$\Delta \mathrm{H}=-4218.049902$

\section{CC-7}

$\begin{array}{lrcc}\text { C } & -8.224789 & 3.628797 & 1.824069 \\ \text { C } & -10.851694 & 4.005190 & 1.525353 \\ \text { C } & -12.301007 & 3.806071 & 1.310678 \\ \text { C } & -6.836257 & 3.141215 & 1.980543 \\ \text { C } & -4.425786 & 2.013554 & 2.255877 \\ \text { C } & -14.544278 & 2.482304 & 0.763702 \\ \text { C } & 8.224789 & -3.628797 & 1.824069 \\ \text { C } & 10.851694 & -4.005190 & 1.525353 \\ \text { C } & 12.301007 & -3.806071 & 1.310678 \\ \text { C } & 6.836257 & -3.141215 & 1.980543 \\ \text { C } & 4.425786 & -2.013554 & 2.255877 \\ \text { C } & 14.544278 & -2.482304 & 0.763702 \\ \text { C } & 8.224789 & 3.628797 & -1.824069 \\ \text { C } & 10.851694 & 4.005190 & -1.525353 \\ \text { C } & 12.301007 & 3.806071 & -1.310678 \\ \text { C } & 6.836257 & 3.141215 & -1.980543 \\ \text { C } & 4.425786 & 2.013554 & -2.255877 \\ \text { C } & 14.544278 & 2.482304 & -0.763702 \\ \text { C } & -8.224789 & -3.628797 & -1.824069 \\ \text { C } & -10.851694 & -4.005190 & -1.525353 \\ \text { C } & -12.301007 & -3.806071 & -1.310678 \\ \text { C } & -6.836257 & -3.141215 & -1.980543 \\ \text { C } & -4.425786 & -2.013554 & -2.255877 \\ \text { C } & -14.544278 & -2.482304 & -0.763702 \\ \text { C } & 3.110086 & -1.349650 & 2.437438 \\ \text { C } & 1.125354 & -0.351424 & 1.577792\end{array}$




\begin{tabular}{|c|c|c|c|}
\hline & 717 & -0.247588 & 07833 \\
\hline & 1.514686 & -0.664164 & 3.935018 \\
\hline & -0.684717 & 0.247588 & 2.907833 \\
\hline & -1.125354 & 0.351424 & 1.577792 \\
\hline & -3.110086 & 1.349650 & 2.437438 \\
\hline & -1.514686 & 0.664164 & 3.935018 \\
\hline & 1.211625 & -0.592711 & 4.97611 \\
\hline & -1.211625 & 0.592711 & 4.976119 \\
\hline & 3.110086 & 1.349650 & -2.437438 \\
\hline & 1.125354 & 0.351424 & -1.577792 \\
\hline & 0.684717 & 0.247588 & -2.90783 \\
\hline & 1.514686 & 0.664164 & -3.93501 \\
\hline & -0.684717 & -0.247588 & -2.90783 \\
\hline & -1.125354 & 1424 & -1.57779 \\
\hline & -3.110086 & -1.349650 & -2.437438 \\
\hline & -1.514686 & -0.664164 & -3.9 \\
\hline & 1.211625 & 0.592711 & -4.97611 \\
\hline & -1.211625 & -0.592711 & -4.9761 \\
\hline & -15.299438 & $3-1.270358$ & $8-0.384429$ \\
\hline & -15.29 & 1.270358 & 429 \\
\hline & 15.29 & -1.270358 & 0.3 \\
\hline & 15.299438 & 1.270358 & -0.384429 \\
\hline & 2.707964 & -1.195161 & 3.70 \\
\hline & -2.7 & 1.195161 & 3.7 \\
\hline & 2.707964 & 1.195161 & -3.703589 \\
\hline & -2.707964 & $-1 \cdot 1$ & -3 \\
\hline & 2.35 & -0.942448 & 1. \\
\hline & 2.355667 & 0.942448 & -1.332167 \\
\hline 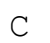 & -2.355667 & 0.942448 & 1.332167 \\
\hline & -2.355667 & -0.942448 & -1.332167 \\
\hline & 140 & -2.3 & 3.360636 \\
\hline & 5.199 & 2.386560 & -3.360636 \\
\hline & -5.199140 & 2.386560 & 3.360636 \\
\hline & -5.199140 & -2.386560 & -3.360636 \\
\hline 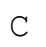 & 6.043672 & -2.814808 & 0.876920 \\
\hline C & 6.043672 & 2.814808 & -0.876920 \\
\hline $\mathrm{C}$ & -6.0 & 2.814808 & 6920 \\
\hline & -6.0 & -2.8 & -0.8 \\
\hline$r$ & 9.082 & -3.732430 & 2.924663 \\
\hline $\mathrm{C}$ & 9.082707 & 3.732430 & -2.924663 \\
\hline $\mathrm{C}$ & -9.082707 & 3.732430 & 2.92466 \\
\hline $\mathrm{C}$ & -9.082707 & -3.732430 & -2.924663 \\
\hline C & 9.969538 & -4.085150 & 0.442121 \\
\hline C & 9.9 & 4.0 & -0.442121 \\
\hline C & -9.969538 & 4.085150 & 0.4 \\
\hline C & -9.969538 & -4.085150 & -0.442121 \\
\hline 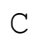 & 13.123631 & -3.318244 & 2.334728 \\
\hline $\mathrm{C}$ & 13.123631 & 3.318244 & -2.334728 \\
\hline C & -13.123631 & 3.318244 & 2.334728 \\
\hline C & $-13 \cdot 123631$ & -3.318244 & $4-2.33472$ \\
\hline & & -3.257714 & -0.212399 \\
\hline & 13.902408 & 3.257714 & 0.212399 \\
\hline C & -13.902408 & 3.257714 & -0.21239 \\
\hline $\mathrm{C}$ & -13.902408 & $3-3.257714$ & 0.212399 \\
\hline 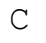 & 15.512322 & 0.238793 & -1.312249 \\
\hline C & 15.5123 & -0.238793 & 1.31224 \\
\hline & & & \\
\hline
\end{tabular}

\begin{tabular}{|c|c|c|c|}
\hline & to. & 0.238793 & \\
\hline & 852944 & -2.246550 & 1.014364 \\
\hline & -4.852944 & 2.246550 & 1.01 \\
\hline & & & \\
\hline & -4 . & -2.2 & -1 . \\
\hline & & -2.9 & \\
\hline & 08 & 2.9 & 3. \\
\hline & 6108 & 2.933419 & -3.22376 \\
\hline & -6 & -2 & -3 \\
\hline & & -3 & \\
\hline & -8 . & & \\
\hline & 264 & & -0 . \\
\hline & -8 & $-3 . \varepsilon$ & -0 . \\
\hline & 10. & -3.8 & 2. \\
\hline & -10 & 6597 & 2 . \\
\hline & 10. & $3 . \varepsilon$ & -2 \\
\hline & -10 & & \\
\hline & 12 & -3.86 & 0.05 \\
\hline & -12 & $4 \quad 3.8$ & 9665 \\
\hline & & 3.8 & -0.0 \\
\hline & -12 & -3 & -0 \\
\hline & 14. & -2.6 & 2.0 \\
\hline & -14 & $\$ 42$ & 2. \\
\hline & 14. & & -2 \\
\hline & -14 & -2 . & -2 \\
\hline & & & -0 . \\
\hline & -1 & -1 & \\
\hline & -15 & 1. & -0 \\
\hline & 15. & 1.0 & 0. \\
\hline & 2 . & 1.1 & -0 . \\
\hline & -2 & -1.1 & -0 . \\
\hline & -2 & 1.1 & \\
\hline & 2 . & -1 & \\
\hline & 4. & -2.2 & 4. \\
\hline & 6. & -3.0 & -0 \\
\hline & 8. & -3. & 3. \\
\hline & 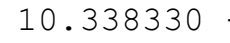 & -4 & -0 . \\
\hline & & -3 & \\
\hline & 14 & -3.2 & -1 \\
\hline & 15. & -0.4 & 2. \\
\hline & 15. & 0.4 & -2.3 \\
\hline & 14. & & 1. \\
\hline 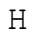 & & & -3 \\
\hline & 10 & & \\
\hline & 8 . & 3. & -3 \\
\hline & 6. & 3.0 & 0 . \\
\hline $\mathrm{H}$ & 4.8 & 2.2 & -4 \\
\hline $\mathrm{H}$ & -4 & 2.2 & \\
\hline I & -6.3 & 3.0 & -0.1 \\
\hline & -10 & 4. & -0 . \\
\hline & & 3.6 & \\
\hline & -14. & 3.2 & -1 \\
\hline & 12.8 & & \\
\hline & & & \\
\hline & -15.6 & 318 & 2.3 \\
\hline & -12.8 & -3. & -3 \\
\hline & & & \\
\hline
\end{tabular}


$\begin{array}{cccc}\mathrm{H} & -8.701259 & -3.631643 & -3.933611 \\ \mathrm{H} & -10.338330 & -4.268454 & 0.560191 \\ \mathrm{H} & -4.833336 & -2.218900 & -4.365920 \\ \mathrm{H} & -6.397491 & -3.003857 & 0.129796 \\ \mathrm{C} & 0.000000 & 0.000000 & 0.678681 \\ \mathrm{C} & 0.000000 & 0.000000 & -0.678681\end{array}$

\section{$\Delta \mathrm{H}=-4744.129467$}

\section{$7 \mathrm{CC}-8$}

\begin{tabular}{|c|c|c|c|}
\hline C & -8.165857 & 3.762642 & 1.806441 \\
\hline $\mathrm{C}$ & -10.738364 & 4.435195 & 1.544217 \\
\hline & -12.206306 & 4.506498 & 1.376310 \\
\hline & -6.808250 & 3.194026 & 1.963283 \\
\hline & -4.427284 & 2.007463 & 2.248230 \\
\hline & -14.764003 & 3.850642 & 1.005035 \\
\hline & 8.165857 & -3.762642 & 1.8 \\
\hline & 10.738364 & -4.435195 & 1.54 \\
\hline & 12.206306 & -4.506498 & 1.3 \\
\hline & 6.808250 & -3.194026 & 1.96 \\
\hline & 4.427284 & -2.007463 & 2.2 \\
\hline & 14.764003 & -3.850642 & 1.00 \\
\hline & 8.165857 & 3.762642 & -1.8 \\
\hline & 10.738364 & 4.435195 & -1.5 \\
\hline & 12.206 & 4.506498 & $-1 \cdot 3$ \\
\hline & 6.808250 & 3.194026 & -1.96 \\
\hline & 4.427284 & 2.007463 & -2.2 \\
\hline & 14.764003 & 3.850642 & -1.00 \\
\hline & -8.165 & -3.762642 & $-1 \cdot 8$ \\
\hline 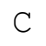 & -10.738364 & -4.435195 & -1.544217 \\
\hline & $-12 \cdot 20$ & -4.506498 & $3-1.3$ \\
\hline 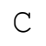 & -6.808250 & -3.194026 & -1.963283 \\
\hline & -4.427 & -2.007463 & -2.24 \\
\hline . & -14.764003 & -3.850642 & -1 \\
\hline $\mathrm{C}$ & 3.114978 & -1.338382 & 2.43 \\
\hline C & 1.126068 & -0.347514 & $1.57^{\circ}$ \\
\hline $\mathrm{C}$ & 0.686094 & -0.244447 & 2.9 \\
\hline $\mathrm{C}$ & 1.519962 & -0.656091 & 3.93 \\
\hline C & -0.686094 & 0.244447 & 2.907834 \\
\hline $\mathrm{C}$ & -1.126068 & 0.347514 & 1.577494 \\
\hline 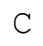 & -3.114978 & 1.338382 & 2.43 \\
\hline C & -1.519962 & 0.656091 & 3.93 \\
\hline $\mathrm{H}$ & 1.218772 & -0.584120 & 4.97 \\
\hline $\mathrm{H}$ & -1.218772 & 0.584120 & 4.97 \\
\hline $\mathrm{C}$ & 3.114978 & 1.338382 & -2.43 \\
\hline & 1.126068 & 0.347514 & -1.57 \\
\hline C & 0.686 & 0.244447 & -2.907834 \\
\hline$C$ & 1.519962 & 0.656091 & -3.934090 \\
\hline C & -0.686094 & -0.244447 & -2.907834 \\
\hline $\mathrm{C}$ & -1.126068 & -0.347514 & -1.5774 \\
\hline $\mathrm{C}$ & -3.114978 & -1.338382 & -2.434551 \\
\hline $\mathrm{C}$ & -1.519962 & -0.656091 & -3.9 \\
\hline & 1.218772 & 0.584120 & -4.975693 \\
\hline & -1.218772 & -0.584120 & -4.9 \\
\hline & -15.947 & -3.003865 & $5-0.745710$ \\
\hline & -17 & -0 . & \\
\hline
\end{tabular}

\begin{tabular}{|c|c|c|c|}
\hline C & 17.179288 & 0.719190 & -0.168842 \\
\hline C & 15.947356 & 3.003865 & $\$ 5710$ \\
\hline C & -15.947356 & 3.00 & 0.745710 \\
\hline & 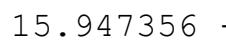 & -3.0 & 0.745710 \\
\hline $\mathrm{C}$ & 17.179288 & 0.719190 & 0.168842 \\
\hline & 7.179288 & -0.719190 & 0.168842 \\
\hline & 6.371039 & 2.989321 & -3.2 \\
\hline & 6.371039 & 2.989321 & 3.20 \\
\hline & 6.371039 & -2.98 & 3.2 \\
\hline & 6.371 & -2.989321 & -3.20 \\
\hline & 8.61 & 3.978 & -0 \\
\hline & -8.61 & 3.978789 & 0.56 \\
\hline & & -3.97 & 0. \\
\hline & -8.61 & -3.978789 & -0.56 \\
\hline & 14.32 & 3.93 & -2.2 \\
\hline & $-14 \cdot 3$ & 3.93 & 2.2 \\
\hline & 4.32 & -3.930 & 2.2 \\
\hline & -14.32 & -3.93 & -2 \\
\hline & 16.29 & 2.79 & 0.5 \\
\hline & 6.2 & -2.79 & 20 \\
\hline & -16.2 & 2.7 & -0 . \\
\hline & 0 & -2.794 & -0.52 \\
\hline $\mathrm{C}$ & 6. & 2.81 & $-0 . \varepsilon$ \\
\hline C & -6 & 2.81 & 0 . \\
\hline C & 6.03 & -2.81 & 0.8 \\
\hline C & -6 . & -2.81 & -0 \\
\hline C & 8.98 & 3.99 & -2.9 \\
\hline C & -8 & & 2.9 \\
\hline C & 8.98 & -3.995 & 2.9 \\
\hline C & -8 & -3. & -2.9 \\
\hline C & 13.96 & 4.313 & 0.04 \\
\hline $\mathrm{C}$ & -1 & 4.31 & -0.0 \\
\hline C & 13. & $-4 \cdot 3$ & -0.0 \\
\hline $\mathrm{C}$ & -13 & -4 & 677 \\
\hline C & 16.49 & 2.20 & -1.756623 \\
\hline C & -16.4 & -2.2 & -1.7 \\
\hline C & -16.4 & 2.20 & 6623 \\
\hline C & 16.49 & -2.204 & 1.75 \\
\hline $\mathrm{H}$ & 6.38 & 2.998 & 0.1 \\
\hline $\mathrm{H}$ & 8.59 & 3.88 & -3.92 \\
\hline & 14.356 & 4.373 & 1.05 \\
\hline $\mathrm{H}$ & 16.38 & 2.45 & -2.8 \\
\hline & 16.38 & -2.45 & 2.8 \\
\hline $\mathrm{H}$ & 14.35 & -4.37 & -1.0 \\
\hline $\mathrm{H}$ & 8.592 & -3.886 & 3.92 \\
\hline 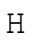 & 6.38 & -2.99 & -0.1 \\
\hline $\mathrm{H}$ & -6.388 & 2.998 & -0.14 \\
\hline $\mathrm{H}$ & -8.59 & 3.88 & 3.92 \\
\hline $\mathrm{H}$ & -14.35 & 4.373589 & -1.05 \\
\hline $\mathrm{H}$ & -16.38 & 2.4 & 3837 \\
\hline $\mathrm{H}$ & -16.38 & -2.459342 & -2.8 \\
\hline 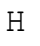 & -14.35 & -4.37 & 1.059324 \\
\hline 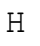 & -8.59 & -3.886 & -3.92 \\
\hline 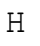 & -6.388 & -2.998235 & 0.146086 \\
\hline$G$ & 2.357 & -0.934 & 1.33 \\
\hline $\mathrm{C}$ & $2.357^{\circ}$ & 0.934437 & -1.33 \\
\hline $\mathrm{C}$ & -2.3577 & 0.93 & 1.3 \\
\hline
\end{tabular}




\begin{tabular}{|c|c|c|c|}
\hline $\mathrm{C}$ & -2.357703 & -0.934437 & -1.330551 \\
\hline & 2.714814 & -1.183467 & 3.701263 \\
\hline & -2.714814 & 1.183467 & 3.701263 \\
\hline & 2.714814 & 1.183467 & -3.70 \\
\hline & -2.714814 & -1.183467 & -3.701263 \\
\hline & 4.856047 & -2.226396 & 1.004647 \\
\hline & -4.856047 & 2.226396 & 1.004647 \\
\hline & 4.856047 & 2.226396 & -1.004647 \\
\hline & -4.856047 & -2.226396 & -1.004647 \\
\hline & 10.263926 & -4.305576 & 2.784961 \\
\hline & -10.263926 & 4.305576 & 2.784961 \\
\hline & 10.263926 & 4.305576 & -2.784961 \\
\hline & -10.263926 & -4.305576 & $5-2.784961$ \\
\hline & 5.190553 & -2.409033 & 3.34 \\
\hline 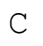 & 5.190553 & 2.409033 & -3.349696 \\
\hline $\mathrm{C}$ & -5.19 & 2.40 & 3.34 \\
\hline $\mathrm{C}$ & -5.190553 & -2.409033 & -3.349696 \\
\hline$C$ & 9.887149 & -4.33 & 0.43 \\
\hline $\mathrm{C}$ & 9.887149 & 4.334456 & -0.439206 \\
\hline C & -9.887149 & 4.33 & 0.43 \\
\hline$C$ & -9.88 & -4.33 & -0.43 \\
\hline $\mathrm{H}$ & 2.742349 & -1.118914 & 0.33 \\
\hline $\mathrm{H}$ & -2.74 & 1.11 & 0.33 \\
\hline $\mathrm{H}$ & -2.742349 & -1.118914 & -0.336357 \\
\hline $\mathrm{H}$ & 2.74 & 1.11 & -0.33 \\
\hline $\mathrm{N}$ & 12.68 & -4.597087 & 0.1 \\
\hline $\mathrm{N}$ & -12.6 & 7087 & 29 \\
\hline $\mathrm{N}$ & 12.68 & 4.59 & -0.13 \\
\hline $\mathrm{N}$ & -12.6 & -4.597087 & -0 . \\
\hline $\mathrm{N}$ & 17.05 & -1.044766 & 1.45 \\
\hline N & -17.0 & -1.0 & $5-1$ \\
\hline $\mathbb{N}$ & 17.059451 & 1.044766 & -1.45 \\
\hline $\mathrm{N}$ & -17.0 & 1.044766 & 1. \\
\hline $\mathrm{C}$ & 13.071148 & -4.297197 & 2.456914 \\
\hline C & 13.07 & 4.297197 & -2.45 \\
\hline C & -13.071148 & 4.297197 & 5914 \\
\hline $\mathrm{C}$ & -13.0 & -4.297197 & -2.4 \\
\hline C & 16. & -1.688 & -0.81 \\
\hline $\mathrm{C}$ & 16.954231 & 1.688952 & 0.81 \\
\hline C & -16.954231 & -1.688952 & 3848 \\
\hline $\mathrm{C}$ & -16.954231 & 1.688952 & -0.8 \\
\hline $\mathrm{H}$ & 10.262777 & 4.502767 & 0.56 \\
\hline $\mathrm{H}$ & 12.712175 & 4.353294 & -3.477559 \\
\hline $\mathrm{H}$ & 17.216440 & 1.514731 & 1.855448 \\
\hline $\mathrm{H}$ & 17.216440 & -1.514 & -1.85 \\
\hline $\mathrm{H}$ & 12.712175 & -4.353294 & 3.477559 \\
\hline $\mathrm{H}$ & 10.262777 & -4.502767 & -0.563228 \\
\hline $\mathrm{H}$ & -4.827779 & 2.242982 & 4.356365 \\
\hline $\mathrm{H}$ & -10.262777 & 4.502767 & -0.563228 \\
\hline $\mathrm{H}$ & -12.712175 & 4.353294 & 7559 \\
\hline $\mathrm{H}$ & -17.216440 & 1.514731 & -1.855448 \\
\hline $\mathrm{H}$ & -17.216440 & -1.514731 & 448 \\
\hline $\mathrm{H}$ & -12.712175 & -4.353294 & -3.477559 \\
\hline $\mathrm{H}$ & -10.262777 & -4.502767 & 0.5 \\
\hline $\mathrm{H}$ & -4.827779 & -2.242982 & -4.356365 \\
\hline $\mathrm{H}$ & 4. & -2.2 & \\
\hline 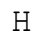 & 4.827779 & 2.242982 & $-4 \cdot 3$ \\
\hline
\end{tabular}

\author{
$\begin{array}{llll}\text { C } & 0.000000 & 0.000000 & 0.678561\end{array}$ \\ C $\quad 0.000000 \quad 0.000000-0.678561$
}

$\Delta \mathrm{H}=-5270.208131$

\section{$8 N N-4$}

\begin{tabular}{|c|c|c|c|}
\hline C & -7.957710 & 2.649977 & 1.029088 \\
\hline $\mathrm{C}$ & -9.533307 & 0.715299 & 0.202502 \\
\hline $\mathrm{C}$ & -6.596879 & 3.044411 & 1.467877 \\
\hline C & -4.036763 & 2.640230 & 2.015631 \\
\hline $\mathrm{C}$ & 7.957710 & -2.649977 & 1.029088 \\
\hline $\mathrm{C}$ & 9.533307 & -0.715299 & 0.202502 \\
\hline $\mathrm{C}$ & 6.596879 & -3.044411 & 1.467877 \\
\hline C & 4.036763 & -2.640230 & 2.015 \\
\hline $\mathrm{C}$ & 7.957710 & 2.649977 & -1.0290 \\
\hline$C$ & 9.533307 & 0.715299 & -0.2025 \\
\hline $\mathrm{C}$ & 6.596879 & 3.044411 & -1.467877 \\
\hline $\mathrm{C}$ & 4.036763 & 2.640230 & -2.0156 \\
\hline C & -7.957710 & -2.649977 & -1.029088 \\
\hline $\mathrm{C}$ & -9.533307 & -0.715299 & -0.2025 \\
\hline $\mathrm{C}$ & -6.596879 & -3.044411 & -1.467877 \\
\hline $\mathrm{C}$ & -4.036763 & -2.640230 & -2.0156 \\
\hline $\mathrm{C}$ & 2.709215 & -2.003602 & 2.2305 \\
\hline C & 0.890380 & -0.710249 & 1.4840 \\
\hline C & 0.561432 & -0.453267 & 2.82 \\
\hline C & 1.323351 & -1.069999 & 3.80 \\
\hline $\mathrm{C}$ & -0.561432 & 0.453267 & 2.8 \\
\hline C & -0.890380 & 0.710249 & 1.48 \\
\hline C & -2.70 & 2.003602 & 2.2 \\
\hline C & -1.323351 & 1.069999 & 3.80919 \\
\hline $\mathrm{N}$ & 0.00 & 0.000000 & 0.6 \\
\hline $\mathrm{H}$ & 1.093347 & -0.941995 & 4.8634 \\
\hline $\mathrm{H}$ & -1.0 & 0.941995 & 4.8 \\
\hline $\mathrm{C}$ & 2.709215 & 2.003602 & -2.2305 \\
\hline $\mathrm{C}$ & 0.890380 & 0.710249 & $-1 \cdot 4$ \\
\hline $\mathrm{C}$ & 0.561432 & 0.453267 & -2.8243 \\
\hline $\mathrm{C}$ & 1.323351 & 1.069999 & -3.8 \\
\hline $\mathrm{C}$ & -0.561432 & -0.453267 & -2.824343 \\
\hline $\mathrm{C}$ & -0.890380 & -0.710249 & $-1 \cdot 4$ \\
\hline $\mathrm{C}$ & -2.709215 & -2.003602 & -2.230593 \\
\hline $\mathrm{C}$ & -1.323351 & -1.069999 & -3.80 \\
\hline $\mathrm{N}$ & 0.000000 & 0.000000 & -0.680329 \\
\hline $\mathrm{H}$ & 1.093347 & 0.941995 & -4.863444 \\
\hline $\mathrm{H}$ & -1.093347 & -0.941995 & -4.863444 \\
\hline $\mathrm{C}$ & -1.980538 & 1.498866 & 1.153 \\
\hline $\mathrm{C}$ & -1.980538 & -1.498866 & -1.153087 \\
\hline $\mathrm{C}$ & 1.980538 & -1.498866 & 1.153087 \\
\hline $\mathrm{C}$ & 1.980538 & 1.498866 & -1.153087 \\
\hline C & -4.895976 & -2.883571 & -3.095193 \\
\hline $\mathrm{C}$ & -4.895976 & 2.883571 & 3.095193 \\
\hline C & 4.895976 & -2.883571 & 3.095193 \\
\hline $\mathrm{C}$ & 4.895976 & 2.883571 & -3.09519 \\
\hline $\mathrm{C}$ & -9.194449 & 1.697002 & -0.737166 \\
\hline $\mathrm{C}$ & -9.194449 & -1.697002 & 0.73716 \\
\hline $\mathrm{C}$ & 9.194449 & -1.697002 & -0.73716 \\
\hline $\mathrm{C}$ & 9.194449 & 1.697002 & 0.73716 \\
\hline
\end{tabular}




\begin{tabular}{lrrr}
$\mathrm{N}$ & -2.371931 & -1.819771 & -3.517887 \\
$\mathrm{~N}$ & 2.371931 & -1.819771 & 3.517887 \\
$\mathrm{~N}$ & -2.371931 & 1.819771 & 3.517887 \\
$\mathrm{~N}$ & 2.371931 & 1.819771 & -3.517887 \\
$\mathrm{~N}$ & -4.423869 & -2.820032 & -0.754746 \\
$\mathrm{~N}$ & -4.423869 & 2.820032 & 0.754746 \\
$\mathrm{~N}$ & 4.423869 & -2.820032 & 0.754746 \\
$\mathrm{~N}$ & 4.423869 & 2.820032 & -0.754746 \\
$\mathrm{~N}$ & -9.400905 & -0.937381 & -1.512442 \\
$\mathrm{~N}$ & -9.400905 & 0.937381 & 1.512442 \\
$\mathrm{~N}$ & 9.400905 & -0.937381 & 1.512442 \\
$\mathrm{~N}$ & 9.400905 & 0.937381 & -1.512442 \\
$\mathrm{H}$ & 2.302924 & 1.680564 & -0.135765 \\
$\mathrm{H}$ & -2.302924 & -1.680564 & -0.135765 \\
$\mathrm{H}$ & -2.302924 & 1.680564 & 0.135765 \\
$\mathrm{H}$ & 2.302924 & -1.680564 & 0.135765 \\
$\mathrm{H}$ & 4.515434 & -2.821733 & 4.105994 \\
$\mathrm{H}$ & 9.441960 & -1.560261 & -1.782155 \\
$\mathrm{H}$ & 9.441960 & 1.560261 & 1.782155 \\
$\mathrm{H}$ & 4.515434 & 2.821733 & -4.105994 \\
$\mathrm{H}$ & -4.515434 & -2.821733 & -4.105994 \\
$\mathrm{H}$ & -9.441960 & -1.560261 & 1.782155 \\
$\mathrm{H}$ & -9.441960 & 1.560261 & -1.782155 \\
$\mathrm{H}$ & -4.515434 & 2.821733 & 4.105994 \\
$\mathrm{~N}$ & 5.697866 & -3.015247 & 0.483096 \\
$\mathrm{~N}$ & -5.697866 & -3.015247 & -0.483096 \\
$\mathrm{~N}$ & -5.697866 & 3.015247 & 0.483096 \\
$\mathrm{~N}$ & 5.697866 & 3.015247 & -0.483096 \\
$\mathrm{~N}$ & 8.613626 & -1.907804 & 1.925877 \\
$\mathrm{~N}$ & -8.613626 & -1.907804 & -1.925877 \\
$\mathrm{~N}$ & -8.613626 & 1.907804 & 1.925877 \\
$\mathrm{H}$ & -6.970761 & 3.192073 & 3.589696 \\
$\mathrm{H}$ & 6.970761 & -3.192073 & 3.589696 \\
$\mathrm{H}$ & 7.920039 & -3.413261 & -0.992778 \\
$\mathrm{H}$ & 7.920039 & 3.413261 & 0.992778 \\
$\mathrm{C}$ & 8.613626 & 1.907804 & -1.925877 \\
$\mathrm{C}$ & 6.222514 & -3.091651 & 2.814033 \\
$\mathrm{C}$ & -6.222514 & -3.091651 & -2.814033 \\
$\mathrm{C}$ & -6.222514 & 3.091651 & 2.814033 \\
$\mathrm{C}$ & 6.222514 & 3.091651 & -2.814033 \\
$\mathrm{C}$ & 8.361685 & -2.701499 & -0.307550 \\
$\mathrm{C}$ & -8.361685 & -2.701499 & 0.307550 \\
$\mathrm{C}$ & -8.361685 & 2.701499 & -0.307550 \\
$\mathrm{C}$ & 8.361685 & 2.701499 & 0.307550 \\
$\mathrm{H}$ & -6.970761 & -3.192073 & -3.589696 \\
$\mathrm{H}$ & -7.920039 & -3.413261 & 0.992778 \\
\hline
\end{tabular}

$\Delta \mathrm{H}=-3198.947154$

\section{NN- 6}

$\begin{array}{lllr}\text { C }-7.610469 & 4.240546 & 1.105701 \\ \text { C }-9.376728 & 4.049270 & -0.453846 \\ \text { C }-10.181183 & 3.787418 & 0.662377 \\ \text { C }-11.424394 & 2.984610 & 0.535921\end{array}$

\begin{tabular}{|c|c|c|c|}
\hline $\mathrm{C}$ & -6.187876 & 4.024993 & 1.469813 \\
\hline $\mathrm{C}$ & -4.527027 & 3.501286 & 3.066588 \\
\hline C & -3.797353 & 2.992694 & 1.984782 \\
\hline & -12.026259 & 2.398638 & 1.656005 \\
\hline $\mathrm{C}$ & -12.734836 & 0.727535 & 0.146922 \\
\hline & -9.790883 & 3.968565 & -1.450587 \\
\hline & -4.128102 & 3.416487 & 4.068442 \\
\hline & -11.843093 & 2.801442 & 2.643747 \\
\hline $\mathrm{C}$ & 7.610469 & -4.240546 & 1.105701 \\
\hline & 9.376728 & -4.049270 & -0.45384 \\
\hline & 10.181183 & -3.787418 & 0.66 \\
\hline C & 11.424394 & -2.984610 & 0.53 \\
\hline C & 6.18 & -4.024 & 1.4 \\
\hline $\mathrm{C}$ & 4.527027 & -3.501286 & 3.06 \\
\hline C & 3.7 & -2.992 & 1.9 \\
\hline $\mathrm{C}$ & 12.026259 & -2.398638 & 1.6 \\
\hline C & 12.73 & -0.72 & 0.1 \\
\hline & 9.790 & -3.96 & -1.45 \\
\hline & 4.12 & -3.41 & 4.0 \\
\hline $\mathrm{H}$ & 11.843 & -2.801 & 2.6 \\
\hline C & 7.6 & 4.24 & -1.10 \\
\hline C & 9.3767 & 4.04 & 0.45 \\
\hline $\mathrm{C}$ & 10.18 & 3.78 & -0.66 \\
\hline $\mathrm{C}$ & 11. & 2.98 & -0.53 \\
\hline C & 6.18 & 4.02 & -1.4 \\
\hline $\mathrm{C}$ & 4. & & -3.0 \\
\hline $\mathrm{C}$ & $3.79^{\circ}$ & 2.99 & $-1 \cdot 9$ \\
\hline C & 12 . & & $-1 \cdot 6$ \\
\hline 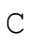 & 12.734 & 0.72 & -0.14 \\
\hline $\mathrm{H}$ & & 3.9 & 1.4 \\
\hline $\mathrm{H}$ & 4.128 & 3.41 & -4.06 \\
\hline $\mathrm{H}$ & 11. & 2.8 & -2.6 \\
\hline $\mathrm{C}$ & -7.610 & -4.240 & -1.10 \\
\hline $\mathrm{C}$ & -9 & -4.04 & 0.4 \\
\hline C & -10.18 & -3.787418 & -0.6 \\
\hline $\mathrm{C}$ & $-11 \cdot 4$ & -2.984610 & -0.5 \\
\hline $\mathrm{C}$ & -6.18 & -4.024993 & -1.46 \\
\hline $\mathrm{C}$ & -4.52 & -3.50 & -3.0 \\
\hline $\mathrm{C}$ & -3.79 & -2.992694 & -1.98 \\
\hline $\mathrm{C}$ & $-12 \cdot 0$ & -2.398638 & -1 \\
\hline$C$ & $-12 \cdot 7$ & -0.727535 & -0.1 \\
\hline $\mathrm{H}$ & -9.79 & -3.968565 & 1.4 \\
\hline & $-4.12 \varepsilon$ & -3.416487 & -4.06 \\
\hline $\mathrm{H}$ & -11.8 & -2.8 & -2 \\
\hline$C$ & 2.5728 & -2.177498 & 2.20 \\
\hline $\mathrm{C}$ & 1.88 & -1.60 & 1.1 \\
\hline C & 0.8536 & -0.752045 & $1.4^{7}$ \\
\hline $\mathrm{C}$ & 0.53 & -0.48 & 2.8 \\
\hline $\mathrm{C}$ & 1.2700 & -1.144033 & 3.7 \\
\hline $\mathrm{C}$ & -0.539 & 0.48 & 2.8 \\
\hline $\mathrm{C}$ & -0.8536 & 0.752045 & $1.4^{\circ}$ \\
\hline $\mathrm{C}$ & -1.8859 & 1.60 & 1.1 \\
\hline $\mathrm{C}$ & -2.572845 & 2.177498 & 2.2 \\
\hline $\mathrm{C}$ & -1.270069 & 1.144033 & 3.799199 \\
\hline 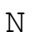 & 0.00 & 0.0 & 0.6 \\
\hline 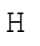 & 2.183 & -1.812991 & 0.1 \\
\hline & 1.060044 & -1.000512 & 4.8 \\
\hline
\end{tabular}




\begin{tabular}{|c|c|c|c|}
\hline & 2.183842 & 991 & 7659 \\
\hline & -1.060044 & 1.000512 & 4.855578 \\
\hline & 2.572845 & 2.177498 & -2.208879 \\
\hline & 1.885950 & 1.608885 & -1.138048 \\
\hline & 0.853644 & 0.752045 & -1.479535 \\
\hline & 0.539108 & 0.481191 & -2.820572 \\
\hline & 1.270069 & 1.144033 & -3.799199 \\
\hline & -0.539108 & -0.481191 & -2.820572 \\
\hline & -0 & -0 . & -1.479535 \\
\hline & -1.885950 & 885 & -1.138048 \\
\hline & -2.572845 & -2.177498 & -2.208879 \\
\hline & -1.270069 & -1.144033 & -3.799199 \\
\hline & 0.000000 & 0.000000 & -0.678732 \\
\hline & 2.183842 & 1.812991 & -0.117659 \\
\hline & 1.060044 & 1.000512 & -4.855578 \\
\hline & -2 & -1.812991 & -0.117659 \\
\hline & -1.060044 & -1.000512 & -4.855578 \\
\hline & 2.259571 & 1.967866 & -3.498622 \\
\hline & -2.259571 & -1.967866 & 8622 \\
\hline & -2.259571 & 1.967866 & 622 \\
\hline & 2.259571 & -1.967866 & 3.2 \\
\hline & -4.206402 & 3.133079 & 0.726287 \\
\hline & 4.206402 & -3.133079 & 0.726287 \\
\hline & 4.206402 & 3.133079 & -0.726287 \\
\hline & -4.206402 & -3.133079 & -0.726287 \\
\hline & -9.743458 & 3.973776 & 1.906076 \\
\hline & 9.743458 & -3.973776 & 1.906076 \\
\hline & 9.743458 & 3.973776 & -1.906076 \\
\hline & -9.743458 & -3.973776 & -1.906076 \\
\hline & -11.741471 & 2.611071 & $1-0.703748$ \\
\hline & 11.741471 & -2.61 & -0.7 \\
\hline & 11.741471 & 2.611071 & 0.703748 \\
\hline & -11.741471 & $1-2.611071$ & 0.703748 \\
\hline & 5.394694 & -3.642317 & 0.471485 \\
\hline & -5.394694 & 3.642317 & 0.471485 \\
\hline & 5.394694 & 3.642317 & -0.471485 \\
\hline & -5.394694 & -3.642317 & -0.471485 \\
\hline & 8.466743 & -4.206345 & 5832 \\
\hline & -8.466743 & 4.206345 & 2.125832 \\
\hline & 8.466743 & 4.206345 & -2.125832 \\
\hline & -8.466743 & -4.206345 & -2.125832 \\
\hline & 12.392763 & -1.483953 & -0.897027 \\
\hline & -12.392763 & 31.483953 & $3-0.897027$ \\
\hline & 12.392763 & 1.483953 & 0.897027 \\
\hline & -12.392763 & $3-1.483953$ & 0.897027 \\
\hline & 5.761950 & 4.034248 & -2.802283 \\
\hline & $=-5.761950$ & 4.034248 & 2.802283 \\
\hline & 5.761950 & -4.034248 & 2.802283 \\
\hline & -5.761950 & -4.034248 & -2.802283 \\
\hline & 8.042813 & 4.276001 & 0.224376 \\
\hline & -8.042813 & 4.276001 & -0.224376 \\
\hline & 8.042813 & -4.276001 & -0.224376 \\
\hline & -8.042813 & -4.276001 & 0.224376 \\
\hline & $=12.708715$ & 1.224278 & -1.454525 \\
\hline & $-12 \cdot 708$ & $5 \quad 1.2242^{\prime}$ & 81.454525 \\
\hline & 12.708715 & -1.224278 & 1.454525 \\
\hline & 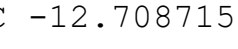 & 1.26 & \\
\hline
\end{tabular}

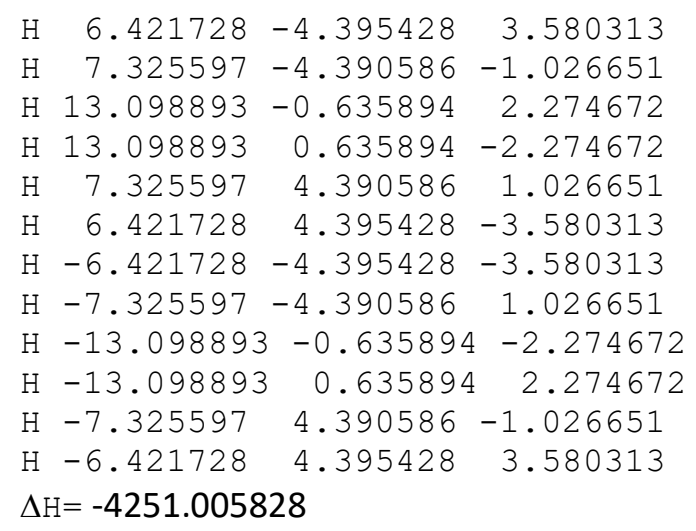

$8 N N-8$ 


\begin{tabular}{|c|c|c|c|}
\hline C & -0.840519 & -0.765274 & -1.477988 \\
\hline C & -2.521235 & -2.237057 & -2.198751 \\
\hline & -1.256018 & -1.165578 & -3.795025 \\
\hline & 0.000000 & 0.000000 & -0.678224 \\
\hline & 1.056832 & 1.014860 & -4.852478 \\
\hline & -1.056832 & -1.014860 & -4.852478 \\
\hline & -14.837710 & -3.161893 & $3-0.102309$ \\
\hline & -15.895288 & $3-0.742287$ & 22 \\
\hline & 15.895288 & 0.742287 & 0.021022 \\
\hline & 14.837710 & 3.161893 & -0.102309 \\
\hline & -14.837710 & 3.161893 & 0.102309 \\
\hline & 14.837710 & -3.161893 & 0.102309 \\
\hline & -15.895288 & $\begin{array}{ll}3 \quad 0.742287\end{array}$ & -0.0 \\
\hline & 15.895288 & -0.742287 & -0.021022 \\
\hline & 1.849372 & -1.646318 & 1.131460 \\
\hline & 1.849372 & 1.646318 & -1.131460 \\
\hline & -1.849372 & 1.646318 & 1.1 \\
\hline & -1.849372 & -1.646318 & -1.131460 \\
\hline & 2.222667 & -2.015294 & 3.4 \\
\hline & -2.222667 & 2.015294 & 3.490001 \\
\hline & 2.22 & 2.015 & -3.4 \\
\hline & -2.222667 & -2.015294 & -3.490001 \\
\hline & 4.08 & -3.274 & 0.7 \\
\hline & -4.089552 & 3.274153 & 0.702688 \\
\hline & 4.08 & 3.274 & -0.7 \\
\hline & -4.08 & -3.27 & -0.7 \\
\hline & 9.37 & -5.445 & 1.8 \\
\hline & -9.37 & 5.44 & 1.8 \\
\hline & 9.37 & 5.44 & $-1 \cdot 8$ \\
\hline & -9.37 & -5.44 & $-1 \cdot 8$ \\
\hline & 4.36 & -3.717 & 3.036 \\
\hline $\mathrm{C}$ & 4.36 & 3.71 & $-3 \cdot 0$ \\
\hline C & -4.369131 & 3.717225 & 3.036 \\
\hline C & -4.36 & -3.71 & -3.0 \\
\hline C & 9.056073 & -5.164117 & -0.512535 \\
\hline C & 9.05 & 5.16 & 0. \\
\hline C & -9.056073 & 5.164117 & -0.512535 \\
\hline $\mathrm{C}$ & -9.056073 & -5.164117 & 0.5 \\
\hline $\mathrm{H}$ & 2.134116 & -1.861162 & 0.1 \\
\hline $\mathrm{H}$ & -2.134116 & 1.861 & 0 . \\
\hline $\mathrm{H}$ & -2.134116 & -1.861162 & -0.109589 \\
\hline $\mathrm{H}$ & 2.134116 & 1.861162 & -0.109589 \\
\hline $\mathrm{N}$ & 11.839442 & -4.943439 & -0.676133 \\
\hline $\mathrm{N}$ & -11.839442 & 4.943439 & -0.676133 \\
\hline $\mathrm{N}$ & 11.839442 & 4.943439 & 0.676133 \\
\hline $\mathrm{N}$ & -11.839442 & $2-4.943439$ & 0.676133 \\
\hline $\mathrm{N}$ & 15.752245 & -1.344536 & 1.158460 \\
\hline $\mathrm{N}$ & -15.752245 & $5-1.344536$ & $5-1.158460$ \\
\hline דג & 15.752245 & 1.344536 & -1.158460 \\
\hline $2 v$ & -15.752245 & 1.344536 & 1.158460 \\
\hline C & 12.141955 & -5.168641 & 1.684172 \\
\hline $\mathrm{C}$ & 12.141955 & 5.168641 & -1.684172 \\
\hline $\mathrm{C}$ & -12.141955 & 5.168641 & 1.684172 \\
\hline $\mathrm{C}$ & -12.141955 & $5-5.168641$ & -1.684172 \\
\hline $\mathrm{C}$ & 15. & -1.43 & -1.228614 \\
\hline $\mathrm{C}$ & 15.750527 & 1.435827 & 1.228614 \\
\hline & -15.750527 & $7-1.435827$ & 1.228614 \\
\hline
\end{tabular}

\begin{tabular}{|c|c|c|c|}
\hline & -15.750527 & 1.435827 & -1.228614 \\
\hline & 9.497759 & 5.136919 & 1.500199 \\
\hline & 11.727853 & 5.472443 & -2.636746 \\
\hline & 15.968717 & 0.942951 & 2.167100 \\
\hline & 15.968717 & -0.942951 & -2.167100 \\
\hline & 11.727853 & -5.472443 & 2.636746 \\
\hline & 9.497759 & -5.136919 & -1.500199 \\
\hline & -3.986854 & 3.599223 & 4.041057 \\
\hline & -9.497759 & 5.136919 & $-1 \cdot 5$ \\
\hline & 11.727853 & 5.472443 & 2.63 \\
\hline & 15.968717 & 0.942951 & -2 \\
\hline & 15.968717 & $7-0.942951$ & 00 \\
\hline & 11.7 & $3-5.472443$ & -2 \\
\hline & 9.497 & -5.136919 & 1.5 \\
\hline & -3 & -3.5 & -4.0 \\
\hline & 4 & -3.599 & 4.04 \\
\hline & & 3.5 & -4.0 \\
\hline & 5.20 & -3.920 & 0.43 \\
\hline & 5. & 3.92 & -0.4 \\
\hline & -5 & 3.92 & 0 . \\
\hline & -5 & -3.92 & -0 . \\
\hline & 8 . & -5.247952 & 2.05 \\
\hline & & 5.2 & -2 . \\
\hline & -8 & 5.24 & 2 \\
\hline & -8 & -5.24 & -2.0 \\
\hline & & -4.4 & -0 . \\
\hline & 13. & 4.430 & 0.7 \\
\hline & -1 & 4.43 & -0 \\
\hline & -13 & $3-4.43$ & 80 \\
\hline & & -2.5 & \\
\hline N & 15.22 & 2.55 & -1.21 \\
\hline & -1 & -2.5 & $5-1$ \\
\hline 1 & -15.2 & 2.550 & 1. \\
\hline C & & -4.3 & \\
\hline C & 5.527 & 4.39 & -2.75 \\
\hline $\mathrm{C}$ & -5.52 & 4.3 & 2.7 \\
\hline o & -5.527 & -4.39 & -2.75 \\
\hline C & $7.71\}$ & -4.94 & -0.2 \\
\hline C & 7.718 & 4.94 & 0.2 \\
\hline $\mathrm{C}$ & -7.718 & 4.94 & -0.2 \\
\hline c & -7.718 & -4.94 & 0.2 \\
\hline C & 13.40 & -4.63 & 1.5 \\
\hline C & 13.400 & 4.63 & $-1 \cdot 5$ \\
\hline C & -13.4 & 4.6 & 66 \\
\hline $\mathrm{C}$ & -13.40 & -4.63 & $9-1$. \\
\hline C & 15.19 & -2.69 & -1.1 \\
\hline $\mathrm{C}$ & 15.197 & 2.69 & 1.1 \\
\hline C & -15.1 & $3-2.6$ & 17 \\
\hline $\mathrm{C}$ & -15.197 & $3 \quad 2.690508$ & B -1 . \\
\hline $\mathrm{H}$ & 6.140 & -4.850 & 3.5 \\
\hline $\mathrm{H}$ & 7.019772 & -4.739144 & $-1 \cdot 0$ \\
\hline $\mathrm{H}$ & 14.0527 & -4.47 & 2.4 \\
\hline $\mathrm{H}$ & 14.9480 & -3.260333 & -2.0 \\
\hline $\mathrm{H}$ & 14. & 3.260333 & 2.0 \\
\hline $\mathrm{H}$ & 14.052740 & 4.479848 & -2.4 \\
\hline $\mathrm{H}$ & 7. & 4.73 & 1. \\
\hline & 6.140202 & 4.850 & -3 \\
\hline
\end{tabular}


$\begin{array}{cccc}H & -6.140202 & -4.850529 & -3.522408 \\ H & -7.019772 & -4.739144 & 1.091743 \\ H & -14.052740 & -4.479848 & -2.408532 \\ H & -14.948050 & -3.260333 & 2.050299 \\ H & -14.948050 & 3.260333 & -2.050299 \\ H & -14.052740 & 4.479848 & 2.408532 \\ H & -7.019772 & 4.739144 & -1.091743 \\ H & -6.140202 & 4.850529 & 3.522408\end{array}$ $\Delta \mathrm{H}=-5303.054623$

\section{$8 C C-4$}

\begin{tabular}{|c|c|c|c|}
\hline C & -8.419188 & 2.307056 & 1.447081 \\
\hline 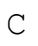 & -10.132642 & 0.667746 & 0.327756 \\
\hline 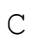 & -7.022208 & 2.501905 & 1.902201 \\
\hline $\mathrm{C}$ & -4.468937 & 1.933612 & 2.338815 \\
\hline C & 8.419188 & -2.307056 & 1.447081 \\
\hline $\mathrm{C}$ & 10.132642 & -0.667746 & 0.327756 \\
\hline C & 7.022208 & -2.501905 & 1.902201 \\
\hline 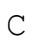 & 4.468937 & -1.933612 & 2.338815 \\
\hline C & 8.419188 & 2.307056 & -1.447081 \\
\hline C & 10.132642 & 0.667746 & -0.32 \\
\hline C & 7.022208 & 2.501905 & -1.9 \\
\hline $\mathrm{C}$ & 4.468937 & 1.933612 & -2.33 \\
\hline C & -8.419188 & $-2 \cdot 30$ & -1.4 \\
\hline$C$ & -10.1 & -0 & -0 . \\
\hline C & -7.022208 & -2.50 & $-1 \cdot 9$ \\
\hline C & -4.468937 & -1.933612 & -2.338815 \\
\hline C & 3.09 & -1.372229 & 2.47 \\
\hline $\mathrm{C}$ & 1.123881 & -0.370182 & 1.583956 \\
\hline C & 0.676386 & -0.263431 & 2.91 \\
\hline C & 1.478256 & -0.701411 & 3.949238 \\
\hline C & -0.676386 & 0.263431 & 2.91 \\
\hline $\mathrm{C}$ & -1.123881 & 0.370182 & 1.583956 \\
\hline $\mathrm{C}$ & -3.098390 & 1.372229 & 2.476051 \\
\hline $\mathrm{C}$ & -1.478256 & 0.701411 & 3.949238 \\
\hline $\mathrm{H}$ & 1.153465 & -0.639723 & 4.984375 \\
\hline $\mathrm{H}$ & -1.153465 & 0.639723 & 4.984375 \\
\hline C & 3.098390 & 1.372229 & -2.476051 \\
\hline $\mathrm{C}$ & 1.123881 & 0.370182 & -1.583956 \\
\hline $\mathrm{C}$ & 0.676386 & 0.263431 & -2.911468 \\
\hline $\mathrm{C}$ & 1.478256 & 0.701411 & -3.949238 \\
\hline $\mathrm{C}$ & -0.676386 & -0.263431 & -2.911468 \\
\hline $\mathrm{C}$ & -1.123881 & -0.370182 & -1.583956 \\
\hline $\mathrm{C}$ & -3.098390 & -1.372229 & -2.476051 \\
\hline $\mathrm{C}$ & -1.478256 & -0.701411 & -3.949238 \\
\hline $\mathrm{H}$ & 1.153465 & 0.639723 & -4.984375 \\
\hline $\mathrm{H}$ & -1.153465 & -0.639723 & -4.984375 \\
\hline $\mathrm{C}$ & -2.357439 & 0.967172 & 1.356115 \\
\hline $\mathrm{C}$ & -2.357439 & -0.967172 & -1.356115 \\
\hline $\mathrm{C}$ & 2.357439 & -0.967172 & 1.356115 \\
\hline $\mathrm{C}$ & 2.357439 & 0.967172 & -1.356115 \\
\hline $\mathrm{C}$ & -5.308105 & -2.043214 & -3.456464 \\
\hline $\mathrm{C}$ & -5.308105 & 2.043214 & 6464 \\
\hline $\mathrm{C}$ & 5.308105 & -2.043214 & 3.456464 \\
\hline & 5.308 & 3214 & -3. \\
\hline
\end{tabular}

\begin{tabular}{|c|c|c|c|}
\hline C & -9.754302 & 1.787620 & -0.423759 \\
\hline C & -9.754302 & -1.787620 & 0.423759 \\
\hline c & 9.754302 & -1.787620 & -0.423759 \\
\hline C & 9.754302 & 1.787620 & 0.423759 \\
\hline $\mathrm{N}$ & -2.673571 & -1.232726 & -3.735100 \\
\hline $\mathrm{N}$ & 2.673571 & -1.232726 & 3.735100 \\
\hline $\mathrm{N}$ & -2.673571 & 1.232726 & 3.735100 \\
\hline $\mathrm{N}$ & 2.673571 & 1.232726 & -3.73 \\
\hline $\mathrm{N}$ & -4.875105 & -2.258701 & -1.114965 \\
\hline $\mathrm{N}$ & -4.875105 & 2.258701 & 4965 \\
\hline $\mathrm{N}$ & 4.875105 & -2.258701 & 1.11 \\
\hline $\mathrm{N}$ & 4.875105 & 2.258701 & -1.1 \\
\hline $\mathrm{N}$ & -9.981367 & -0.647033 & -1.65 \\
\hline $\mathrm{N}$ & -9.981367 & 0.647033 & 1.655067 \\
\hline $\mathrm{N}$ & 9.981367 & -0.647 & 1.6 \\
\hline $\mathrm{N}$ & 9.981367 & 0.647033 & -1.655067 \\
\hline $\mathrm{H}$ & 2.76 & 1.1 & -0.3 \\
\hline $\mathrm{H}$ & -2.764408 & $-1 \cdot 13$ & -0.3 \\
\hline $\mathrm{H}$ & -2.764408 & 1.1 & 0.367955 \\
\hline $\mathrm{H}$ & 2.764408 & -1.13 & 0.3 \\
\hline $\mathrm{H}$ & 4.910558 & -1.84 & 4.44 \\
\hline & 10.02 & -1.8 & $-1 \cdot 4$ \\
\hline $\mathrm{H}$ & 10.022991 & 1.86 & $1.469^{\circ}$ \\
\hline $\mathrm{H}$ & 4.91 & 1.8 & -4.4 \\
\hline $\mathrm{H}$ & -4.910558 & -1.845948 & -4.443050 \\
\hline F & -10.0 & -1.8 & 1.4 \\
\hline $\mathrm{H}$ & -10.022991 & 1.861493 & $3-1.469791$ \\
\hline H & -4.91 & 1.84 & 4.44 \\
\hline$\Lambda$ & 6.145373 & -2.535505 & 0.898385 \\
\hline$\Lambda$ & -6.145373 & -2.53 & -0.8 \\
\hline $\mathrm{N}$ & -6.145373 & 2.535505 & 0.898385 \\
\hline $\mathrm{N}$ & 6.14 & 2.53 & -0.8 \\
\hline $\mathrm{N}$ & 9.123943 & -1.472318 & 2.217444 \\
\hline $\mathrm{N}$ & -9.12 & -1.4 & -2.21 \\
\hline $\mathrm{N}$ & -9.123943 & 1.47 & 2.217444 \\
\hline $\mathrm{N}$ & 9.12 & $1.4^{\circ}$ & -2.21 \\
\hline $\mathrm{C}$ & $6.62 \varepsilon$ & -2.334808 & 3.233374 \\
\hline $\mathrm{C}$ & -6.62 & $-2 \cdot 3$ & -3.233374 \\
\hline $\mathrm{C}$ & -6.628916 & 2.33 & 3.233374 \\
\hline $\mathrm{C}$ & 6.62 & 2.3 & -3.23 \\
\hline $\mathrm{C}$ & 8.843020 & -2.635635 & 0.157453 \\
\hline $\mathrm{C}$ & -8.843 & -2.6 & -0.15 \\
\hline $\mathrm{C}$ & -8.843020 & 2.635635 & 0.157453 \\
\hline $\mathrm{C}$ & 8.84 & 2.6 & -0.157453 \\
\hline . & -7.361319 & -2.361248 & -4.030055 \\
\hline $\mathrm{H}$ & -8.361922 & -3.4 & 0.399879 \\
\hline $\mathrm{H}$ & -8.361922 & 3.428846 & -0.399879 \\
\hline $\mathrm{H}$ & -7.361319 & 1248 & 4.030055 \\
\hline $\mathrm{H}$ & 7.361319 & -2.361248 & 4.030055 \\
\hline $\mathrm{H}$ & 8.361922 & -3.428846 & -0.399879 \\
\hline $\mathrm{H}$ & 8.361922 & 3.428846 & 0.399879 \\
\hline $\mathrm{H}$ & 7.361319 & 2.361248 & -4.030055 \\
\hline $\mathrm{C}$ & 0.000 & 000 & 42 \\
\hline $\mathrm{C}$ & 0.000000 & 0.000 & -0.68034 \\
\hline
\end{tabular}

$\Delta \mathrm{H}=-3165.637872$ 


\section{$8 C C-6$}

C -8.200892

C -9.974290

C -10.807963

3.507015

3. 698288

3.414020

1.556050

C -12.137876

2.785741

C -6.798848

C -5.140989

C -4.395667

3.150819

2.456591

2.093447

2.121043

0.704349

C -13.613765

3.781730

$\mathrm{H}-10.392215$

$\mathrm{H}-4.735207$

2.280365

2.378779

C $8.200892-3.507015$

C $9.974290-3.698288$

C $10.807963-3.414020$

C $12.137876-2.785741$

C $6.798848-3.150819$

C $5.140989-2.456591$

C $4.395667-2.093447$

C $12.793114-2.121043$

C $13.613765-0.704349$

H $10.392215-3.781730$

$\mathrm{H} \quad 4.735207 \quad-2.280365$

H 12.577295

C 8.200892

C 9.974290

C 10.807963

C 12.137876

C 6.798848

C 5.140989

C 4.395667

C 12.793114

C 13.613765

H 10.392215

$\mathrm{H} \quad 4.735207$

H 12.577295

C $-8.200892-3.507015-1.556050$

C $-9.974290-3.698288-0.004193$

C $-10.807963-3.414020-1.093594$

C $-12.137876-2.785741-0.888844$

C $-6.798848-3.150819-1.887395$

C $-5.140989-2.456591-3.420033$

C $-4.395667-2.093447-2.290455$

C $-12.793114-2.121043-1.932178$

C $-13.613765-0.704349-0.234675$

$\mathrm{H}-10.392215-3.781730 \quad 0.990717$

$\mathrm{H}-4.735207-2.280365-4.406968$

$\mathrm{H}-12.577295-2.378779-2.961024$

$\begin{array}{llll}\text { C } 3.079698 & -1.415468 & 2.443812\end{array}$

C $2.344434 \quad-0.980454 \quad 1.334577$

C $1.121994-0.368996 \quad 1.577465$

$\begin{array}{llll}\text { C } & 0.678066 & -0.262872 & 2.906196\end{array}$

$\begin{array}{llll}\text { C } & 1.487700 & -0.707715 & 3.935681\end{array}$

$\begin{array}{llll}\text { C }-0.678066 & 0.262872 & 2.906196\end{array}$

C $-1.121994 \quad 0.368996 \quad 1.577465$

\begin{tabular}{|c|c|c|c|}
\hline C & -2.344434 & 0.980454 & 1.334577 \\
\hline $\mathrm{C}$ & -3.079698 & 1.415468 & 2.443812 \\
\hline C & -1.487700 & 0.707715 & 3.935681 \\
\hline & 2.737243 & -1.156857 & 341559 \\
\hline $\mathrm{H}$ & 1.177848 & -0.639035 & 4.974954 \\
\hline & -2.737243 & 1.156857 & 0.341559 \\
\hline & -1.177848 & 0.639035 & 4.974954 \\
\hline & 3.079698 & 1.415468 & -2.443812 \\
\hline & 2.344434 & 0.980454 & $-1 \cdot 3$ \\
\hline & 1.121994 & 0.368996 & -1.5 \\
\hline & 0.678066 & 0.262872 & -2 . \\
\hline $\mathrm{C}$ & 1.487700 & 0.707715 & -3 \\
\hline & -0.678066 & -0.26 & -2 \\
\hline & -1.121 & -0.36 & -1.5 \\
\hline $\mathrm{C}$ & -2.34 & -0.98 & $-1 \cdot 3$ \\
\hline $\mathrm{C}$ & -3.07 & -1.41 & $-2 \cdot 4$ \\
\hline & -1.48 & -0.70 & -3 \\
\hline & 2.737 & 1.15 & -0.3 \\
\hline & 1.17 & 0.6 & -4 \\
\hline & -2.73 & -1.15 & -0. \\
\hline & -1.17 & -0.6 & -4 \\
\hline & 2.668 & 1.26 & -3.7 \\
\hline & -2.66 & -1.26 & -3 \\
\hline $\mathrm{N}$ & -2 . & 1.2 & 917 \\
\hline & 2.66 & -1.26 & 3. \\
\hline $\mathrm{N}$ & -4 & 2.3 & \\
\hline $\mathrm{N}$ & 4.822 & -2.326545 & 1. \\
\hline $\mathrm{N}$ & 4. & & -1 \\
\hline $\mathrm{N}$ & -4.822 & -2.326545 & -1.052702 \\
\hline $\mathrm{N}$ & -10 . & 3. & 579 \\
\hline N & 10.35 & -3.396337 & 2.3 \\
\hline 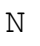 & 10. & 3. & $-2 \cdot 3$ \\
\hline N & -10.352 & -3.396337 & -2.344579 \\
\hline N & -12 & 2 . & -0 . \\
\hline $\mathrm{N}$ & 12.49 & -2.611060 & -0.383068 \\
\hline N & 12.499 & 2.6 & 0.3 \\
\hline $\mathrm{N}$ & -12.49 & -2.611060 & 3068 \\
\hline $\mathrm{N}$ & 6.017 & -2.847878 & 0.85 \\
\hline $\mathrm{N}$ & -6.017871 & 2.847878 & 0.85 \\
\hline $\mathrm{N}$ & 6.017 & 2.84 & -0.85 \\
\hline 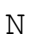 & -6.017871 & -2.847878 & -0.85 \\
\hline $\mathrm{N}$ & 9.057 & -3.4 & 2.57 \\
\hline זר & -9.057985 & 3.453411 & 2.574636 \\
\hline $\mathrm{N}$ & 9.057 & 3.4 & -2.57 \\
\hline $\mathrm{N}$ & -9.057985 & -3.453411 & -2.574636 \\
\hline $\mathrm{N}$ & 13.232 & -1.56 & $-0.7 C$ \\
\hline ד & -13.232374 & 1.568155 & -0.709042 \\
\hline $\mathrm{N}$ & 13.2323 & 1.5 & 0.709042 \\
\hline $\mathrm{N}$ & -13.232374 & -1.568155 & 0.709042 \\
\hline $\mathrm{C}$ & 6.380085 & 037 & -3.214036 \\
\hline $\mathrm{C}$ & -6.380085 & 3.002037 & 3.214036 \\
\hline $\mathrm{C}$ & 6.380085 & -3.0 & 3.21 \\
\hline $\mathrm{C}$ & -6.380085 & -3.0 & -3.214036 \\
\hline $\mathrm{C}$ & 8.623268 & 3.735298 & -0.242237 \\
\hline $\mathrm{C}$ & -8.623268 & 3.735298 & 0.242237 \\
\hline $\mathrm{C}$ & 8.623268 & -3.73 & 0.242237 \\
\hline 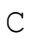 & -8.623268 & -3.735298 & -0.242237 \\
\hline
\end{tabular}




$\begin{array}{lcrr}\text { C } & 13.566255 & 1.037657 & -1.592437 \\ \text { C } & -13.566255 & 1.037657 & 1.592437 \\ \text { C } & 13.566255 & -1.037657 & 1.592437 \\ \text { C } & -13.566255 & -1.037657 & -1.592437 \\ \text { H } & 7.041517 & -3.277826 & 4.024811 \\ \text { H } & 7.894947 & -3.855083 & -0.549291 \\ \text { H } & 13.998430 & -0.383087 & 2.338014 \\ \text { H } & 13.998430 & 0.383087 & -2.338014 \\ \text { H } & 7.894947 & 3.855083 & 0.549291 \\ \text { H } & 7.041517 & 3.277826 & -4.024811 \\ \text { H } & -7.041517 & -3.277826 & -4.024811 \\ \text { H } & -7.894947 & -3.855083 & 0.549291 \\ \text { H } & -13.998430 & -0.383087 & -2.338014 \\ \text { H } & -13.998430 & 0.383087 & 2.338014 \\ \text { H } & -7.894947 & 3.855083 & -0.549291 \\ \text { H } & -7.041517 & 3.277826 & 4.024811 \\ \text { C } & 0.000000 & 0.000000 & 0.678904 \\ \text { C } & 0.000000 & 0.000000 & -0.678904\end{array}$

$\Delta \mathrm{H}=-4217.692865$

\section{$8 C C-8$}

$\begin{array}{lccc}\text { C } & -8.045447 & 3.867641 & 1.474292 \\ \text { C } & -10.584492 & 4.541756 & 1.086074 \\ \text { C } & -12.065537 & 4.603993 & 0.996870 \\ \text { C } & -6.716173 & 3.301147 & 1.816662 \\ \text { C } & -4.395133 & 2.086025 & 2.260021 \\ \text { C } & -14.606511 & 3.934864 & 0.673466 \\ \text { C } & 8.045447 & -3.867641 & 1.474292 \\ \text { C } & 10.584492 & -4.541756 & 1.086074 \\ \text { C } & 12.065537 & -4.603993 & 0.996870 \\ \text { C } & 6.716173 & -3.301147 & 1.816662 \\ \text { C } & 4.395133 & -2.086025 & 2.260021 \\ \text { C } & 14.606511 & -3.934864 & 0.673466 \\ \text { C } & 8.045447 & 3.867641 & -1.474292 \\ \text { C } & 10.584492 & 4.541756 & -1.086074 \\ \text { C } & 12.065537 & 4.603993 & -0.996870 \\ \text { C } & 6.716173 & 3.301147 & -1.816662 \\ \text { C } & 4.395133 & 2.086025 & -2.260021 \\ \text { C } & 14.606511 & 3.934864 & -0.673466 \\ \text { C } & -8.045447 & -3.867641 & -1.474292 \\ \text { C } & -10.584492 & -4.541756 & -1.086074 \\ \text { C } & -12.065537 & -4.603993 & -0.996870 \\ \text { C } & -6.716173 & -3.301147 & -1.816662 \\ \text { C } & -4.395133 & -2.086025 & -2.260021 \\ \text { C } & -14.606511 & -3.934864 & -0.673466 \\ \text { C } & 3.092870 & -1.387063 & 2.431172 \\ \text { C } & 1.123527 & -0.358077 & 1.576077 \\ \text { C } & 0.682518 & -0.253849 & 2.906062 \\ \text { C } & 1.506134 & -0.684157 & 3.931572 \\ \text { C } & -0.682518 & 0.253849 & 2.906062 \\ \text { C } & -1.123527 & 0.358077 & 1.576077 \\ \text { C } & -3.092870 & 1.387063 & 2.431172 \\ \text { C } & -1.506134 & 0.684157 & 3.931572 \\ \text { H } & 1.204411 & -0.612786 & 4.973034 \\ \text { H } & -1.1004411 & 0.612786 & 4.973034\end{array}$

\begin{tabular}{|c|c|c|c|}
\hline $\mathrm{C}$ & 3.092870 & 1.387063 & -2.431172 \\
\hline C & 1.123527 & 0.358077 & -1.576077 \\
\hline C & 0.682518 & 0.253849 & -2.906062 \\
\hline & 1.506134 & 0.684157 & 1572 \\
\hline & -0.682518 & -0.253849 & -2.906062 \\
\hline & -1.123 & -0.358077 & -1.576077 \\
\hline & -3.0 & -1.387063 & -2.431172 \\
\hline & -1.506134 & -0.684157 & -3.9 \\
\hline & 1.20 & 0.612786 & -4.9 \\
\hline & -1.204411 & -0.612 & -4.9 \\
\hline & -15 & -3.062723 & $3-0$. \\
\hline & -16.982237 & -0.74 & $3-0$. \\
\hline & 16. & 0.74 & -0.0 \\
\hline & 15.77 & 3.06 & -0.4 \\
\hline & -15.7 & 3.0 & 0 \\
\hline & 15.7 & -3.06 & 0.4 \\
\hline & -16 & 0.7 & \\
\hline & 16.9 & -0.74 & 0.0 \\
\hline & 2.34 & -0.9 & 1.3 \\
\hline & 2.347 & 0.9 & $-1 \cdot 32$ \\
\hline & -2.34 & 0.9 & 1.3 \\
\hline & -2.34 & -0.96 & -1.32 \\
\hline & 2.6 & $-1 \cdot 21$ & 3. \\
\hline & -2 . & 1.2 & 5 \\
\hline & 2.6 & 1.21 & -3 \\
\hline & -2 . & -1.2 & -3 \\
\hline & 4.8 & -2.2 & 63 \\
\hline 1 & -4 . & & 3 \\
\hline 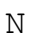 & 4.81 & 2.2 & $-1 \cdot 0$ \\
\hline 1 & -4 & -2.29 & $-1 \cdot 0$ \\
\hline & 10. & -4.490 & 769 \\
\hline $\mathrm{N}$ & -10 & 4.49 & 69 \\
\hline & 10 . & 74 & $-2 \cdot 31$ \\
\hline $\mathrm{N}$ & -10 & -4.5 & -2 . \\
\hline C & 5.126014 & -2.51 & 623 \\
\hline C & 5 . & 2.51 & $-3 \cdot 3$ \\
\hline & -5.126014 & 2.51 & 5623 \\
\hline $\mathrm{C}$ & -5.12 & -2.51 & -3.3 \\
\hline C & 9.78 & -4.36 & -0.0 \\
\hline C & 9.7 & 4.3 & 521 \\
\hline C & -9.786 & 4.36 & -0.05 \\
\hline C & -9.7 & -4.3 & 0521 \\
\hline & 2.733 & -1.14 & 2234 \\
\hline $\mathrm{H}$ & -2.73 & 1.1 & 2234 \\
\hline & -2.73 & $-1 \cdot 1$ & -0.3 \\
\hline $\mathrm{H}$ & 2.7 & 1.1 & -0.3 \\
\hline & 12.57 & -4.513080 & -0.228485 \\
\hline & -12.5 & 3080 & -0.228485 \\
\hline $\mathrm{N}$ & 12.577508 & 4.513080 & 0.228485 \\
\hline 10 & -12.577508 & -4.513080 & 0.228485 \\
\hline $\mathrm{N}$ & 16.814617 & -1.221016 & 1.283185 \\
\hline 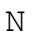 & -16.8 & -1.221016 & -1.283185 \\
\hline דה & 16.81 & 1.221016 & -1.283185 \\
\hline $\mathrm{N}$ & -16.8 & 1.221016 & 1.283185 \\
\hline$\Omega$ & 12.8 & -4.572472 & 3041 \\
\hline $\mathrm{C}$ & 12.86 & 2472 & -2.143041 \\
\hline & -12 & 472 & \\
\hline
\end{tabular}




\begin{tabular}{|c|c|c|c|}
\hline & 0 & -4 & \\
\hline & & & -1 \\
\hline & 814318 & 1.545368 & 1.082198 \\
\hline & 318 & -1.545368 & 198 \\
\hline & 6.814318 & 1.545368 & -1.082198 \\
\hline & 0.223469 & 4.453217 & 1.036522 \\
\hline & 12. & 4.736358 & -3 \\
\hline & 17.063788 & 1.162320 & 2.0 \\
\hline & 17.063788 & -1.162320 & -2.063 \\
\hline & 12.427594 & -4.736358 & 3.117 \\
\hline & 10.223469 & -4.453217 & -1.036522 \\
\hline & -4.738172 & 2.329928 & 4.370050 \\
\hline & -10.223469 & 4.453217 & -1.03652 \\
\hline & -12.427594 & 4.736358 & 3. \\
\hline & -17.063788 & 1.162320 & -2.063187 \\
\hline & -17.063788 & -1.162320 & 2.063187 \\
\hline & -12.427594 & -4.736358 & -3.117189 \\
\hline & 3469 & -4.453217 & 1.036522 \\
\hline & -4 & -2.329928 & -4.370050 \\
\hline & 4.73 & -2.329928 & 4.3 \\
\hline & 4.738172 & 2.329928 & -4.370050 \\
\hline & 5.966080 & -2.896575 & 0.795444 \\
\hline & 5.966080 & 2.896575 & -0.7 \\
\hline & -5 . & 575 & \\
\hline & -5.9 & -2.896575 & 5444 \\
\hline & 8.827285 & -4.16 & 2.5 \\
\hline & 8.827285 & 4.162632 & -2.510571 \\
\hline & -8.827285 & 4.162632 & 2.510571 \\
\hline & -8.827285 & -4.162632 & -2.510571 \\
\hline & 13. & $-4 \cdot 1$ & -0.3 \\
\hline & 13.840272 & 4.176955 & 0.388739 \\
\hline & -13.840272 & $2 \quad 4.176955$ & -0.38873 \\
\hline & -13.840272 & $2-4.176955$ & 0.388739 \\
\hline & 16.215624 & -2.379842 & 1.457155 \\
\hline & 16.215624 & 2.379842 & -1.457155 \\
\hline & -16 & $4-2.379842$ & -1.457155 \\
\hline & -16.215624 & $\begin{array}{l}4.379842 \\
\end{array}$ & 1.457155 \\
\hline & 6.321581 & -3.138924 & 3.149553 \\
\hline & 6.321581 & 3.138924 & -3.149553 \\
\hline & -6.321581 & 3.138924 & 3.14955 \\
\hline & -6.321581 & -3.138924 & -3.149553 \\
\hline & 8.47 & -4.011307 & 0.150220 \\
\hline & 8.47 & 4.01 & -0.150220 \\
\hline & -8.475035 & 4.011307 & 0.150220 \\
\hline & -8.475035 & -4.011307 & -0.150220 \\
\hline & 14.186710 & -4.226925 & 1.976078 \\
\hline & 14.186710 & 4.226925 & -1.976078 \\
\hline & -14.186710 & 4.226925 & $5 \quad 1.976078$ \\
\hline & -14.186710 & -4.226925 & $5-1.976078$ \\
\hline & 16.182220 & -2.750429 & -0.901330 \\
\hline & 16.182220 & 2.750429 & 0.901330 \\
\hline & -16.182220 & -2.750429 & 0.901330 \\
\hline & -16.182220 & 2.750429 & -0.901330 \\
\hline & 6.962168 & -3.489434 & 3.948025 \\
\hline & 7.791705 & -3.803974 & -0.663250 \\
\hline & 861575 & -4.088195 & 2.81063 \\
\hline & & -3.3 & \\
\hline
\end{tabular}

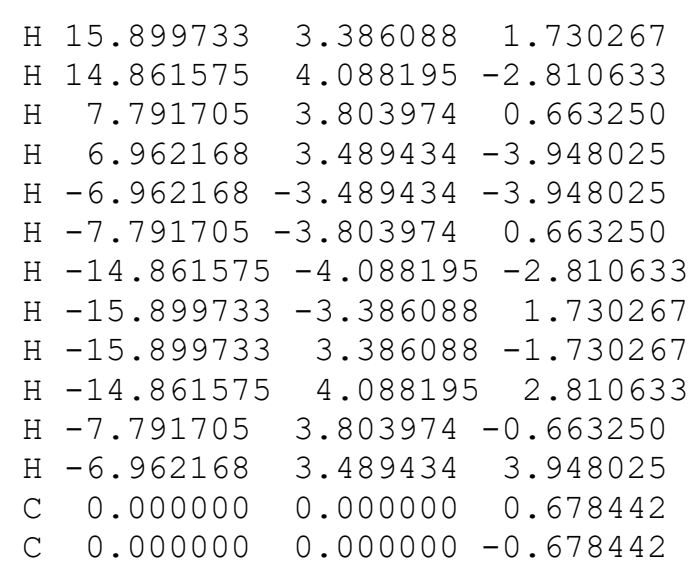

$\Delta \mathrm{H}=-5269.741098$

\section{NN-5}




\begin{tabular}{|c|c|c|c|}
\hline & 95 & -6 & -3 \\
\hline & -1.203118 & -11.296434 & -1.963437 \\
\hline & 1.961969 & 2.676612 & 2.175217 \\
\hline & 0.693658 & 0.901671 & 1.660232 \\
\hline & 0.443926 & 0.567427 & 3.005364 \\
\hline & 1.060610 & 1.348183 & 3.972174 \\
\hline & 1.833294 & 2.414387 & 3.545283 \\
\hline & -0.443926 & -0.567427 & 3.005364 \\
\hline & -0.693658 & -0.901671 & \\
\hline & -1.961969 & -2.676612 & 2.175217 \\
\hline & -1.833294 & -2.414387 & 3.545283 \\
\hline & -1.060610 & -1.348183 & 3.972174 \\
\hline & 0.000000 & 0.000000 & 0.850300 \\
\hline & 0.931300 & 1.145435 & 5.029769 \\
\hline & 2.313201 & 3.081982 & 4.247049 \\
\hline & -2.313201 & -3.081982 & 4.247049 \\
\hline & -0.931300 & -1.145435 & 5.029769 \\
\hline & -2.225409 & 2.529274 & -2.040085 \\
\hline & -0.769360 & 0.835066 & -1.304210 \\
\hline & -0.493957 & 0.526541 & -2.646102 \\
\hline & -1.174996 & 1.237617 & -3.6 \\
\hline & 0.493957 & -0.526541 & -2.646102 \\
\hline & 0.769360 & -0.835066 & -1.304210 \\
\hline & 2.225409 & -2.529274 & -2.040085 \\
\hline & 1.174996 & -1.237617 & -3.626663 \\
\hline & 0.000000 & 0.000000 & -0.505032 \\
\hline & -1.029875 & 1.024202 & -4.682338 \\
\hline & 1.029875 & -1.024202 & -4.682338 \\
\hline & 1.411050 & 1.911717 & 1.221949 \\
\hline & -1.411050 & -1.911717 & 1.221949 \\
\hline & 3.013837 & 4.760639 & 2.687777 \\
\hline & -3.013837 & -4.760639 & 2.687777 \\
\hline & -3.611746 & 5.400309 & -0.327265 \\
\hline & 3.611746 & -5.400309 & -0.327265 \\
\hline & -3.698300 & 8.474774 & -2.001811 \\
\hline & 3.698300 & -8.474774 & -2.001811 \\
\hline & 2.532471 & 9.481800 & -0.527057 \\
\hline & -2.532471 & -9.481800 & -0.527057 \\
\hline & 0.000000 & 11.084415 & 1.163322 \\
\hline & 0.000000 & -11.084415 & 1.163322 \\
\hline & 3.345725 & 5.999488 & 2.401615 \\
\hline & 3.118476 & 8.326523 & -0.293602 \\
\hline & -1.292164 & 11.034525 & 0.918736 \\
\hline & -3.167672 & 9.656124 & -1.764993 \\
\hline & -3.168998 & 4.182628 & -0.568559 \\
\hline & -2.016379 & 2.213037 & -3.330064 \\
\hline & -1.645177 & 1.854728 & -0.968083 \\
\hline & -3.513539 & 4.501749 & -2.911624 \\
\hline & -3.450555 & 9.339937 & 0.584034 \\
\hline & -0.878054 & 11.178431 & -1.424370 \\
\hline & 3.073174 & 8.755305 & 2.054338 \\
\hline & 3.092613 & 5.554728 & 0.067334 \\
\hline & -3.345725 & -5.999488 & 2.401615 \\
\hline & -3.118476 & -8.326523 & -0.293602 \\
\hline & 1.292164 & -11.034525 & 0.918736 \\
\hline & 3.167672 & -9.656124 & -1.764 \\
\hline & 3.168998 & -4.182628 & -0.56855 \\
\hline
\end{tabular}

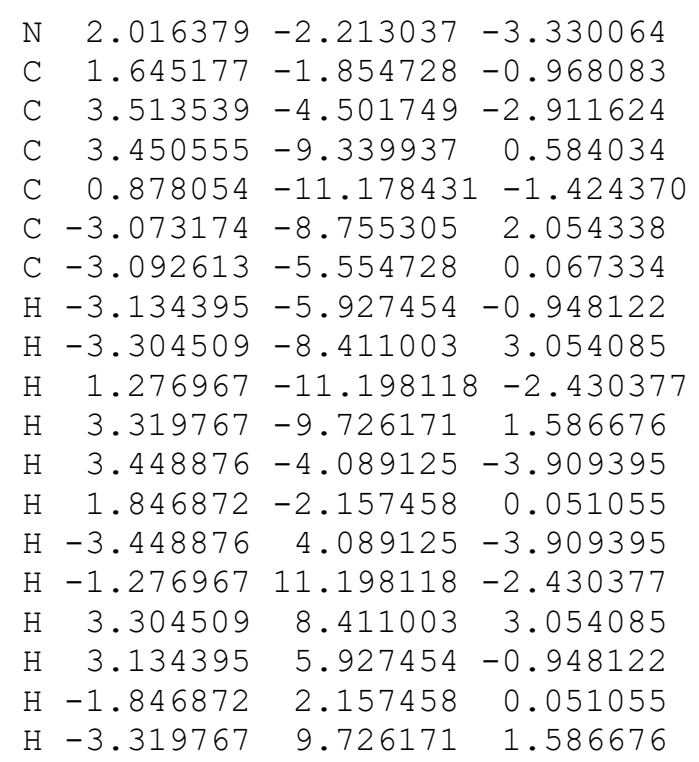

$\Delta \mathrm{H}=-3724.984697$

\section{NN-7}




\begin{tabular}{|c|c|c|c|}
\hline $\mathrm{H}$ & 5.836023 & -6.172066 & 1.239693 \\
\hline & 5.802749 & -5.228647 & -3.363648 \\
\hline & 5.480901 & -13.054049 & -2.366433 \\
\hline & -5.989468 & 6.409845 & -0.896485 \\
\hline & -6.071458 & 6.849864 & 0.429461 \\
\hline & -6.552132 & 8.971089 & -0.503061 \\
\hline & -6.413140 & 10.448467 & -0.442441 \\
\hline & -5.343717 & 5.123642 & -1.261026 \\
\hline & -5.258223 & 4.700432 & -2.591946 \\
\hline & -3.721596 & 3.086596 & -1.786504 \\
\hline & -5.691940 & 12.436078 & -1.503494 \\
\hline & -5.196798 & 12.787569 & -0.242741 \\
\hline & -5.836023 & 6.172066 & 1.239693 \\
\hline & -5.802749 & 5.228647 & -3.363648 \\
\hline & -5.480901 & 13.054049 & -2.366433 \\
\hline & -1.630631 & -2.890546 & 2.168406 \\
\hline & -0.572112 & -0.982038 & 1.664129 \\
\hline & -0.367218 & -0.620688 & 3.009965 \\
\hline & -0.881081 & -1.477855 & 3.972516 \\
\hline & -1.524724 & -2.625276 & 3.539900 \\
\hline & 0.367218 & 0.620688 & 3.009965 \\
\hline & 0.572112 & 0.982038 & 1.664129 \\
\hline & 1.630631 & 2.890546 & 2.168406 \\
\hline & 1.524724 & 2.625276 & 3.539900 \\
\hline & 0.881081 & 1.477855 & 3.972516 \\
\hline & 0.000000 & 0.000000 & 0.856043 \\
\hline$\Pi$ & -0.775898 & -1.267707 & 5.031340 \\
\hline & -1.930912 & -3.343497 & 4.238299 \\
\hline 11 & 1.930912 & 3.343497 & 4.238299 \\
\hline & 0.775898 & 1.267707 & 5.031340 \\
\hline U & 2.650580 & -2.079950 & -2.017867 \\
\hline 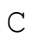 & 0.901741 & -0.687790 & -1.295023 \\
\hline C & 0.577455 & -0.434728 & -2.637730 \\
\hline C & 1.373918 & -1.022573 & -3.613476 \\
\hline C & -0.577455 & 0.434728 & -2.637730 \\
\hline C & -0.9 & 0.687790 & -1.295023 \\
\hline C & -2.650580 & 2.079950 & -2.017867 \\
\hline C & -1.373918 & 1.022573 & -3.613476 \\
\hline $\mathrm{N}$ & 0.000000 & 0.000000 & -0.497587 \\
\hline $\mathrm{H}$ & 1.19 & -0.850593 & -4.670851 \\
\hline $\mathrm{H}$ & -1.191212 & 0.850593 & -4.670851 \\
\hline $\mathrm{C}$ & -4.020773 & 13.678024 & -0.077407 \\
\hline $\mathrm{C}$ & -1.425861 & 14.146802 & 0.051199 \\
\hline $\mathrm{C}$ & 1.425861 & -14.146802 & 199 \\
\hline$C$ & 4.020773 & -13.678024 & -0.077407 \\
\hline $\mathrm{C}$ & -3.496363 & 13.990796 & 1.181504 \\
\hline 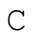 & 3.496363 & -13.990796 & 1.181504 \\
\hline $\mathrm{H}$ & -4.115099 & 13.919158 & 2.066399 \\
\hline $\mathrm{H}$ & 4.115099 & -13.919158 & 2.066399 \\
\hline $\mathrm{N}$ & -1.166217 & -2.065912 & 1.219983 \\
\hline $\mathrm{N}$ & 1.166217 & 2.065912 & 1.219983 \\
\hline $\mathrm{N}$ & 2.639355 & 5.001794 & 2.659019 \\
\hline $\mathrm{V}$ & -2.639355 & -5.001794 & 2.659019 \\
\hline IN & 4.730190 & -4.482740 & -0.269303 \\
\hline & -4.730190 & 4.482740 & -0.269303 \\
\hline & 6.349501 & -7.180041 & -1.921390 \\
\hline & -6.349501 & 7.180041 & -1.921390 \\
\hline
\end{tabular}

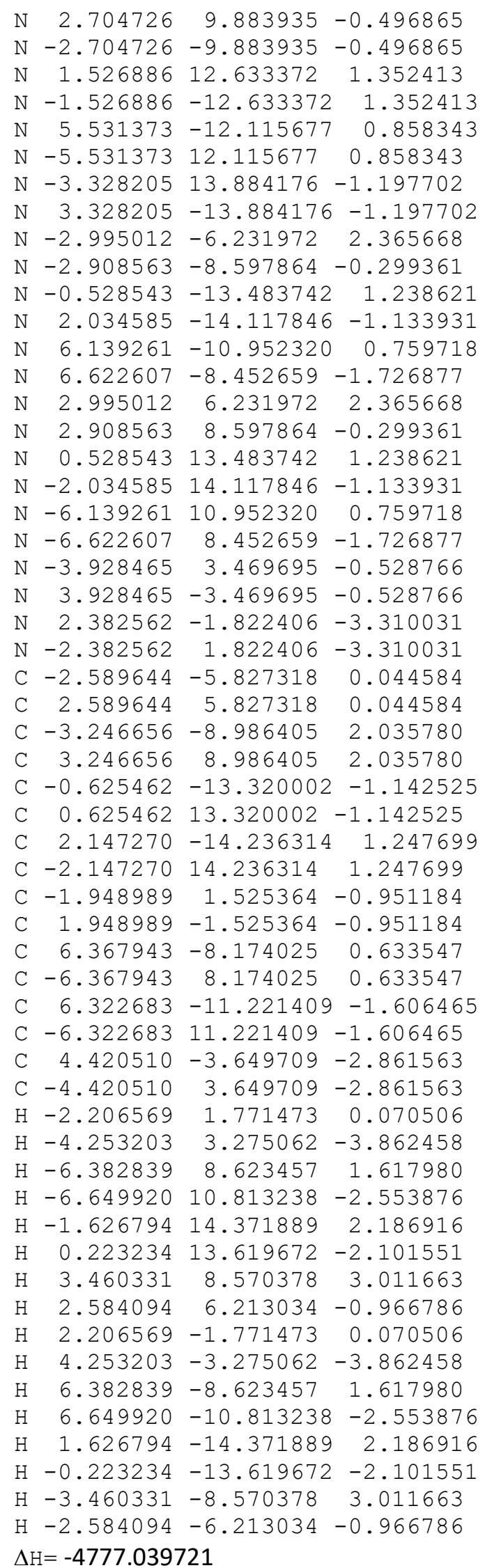

$\mathrm{N}-2.704726-9.883935-0.496865$

N $1.526886 \quad 12.633372 \quad 1.352413$

$\begin{array}{llll}\mathrm{N} & -5.531373 & 12.115677 & 0.858343\end{array}$

$\mathrm{N}-3.328205 \quad 13.884176-1.197702$

$\mathrm{N} \quad 3.328205-13.884176-1.197702$

$\mathrm{N}-2.995012-6.231972 \quad 2.365668$

$\mathrm{N}-2.908563-8.597864-0.299361$

$\mathrm{N}-0.528543-13.483742 \quad 1.238621$

$\mathrm{N} \quad 2.034585-14.117846-1.133931$

$\begin{array}{llll}\mathrm{N} & 2.995012 & 6.231972 & 2.365668\end{array}$

$\mathrm{N} \quad 2.908563 \quad 8.597864 \quad-0.299361$

$\begin{array}{llll}\mathrm{N} & 0.528543 & 13.483742 & 1.238621\end{array}$

$\mathrm{N}-2.034585 \quad 14.117846-1.133931$

$\begin{array}{llll}\mathrm{N}-6.139261 & 10.952320 & 0.759718\end{array}$

$\mathrm{N}-6.622607 \quad 8.452659-1.726877$

$\mathrm{N}-3.928465 \quad 3.469695-0.528766$

$3.928465-3.469695-0.528766$

$\mathrm{N} \quad 2.382562-1.822406-3.310031$

$\mathrm{N}-2.382562 \quad 1.822406-3.310031$

$\begin{array}{llll}\mathrm{C} & -2.589644 & -5.827318 & 0.044584\end{array}$

$\begin{array}{lrrr}\text { C } & 2.589644 & 5.827318 & 0.044584\end{array}$

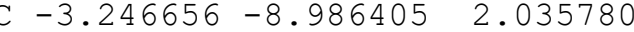

$\begin{array}{llll}\text { C } \quad 3.246656 & 8.986405 & 2.035780\end{array}$

C $-0.625462-13.320002-1.142525$

C $0.62546213 .320002-1.142525$

C $2.147270-14.236314 \quad 1.247699$

$\begin{array}{llll}\text { C } & -2.147270 & 14.236314 & 1.247699\end{array}$

C $-1.948989 \quad 1.525364-0.951184$

C $\quad 1.948989-1.525364-0.951184$

$\begin{array}{llll}\text { C } & 6.367943 & -8.174025 & 0.633547\end{array}$

$\begin{array}{llll}\text { C }-6.367943 & 8.174025 & 0.633547\end{array}$

C $6.322683-11.221409-1.606465$

$-6.32268311 .221409-1.606465$

$4.420510-3.649709-2.861563$

$\mathrm{H}-2.206569 \quad 1.771473 \quad 0.070506$

$\mathrm{H}-4.253203 \quad 3.275062-3.862458$

$\begin{array}{llll}-6.382839 & 8.623457 & 1.617980\end{array}$

$\mathrm{H}-6.649920 \quad 10.813238-2.553876$

$\mathrm{H}-1.626794 \quad 14.371889 \quad 2.186916$

H $\quad 0.223234 \quad 13.619672 \quad-2.101551$

$\mathrm{H} \quad 3.460331 \quad 8.570378 \quad 3.011663$

H $2.584094 \quad 6.213034-0.966786$

H $2.206569-1.771473 \quad 0.070506$

$\mathrm{H} \quad 4.253203-3.275062-3.862458$

H $\quad 6.382839-8.623457 \quad 1.617980$

H $\quad 6.649920-10.813238-2.553876$

H $\quad 1.626794-14.371889 \quad 2.186916$

$-0.223234-13.619672-2.101551$

$\mathrm{H}-2.584094-6.213034-0.966786$

$\Delta \mathrm{H}=-4777.039721$ 


\section{$9 \mathrm{CC}-5$}

\begin{tabular}{|c|c|c|c|}
\hline $\mathrm{C}$ & 3.088516 & 8.279953 & 1.424341 \\
\hline & 2.472230 & 10.791081 & 0.885843 \\
\hline & 2.606578 & 10.451724 & 2.236498 \\
\hline & 2.883740 & 6.816868 & 1.562053 \\
\hline & 1.952243 & 4.366760 & 3876 \\
\hline & 2.118687 & 4.753582 & 0.680200 \\
\hline & 2.345106 & 11.158699 & 3.01 \\
\hline & 1.835360 & 4.079583 & -0.1 \\
\hline & -3.088516 & -8.279953 & 1.4 \\
\hline & -2.472230 & -10.791081 & 843 \\
\hline- & -2.606578 & -10.451724 & 2 . \\
\hline & -2.88 & -6.816868 & 1.56 \\
\hline $\mathcal{C}$ & -1.952243 & -4.366760 & 2.01 \\
\hline & -2.118687 & -4.753582 & 0.68 \\
\hline & -2.345106 & -11.158699 & 213 \\
\hline & -1.83 & -4.079583 & -0.1 \\
\hline 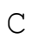 & 2.888906 & -8.418 & -1.4 \\
\hline 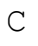 & 3.154930 & -8.863904 & -0.154831 \\
\hline 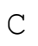 & 2.123173 & -10.872441 & -0.853238 \\
\hline$C$ & 1.009748 & -11.791162 & -0.499477 \\
\hline C & 2.732588 & -6.981517 & -1.783718 \\
\hline C & 2.628379 & -6.534222 & -3.105039 \\
\hline C & 1.865861 & -4.497650 & -2.156707 \\
\hline C & -1.291667 & -12.047228 & $3-0.958288$ \\
\hline $\mathrm{C}$ & -1.452091 & -11.753867 & 0.399070 \\
\hline $\mathrm{H}$ & 3.545342 & -8.181609 & 0.589014 \\
\hline $\mathrm{H}$ & 2.847758 & -7.205078 & -3.925580 \\
\hline $\mathrm{H}$ & -2.143040 & -12.080897 & -1.625435 \\
\hline C & -2.888906 & 8.418336 & -1.452968 \\
\hline $\mathrm{C}$ & -3.154930 & 8.863904 & -0.154831 \\
\hline C & -2.123173 & 10.872441 & -0.853238 \\
\hline $\mathrm{C}$ & -1.009748 & 11.791162 & -0.499477 \\
\hline C & -2.732588 & 6.981517 & -1.783718 \\
\hline $\mathrm{C}$ & -2.628379 & 6.534222 & -3.105039 \\
\hline C & -1.865861 & 4.497650 & -2.156707 \\
\hline C & 1.291667 & 12.047228 & -0.958288 \\
\hline $\mathrm{C}$ & 1.452091 & 11.753867 & 0.399070 \\
\hline $\mathrm{H}$ & -3.545342 & 8.181609 & 0.5 \\
\hline $\mathrm{H}$ & -2.84 & 7.205078 & -3.925580 \\
\hline $\mathrm{H}$ & 2.143040 & 12.080897 & -1.625435 \\
\hline $\mathrm{C}$ & -1.33 & -3.067577 & 2.3 \\
\hline $\mathrm{C}$ & -0.36 & -1.118685 & 1.7 \\
\hline $\mathrm{C}$ & -0.26 & -0.677491 & 3.080078 \\
\hline $\mathrm{C}$ & -0.69 & -1.502051 & 4.1 \\
\hline $\mathrm{C}$ & -1.239824 & -2.724579 & 3.7 \\
\hline $\mathrm{C}$ & 0.261702 & 0.677 & 3.0 \\
\hline $\mathrm{C}$ & 0.36 & 1.118685 & 1.7 \\
\hline $\mathrm{C}$ & 1.336757 & 3.067577 & 2.3 \\
\hline $\mathrm{C}$ & 1.239824 & 2.724579 & 3.747511 \\
\hline $\mathrm{C}$ & 0.697766 & 1.502051 & 4.1 \\
\hline $\mathrm{H}$ & -0.641872 & -1.198662 & 1603 \\
\hline $\mathrm{H}$ & -1.61 & -3.4195 & 4.4 \\
\hline $\mathrm{H}$ & 1.6 & 3.4195 & 4.4 \\
\hline & 0.6 & 1 . & \\
\hline
\end{tabular}

\begin{tabular}{|c|c|c|c|}
\hline C & 1.262401 & -3.143716 & -2.296662 \\
\hline & 0.316273 & -1.140030 & -1.416407 \\
\hline & 0.233961 & -0.688084 & -2.743230 \\
\hline & 0.640748 & -1.509983 & -3.778879 \\
\hline & -0.233961 & 0.688084 & -2.743230 \\
\hline & -0.316273 & 1.140030 & -1.416407 \\
\hline 0 & -1.262401 & 3.143716 & -2.296662 \\
\hline & -0.640748 & 1.509983 & -3.778879 \\
\hline & 0.587067 & -1.189131 & -4.815823 \\
\hline & -0.587067 & 1.189131 & -4.815823 \\
\hline & -0.902981 & -2.280223 & 1.402939 \\
\hline & 0.902981 & 2.280223 & 1.402939 \\
\hline & -2.324421 & -5.147000 & 3.028385 \\
\hline & 2.324421 & 5.147000 & 3.028385 \\
\hline & 2.498263 & -6.188630 & -0.74 \\
\hline & -2.498263 & 6.188630 & -0.74 \\
\hline & 2.546222 & -9.261323 & -2.42 \\
\hline $\mathbb{N}$ & -2.546222 & 9.261323 & -2.428066 \\
\hline & -2.910788 & -9.988 & -0.0 \\
\hline & 2.910788 & 9.988052 & -0.086237 \\
\hline & -0.44 & -11.9 & 052 \\
\hline & 0.4421 & 11.91 & 1.26 \\
\hline & -2.77 & -6.3 & 2.8 \\
\hline & -3.22 & -8.73 & $0.1 \varepsilon$ \\
\hline & 0.79 & -11.9 & 861 \\
\hline & 2.15 & -10 & -2 . \\
\hline & 2.07 & -4.9 & -0.92 \\
\hline $\mathbb{1}$ & 1.14 & -2.7 & -3 \\
\hline 0 & 0.85 & $-2 \cdot 3$ & $-1 \cdot 1$ \\
\hline $\mathrm{C}$ & 2 . & -5.2 & -3.2 \\
\hline 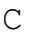 & 2.77 & -10.1 & 383 \\
\hline 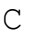 & 0.0 & -12 & -1 . \\
\hline c & -2.92 & -9.14 & 2.51 \\
\hline C & -2 & -6 . & 0 . \\
\hline $\mathrm{N}$ & 2.771572 & 6.36 & 2.80 \\
\hline $\mathrm{N}$ & 3.22 & 8.73 & 0.1 \\
\hline $\mathrm{N}$ & -0.7934 & 11.934020 & 0.81 \\
\hline $\mathrm{N}$ & -2.15 & 10.48 & -2.1 \\
\hline $\mathbb{N}$ & -2.072367 & 4.95 & -0.92 \\
\hline $\mathrm{N}$ & -1.142 & 2.71 & -3.55 \\
\hline $\mathrm{C}$ & -0.855013 & 2.398900 & -1.182689 \\
\hline C & -2.182 & 5.25 & $-3 \cdot 2$ \\
\hline C & -2.773281 & 10.145785 & 0.15 \\
\hline $\mathrm{C}$ & 0.000 & 12.06 & -1.42 \\
\hline $\mathrm{C}$ & 2.920 & 9.143872 & 2.51 \\
\hline C & 2.60 & 6.01 & 0.4 \\
\hline $\mathrm{H}$ & 2.738 & 6.41 & -0.550246 \\
\hline $\mathrm{H}$ & 2.93 & 8.7 & 3.5 \\
\hline & -0.225798 & 12.111639 & -2.486101 \\
\hline $\mathrm{H}$ & -2.832 & 10.54 & 1.1 \\
\hline & -2.04090 & 4.821127 & -4.276350 \\
\hline $\mathrm{H}$ & -0.978239 & 2.812 & -0.19 \\
\hline & 2.040909 & -4.821127 & -4.276350 \\
\hline $\mathrm{H}$ & 0.225798 & -12.111639 & -2.486101 \\
\hline & -2.937163 & -8.748809 & 3.521 \\
\hline & -2.738763 & -6.415838 & -0.550246 \\
\hline & 0.978239 & -2.812764 & -0.192110 \\
\hline
\end{tabular}


$\begin{array}{llcr}\mathrm{H} & 2.832630 & -10.541210 & 1.159215 \\ \mathrm{C} & 0.000000 & 0.000000 & 0.840891 \\ \mathrm{C} & 0.000000 & 0.000000 & -0.515108\end{array}$

$\Delta \mathrm{H}=-3691.669360$

\section{CC- 7}

\begin{tabular}{|c|c|c|c|}
\hline $\mathrm{C}$ & 6.367326 & 6.167422 & 1.393910 \\
\hline & 7.820069 & 8.351790 & 1.003693 \\
\hline & 7.715631 & 7.881293 & 2.317539 \\
\hline & 8.245709 & 9.739254 & 0.695259 \\
\hline & 5.340694 & 5.103227 & 1.531389 \\
\hline & 3.392201 & 3.362097 & 2.030134 \\
\hline & 3.589992 & 3.741240 & 0.697816 \\
\hline & 7.975800 & 12.333825 & 0.278843 \\
\hline & 8.415338 & 10.191476 & -0.617501 \\
\hline & 8.159897 & 8.438406 & 3.132078 \\
\hline & 2.953326 & 3.339009 & -0.08 \\
\hline & 8.536815 & 9.484283 & -1.427 \\
\hline & -6.367326 & -6.167422 & 1.39 \\
\hline & -7.820069 & -8.351790 & 1.00 \\
\hline & -7.715631 & -7.881293 & 2.317539 \\
\hline & -8.245709 & -9.739254 & 0.695259 \\
\hline & -5.340694 & -5.103227 & 1.53 \\
\hline & -3.392201 & -3.362097 & 2.0 \\
\hline & -3.589992 & -3.741240 & 0.697816 \\
\hline & -7.975800 & -12.333825 & $0.2^{7}$ \\
\hline & -8.415338 & -10.191476 & -0 \\
\hline & -8.159897 & -8.438406 & 3.1 \\
\hline & -2.953326 & -3.339009 & -0.080268 \\
\hline & -8.536815 & -9.484283 & -1.42 \\
\hline & 0.064727 & -8.934824 & -1.320718 \\
\hline & 0.000000 & -9.402156 & -0.003424 \\
\hline 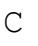 & -0.530872 & -11.497121 & -0 \\
\hline 乙 & -1.260610 & -12.788137 & -0.89 \\
\hline & 0.135937 & -7.487245 & -1.642839 \\
\hline 乙 & 0.253237 & -7.043603 & -2.965044 \\
\hline & 0.015931 & -4.864475 & -2.062785 \\
\hline$C$ & -2.847987 & -14.241750 & -1.880425 \\
\hline C & -3.333767 & -14.393918 & -0.576964 \\
\hline $\mathrm{H}$ & 0.130637 & -8.713478 & 0.821263 \\
\hline $\mathrm{H}$ & 0.368538 & -7.761293 & -3.766673 \\
\hline $\mathrm{H}$ & -3.379955 & -14.671485 & -2.719168 \\
\hline $\mathrm{C}$ & -0.064727 & 8.934824 & -1.320718 \\
\hline $\mathrm{C}$ & 0.000000 & 9.402156 & -0.003424 \\
\hline C & 0.530872 & 11.497121 & -0.97 \\
\hline c & 1.260610 & 12.788137 & -0.897758 \\
\hline $\mathrm{C}$ & -0.135937 & 7.487245 & -1.64 \\
\hline C & -0.253237 & 7.043603 & -2.965044 \\
\hline $\mathrm{C}$ & -0.015931 & 4.864475 & -2.06278 \\
\hline $\mathrm{C}$ & 2.847987 & 14.241750 & -1.88042 \\
\hline 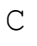 & 3.333767 & 14.393918 & -0.57696 \\
\hline H & -0.130637 & 8.713478 & 0.821263 \\
\hline & -0.368538 & 7.761293 & -3.76 \\
\hline & 3.379955 & 14.671485 & -2.719168 \\
\hline & -2.331312 & -2.401714 & 2.4 \\
\hline
\end{tabular}

\begin{tabular}{|c|c|c|c|}
\hline $\mathrm{C}$ & -0.734733 & -0.915947 & 1.803577 \\
\hline $\mathrm{C}$ & -0.481984 & -0.544991 & 3.135502 \\
\hline C & -1.178140 & -1.177555 & 4.150822 \\
\hline & -2.116916 & -2.128577 & 639 \\
\hline C & 0.481984 & 0.544991 & 3.1 \\
\hline & 0.734733 & 0.915947 & 1.80 \\
\hline C & 2.331312 & 2.401714 & 2.432840 \\
\hline C & 2.116916 & 2.128577 & 639 \\
\hline C & 1.178140 & 1.177555 & 4.1 \\
\hline & -1.018424 & -0.924779 & 3768 \\
\hline & -2.713834 & -2.649030 & 4.524955 \\
\hline F & 2.713834 & 2.649030 & 4.52 \\
\hline $\mathrm{H}$ & 1.018424 & 0.924779 & 5. \\
\hline$C$ & -0.066144 & -3.387287 & -2.22 \\
\hline C & -0.151761 & -1.170809 & -1 \\
\hline $\mathrm{C}$ & -0.057698 & -0.725661 & -2 \\
\hline C & -0.018 & -1.648268 & -3 \\
\hline C & 0.057698 & 0.725661 & $-2 \cdot 6$ \\
\hline C & 0.1 & 1.17 & -1 \\
\hline C & 0.066144 & 3.38 & $-2 \cdot 2$ \\
\hline C & 0.01 & 1.64 & -3.7 \\
\hline $\mathrm{H}$ & 0.045 & -1.33 & $-4 \cdot 7$ \\
\hline $\mathrm{H}$ & -0.04 & 1.33 & -4 \\
\hline C & 4.773 & 14.61 & -0.2 \\
\hline C & 7.14 & 13.55 & 0.1 \\
\hline C & -7.14 & -13.55 & 88 \\
\hline C & -4.77 & $-14 \cdot 6$ & -0 \\
\hline C & 5.26 & 14.68 & 1.0 \\
\hline C & -5.26 & $-14 \cdot 6$ & 1. \\
\hline & & 15.0 & 1.82 \\
\hline $\mathrm{H}$ & -4.63 & $-15 \cdot 0$ & 1. \\
\hline & -1 & $-1 \cdot 8$ & 1.4 \\
\hline $\mathrm{N}$ & 1.64 & 1.81 & 1.4 \\
\hline $\mathrm{N}$ & 4.1 & & \\
\hline $\mathrm{N}$ & -4.152 & -3.82 & 3.02 \\
\hline $\mathrm{N}$ & -0 & -6 . & -0 . \\
\hline $\mathrm{N}$ & 0.007 & 6.65 & -0.6 \\
\hline $\mathrm{N}$ & -0.01 & -9.75 & $-2 \cdot 3$ \\
\hline $\mathrm{N}$ & 0.01 & 9.75 & $-2 \cdot 3$ \\
\hline $\mathrm{N}$ & 7.33 & 7.6 & -0 . \\
\hline & -7.337 & -7.674509 & -0.03 \\
\hline $\mathrm{N}$ & 8.223 & 10.57 & 1.7 \\
\hline T & -8.223 & -10.577386 & 1253 \\
\hline $\mathrm{N}$ & -2.63 & -13.986710 & 0. \\
\hline 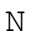 & 2.637 & 13.986710 & $0.4 \varepsilon$ \\
\hline 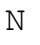 & 5.57 & 14.35 & $-1 \cdot 32$ \\
\hline T & -5.572823 & -14.355670 & $-1 \cdot 3$ \\
\hline $\mathrm{N}$ & -5.10 & -4.691678 & 2.7 \\
\hline T & -6.618 & -6.587512 & 0.15 \\
\hline . & -8.08 & -11.869462 & 1.5 \\
\hline T & -6.7595 & -13.828042 & $-1 \cdot 11$ \\
\hline $\mathrm{N}$ & -1.600 & -13.191330 & 0.32 \\
\hline $\mathrm{N}$ & -0.324842 & -11.020520 & -2.1 \\
\hline $\mathrm{N}$ & 5.109832 & 4.691678 & 2.775 \\
\hline 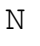 & 6.618 & 6.587512 & 0.15 \\
\hline $\mathrm{N}$ & 8.081 & 11.8 & 1.52 \\
\hline 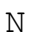 & 6.7595 & 13.828042 & -1.11 \\
\hline
\end{tabular}




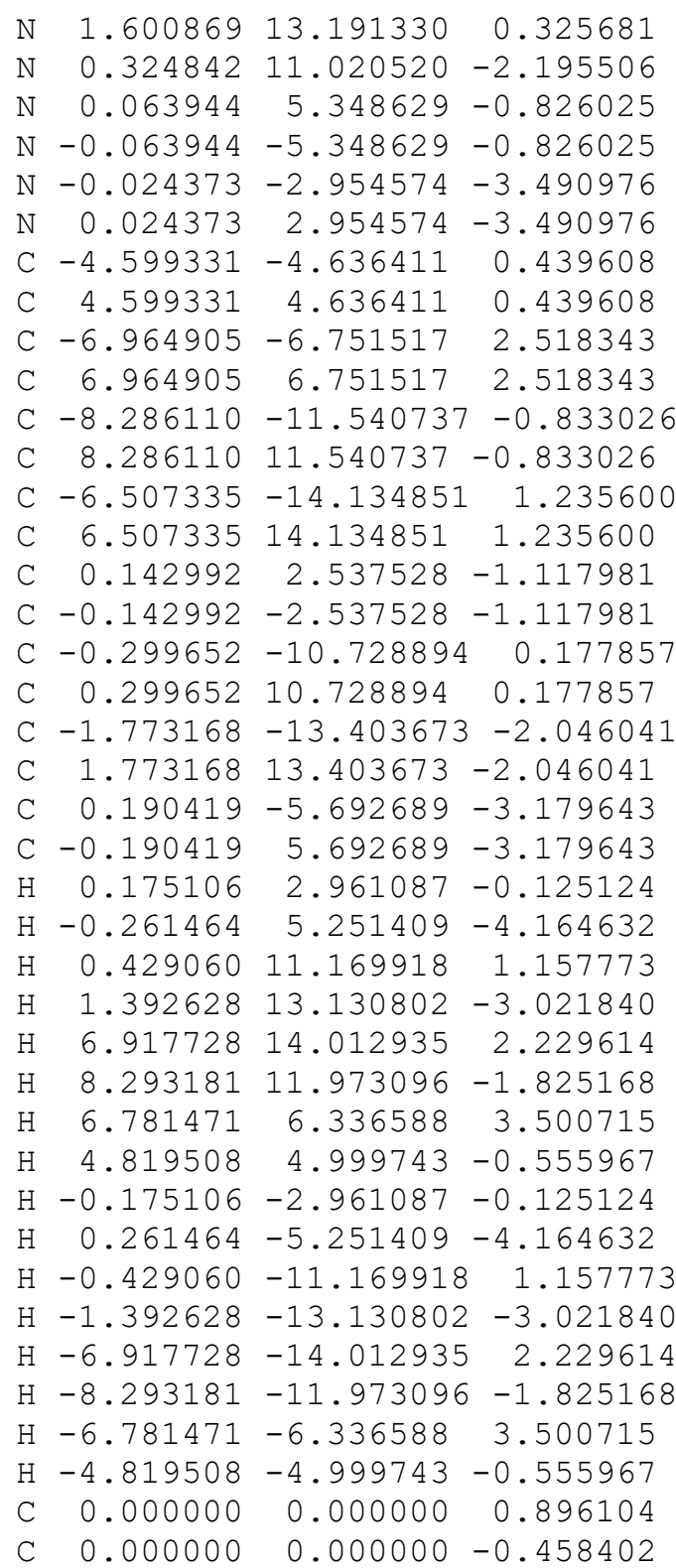

$\Delta \mathrm{H}=-4743.724724$

\section{$10 \mathrm{NN}-5$}

$\begin{array}{rrrr}\text { C } & 0.144011 & 7.922508 & 3.622495 \\ \text { C } & 1.524840 & 8.143613 & 3.632305 \\ \text { C } & 1.007611 & 10.132675 & 2.463559 \\ \text { C } & -0.442871 & 6.560327 & 3.656636 \\ \text { C } & -1.824543 & 6.350254 & 3.712894 \\ \text { C } & -1.337631 & 4.192867 & 2.860116 \\ \text { H } & 2.199525 & 7.377637 & 3.991803 \\ \text { H } & -2.487842 & 7.156490 & 3.998043 \\ \text { C } & -2.049642 & -7.494614 & -3.590854 \\ \text { C } & -1.989528 & -9.914577 & -2.534144 \\ \text { C } & -3.244165 & -9.419826 & -2.906017 \\ \text { C }-1.976551 & -6.011794 & -3.587603\end{array}$

\begin{tabular}{|c|c|c|c|}
\hline $\mathrm{C}$ & -2.137381 & -3.481819 & -2.793599 \\
\hline $\mathrm{C}$ & -0.859674 & -4.041678 & -2.896241 \\
\hline $\mathrm{H}$ & -4.147116 & -9.960296 & -2.653281 \\
\hline $\mathrm{H}$ & 0.008409 & -3.470404 & -2.592920 \\
\hline $\mathrm{C}$ & -0.144011 & -7.922508 & 3.622495 \\
\hline $\mathrm{C}$ & -1.524840 & -8.143613 & 3.63 \\
\hline $\mathrm{C}$ & -1.007611 & -10.132675 & 2. \\
\hline C & -1.316297 & -10.901197 & 1.2 \\
\hline 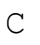 & 0.442871 & -6.560327 & 3.656636 \\
\hline $\mathrm{C}$ & 1.824543 & -6.350254 & 3.712894 \\
\hline $\mathrm{C}$ & 1.337631 & -4.192867 & 2.860116 \\
\hline $\mathrm{C}$ & -0.539613 & -11.269452 & -0.97 \\
\hline $\mathrm{C}$ & -1.798139 & -10.795777 & -1 \\
\hline $\mathrm{H}$ & -2.199525 & -7.377637 & 3.9918 \\
\hline $\mathrm{H}$ & 2.487842 & -7.156490 & 3.998043 \\
\hline $\mathrm{H}$ & 0.236758 & -11.436259 & -1.7 \\
\hline $\mathrm{C}$ & 2.049642 & 7.494614 & -3.59 \\
\hline $\mathrm{C}$ & 1.989528 & 9.914577 & -2.534144 \\
\hline $\mathrm{C}$ & 3.244165 & 9.419826 & $-2 \cdot 9$ \\
\hline $\mathrm{C}$ & 1.798139 & 10.795777 & $-1 \cdot 3$ \\
\hline $\mathrm{C}$ & 1.97 & 6.01 & -3.5 \\
\hline $\mathrm{C}$ & 2.137381 & 3.481819 & $-2 \cdot 7$ \\
\hline $\mathrm{C}$ & 0.85 & 4.04 & -2.8 \\
\hline C & 1.316 & 10.901197 & 1.22 \\
\hline $\mathrm{C}$ & 0.53 & 11.26 & -0.9 \\
\hline $\mathrm{H}$ & 4.147 & 9.960296 & $-2 \cdot 6$ \\
\hline $\mathrm{H}$ & -0.00 & 3.47 & -2.5 \\
\hline $\mathrm{H}$ & -0.23 & 11.436259 & $-1 \cdot 7$ \\
\hline $\mathrm{C}$ & -2.38 & -2.200681 & -2.0 \\
\hline $\mathrm{C}$ & -1.602 & -0.600076 & -0.7 \\
\hline $\mathrm{C}$ & -2.87 & -0.04 & -0.4 \\
\hline $\mathrm{C}$ & -3.94 & -0.617376 & $-1 \cdot 1$ \\
\hline $\mathrm{C}$ & -3.68 & -1.708260 & -1.96 \\
\hline $\mathrm{C}$ & -2.69 & 033700 & \\
\hline $\mathrm{C}$ & -1.316 & 1.113761 & 0.7 \\
\hline $\mathrm{C}$ & -1.75 & 2.979944 & 2.1 \\
\hline $\mathrm{C}$ & -3.536640 & 1.937565 & 1.08 \\
\hline $\mathrm{N}$ & -0.66 & 0.111046 & 0.0 \\
\hline $\mathrm{H}$ & -4.95 & -0.238753 & -1.032561 \\
\hline $\mathrm{H}$ & -4.48 & -2.221882 & $-2 \cdot 4$ \\
\hline $\mathrm{H}$ & -4.611953 & 1.905225 & 0.93364 \\
\hline $\mathrm{C}$ & 1.75 & -2.979944 & 2.1 \\
\hline $\mathrm{C}$ & 1.316297 & -1.113761 & 0.7 \\
\hline $\mathrm{C}$ & 2.69 & -1.033700 & 0.4 \\
\hline $\mathrm{C}$ & 3.53 & -1.937565 & 1.08 \\
\hline $\mathrm{C}$ & 2.87 & 0.045181 & -0 \\
\hline C & 1.60 & 0.600076 & -0.72 \\
\hline $\mathrm{C}$ & 2.38 & 2.200681 & -2.0 \\
\hline $\mathrm{C}$ & 3.689 & 1.708260 & -1.96 \\
\hline $\mathrm{C}$ & 3.94 & 0.617376 & $-1 \cdot 1$ \\
\hline $\mathrm{N}$ & 0.668 & -0.111046 & 0.03 \\
\hline $\mathrm{H}$ & 4.61 & -1.905225 & 0.9 \\
\hline $\mathrm{H}$ & 4.48 & 2.221882 & -2.48720 \\
\hline $\mathrm{H}$ & 4.95 & 0.238753 & -1.032561 \\
\hline $\mathrm{N}$ & $-1 \cdot 3$ & -1.638941 & $-1 \cdot 4^{7}$ \\
\hline $\mathrm{N}$ & 1.3 & 1.638941 & $-1 \cdot 4$ \\
\hline T & -3.2 & -4.120832 & -3.2 \\
\hline
\end{tabular}




$\begin{array}{lrrr}\mathrm{N} & 3.223066 & 4.120832 & -3.230008 \\ \mathrm{~N} & 0.414218 & 5.578973 & 3.380079 \\ \mathrm{~N} & -0.414218 & -5.578973 & 3.380079 \\ \mathrm{~N} & -0.719483 & 8.892669 & 3.319084 \\ \mathrm{~N} & 0.719483 & -8.892669 & 3.319084 \\ \mathrm{~N} & -0.857948 & -9.338889 & -2.944284 \\ \mathrm{~N} & 0.857948 & 9.338889 & -2.944284 \\ \mathrm{~N} & 2.816279 & 10.748690 & -0.492001 \\ \mathrm{~N} & -2.816279 & -10.748690 & -0.492001 \\ \mathrm{~N} & -3.145735 & -5.375382 & -3.613536 \\ \mathrm{~N} & -0.886852 & -8.135052 & -3.475557 \\ \mathrm{~N} & -2.576337 & -10.803199 & 0.800599 \\ \mathrm{~N} & 0.289742 & -9.991218 & 2.735056 \\ \mathrm{~N} & 0.029327 & -4.400973 & 2.989707 \\ \mathrm{~N} & 3.082221 & -2.884879 & 1.890265 \\ \mathrm{C} & 0.816304 & -2.100777 & 1.578065 \\ \mathrm{C} & 2.284904 & -5.126212 & 3.301225 \\ \mathrm{C} & -1.973743 & -9.297638 & 3.039428 \\ \mathrm{C} & -0.289740 & -11.327210 & 0.378286 \\ \mathrm{C} & -3.273816 & -8.160565 & -3.452681 \\ \mathrm{C} & -0.775105 & -5.347078 & -3.313630 \\ \mathrm{~N} & -3.082221 & 2.884879 & 1.890265 \\ \mathrm{~N} & -0.029327 & 4.400973 & 2.989707 \\ \mathrm{~N} & -0.289742 & 9.991218 & 2.735056 \\ \mathrm{~N} & 2.576337 & 10.803199 & 0.800599 \\ \mathrm{~N} & 0.886852 & 8.135052 & -3.475557 \\ \mathrm{~N} & 3.145735 & 5.375382 & -3.613536 \\ \mathrm{C} & -0.816304 & 2.100777 & 1.578065 \\ \mathrm{C} & -2.284904 & 5.126212 & 3.301225 \\ \mathrm{C} & 1.973743 & 9.297638 & 3.039428 \\ \mathrm{C} & 0.289740 & 11.327210 & 0.378286 \\ \mathrm{C} & 3.273816 & 8.160565 & -3.452681 \\ \mathrm{C} & 0.775105 & 5.347078 & -3.313630 \\ \mathrm{H} & -0.238243 & -2.229461 & 1.783735 \\ \mathrm{H} & 0.238243 & 2.229461 & 1.783735 \\ \mathrm{H} & -0.162516 & 5.886132 & -3.360437 \\ \mathrm{H} & 4.198585 & 7.641025 & -3.668369 \\ \mathrm{H} & -3.338051 & 4.885577 & 3.246564 \\ \mathrm{H} & 3.026578 & 9.502499 & 2.894130 \\ \mathrm{H} & -0.695150 & 11.538446 & 0.774435 \\ \mathrm{H} & 0.162516 & -5.886132 & -3.360437 \\ \mathrm{H} & -4.198585 & -7.641025 & -3.668369 \\ \mathrm{H} & 0.695150 & -11.538446 & 0.774435 \\ \mathrm{H} & -3.026578 & -9.502499 & 2.894130 \\ \mathrm{H} & 3.338051 & -4.885577 & 3.246564 \\ \mathrm{~A} \mathrm{H}=-3724.985133 & & \\ & & & \end{array}$

\section{NN-7}

$$
\begin{array}{llrl}
C & -7.470076 & 1.217633 & -4.637810 \\
C & -7.934632 & -0.102473 & -4.640205 \\
C & -10.096677 & 0.858566 & -4.689359 \\
C & -11.517818 & 0.814162 & -4.260410 \\
C & -6.078607 & 1.562792 & -4.251685
\end{array}
$$

$\begin{array}{lrrr}\text { C } & -5.635582 & 2.889866 & -4.229902 \\ \text { C } & -3.761293 & 2.050365 & -3.048487 \\ \text { C } & -13.308817 & 1.894228 & -3.154990 \\ \text { C } & -13.567510 & 0.636023 & -2.60001 \\ \text { H } & -7.234448 & -0.922460 & -4.546973 \\ \text { H } & -6.252995 & 3.673136 & -4.649758 \\ \text { H } & -13.862222 & 2.763068 & -2.823357 \\ \text { C } & 7.354597 & 0.612769 & 4.594112 \\ \text { C } & 9.966856 & 0.179610 & 4.704036 \\ \text { C } & 9.523535 & 1.477211 & 4.983461 \\ \text { C } & 11.365333 & -0.102485 & 4.295107 \\ \text { C } & 5.947900 & 0.806234 & 4.159326 \\ \text { C } & 3.656097 & 1.438592 & 2.968933 \\ \text { C } & 4.002066 & 0.083750 & 3.020686 \\ \text { C } & 13.443924 & -0.353185 & 2.680382 \\ \text { C } & 11.818033 & -1.401477 & 4.040103 \\ \text { H } & 10.238593 & 2.271138 & 5.155418 \\ \text { H } & 3.365290 & -0.654170 & 2.549569 \\ \text { H } & 11.264727 & -2.254762 & 4.409960 \\ \text { C } & 7.470076 & -1.217633 & -4.637810 \\ \text { C } & 7.934632 & 0.102473 & -4.640205 \\ \text { C } & 10.096677 & -0.858566 & -4.689359 \\ \text { C } & 11.517818 & -0.814162 & -4.260410 \\ \text { C } & 6.078607 & -1.562792 & -4.251685 \\ \text { C } & 5.635582 & -2.889866 & -4.229902 \\ \text { C } & 3.761293 & -2.050365 & -3.048487 \\ \text { C } & 13.308817 & -1.894228 & -3.154990 \\ \text { C } & 13.567510 & -0.636023 & -2.600001 \\ \text { H } & 7.234448 & 0.922460 & -4.546973 \\ \text { H } & 6.252995 & -3.673136 & -4.649758 \\ \text { H } & 13.862222 & -2.763068 & -2.823357 \\ \text { C } & -7.354597 & -0.612769 & 4.594112 \\ \text { C } & -9.966856 & -0.179610 & 4.704036 \\ \text { C } & -9.523535 & -1.477211 & 4.983461 \\ \text { C } & -11.365333 & 0.102485 & 4.295107 \\ \text { C } & -5.947900 & -0.806234 & 4.159326 \\ \text { C } & -3.656097 & -1.438592 & 2.968933 \\ \text { C } & -4.002066 & -0.083750 & 3.020686 \\ \text { C } & -13.443924 & 0.353185 & 2.680382 \\ \text { C } & -11.818033 & 1.401477 & 4.040103 \\ \text { H } & -10.238593 & -2.271138 & 5.155418 \\ \text { H } & -3.365290 & 0.654170 & 2.549569 \\ \text { H } & -11.264727 & 2.254762 & 4.409960 \\ \text { C } & 2.504977 & 1.924846 & 2.164277 \\ \text { C } & 0.850245 & 1.462378 & 0.729670 \\ \text { C } & 0.527711 & 2.813086 & 0.503195 \\ \text { C } & 1.261284 & 3.756759 & 1.208084 \\ \text { C } & 2.260193 & 3.299541 & 2.052021 \\ \text { C }-0.546888 & 2.831552 & -0.460888 \\ \text { C } & -0.850245 & 1.494866 & -0.774410 \\ \text { C } & -2.564691 & 2.258540 & -2.189195 \\ \text { C } & -1.284133 & 3.824529 & -1.094821 \\ \text { N } & 0.012612 & 0.676796 & -0.064035 \\ \text { H } & 1.071585 & 4.819005 & 1.099523 \\ \text { H } & 2.881173 & 3.981822 & 2.615644 \\ \text { H } & -1.084175 & 4.877415 & -0.913904 \\ \text { C } & 2.564691 & -2.258540 & -2.189195\end{array}$




\begin{tabular}{|c|c|c|c|}
\hline & 45 & -1.494866 & -0.774410 \\
\hline & 0.546888 & -2.831552 & -0.460888 \\
\hline & 1.284133 & -3.824529 & -1.094821 \\
\hline & -0.527711 & -2.813086 & 0.503195 \\
\hline & -0.850245 & -1.462378 & 0.729670 \\
\hline & -2.504977 & -1.924846 & 2.164277 \\
\hline & -2.260193 & -3.299541 & 2.052021 \\
\hline & -1.261284 & -3.756759 & 1.208084 \\
\hline & -0.012612 & -0.676796 & -0.0 \\
\hline & 1.084175 & -4.877415 & -0.913904 \\
\hline & -2.881173 & -3.981822 & 2.615644 \\
\hline & -1.071585 & -4.819005 & 1.099523 \\
\hline & -14.139465 & 0.349321 & 1.367867 \\
\hline & -14.203954 & 0.477697 & -1.268456 \\
\hline & 14.13 & -0.349321 & 1.367867 \\
\hline & & -0.4 & -1.26845 \\
\hline & 417853 & $3-0$ & $3-0$. \\
\hline & 14.417853 & & -0.69110 \\
\hline & 82166 & -1 & -1.311217 \\
\hline & 14.4 & 1.6 & -1 \\
\hline & 1.7 & 0.9 & 1. \\
\hline & -1.7 & -0.9 & 1.5 \\
\hline & 4.380791 & 2.376959 & 3.5 \\
\hline & -4. & -2.3 & \\
\hline & 5.338651 & -0.5 & -3 \\
\hline & -5.33 & 57 & -3 \\
\hline & 8.28 & -2.2 & -4 . \\
\hline & -8.282667 & 2.25 & -4.83 \\
\hline & 9.125994 & -0.842342 & 4.558232 \\
\hline & -9.125994 & 0.8 & 4. \\
\hline & 12.0 & 0.9 & 3. \\
\hline & -12.078204 & -0.9 & 961 \\
\hline & 12.989140 & 0.471341 & -3.063763 \\
\hline & -12.989140 & -0.471341 & -3.063763 \\
\hline & -14.253693 & 1.598909 & -0.548873 \\
\hline & 14.253693 & -1.598909 & -0.548873 \\
\hline & 5.5 & 2.0 & 69 \\
\hline & 7.8 & -0. & 37 \\
\hline & 13.1 & 0.8 & 232 \\
\hline & 14.221344 & -1.535168 & 5134 \\
\hline & 11.970281 & 371 & -3.892451 \\
\hline & 9.586439 & -2.0 & -4.8 \\
\hline & 5776 & -0.7 & -3.1 \\
\hline & 2.2 & -3.5 & -1 \\
\hline & -4.18 & 0.7 & -3. \\
\hline & -9.586439 & 2.076829 & -4.853901 \\
\hline & -11.970281 & -0.383371 & -3.892451 \\
\hline N & 4.221344 & 1.535168 & 0.765134 \\
\hline & -13.110131 & -0.847348 & 232 \\
\hline & -7.827716 & 0.627848 & 4.505337 \\
\hline & -5.515705 & -2.065033 & 4.1 \\
\hline & -5.184324 & 0.244049 & 3.63717 \\
\hline & .171354 & -1.700668 & 4.92726 \\
\hline & 890112 & 5 & \\
\hline & & -0 & 0.67 \\
\hline & 2.2 & 1.986 & -4.016 \\
\hline & & & \\
\hline
\end{tabular}

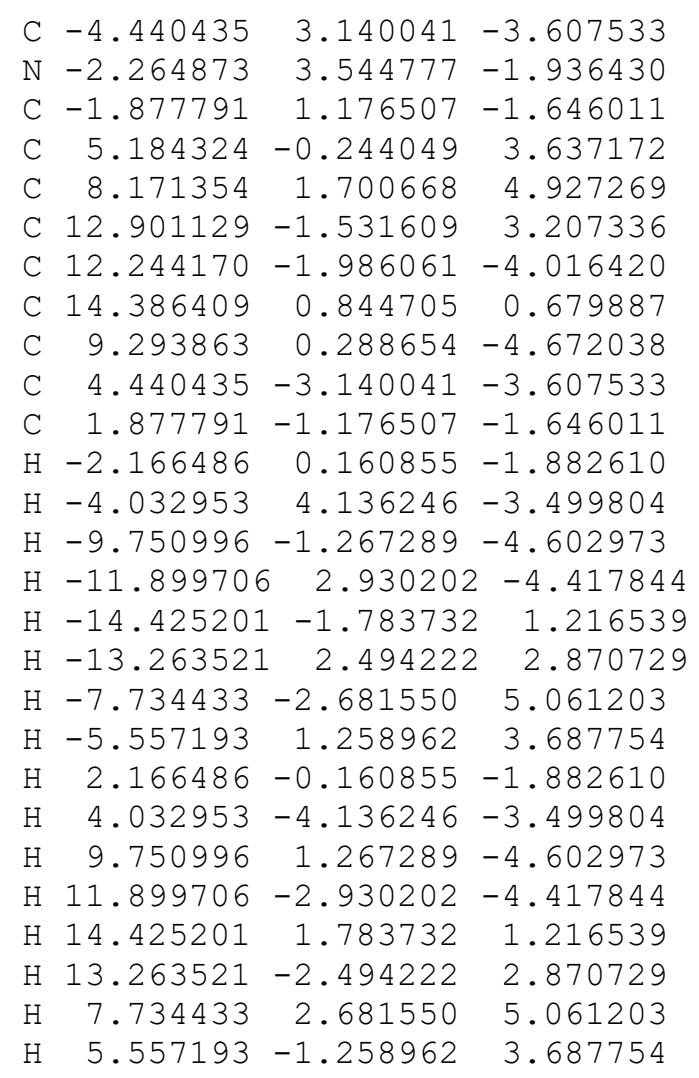

$\Delta \mathrm{H}=-4777.039957$

\section{$10 \mathrm{CC}-5$}

$\begin{array}{llcl}\text { C } & 8.333120 & 1.734700 & 3.029045 \\ \text { C } & 8.788470 & 0.442941 & 3.309163 \\ \text { C } & 10.812202 & 1.164479 & 2.324195 \\ \text { C } & 6.896639 & 2.046616 & 2.835339 \\ \text { C } & 6.435479 & 3.361760 & 2.714678 \\ \text { C } & 4.431671 & 2.384895 & 1.903276 \\ \text { H } & 8.106401 & -0.308740 & 3.684688 \\ \text { H } & 7.089715 & 4.191676 & 2.948396 \\ \text { C } & -8.362424 & 1.147530 & -2.941828 \\ \text { C } & -10.852431 & 0.579239 & -2.268720 \\ \text { C } & -10.532167 & 1.933794 & -2.410289 \\ \text { C } & -6.896639 & 1.303207 & -2.772635 \\ \text { C } & -4.428841 & 1.787380 & -1.906134 \\ \text { C } & -4.805442 & 0.448549 & -2.053949 \\ \text { H } & -11.242222 & 2.702048 & -2.132650 \\ \text { H } & -4.115726 & -0.341045 & -1.782542 \\ \text { C } & -8.333120 & -1.734700 & 3.029045 \\ \text { C } & -8.788470 & -0.442941 & 3.309163 \\ \text { C } & -10.812202 & -1.164479 & 2.324195 \\ \text { C } & -11.760358 & -0.820201 & 1.232678 \\ \text { C } & -6.896639 & -2.046616 & 2.835339 \\ \text { C } & -6.435479 & -3.361760 & 2.714678 \\ \text { C } & -4.431671 & -2.384895 & 1.903276 \\ \text { C } & -12.062297 & -1.279440 & -1.063014 \\ \text { C } & -11.787165 & 0.081072 & -1.228217\end{array}$




\begin{tabular}{|c|c|c|c|}
\hline & 401 & 8740 & 88 \\
\hline & 89715 & -4.191676 & 2.948396 \\
\hline & -12.107309 & -1.946121 & \\
\hline & 8.362424 & -1.147530 & -2.941828 \\
\hline & 10.852431 & -0.579239 & -2.268720 \\
\hline & 10.532167 & -1.933794 & -2.410289 \\
\hline & 11.787165 & -0.081072 & -1.228217 \\
\hline & 6.896639 & -1.303207 & -2.772635 \\
\hline & 4.428841 & -1.7 & \\
\hline & 4.805442 & -0.448549 & -2.053949 \\
\hline & 11.760358 & 0.820201 & 1.232678 \\
\hline & 12.062297 & 1.279440 & -1.063014 \\
\hline & 11.242222 & -2.702048 & -2.132650 \\
\hline & 4.115726 & 0.341045 & -1.782542 \\
\hline & 12.107309 & 6121 & -1.914230 \\
\hline & -3.118123 & 645 & -1.3 \\
\hline & -1.137987 & 1.571929 & -0.402182 \\
\hline & -0.708380 & 2.905838 & -0.320603 \\
\hline & -1.555191 & 3.915655 & -0.742804 \\
\hline & -2.786619 & 3.543611 & -1.2 \\
\hline & 0.657595 & 267 & 836 \\
\hline & 1.121589 & 1.593923 & 0.277641 \\
\hline & 3.091903 & 2.505629 & 1.265055 \\
\hline & 1.458069 & 3.964067 & 0.591829 \\
\hline & -1.262799 & 9513 & -0.702579 \\
\hline & -3.498701 & 427 & -1.6 \\
\hline & 1.126921 & 4.996963 & 0.521920 \\
\hline & -3.091903 & -2.505629 & 1.265055 \\
\hline & -1.121589 & -1.593923 & 0.277641 \\
\hline & -0.657595 & -2.917267 & 0.173836 \\
\hline & -1.458069 & -3.964067 & 829 \\
\hline & 380 & -2.9 & -0.3 \\
\hline & 1.137987 & -1.571929 & $-0.4 c-3 x$ \\
\hline & 3.118123 & -2.185645 & -1.327307 \\
\hline & 2.786619 & -3.543611 & -1.250536 \\
\hline & 1.555191 & -3.915655 & -0.742804 \\
\hline & -1.126921 & -4.9 & 0.52 \\
\hline & 3.4. & -4.2 & -1.6 \\
\hline & 1.262799 & -4.9 & $-0.7-7 \quad y$ \\
\hline & -2.309758 & 1.206834 & -0.905911 \\
\hline $\mathrm{N}$ & 2.309758 & -1.206834 & -0.905911 \\
\hline & -5.229508 & 951 & 4287 \\
\hline & 5.229508 & -2.7 & -2.2 \\
\hline & 6.123224 & 0.992467 & 2.5 \\
\hline & -6.123224 & -0.9 & 2. \\
\hline $\mathrm{N}$ & 9.171890 & 2.719772 & 2.704955 \\
\hline N & -9.171890 & -2.719772 & 2.704955 \\
\hline & -10.047803 & -0.383394 & -2.724760 \\
\hline & 10.047803 & 0.383394 & -2.724760 \\
\hline & 11.931062 & -0.941413 & -0.214057 \\
\hline & -11.931062 & $0 \quad 94141$ & -0.214057 \\
\hline & -6.453211 & 2.553978 & -2.679172 \\
\hline & -8.809358 & -0.101251 & -3.068335 \\
\hline & -11.922228 & 0.48978 & 1.02111 \\
\hline & -10.404618 & -2.433742 & 2.34250 \\
\hline & -4.897253 & -1.159197 & 2.126455 \\
\hline & -2.655120 & -3.761580 & 1.123969 \\
\hline
\end{tabular}

\begin{tabular}{lrrr} 
C & -2.368814 & -1.379803 & 0.848983 \\
C & -5.164219 & -3.533781 & 2.234898 \\
C & -10.083014 & -0.150197 & 2.959331 \\
C & -12.049507 & -1.751016 & 0.228279 \\
C & -9.235221 & 2.226530 & -2.754176 \\
C & -6.075729 & 0.198472 & -2.511658 \\
N & 2.655120 & 3.761580 & 1.123969 \\
N & 4.897253 & 1.159197 & 2.126455 \\
N & 10.404618 & 2.433742 & 2.342504 \\
N & 11.922228 & -0.489780 & 1.021115 \\
N & 8.809358 & 0.101251 & -3.068335 \\
N & 6.453211 & -2.553978 & -2.679172 \\
C & 2.368814 & 1.379803 & 0.848983 \\
C & 5.164219 & 3.533781 & 2.234898 \\
C & 10.083014 & 0.150197 & 2.959331 \\
C & 12.049507 & 1.751016 & 0.228279 \\
C & 9.235221 & -2.226530 & -2.754176 \\
C & 6.075729 & -0.198472 & -2.511658 \\
H & -2.789944 & -0.394684 & 0.990084 \\
H & 2.789944 & 0.394684 & 0.990084 \\
H & 6.469513 & 0.803622 & -2.624218 \\
H & 8.852771 & -3.238909 & -2.779899 \\
H & 4.723751 & 4.509057 & 2.077815 \\
H & 10.489526 & -0.850456 & 3.030340 \\
H & 12.076526 & 2.809407 & 0.453595 \\
H & -6.469513 & -0.803622 & -2.624218 \\
H & -8.852771 & 3.238909 & -2.779899 \\
H & -12.076526 & -2.809407 & 0.453595 \\
H & -10.489526 & 0.850456 & 3.030340 \\
H & -4.723751 & -4.509057 & 2.077815 \\
C & -0.000140 & 0.678025 & -0.055929 \\
C & 0.000140 & -0.678025 & -0.055929 \\
\hline$\Delta H$ & -3691.669171 &
\end{tabular}

$\Delta \mathrm{H}=-3691.669171$

\section{$10 \mathrm{CC}-7$}

$\begin{array}{rrrr}\mathrm{C} & -3.464073 & 7.571639 & 3.514709 \\ \mathrm{C} & -2.291208 & 8.320454 & 3.661395 \\ \mathrm{C} & -3.734305 & 10.164811 & 4.009955 \\ \mathrm{C} & -4.008855 & 11.616577 & 3.862361 \\ \mathrm{C} & -3.454117 & 6.183251 & 2.988844 \\ \mathrm{C} & -4.633977 & 5.436966 & 2.889644 \\ \mathrm{C} & -3.295375 & 3.804474 & 1.811975 \\ \mathrm{C} & -5.422592 & 13.300448 & 2.988456 \\ \mathrm{C} & -4.232513 & 13.930207 & 2.607336 \\ \mathrm{H} & -1.327833 & 7.849921 & 3.512983 \\ \mathrm{H} & -5.562561 & 5.843200 & 3.268693 \\ \mathrm{H} & -6.379115 & 13.694238 & 2.670433 \\ \mathrm{C} & 0.824045 & -8.230597 & -3.469763 \\ \mathrm{C} & 1.815326 & -10.649136 & -3.919561 \\ \mathrm{C} & 0.427753 & -10.499302 & -4.021973 \\ \mathrm{C} & 2.454072 & -11.977863 & -3.753060 \\ \mathrm{C} & 0.359071 & -6.915068 & -2.961250 \\ \mathrm{C} & -0.697627 & -4.720765 & -1.890230 \\ \mathrm{C} & 0.697627 & -4.827412 & -1.892524 \\ \mathrm{C} & 3.390002 & -14.088966 & -2.470287\end{array}$




\begin{tabular}{|c|c|c|c|}
\hline $\mathrm{C}$ & 3.843348 & -12.138391 & -3.725729 \\
\hline $\mathrm{H}$ & -0.200141 & -11.360343 & -4.210727 \\
\hline & 1.301851 & -4.041275 & -1.457232 \\
\hline & 4.490581 & -11.364903 & $3-4.117950$ \\
\hline & 3.464073 & -7.571639 & 3.514709 \\
\hline & 2.291208 & -8.320454 & 3.661395 \\
\hline & 3.734305 & -10.164811 & 4.009955 \\
\hline & 4.008855 & -11.616577 & 3.862361 \\
\hline & 3.454117 & -6.183251 & 2.988844 \\
\hline & 4.633977 & -5.436966 & 2.889644 \\
\hline & 3.295375 & -3.804474 & 1.811975 \\
\hline & 5.422592 & -13.300448 & 2.988456 \\
\hline & 4.232513 & -13.930207 & $2.60^{\circ}$ \\
\hline & 1.327833 & -7.849921 & 3.5129 \\
\hline & 5.562561 & -5.843200 & 3.268 \\
\hline & 6.379115 & -13.694238 & 2.670433 \\
\hline$\checkmark$ & -0.824045 & 8.230597 & -3.469763 \\
\hline $\mathrm{C}$ & -1.815 & 10.649136 & -3.91 \\
\hline C & -0.427753 & 10.499302 & -4.021973 \\
\hline $\mathrm{C}$ & -2.454072 & 11.977863 & -3.75 \\
\hline$C$ & -0.359071 & 6.915068 & -2.961250 \\
\hline C & 0.697627 & 4.720765 & -1.89 \\
\hline & -0.697627 & 4.827412 & -1.892 \\
\hline C & -3.390002 & 14.088966 & -2.47 \\
\hline C & -3.843348 & 12.1 & -3.7 \\
\hline $\mathrm{H}$ & 0.200141 & 11.360343 & -4.210727 \\
\hline & $-1 \cdot 30$ & 4.0412 & -1.45 \\
\hline $\mathrm{H}$ & -4.490581 & 11.364903 & -4.117950 \\
\hline C & -1.40 & -3.54 & $-1 \cdot 315$ \\
\hline C & -1.274628 & -1.461683 & -0.425442 \\
\hline $\mathrm{C}$ & -2.67 & -1.335772 & -0.383 \\
\hline $\mathrm{C}$ & -3.454118 & -2.399346 & -0.801033 \\
\hline 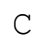 & -2.802567 & -3.526692 & -1.27 \\
\hline $\mathrm{C}$ & -2.995158 & 0.006818 & 0.076201 \\
\hline C & -1.804862 & 0.743568 & 0.207508 \\
\hline C & -3.134454 & 2.486183 & 1.140145 \\
\hline C & -4.199347 & 0.579261 & 0.445505 \\
\hline $\mathrm{H}$ & -4.537731 & -2.350214 & -0.788077 \\
\hline $\mathrm{H}$ & -3.345974 & -4.388346 & -1.633638 \\
\hline $\mathrm{H}$ & -5.138843 & 0.041447 & 0.348387 \\
\hline $\mathrm{C}$ & 3.134454 & -2.486183 & 1.140145 \\
\hline $\mathrm{C}$ & 1.804862 & -0.743568 & 0.207508 \\
\hline $\mathrm{C}$ & 2.995158 & -0.006818 & 0.076201 \\
\hline $\mathrm{C}$ & 4.199347 & -0.579261 & 0.445505 \\
\hline $\mathrm{C}$ & 2.672327 & 1.335772 & -0.383320 \\
\hline $\mathrm{C}$ & 1.274628 & 1.461683 & -0.425442 \\
\hline $\mathrm{C}$ & 1.403446 & 3.545293 & -1.315116 \\
\hline $\mathrm{C}$ & 2.802567 & 3.526692 & -1.270904 \\
\hline $\mathrm{C}$ & 3.454118 & 2.399346 & -0.801033 \\
\hline $\mathrm{H}$ & 5.138843 & -0.041447 & 0.348387 \\
\hline $\mathrm{H}$ & 3.345974 & 4.388346 & -1.633638 \\
\hline $\mathrm{H}$ & 4.537731 & 2.350214 & -0.788077 \\
\hline $\mathrm{C}$ & -3.716983 & 14.825915 & -1.222508 \\
\hline $\mathrm{C}$ & -4.134129 & 14.754062 & 1.376335 \\
\hline $\mathrm{C}$ & 3.716983 & -14.825915 & -1.222508 \\
\hline $\mathrm{C}$ & 4.134129 & 062 & 35 \\
\hline $\mathrm{C}$ & -2.924181 & 15.311292 & 0.94949 \\
\hline
\end{tabular}

\begin{tabular}{|c|c|c|c|}
\hline 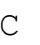 & 2.924181 & -15.311292 & 0.9 \\
\hline $\mathrm{H}$ & -2.150144 & 15.561398 & 1.663158 \\
\hline $\mathrm{H}$ & 2.150144 & -15.561398 & 1.663158 \\
\hline & -0.644812 & -2.530763 & -0.890510 \\
\hline 1 & 0.644812 & 2.530763 & -0.890510 \\
\hline & -1.481259 & -5.660087 & -2.417310 \\
\hline$\Lambda$ & 1.481259 & 5.660087 & -2.417310 \\
\hline 1 & 2.285159 & -5.733557 & 2.54 \\
\hline 1 & -2.285159 & 5.733557 & 2.5 \\
\hline $\mathbb{N}$ & 4.668545 & -8.085584 & 3.75 \\
\hline$N$ & -4.668545 & 8.085584 & 3.7 \\
\hline 1 & 2.632487 & -9.613485 & -3.7 \\
\hline & -2.63 & & -3.7 \\
\hline $\mathrm{N}$ & 1.62 & -12 & -3 \\
\hline & -1.62 & 12.9 & -3.3 \\
\hline $\mathrm{N}$ & 3.04 & -13 & 3.0 \\
\hline 1 & -3.0 & 13.54 & 3. \\
\hline $\mathrm{N}$ & -5.16 & 14.590523 & $0.53 \varepsilon$ \\
\hline $\mathrm{N}$ & 5.1 & -14 & \\
\hline $\mathrm{N}$ & -0.96 & -6.7 & -2.9398 \\
\hline $\mathrm{N}$ & 2.1 & -8.4 & -3.52 \\
\hline $\mathrm{N}$ & 2.0 & -14. & -2 . \\
\hline $\mathrm{N}$ & 4.9 & -14. & -0 . \\
\hline $\mathrm{N}$ & 2.93 & -12.39 & \\
\hline $\mathrm{N}$ & 4.8 & -9.37 & 3.9 \\
\hline $\mathrm{N}$ & 2.2 & -4 & \\
\hline $\mathrm{N}$ & 4.26 & -1.79 & 0.9 \\
\hline $\mathrm{N}$ & -2 . & & \\
\hline N & -4.80 & 9.372 & 3.9 \\
\hline $\mathrm{N}$ & -2.9 & 12. & \\
\hline N & -4.95 & 14.627 & -0.75 \\
\hline 2 & -2 . & 14. & -2.7 \\
\hline $\mathrm{N}$ & -2.140 & 8.411 & -3.5207 \\
\hline $\mathrm{N}$ & 0.96 & 6.7 & $-2 \cdot 9$ \\
\hline $\mathrm{C}$ & -1.243 & 5.957824 & -2.450147 \\
\hline C & 0.08 & 9.24 & -3.7 \\
\hline $\mathrm{C}$ & -4.3301 & 13.240755 & -3.06 \\
\hline $\mathrm{C}$ & -2.70 & 15.3 & -0.4 \\
\hline $\mathrm{C}$ & -5.304 & 12.095 & 3.63 \\
\hline C & -2.43 & 9.6 & 3.92 \\
\hline$C$ & -4.550 & 4.21 & 2.28 \\
\hline $\mathrm{N}$ & -4.26 & 1.79 & 0.9 \\
\hline $\mathrm{C}$ & -1.872 & 2.011175 & 0.76 \\
\hline $\mathrm{C}$ & 1.24 & -5.95 & -2.45 \\
\hline $\mathrm{C}$ & -0.083 & -9.248381 & -3.790118 \\
\hline $\mathrm{C}$ & 4.33 & -13.2 & -3 \\
\hline $\mathrm{C}$ & 5.304 & -12.095950 & 3.6 \\
\hline $\mathrm{C}$ & 2.70 & -15 . & -0 \\
\hline $\mathrm{C}$ & 2.430579 & -9.659757 & 3.925422 \\
\hline $\mathrm{C}$ & 4.55 & -4.2 & 2.2 \\
\hline $\mathrm{C}$ & 1.872828 & -2.011175 & 0.7 \\
\hline $\mathrm{H}$ & -1.001 & 2.6 & 0.9 \\
\hline 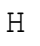 & -5.40 & 3.565179 & 2.1480 \\
\hline 11 & -1.585 & 10.331760 & 3.99720 \\
\hline 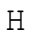 & -6.1 & 11. & 3.8 \\
\hline $\mathrm{H}$ & -1.7 & 0067 & -0.831400 \\
\hline & -5.3 & 13.3 & -2.9012 \\
\hline
\end{tabular}




$\begin{array}{rrrc}\mathrm{H} & 1.145367 & 9.038311 & -3.796461 \\ \mathrm{H} & -2.310470 & 6.136175 & -2.486521 \\ \mathrm{H} & 1.001191 & -2.625651 & 0.935992 \\ \mathrm{H} & 5.406848 & -3.565179 & 2.148068 \\ \mathrm{H} & 1.585193 & -10.331760 & 3.997206 \\ \mathrm{H} & 6.159668 & -11.475631 & 3.872870 \\ \mathrm{H} & 1.752503 & -15.630067 & -0.831400 \\ \mathrm{H} & 5.387980 & -13.396233 & -2.901276 \\ \mathrm{H} & -1.145367 & -9.038311 & -3.796461 \\ \mathrm{H} & 2.310470 & -6.136175 & -2.486521 \\ \mathrm{C} & -0.660205 & -0.151537 & -0.088391 \\ \mathrm{C} & 0.660205 & 0.151537 & -0.088391\end{array}$

\section{$\Delta \mathrm{H}=-4743.724448$}

\section{$\mathbf{E}$}

$\begin{array}{rrrr}\mathrm{C} & -2.781583 & 9.096492 & -0.574347 \\ \mathrm{C} & -2.722623 & 8.708832 & -1.918564 \\ \mathrm{H} & -3.595755 & 8.291725 & -2.405696 \\ \mathrm{C} & -1.524112 & 8.690577 & -2.609158 \\ \mathrm{H} & -1.506582 & 8.297571 & -3.620557 \\ \mathrm{C} & -0.322639 & 9.029446 & -1.977395 \\ \mathrm{C} & -0.418430 & 9.642670 & -0.725825 \\ \mathrm{H} & 0.482270 & 9.965963 & -0.213023 \\ \mathrm{C} & -1.628582 & 9.687499 & -0.044186 \\ \mathrm{H} & -1.635921 & 10.087389 & 0.963654 \\ \mathrm{C} & 0.953712 & 8.435693 & -2.452783 \\ \mathrm{C} & 0.887580 & 7.122518 & -2.928018 \\ \mathrm{H} & -0.076183 & 6.640205 & -3.042485 \\ \mathrm{C} & 2.024503 & 6.368294 & -3.130761 \\ \mathrm{H} & 1.909049 & 5.323305 & -3.392704 \\ \mathrm{C} & 3.297299 & 6.886116 & -2.877276 \\ \mathrm{C} & 3.374437 & 8.223936 & -2.473916 \\ \mathrm{H} & 4.344441 & 8.678822 & -2.297578 \\ \mathrm{C} & 2.225989 & 8.982767 & -2.261770 \\ \mathrm{H} & 2.325648 & 10.008655 & -1.920128 \\ \mathrm{C} & 4.428707 & 5.922980 & -2.850188 \\ \mathrm{C} & 4.399133 & 4.774605 & -3.649854 \\ \mathrm{H} & 3.721446 & 4.724128 & -4.495492 \\ \mathrm{C} & 5.132038 & 3.649818 & -3.311427 \\ \mathrm{H} & 4.978444 & 2.731688 & -3.869504 \\ \mathrm{C} & 5.945673 & 3.640043 & -2.175449 \\ \mathrm{C} & 6.157720 & 4.858221 & -1.528714 \\ \mathrm{H} & 6.850798 & 4.897267 & -0.693840 \\ \mathrm{C} & 5.402923 & 5.978759 & -1.851397 \\ \mathrm{H} & 5.495716 & 6.871723 & -1.240105 \\ \mathrm{C} & 6.302015 & 2.345267 & -1.543527 \\ \mathrm{C} & 6.651844 & 1.205588 & -2.274238 \\ \mathrm{H} & 6.904950 & 1.298545 & -3.326495 \\ \mathrm{C} & 6.637278 & -0.049982 & -1.684114 \\ \mathrm{H} & 6.905871 & -0.923129 & -2.270962 \\ \mathrm{C} & 6.268084 & -0.210962 & -0.345483 \\ \mathrm{C} & 6.017581 & 0.936574 & 0.403734 \\ \mathrm{H} & 5.718511 & 0.848758 & 1.441870 \\ \mathrm{C} & 6.038395 & 2.191657 & -0.179667 \\ \mathrm{H} & 5.748896 & 3.050498 & 0.416622\end{array}$

$\begin{array}{llll}\text { C } 5.988923-1.540830 & 0.241009\end{array}$

C $5.323610-2.499703-0.519901$

H $5.104684-2.301494-1.563006$

$\begin{array}{llll}\text { C } 4.844802 & -3.664745 & 0.052868\end{array}$

$\mathrm{H} \quad 4.266793-4.350708-0.558057$

C $5.003814-3.910853 \quad 1.418794$

C $5.748553-2.993167 \quad 2.166101$

$\mathrm{H} \quad 5.930738-3.180559 \quad 3.220403$

C $6.235865-1.830640 \quad 1.585988$

H $6.812194-1.131101 \quad 2.183825$

$\begin{array}{llll}\text { C } 4.222665 & -5.015044 & 2.027270\end{array}$

C $4.005305-6.209305 \quad 1.339273$

$\begin{array}{llll}\mathrm{H} & 4.589030 & -6.428607 & 0.450441\end{array}$

C $2.981386-7.064782 \quad 1.709340$

H $\quad 2.783377 \quad-7.931067 \quad 1.090540$

C $2.132697-6.773009 \quad 2.780485$

C $2.476974-5.674857 \quad 3.576163$

$\mathrm{H} \quad 1.898736-5.441275 \quad 4.463685$

C $3.494985-4.810156 \quad 3.202821$

$\mathrm{H} \quad 3.656781-3.906781 \quad 3.782481$

C $\quad 0.802151-7.433535 \quad 2.870870$

C $0.517999-8.640310 \quad 2.218605$

$\mathrm{H} \quad 1.326962-9.268786 \quad 1.860919$

C $-0.787656-9.024879 \quad 1.948143$

$\mathrm{H}-0.967559-9.926409 \quad 1.370627$

C $-1.868081-8.235883 \quad 2.349598$

$\begin{array}{llll}\text { C }-1.588894 & -7.134531 & 3.160419\end{array}$

$\mathrm{H}-2.403879-6.503380 \quad 3.500216$

C $-0.285416-6.733818 \quad 3.402806$

$\mathrm{H}-0.127227-5.793736 \quad 3.918880$

C $-3.216576-8.340947 \quad 1.742604$

C $-3.311632-8.456408 \quad 0.355718$

$\mathrm{H}-2.421928-8.665925-0.228502$

C $-4.478330-8.118116-0.308700$

$\mathrm{H}-4.447644-8.082156-1.390405$

C $-5.605020-7.661291 \quad 0.380468$

C $-5.562076-7.736769 \quad 1.778813$

$\mathrm{H}-6.437422-7.479683 \quad 2.365492$

C $-4.389207-8.055546 \quad 2.446939$

$\mathrm{H}-4.371475-8.028650 \quad 3.532517$

C $-6.643194-6.872504-0.337909$

C $-6.751937-6.884817-1.734792$

$\mathrm{H}-6.299326-7.685480-2.309714$

C $-7.359172-5.842405-2.417989$

$\mathrm{H}-7.356997-5.847309-3.503978$

C $-7.903318-4.753218-1.730922$

C $-7.973707-4.842294-0.340796$

$\mathrm{H}-8.380100 \quad-4.015704 \quad 0.232030$

C $-7.355106-5.878543 \quad 0.338371$

$\mathrm{H}-7.314782-5.816612 \quad 1.418482$

C $-8.109837-3.429811-2.365807$

C $-7.124980-2.953904-3.232745$

$\mathrm{H}-6.359728-3.632817-3.595054$

C $-6.998618-1.602731-3.503944$

$\mathrm{H}-6.132329-1.281337-4.069414$

C $-7.865651-0.659929-2.941443$

C $-8.966443-1.165412-2.236785$ 


\begin{tabular}{|c|c|c|c|}
\hline & 723143 & -0.491414 & - \\
\hline & -9.078546 & -2.517125 & -1.94175 \\
\hline & -9.901879 & -2.856960 & -1.32115 \\
\hline & -7.452843 & 0.769479 & \\
\hline & -6.377978 & 1.264677 & \\
\hline & 6.0129 & 0.719025 & \\
\hline & 5.680747 & 2.392166 & \\
\hline & 4.7721. & 2.6 & \\
\hline & 6.044086 & 3.092723 & -2 \\
\hline & 7.248086 & 2.7332 & \\
\hline & -7.604063 & 3.299966 & -0 \\
\hline & 7.9299 & & \\
\hline & -8.7981 & 1.293641 & -1 \\
\hline & $-5.0633^{7}$ & 3.967 & \\
\hline & -2.82522 & & \\
\hline & -3.90 & & \\
\hline & -3.705949 & 8.390627 & \\
\hline & -2.79 & 8.72 & \\
\hline & -4 & & \\
\hline & -4.3662 & 7.4 & $\checkmark$ \\
\hline & -5 . & & \\
\hline & -5 . & & \\
\hline & -6 . & & \\
\hline & -5.10 & 8.1 & -0 \\
\hline & -5 . & & \\
\hline & -6.40 & 5.8 & \\
\hline & -5 . & & \\
\hline & -4.73 & 5.3 & 3 \\
\hline & -6 . & 3.7 & \\
\hline & -5.43 & 3.0 & 4 \\
\hline & -7 . & & \\
\hline & -8.06 & 4.1 & 2 \\
\hline & -9 . & 3.7 & \\
\hline & -7.65 & 5.4 & 1 \\
\hline & -8.28 & 6.0 & \\
\hline & -7.3772 & 1.7 & 2 \\
\hline & -6.67 & 0.8 & \\
\hline & -6.26 & 1.2 & \\
\hline & -6 . & -0.4 & 3. \\
\hline & -5.745 & $-1 \cdot 0$ & 3. \\
\hline & -6.82 & -0.9 & 1 \\
\hline & -7.70 & -0.1 & 1 \\
\hline & -8.10 & -0.4 & 0 \\
\hline & -7.9621 & 1.18 & \\
\hline & -8 . & 1. & \\
\hline & -6.1912 & -2.1 & 1 . \\
\hline & -5 & -3.1 & 2 . \\
\hline & -5.99 & $-3 \cdot 2$ & \\
\hline & -4. & -4 & 1.5 \\
\hline & -4.52 & $-4 \cdot 9$ & \\
\hline & -4.47 & -4.0 & 0.2 \\
\hline & -5.02 & -3.02 & -0 . \\
\hline & -4.72 & -2.9 & -1 \\
\hline & -5.86 & $-2 \cdot 0$ & \\
\hline & -6.20 & $-1 \cdot 2$ & -0 \\
\hline & -3 & -4 & -0 \\
\hline & -2.27 & -5 & 0 \\
\hline
\end{tabular}

\begin{tabular}{|c|c|c|c|}
\hline $\mathrm{H}$ & -2.251190 & -4.871654 & 1.485301 \\
\hline $\mathrm{C}$ & -1.156187 & -5.778595 & -0.100991 \\
\hline $\mathrm{H}$ & -0.280026 & -5.921338 & 0.523402 \\
\hline 0 & -1.103969 & -6.097519 & -1.460198 \\
\hline 0 & -2.255144 & -5.886848 & -2.225397 \\
\hline $\mathrm{H}$ & -2.262085 & -6.168805 & -3.274341 \\
\hline C & -3.382192 & -5.297630 & -1.670212 \\
\hline $\mathrm{H}$ & -4.265730 & -5.143859 & -2.28243 \\
\hline $\mathrm{C}$ & 0.208532 & -6.461887 & -2.047167 \\
\hline C & 1.130732 & -7.227071 & -1.33246 \\
\hline $\mathrm{H}$ & 0.814044 & -7.743457 & -0.431465 \\
\hline $\mathrm{C}$ & 2.466670 & -7.249174 & -1.69546 \\
\hline $\mathrm{H}$ & 3.165474 & -7.775839 & -1.058035 \\
\hline $\mathrm{C}$ & 2.943700 & -6.515930 & -2.785193 \\
\hline C & 1.985935 & -5.903338 & -3.600607 \\
\hline $\mathrm{H}$ & 2.290311 & -5.378647 & -4.499949 \\
\hline $\mathrm{C}$ & 0.648372 & -5.870782 & -3.234943 \\
\hline $\mathrm{H}$ & -0.046409 & -5.290291 & -3.833784 \\
\hline $\mathrm{C}$ & 4.393400 & -6.192722 & -2.878296 \\
\hline $\mathrm{C}$ & 5.372381 & -6.939933 & -2.210492 \\
\hline $\mathrm{H}$ & 5.13 & -7.932 & $-1 \cdot 8$ \\
\hline $\mathrm{C}$ & 6.626316 & -6.410107 & -1.941608 \\
\hline $\mathrm{H}$ & 7.331499 & -6.98 & -1.3515 \\
\hline $\mathrm{C}$ & 6.968057 & -5.121377 & -2.358668 \\
\hline $\mathrm{C}$ & 6.061964 & -4.457 & $-3.1 \varepsilon$ \\
\hline $\mathrm{H}$ & 6.297000 & -3.459037 & -3.5 \\
\hline $\mathrm{C}$ & 4.799308 & -4.973542 & -3.42 \\
\hline $\mathrm{H}$ & 4.086541 & $-4.352<$ & -3.9 \\
\hline $\mathrm{C}$ & 8.070355 & -4.340 & -1.74 \\
\hline $\mathrm{C}$ & 8.208568 & -4.366 & -0.3 \\
\hline $\mathrm{H}$ & 7.655121 & -5.095567 & 0.222443 \\
\hline $\mathrm{C}$ & 8.88 & -3.358 & 0.3 \\
\hline $\mathrm{H}$ & 8.828139 & -3.346994 & 1.3 \\
\hline $\mathrm{C}$ & 9.45 & -2.286 & -0.3 \\
\hline $\mathrm{C}$ & 9.484671 & -2.373557 & -1.776764 \\
\hline $\mathrm{H}$ & 9.994681 & -1.614448 & $-2 \cdot 36$ \\
\hline $\mathrm{C}$ & 8.793544 & -3.371342 & -2.448175 \\
\hline $\mathrm{H}$ & 8.770472 & -3.363366 & -3.53 \\
\hline $\mathrm{C}$ & 9.733902 & -1.012842 & 0.341038 \\
\hline $\mathrm{C}$ & 9.825006 & -0.951664 & 1.7 \\
\hline $\mathrm{H}$ & 10.016539 & -1.852605 & 2.311160 \\
\hline $\mathrm{C}$ & 9.584033 & 0.229699 & 2.422 \\
\hline $\mathrm{H}$ & 9.583926 & 0.221740 & 3.508616 \\
\hline $\mathrm{C}$ & 9.260070 & 1.404926 & 1.73 \\
\hline $\mathrm{C}$ & 9.384559 & 1.388202 & 0.347582 \\
\hline $\mathrm{H}$ & 9.138158 & 2.274258 & -0.227 \\
\hline C & 9.614871 & 0.204594 & -0.333468 \\
\hline $\mathrm{H}$ & 9.545229 & 0.225977 & -1.413763 \\
\hline $\mathrm{C}$ & 8.507096 & 2.517941 & 2.3655 \\
\hline $\mathrm{C}$ & 7.476920 & 2.200808 & 3.253 \\
\hline $\mathrm{H}$ & 7.396091 & 1.189302 & 3.638707 \\
\hline $\mathrm{C}$ & 6.453353 & 3.097300 & 3.515954 \\
\hline $\mathrm{H}$ & 5.599989 & 2.761138 & 4.095758 \\
\hline $\mathrm{C}$ & 6.424999 & 4.355553 & 2.910723 \\
\hline $\mathrm{C}$ & 7.553598 & 4.738582 & 2.176 \\
\hline $\mathrm{H}$ & 7.607017 & 5.738808 & 1.756818 \\
\hline $\mathrm{C}$ & 8.571195 & 3.835856 & 1.9027 \\
\hline
\end{tabular}




$\begin{array}{lrrr}\mathrm{H} & 9.396082 & 4.141894 & 1.266679 \\ \mathrm{C} & 5.131687 & 5.079701 & 2.818954 \\ \mathrm{C} & 4.143369 & 5.012679 & 3.805159 \\ \mathrm{H} & 4.393625 & 4.657749 & 4.800280 \\ \mathrm{C} & 2.827002 & 5.337797 & 3.511757 \\ \mathrm{H} & 2.073341 & 5.238865 & 4.286767 \\ \mathrm{C} & 2.444207 & 5.729836 & 2.223405 \\ \mathrm{C} & 3.455795 & 5.938677 & 1.285393 \\ \mathrm{H} & 3.207337 & 6.235444 & 0.270502 \\ \mathrm{C} & 4.769973 & 5.626064 & 1.586961 \\ \mathrm{H} & 5.507964 & 5.692708 & 0.801544 \\ \mathrm{C} & 1.026751 & 5.731064 & 1.790360 \\ \mathrm{C} & 0.167612 & 4.736531 & 2.265534 \\ \mathrm{H} & 0.521170 & 4.036339 & 3.015253 \\ \mathrm{C} & -1.550154 & 5.360355 & 0.674835 \\ \mathrm{C} & -0.714159 & 6.388507 & 0.241646 \\ \mathrm{H} & -1.048040 & 7.047049 & -0.546875 \\ \mathrm{C} & -1.090847 & 4.553143 & 1.719674 \\ \mathrm{H} & -1.687464 & 3.713998 & 2.060145 \\ \mathrm{C} & 0.547643 & 6.573678 & 0.784737 \\ \mathrm{H} & 1.173708 & 7.371193 & 0.395571 \\ \mathrm{C} & -3.005145 & 5.248743 & -1.378992 \\ \mathrm{H} & -2.243791 & 5.771550 & -1.947755 \\ \mathrm{C} & -3.844963 & 4.383276 & 0.677566 \\ \mathrm{H} & -3.769663 & 4.239666 & 1.747187 \\ \mathrm{C} & -4.937877 & 3.854524 & 0.017500 \\ \mathrm{H} & -5.658668 & 3.276652 & 0.586085 \\ \mathrm{C} & -4.104302 & 4.726900 & -2.046143 \\ \mathrm{H} & -4.187597 & 4.867521 & -3.119913 \\ \Delta \mathrm{H}=-5542.373912 & & \end{array}$

\section{$11 a$}

$\begin{array}{lrrr}\mathrm{C} & -8.854175 & -1.830600 & -0.471598 \\ \mathrm{C} & -8.625417 & -1.741299 & -1.843770 \\ \mathrm{H} & -8.414665 & -2.632837 & -2.424067 \\ \mathrm{C} & -8.784514 & 0.512294 & -1.738883 \\ \mathrm{C} & -9.246088 & -0.645508 & 0.150254 \\ \mathrm{H} & -9.542890 & -0.641193 & 1.193801 \\ \mathrm{C} & -8.275051 & 1.791957 & -2.270969 \\ \mathrm{C} & -7.399265 & 1.822385 & -3.355238 \\ \mathrm{H} & -7.331908 & 0.976727 & -4.031950 \\ \mathrm{C} & -6.600942 & 3.824681 & -2.669433 \\ \mathrm{C} & -8.389725 & 2.987969 & -1.566418 \\ \mathrm{H} & -9.129920 & 3.095673 & -0.780263 \\ \mathrm{C} & -5.394519 & 4.671793 & -2.562066 \\ \mathrm{C} & -4.278220 & 4.472529 & -3.375464 \\ \mathrm{H} & -4.355970 & 3.877702 & -4.279315 \\ \mathrm{C} & -2.985258 & 5.603869 & -1.905523 \\ \mathrm{C} & -5.210749 & 5.553493 & -1.499035 \\ \mathrm{H} & -6.042110 & 5.859140 & -0.874407 \\ \mathrm{C} & -1.629394 & 5.780289 & -1.345974 \\ \mathrm{C} & -0.469290 & 5.768426 & -2.117114 \\ \mathrm{H} & -0.525670 & 5.788891 & -3.200816 \\ \mathrm{C} & 0.801542 & 5.630718 & -0.244432 \\ \mathrm{C} & -1.436639 & 5.800694 & 0.031459\end{array}$

\begin{tabular}{|c|c|c|c|}
\hline $\mathrm{H}$ & -2.289563 & 5.832937 & 0.700963 \\
\hline $\mathrm{C}$ & 2.108416 & 5.311488 & 0.363959 \\
\hline & 3.198862 & 4.921813 & -0.405885 \\
\hline & 3.152065 & 4.982236 & -1.488 \\
\hline & 4.327433 & 4.309636 & 1.459549 \\
\hline 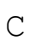 & 2.289335 & 5.253242 & 1.745527 \\
\hline & 1.508248 & 5.595906 & 2.4157 \\
\hline & 5.422423 & 3.490683 & 2.016813 \\
\hline & 6.615459 & 3.281552 & 1.3301 \\
\hline & 6.885183 & 3.898719 & 0.479521 \\
\hline & 7.075165 & 1.491317 & 2.6355 \\
\hline & 5.259809 & 2.734456 & 3.1755 \\
\hline & 4.419180 & 2.915026 & 3.838406 \\
\hline & 7.693578 & 0.150584 & 2.674360 \\
\hline & 8.626452 & -0.262741 & 1.721295 \\
\hline & 9.150652 & 0.460867 & 1.108499 \\
\hline & 8.139420 & -2.424547 & 2.173239 \\
\hline & 7.204034 & -0.856723 & 3.507003 \\
\hline $\mathrm{H}$ & 6.570457 & -0.612726 & 4.353105 \\
\hline & 7.946832 & -3.768552 & \\
\hline & 8.224004 & -4.014423 & 0.249964 \\
\hline $\mathrm{H}$ & 8.837767 & -3.322488 & -0.317738 \\
\hline 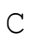 & 6.891904 & -5.843755 & 0.289264 \\
\hline & 7.229978 & -4.765372 & 2.2521 \\
\hline $\mathrm{H}$ & 7.034092 & -4.692239 & 3.316688 \\
\hline c & 6.012434 & -6.752640 & -0.47 \\
\hline & 5.820718 & -6.599181 & -1.843770 \\
\hline 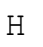 & 6.487436 & -5.970895 & -2.424067 \\
\hline$C$ & 3.948597 & -7.863759 & -1.738883 \\
\hline C & 5.182070 & -7.684593 & 0.1502 \\
\hline $\mathrm{H}$ & 5.326734 & 3789 & 1.193801 \\
\hline c & 2.585645 & -8.062383 & -2.270969 \\
\hline$C$ & 2.121401 & -7.319144 & -3.355238 \\
\hline $\mathrm{H}$ & 2.820084 & -6.837982 & -4.03195 \\
\hline $\mathrm{C}$ & -0.011800 & -7.628924 & -2.669433 \\
\hline C & 1.607205 & -8.759699 & -1.566418 \\
\hline $\mathrm{H}$ & 1.884029 & -9.454579 & -0.780263 \\
\hline $\mathrm{C}$ & -1.348632 & -7.007687 & -2.562066 \\
\hline C & -1.734214 & -5.941312 & -3.37546 \\
\hline $\mathrm{H}$ & -1.180203 & -5.711232 & -4.27931 \\
\hline$C$ & -3.360464 & -5.387244 & -1.90552 \\
\hline C & -2.204092 & -7.289388 & -1.499035 \\
\hline $\mathrm{H}$ & -2.053109 & -8.162191 & -0.874407 \\
\hline C & -4.191180 & -4.301241 & -1.345 \\
\hline C & -5.277116 & -2.121203 & -0.2444 \\
\hline C & -8.506792 & -3.046686 & 0.28926 \\
\hline C & -7.741922 & -3.878659 & 2.25215 \\
\hline $\mathrm{H}$ & -7.580644 & -3.745583 & 3.31668 \\
\hline C & -7.237078 & -4.997882 & 1.593308 \\
\hline C & -7.588594 & -5.114985 & 0.24996 \\
\hline $\mathrm{H}$ & -7.296242 & -5.992487 & -0.31773 \\
\hline $\mathrm{C}$ & -6.169429 & -5.836671 & 2.17323 \\
\hline C & -4.343961 & -5.810515 & 3.5070 \\
\hline $\mathrm{H}$ & -3.815865 & -5.383820 & 4.3531 \\
\hline C & 6379 & -6.738126 & 2.674360 \\
\hline C & -4.540766 & -7.339356 & 1.72129 \\
\hline & -4.176203 & -8.155131 & 1.1084 \\
\hline
\end{tabular}




\begin{tabular}{|c|c|c|c|}
\hline & -2.246064 & -6.872931 & - \\
\hline & -0.261796 & -5.922356 & \\
\hline & 0.314897 & -5.284635 & \\
\hline & 0.311809 & -6.441298 & \\
\hline & -0.465822 & -7.369932 & \\
\hline & -0.06 & -7.912103 & \\
\hline & 1.568538 & -5.902485 & \\
\hline & & -4.60 & \\
\hline & 4.092 & -4.10 & \\
\hline & & -4.48 & \\
\hline & 2.66 & -5.23 & \\
\hline & & -5.2 & \\
\hline & 4.47 & -3.50 & \\
\hline & 5.74 & $-1 \cdot 6$ & \\
\hline & 6.19 & -0.93 & \\
\hline & & $-1 \cdot 4$ & -1.3 \\
\hline & 5.23 & -2.47 & -2.1 \\
\hline & & -2 & \\
\hline & 6.34 & -0.2 & \\
\hline & 7.41 & 1.7 & -1 \\
\hline & 8 . & & \\
\hline & 6.74 & 2 . & -2.5 \\
\hline & 6.01 & & -3 \\
\hline & 5.53 & & -4.2 \\
\hline & 6. & & \\
\hline & 6.78 & 5.7 & -1 \\
\hline & & & -0 \\
\hline & 5.68 & 6.27 & -2.2 \\
\hline & 5 . & 5 . & -3 \\
\hline & 4.51 & 5 . & -4 \\
\hline & & & -1 \\
\hline & 4.06 & 8.33 & 0 \\
\hline & 4.21 & 8.58 & \\
\hline & 2.84 & 8 . & -0 . \\
\hline & 2.80 & 8.3 & -1 \\
\hline & 1.92 & 8.60 & -2 . \\
\hline & 1.61 & 8 . & \\
\hline & 0.51 & 8.6 & 2 \\
\hline & 0.54 & 8. & \\
\hline & -0 & 8.76 & 1 \\
\hline & -0 & 9.12 & \\
\hline & -1.54 & 9. & -0.3 \\
\hline & -1 & 8.2 & \\
\hline & -2 . & 6. & \\
\hline & -2 . & 5 . & \\
\hline & -3.97 & 6.5 & 2. \\
\hline & -4 & 7. & \\
\hline & -4 & 7.6 & 1 . \\
\hline $\mathrm{C}$ & -4 & 5 . & \\
\hline & -4.99 & 3. & \\
\hline & -4 & 2 . & \\
\hline & -5 & 2 . & \\
\hline C & -6 & 4. & \\
\hline & -6 & & \\
\hline 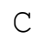 & -5 & 1 . & \\
\hline & -5 & -0 . & \\
\hline & -5 . & 0.3 & \\
\hline
\end{tabular}

\begin{tabular}{|c|c|c|c|}
\hline & -5.890775 & 38650 & -1.488665 \\
\hline & -5.694109 & -0.643999 & 1.745527 \\
\hline & -5.600321 & -1.491772 & 2.41 \\
\hline & -4.305229 & -4.144513 & 0.031459 \\
\hline & -3.906690 & -4.899288 & 0.70096 \\
\hline & -4.760958 & -3.290630 & -2.1171 \\
\hline & -4.750492 & -3.349689 & -3.200816 \\
\hline & -3.076362 & 4.940292 & -3 \\
\hline & -4.010793 & 6.008395 & \\
\hline & 0.740721 & 5.702854 & -1.5766 \\
\hline & -0.235513 & 5.724171 & 0.5877 \\
\hline & -6.557278 & 2.828377 & -3.5549 \\
\hline & -7.565678 & 4.008001 & -1.765 \\
\hline V & 4.307606 & 4.2 & \\
\hline N & 3.387320 & 4.751842 & 2.297087 \\
\hline & 7.449044 & 2.298086 & 1.641063 \\
\hline & 6.070036 & 1.727628 & 3.4815 \\
\hline IV & 8.853895 & -1.545674 & 1.4689 \\
\hline & 7.420600 & -2.142190 & \\
\hline & 7.6 & -5.040605 & -0 . \\
\hline N & 6.706627 & -5.801880 & 1.611505 \\
\hline N & 4.794016 & -7.147477 & -2.48092 \\
\hline$N$ & 4.154977 & -8.244454 & -0.47527 \\
\hline N & 0.829193 & -7.092958 & -3.55494 \\
\hline $\mathrm{N}$ & 0.3 & -8.5 & -1 \\
\hline$N$ & -2.740237 & -5.134354 & -3.059896 \\
\hline $\mathrm{N}$ & -3.198026 & -6.477646 & -1.157411 \\
\hline N & -5.309177 & -2.209944 & -1.576671 \\
\hline $\mathrm{N}$ & -4.839521 & -3.066046 & 0.58774 \\
\hline N & -5.987256 & 1.517251 & 0.128904 \\
\hline N & -5.808876 & 0.5 & 2 . \\
\hline N & -5.714723 & 5.302018 & 1.641063 \\
\hline N & -4.531188 & 4.392991 & 3.481542 \\
\hline $\mathrm{N}$ & -3.088355 & 8.440535 & 1.468971 \\
\hline N & -1.855109 & 7.497523 & 3.26236 \\
\hline N & 0.516261 & 9.187019 & -0.40783 \\
\hline N & 1.6 & 8.7 & 05 \\
\hline $\mathrm{N}$ & 3.792889 & 7.7 & -2 . \\
\hline $\mathrm{N}$ & 5.062418 & 7.720543 & -0.475273 \\
\hline $\mathrm{N}$ & 5.728085 & 4.264581 & -3.554945 \\
\hline $\mathrm{N}$ & 7.253870 & 4.548069 & -1.765491 \\
\hline N & 7.208819 & 0.469251 & -1.157411 \\
\hline N & 5.816 & 0.194062 & -3 \\
\hline 1v & 5.075034 & -2.658125 & 0.587742 \\
\hline $\mathrm{N}$ & 4.568456 & -3.492910 & -1.576671 \\
\hline $\mathrm{N}$ & 2.421556 & -5.309426 & 2.297087 \\
\hline $\mathrm{N}$ & 1.679650 & -5.943741 & 0.128904 \\
\hline $\mathrm{N}$ & -1.538848 & -6.120619 & 3.481542 \\
\hline $\mathrm{N}$ & -1.734321 & -7.600104 & 1.641063 \\
\hline N & -5.565491 & -5.355333 & 3.262362 \\
\hline $\mathrm{N}$ & -5.765540 & -6.894861 & 1.468971 \\
\hline $\mathrm{N}$ & -8.377889 & -2.907169 & 1.61150 \\
\hline $\mathrm{N}$ & -8.214322 & -4.146414 & -0.407837 \\
\hline $\mathrm{N}$ & -9.217395 & 0.523911 & -0.47 \\
\hline N & 8690 & -0.578001 & -2.480927 \\
\hline & & & \\
\hline
\end{tabular}




\section{$11 b$}

\begin{tabular}{|c|c|c|c|}
\hline & 1.817518 & 85 & -0 \\
\hline & 3.778066 & 7.881703 & -1.899873 \\
\hline & 3.117762 & 8.821419 & 0 \\
\hline & 635 & 9.1 & \\
\hline & 69816 & 6.877567 & \\
\hline & 07 & 4. & \\
\hline & 1 & 6.570778 & \\
\hline & & & \\
\hline & & & \\
\hline & & & \\
\hline & & & -1 \\
\hline & 7.47 & & -1 \\
\hline & & -0.81 & \\
\hline & & -2.9 & \\
\hline & 6.08 & -1.04 & -0 \\
\hline & & -0.3 & \\
\hline & 4.12 & -4.02 & \\
\hline & & -5 & \\
\hline & 3. & -4 & \\
\hline & & -3.4 & \\
\hline & & -6 . & \\
\hline & -1 & -6 . & \\
\hline & & -5 & \\
\hline & 5 & -5 & \\
\hline & & -6 . & \\
\hline & -5 & -6.2 & \\
\hline & & -5 & \\
\hline & -3 & -5.22 & \\
\hline & & -5 . & \\
\hline & -8.20 & -3 & \\
\hline & -7 & -4 & \\
\hline & -7 & -4.26 & \\
\hline 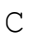 & -8 & -2.8 & -0 \\
\hline & -8 & -0.66 & -1 \\
\hline & -9 & -1 & \\
\hline & -9 & $-1 \cdot 6$ & 1 . \\
\hline C & -8 & 0.6 & -2 \\
\hline & -6 . & 2 . & -2 \\
\hline C & -8 & 1.8 & -1 \\
\hline & -9.50 & 1.93 & -1 \\
\hline $\mathrm{C}$ & -5.75 & 3.75 & -2 . \\
\hline 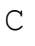 & -3 & 5.11 & -2 \\
\hline C & -5 & 4. & -1 \\
\hline & -6.6 & 4.75 & -1.1 \\
\hline $\mathrm{C}$ & -2.2 & 5.5 & -1 \\
\hline & 0.12 & 5.7 & -0 . \\
\hline $\mathrm{C}$ & 0. & 9. & \\
\hline 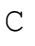 & -0.24 & 8 . & \\
\hline $\mathrm{H}$ & -0 & 8 . & \\
\hline 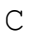 & -1.5 & & \\
\hline $\mathrm{C}$ & -2 & 7 . & 2. \\
\hline $\mathrm{C}$ & -3 & & \\
\hline$\Pi$ & -2 . & & 4. \\
\hline & -4 & & \\
\hline & -5 . & & \\
\hline
\end{tabular}

\begin{tabular}{|c|c|c|c|}
\hline $\mathrm{C}$ & -5.154824 & 2.408428 & 3.294895 \\
\hline . & -4.875141 & 1.589545 & 3.947564 \\
\hline C & -5.834154 & 2.166011 & 2.096560 \\
\hline C & -5.922304 & 0.803072 & 1.526742 \\
\hline C & -5.487041 & -1.416860 & 1.726418 \\
\hline & -5.259009 & -2.268612 & 2.35670 \\
\hline C & -5.548158 & -1.556070 & 0.334735 \\
\hline C & -5.051279 & -2.774392 & -0.34033 \\
\hline C & -3.943837 & -4.747234 & -0.162 \\
\hline $\mathrm{H}$ & -3.572187 & -5.555880 & 0.45410 \\
\hline C & -3.667371 & -4.722068 & -1.53106 \\
\hline C & -2.649631 & -5.633609 & -2.09971 \\
\hline C & -1.168405 & -7.315328 & -1.73160 \\
\hline $\mathrm{H}$ & -0.834298 & -8.151043 & -1.129037 \\
\hline 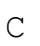 & -0.407054 & -6.885856 & -2.82 \\
\hline C & 1.001667 & -7.316553 & -2.956766 \\
\hline C & 2.730095 & -8.412893 & -1.96 \\
\hline $\mathrm{H}$ & 3.072845 & -9.196743 & -1.299493 \\
\hline C & 3.621240 & -7.482963 & -2.51 \\
\hline $\mathrm{C}$ & 4.936722 & -7.212753 & -1.8998 \\
\hline $\mathrm{C}$ & 6.080692 & -7.110770 & 0.06 \\
\hline $\mathrm{H}$ & 6.216927 & -7.462943 & 1.07 \\
\hline $\mathrm{C}$ & 6.829252 & -6.041559 & -0.4 \\
\hline C & 7.552131 & -5.109126 & \\
\hline $\mathrm{C}$ & 7.577368 & -4.089182 & 2.47 \\
\hline $\mathrm{H}$ & 7.291211 & -4.092956 & 85 \\
\hline $\mathrm{C}$ & 8.213849 & -2.983594 & 1.903932 \\
\hline $\mathrm{C}$ & 8.146831 & -1.641868 & 91 \\
\hline $\mathrm{C}$ & 6.841266 & -0.216705 & 3.713913 \\
\hline $\mathrm{H}$ & 6.075624 & -0.065794 & 4.4 \\
\hline $\mathrm{C}$ & 7.332309 & 0.853790 & 2.957937 \\
\hline $\mathrm{C}$ & 6.564293 & 2.110675 & \\
\hline $\mathrm{C}$ & 4.663172 & 3.259995 & 3.294895 \\
\hline $\mathrm{H}$ & 3.814157 & 3.427224 & 564 \\
\hline $\mathrm{C}$ & 4.792898 & 3.969520 & 2.096560 \\
\hline $\mathrm{C}$ & 3.656633 & 4.727330 & 1.526 \\
\hline $\mathrm{C}$ & 1.426483 & 5.582880 & 0.334735 \\
\hline $\mathrm{C}$ & 1.516484 & 5.460347 & 1.726418 \\
\hline $\mathrm{H}$ & 0.664828 & 5.688741 & 2.356700 \\
\hline $\mathrm{C}$ & -2.139307 & 5.789080 & -0.162540 \\
\hline $\mathrm{H}$ & -3.025440 & 5.871545 & 0.454108 \\
\hline $\mathrm{N}$ & 6.903564 & 1.378428 & -1.352136 \\
\hline $\mathrm{N}$ & 5.563222 & -2.104237 & 0.430887 \\
\hline $\mathrm{N}$ & 6.474654 & 5.385306 & -2.160860 \\
\hline $\mathrm{N}$ & 3.027263 & -4.766460 & 2.312284 \\
\hline $\mathrm{N}$ & -0.761491 & -5.938440 & 3.632 \\
\hline $\mathrm{N}$ & -4.875607 & -5.527654 & 3.4617 \\
\hline $\mathrm{N}$ & -8.05 & -3.692250 & 1.739855 \\
\hline $\mathrm{N}$ & -9.204506 & -0.601583 & -0.661448 \\
\hline $\mathrm{N}$ & -7.901139 & 2.914562 & -2.160860 \\
\hline $\mathrm{N}$ & -4.645536 & 5.289448 & -1.352136 \\
\hline $\mathrm{N}$ & -0.959289 & 5.870010 & 0.430887 \\
\hline $\mathrm{N}$ & 2.614244 & 5.004917 & 2.312284 \\
\hline $\mathrm{N}$ & 5.523585 & 2.309749 & 3.632634 \\
\hline $\mathrm{N}$ & 7.224893 & -1.458572 & 3.46170 \\
\hline $\mathrm{N}$ & 7.224893 & -5.129381 & 1.73985 \\
\hline $\mathrm{N}$ & 5.123239 & -7.670545 & -0.66144 \\
\hline
\end{tabular}




\begin{tabular}{|c|c|c|c|}
\hline & 26485 & -8.299868 & -2 \\
\hline & -2.258028 & -6.667876 & -1.352136 \\
\hline & -4.603934 & -3.765773 & 0887 \\
\hline & -5.641507 & -0.238457 & 2 \\
\hline & -4.762094 & 3.628691 & \\
\hline & -2.34 & & \\
\hline & 0.829728 & 8.821 & \\
\hline & 4.08 & & \\
\hline & 8.8 & -0.547 & \\
\hline & -4.88 & -7.3 & \\
\hline & -3.93 & 7.917 & 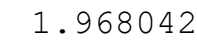 \\
\hline & & & \\
\hline & -0.83 & -7.35 & \\
\hline & -5 & & \\
\hline & 3.61 & 4.99 & \\
\hline & 2.52 & -5.62 & \\
\hline & -6.13 & 0.62 & \\
\hline & & & -1 \\
\hline & 4.90 & -2.83 & \\
\hline & -4.90 & -2.83 & -1 \\
\hline & -3 & & \\
\hline & 5.61 & 0 . & -3 \\
\hline & -1 & -5.3 & \\
\hline & -6 & 1.627 & -3 \\
\hline & 4.73 & & \\
\hline & 1.91 & -6.57 & -3.7 \\
\hline & -8 & -1 & -2 \\
\hline & 5.84 & -6.320 & -2.4 \\
\hline C & 2 . & 8.2 & -2 \\
\hline 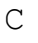 & -7.76 & -5.21 & -0 . \\
\hline C & 8 . & -4.1 & -0 \\
\hline C & -0.63 & 9.33 & -0 . \\
\hline$v$ & -3.60 & -7.610790 & \\
\hline & -4.79 & 6.923 & 2. \\
\hline & 8 & 0.68 & \\
\hline & 0.38 & -7.02 & 1 . \\
\hline V & -6.27 & 3.17 & \\
\hline & 5. & 3. & 1 . \\
\hline 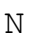 & 3.4 & -4 & -0 . \\
\hline & -5.92 & -0.53 & -0 . \\
\hline $\mathrm{N}$ & 2.45 & 5.3 & -0 \\
\hline & 5.37 & -1.74 & -2 \\
\hline N & -4.15 & $-3.7 \varepsilon$ & -2 . \\
\hline N & -1.17 & 5.52 & -2 \\
\hline $\mathrm{N}$ & 5 & 2 . & -3 \\
\hline $\mathrm{N}$ & -4.70 & 3.70 & -3 \\
\hline $\mathrm{N}$ & -0.8 & -5.9 & -3 \\
\hline $\mathrm{N}$ & 4.14 & 6.10 & -3 \\
\hline $\mathrm{N}$ & -7 & 0 . & -3 \\
\hline $\mathrm{N}$ & 3.21469 & -6.63 & -3 \\
\hline $\mathrm{N}$ & 1 & 8 . & -1 \\
\hline . & -8 & -3.00 & -1 \\
\hline $\mathrm{N}$ & 6. & -5.7 & -1 \\
\hline & -1 & 9. & \\
\hline $\mathrm{N}$ & -6 & -6 & \\
\hline & & -3 & \\
\hline & 5. & 0 . & -3 \\
\hline
\end{tabular}

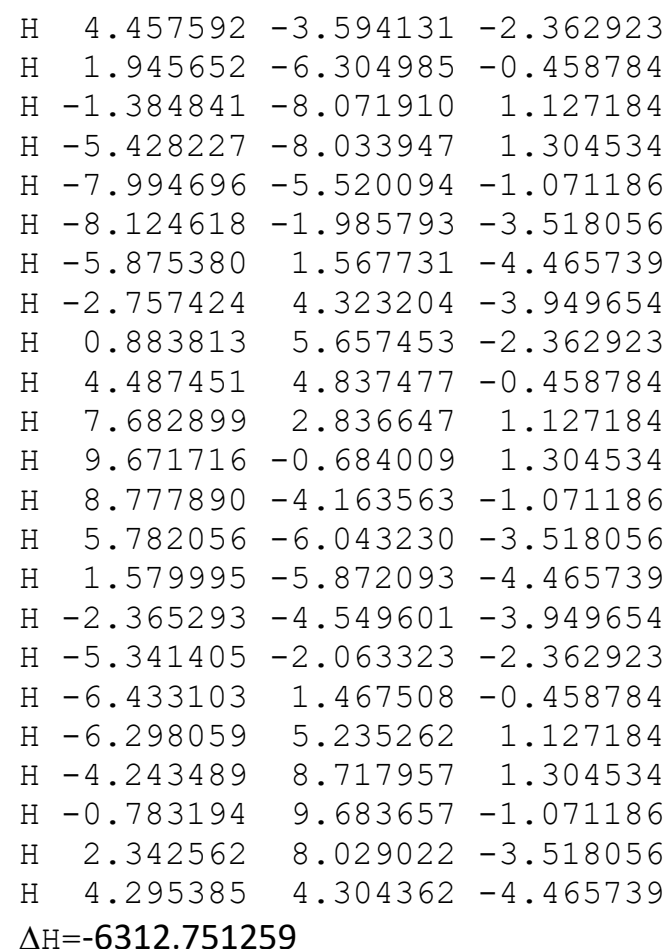

11c

C $-1.033438-8.858694-0.415435$

C $-0.963137-8.675905-1.800838$

$\mathrm{H}-0.012995-8.765660-2.310486$

C $-3.252877-8.104495-1.653333$

C $-4.397018-7.275407-2.113693$

C $-5.858608 \quad-5.115794-2.583212$

C $-5.567016-7.121681-1.363281$

$\mathrm{H}-5.790872-7.805635-0.555340$

C $-6.247542-3.681036-2.598525$

C $-5.706285-2.774790-3.518908$

$\mathrm{H}-5.208287-3.136113-4.409242$

C $-6.307392-1.114482-1.945349$

C $-6.032742 \quad 0.188908-1.294741$

C $-5.078384 \quad 2.424192-0.236960$

$\begin{array}{llll}\text { C } & -5.948417 & 0.259554 & 0.098799\end{array}$

$\mathrm{H}-6.210720-0.602518 \quad 0.695690$

$\begin{array}{llll}\text { C }-4.319719 & 3.606416 & 0.239333\end{array}$

C $-3.5663394 .378398-0.648869$

$\mathrm{H}-3.632838 \quad 4.209509-1.716197$

C $-2.684308 \quad 5.405358 \quad 1.295586$

C $-1.613400 \quad 6.198021 \quad 1.945616$

$\begin{array}{llll}\text { C } & 0.744827 & 7.213168 & 2.598122\end{array}$

C $-1.081514 \quad 5.827187 \quad 3.185292$

$\mathrm{H}-1.600149 \quad 5.103318 \quad 3.801630$

$\begin{array}{llll}\text { C } & 2.211199 & 7.457164 & 2.582624\end{array}$

$\begin{array}{llll}\text { C } & 2.821477 & 8.249188 & 1.603072\end{array}$

H $2.225418 \quad 8.913139 \quad 0.991945$

$\begin{array}{llll}\text { C } \quad 4.783130 \quad 7.027117 & 2.113017\end{array}$

C $\quad 6.013254 \quad 6.331953 \quad 1.652701$ 


\begin{tabular}{|c|c|c|c|}
\hline & 7.623336 & 4.628651 & 0.414470 \\
\hline & 6.655132 & 5.371904 & 2.441343 \\
\hline & 6.424402 & 5.287587 & 3.494990 \\
\hline & 8.188573 & 3.534364 & -0.415435 \\
\hline & 7.995122 & 3.503852 & -1.800838 \\
\hline & 7.597782 & 4.371576 & -2.310486 \\
\hline & 8.645137 & 1.235173 & -1.653333 \\
\hline & 8.499196 & -0.170226 & -2.113693 \\
\hline & 7.359711 & -2.515806 & -2.583212 \\
\hline & 8.951065 & -1.260337 & -1.363281 \\
\hline & 9.655314 & -1.112225 & -0.555340 \\
\hline & 6.311642 & -3.570013 & -2.598525 \\
\hline & 5.256181 & -3.554392 & -3.518908 \\
\hline & 5.320097 & -2.942453 & -4.409242 \\
\hline & 4.118865 & -4.905121 & -1.945349 \\
\hline & 2.852771 & -5.318962 & -1.294741 \\
\hline & 0.439780 & -5.610106 & -0.236960 \\
\hline & 0.196861 & -8.916328 & 0.414470 \\
\hline & 1.324639 & -8.449465 & 2.441343 \\
\hline & 1.366984 & -8.207489 & 3.494990 \\
\hline & 2.477006 & -8.373607 & 1.652701 \\
\hline & 3.694097 & -7.655871 & 2.113017 \\
\hline & 5.352494 & -5.643536 & 2.582624 \\
\hline & 5.733268 & -6.568064 & 1.603072 \\
\hline & 6.606296 & -6.383838 & 0.991945 \\
\hline & 5.874374 & -4.251623 & 2.598122 \\
\hline & 5.587249 & -1.976975 & 3.185292 \\
\hline & 5.219678 & -1.165889 & 3.801630 \\
\hline & 6.174343 & -1.701765 & 1.945616 \\
\hline & 6.023332 & -0.378000 & 1.295586 \\
\hline & 5.283108 & 1.937778 & 9333 \\
\hline & 5.574973 & 0.899341 & -0.648869 \\
\hline & 5.461961 & 1.041375 & -1.716197 \\
\hline & 4.638604 & 3.185914 & -0.236960 \\
\hline & 3.198989 & 5.021703 & 0.098799 \\
\hline & 2.583564 & 5.679900 & 0.695690 \\
\hline & 3.179970 & 5.130053 & -1.294741 \\
\hline & 2.188526 & 6.019602 & -1.945349 \\
\hline & -0.064099 & 7.251048 & -2.598525 \\
\hline & 0.450104 & 6.329182 & -3.518908 \\
\hline & -0.111810 & 6.078565 & -4.409242 \\
\hline & -1.501104 & 7.631600 & -2.583212 \\
\hline 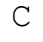 & -3.384049 & 8.382018 & -1.363281 \\
\hline I & -3.864442 & 8.917859 & -0.555340 \\
\hline & -4.102178 & 7.445633 & -2.113693 \\
\hline & -5.392260 & 6.869321 & -1.653333 \\
\hline & -7.155135 & 5.324330 & -0.415435 \\
\hline & -7.031985 & 5.172053 & -1.800838 \\
\hline I & -7.584787 & 4.394084 & -2.310486 \\
\hline & -7.820197 & 4.287678 & 0.414470 \\
\hline & -7.979771 & 3.077561 & 2.441343 \\
\hline & -7.791386 & 2.919901 & 3.494990 \\
\hline & -8.490259 & 2.041654 & 1.652701 \\
\hline & -8.477227 & 0.628753 & 2.113017 \\
\hline & -7.563692 & -1.813628 & 2.582624 \\
\hline & -8.554745 & -1.681123 & 1.603072 \\
\hline & -8.83171 & -2.529301 & 0.991945 \\
\hline
\end{tabular}




$\begin{array}{lrrr}\mathrm{C} & -2.034333 & 8.478082 & -1.604209 \\ \mathrm{~N} & -1.140802 & -5.613102 & 1.557632 \\ \mathrm{~N} & -4.290688 & 3.794515 & 1.557632 \\ \mathrm{~N} & 5.431490 & 1.818587 & 1.557632 \\ \mathrm{~N} & 1.807747 & -5.553655 & -2.083383 \\ \mathrm{~N} & -5.713480 & 1.211272 & -2.083383 \\ \mathrm{~N} & 3.905733 & 4.342383 & -2.083383 \\ \mathrm{~N} & 6.313609 & -4.377696 & -1.541403 \\ \mathrm{~N} & -6.948001 & -3.278898 & -1.541403 \\ \mathrm{~N} & 0.634391 & 7.656594 & -1.541403 \\ \mathrm{~N} & 7.686669 & -0.319847 & -3.160886 \\ \mathrm{~N} & -4.120330 & -6.496927 & -3.160886 \\ \mathrm{~N} & -3.566339 & 6.816774 & -3.160886 \\ \mathrm{~N} & 8.730403 & 2.514590 & 0.249759 \\ \mathrm{~N} & -2.187503 & -8.818046 & 0.249759 \\ \mathrm{~N} & -6.542900 & 6.303456 & 0.249759 \\ \mathrm{~N} & 6.304727 & 6.505590 & 0.364291 \\ \mathrm{~N} & 2.481643 & -8.712849 & 0.364291 \\ \mathrm{~N} & -8.786370 & 2.207259 & 0.364291 \\ \mathrm{~N} & 2.913520 & 6.665346 & 3.393218 \\ \mathrm{~N} & 4.315600 & -5.855855 & 3.393218 \\ \mathrm{~N} & -7.229119 & -0.809491 & 3.393218 \\ \mathrm{~N} & -1.077589 & 7.168703 & 1.208234 \\ \mathrm{~N} & 6.747074 & -2.651132 & 1.208234 \\ \mathrm{~N} & -5.669484 & -4.517571 & 1.208234 \\ \mathrm{H} & 0.683417 & 6.041383 & 4.408646 \\ \mathrm{H} & 4.684793 & 8.513873 & 0.553616 \\ \mathrm{H} & 7.962671 & 3.660577 & 2.308683 \\ \mathrm{H} & 8.033504 & 2.175504 & -3.496071 \\ \mathrm{H} & 8.556123 & -3.347897 & -0.993584 \\ \mathrm{H} & 3.233469 & -4.261191 & -3.801193 \\ \mathrm{H} & 1.330539 & -5.396562 & 1.717947 \\ \mathrm{H} & -4.086273 & -4.716419 & -0.695843 \\ \mathrm{H} & -5.573700 & -2.428835 & 4.408646 \\ \mathrm{H} & -9.715627 & -0.199787 & 0.553616 \\ \mathrm{H} & -7.151488 & 5.065587 & 2.308683 \\ \mathrm{H} & -5.900794 & 5.869467 & -3.496071 \\ \mathrm{H} & -1.378698 & 9.083768 & -0.993584 \\ \mathrm{H} & 2.073565 & 4.930862 & -3.801193 \\ \mathrm{H} & 4.008290 & 3.850562 & 1.717947 \\ \mathrm{H} & 6.127675 & -1.180607 & -0.695843 \\ \mathrm{H} & 4.890283 & -3.612549 & 4.408646 \\ \mathrm{H} & 5.030834 & -8.314086 & 0.553616 \\ \mathrm{H} & -0.811183 & -8.726164 & 2.308683 \\ \mathrm{H} & -2.132710 & -8.044971 & -3.496071 \\ \mathrm{H} & -7.177425 & -5.735872 & -0.993584 \\ \mathrm{H} & -5.307034 & -0.669671 & -3.801193 \\ \mathrm{H} & -5.338830 & 1.546000 & 1.717947 \\ \mathrm{H} & -2.041402 & 5.897026 & -0.695843 \\ \Delta \mathrm{H}=-6312.064743 & & \\ & & \end{array}$

